\title{
WestVirginiaUniversity
}

THE RESEARCH REPOSITORY @ WVU

Graduate Theses, Dissertations, and Problem Reports

2001

\section{Toward high-definition reservoir characterization}

Gheorghe Luca

West Virginia University

Follow this and additional works at: https://researchrepository.wvu.edu/etd

\section{Recommended Citation}

Luca, Gheorghe, "Toward high-definition reservoir characterization" (2001). Graduate Theses,

Dissertations, and Problem Reports. 1200.

https://researchrepository.wvu.edu/etd/1200

This Thesis is protected by copyright and/or related rights. It has been brought to you by the The Research Repository @ WVU with permission from the rights-holder(s). You are free to use this Thesis in any way that is permitted by the copyright and related rights legislation that applies to your use. For other uses you must obtain permission from the rights-holder(s) directly, unless additional rights are indicated by a Creative Commons license in the record and/ or on the work itself. This Thesis has been accepted for inclusion in WVU Graduate Theses, Dissertations, and Problem Reports collection by an authorized administrator of The Research Repository @ WVU. For more information, please contact researchrepository@mail.wvu.edu. 


\title{
TOWARD HIGH DEFINITION RESERVOIR CHARACTERIZATION
}

\author{
Gheorghe Luca
}

\begin{abstract}
Thesis submitted to the
College Of Engineering And Mineral

at West Virginia University

in partial fulfillment of the requirements for the degree of

Master Of Science In Petroleum And Natural Gas Engineering
\end{abstract}

\author{
Sam Ameri, Chair \\ Thomas Wilson, Ph. D \\ Frances C. Toro, Ph. D \\ Shahab Mohaghegh, Ph. D \\ Department Of Petroleum And Natural Gas Engineering \\ Morgantown, West Virginia \\ 2001
}

Keywords: Seismic, Crosswe ll seismic, Neural Networks, Resolution 


\title{
ABSTRACT \\ TOWARD HIGH DEFINITION RESERVOIR CHARACTERIZATION
}

\author{
Gheorghe Luca
}

In this project a new methodology for high resolution reservoir characterization is proposed. Surface seismic experiments suffer from the low resolution but it has an enormous advantage-its coverage. Crosswell seismic experiments have a better resolution but the zone covered is small in magnitude comparing to the field size.

The process involves deconvolution of a low resolution signal (surface seismic traces) into a high resolution crosswell seismic trace. The problem has multiple solutions and an analytical solution is hard or difficult to be developed.

This project proved that it is possible to predict high- resolution crosswell seismic traces using low- definition surface seismic traces. The statistical comparison between the real and the synthetic crosswell seismic traces gives confidence that future work in this area worth the effort.

The ultimate goal of this work is to be a part of an intelligent tool that will be able to provide, based on surface seismic, a volumetric, detailed picture of rock and fluid characteristics across the field. 


\section{ACKNOWLEDGMENTS}

First I would like to express my sincere thanks and deepest gratitude to my advisor, Dr. Shahab Mohaghegh for his support and guidance throughout the project, as well as for the financial support provided.

It was also a pleasure to learn and share information with Dr. Shahab Mohaghegh, with Andrei Popa and Razi Gaskari during our meetings.

There are many people who helped me over the last years.

To Dr. Dan Vladimir Nichita for his continuous encouragement and advices.

I would like to thank to my former supervisor, Dr. Stefan Traian Mocuta, Member of Parliament. He accepted to review my projects during my employment period and helped me to improve them.

During my BS, I was honored to have Dr. Ion Cretu as advisor, at Ploiesti Oil and Gas University in Romania. I really appreciate his support then and many years later.

I am thankful to Dr. and Mrs. Sinisha Jikich; they accommodated me with the differences across continents and gave me support at every time.

I am also grateful to Mr. Viorel Voinescu to give me the recommendations and advices to start my master's program in WVU.

To Andrei Popa, a real friend, thanks for your help. I wish him the best during his Ph. D Dissertation.

I would like to take this opportunity to thank the entire staff of the Petroleum and Natural Gas Engineering Department for their cooperation and encouragement. Thanks for the Committee members for their advices.

I would like to extend my gratitude and deepest appreciation to my parents, Ion and Ana Luca and the rest of my family; unfortunately they had to learn that there is nothing possible without sacrifice and I am thankful that they forgave that. To my wife, Denis, best wishes.

To West Virginia University and to USA. I found a home here, in Morgantown.

For all the abovementioned and many other individuals, God bless you, wherever you are, guide your steps and let your dreams become reality!

I also thank to my former managers for their encouragements; they made me to understand that this Master Degree will be useful for me in the future and helped me find the right way. 


\section{TABLE OF CONTENTS}

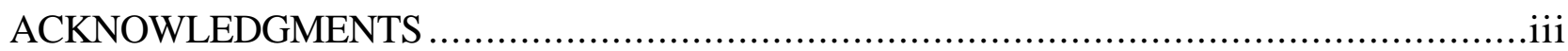

TABLE OF CONTENTS ......................................................................... iv

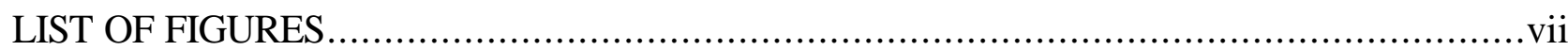

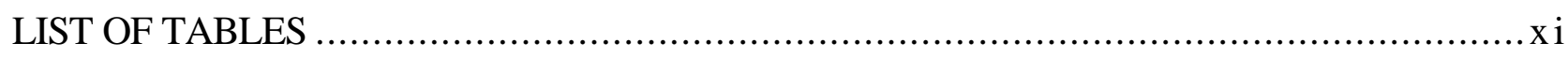

EXECUTIVE SUMMARY ..................................................................... 1

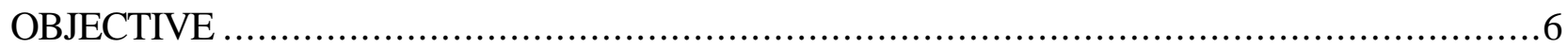

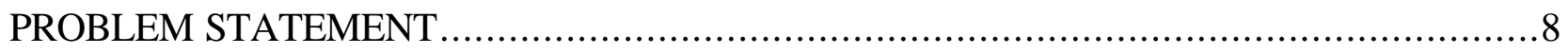

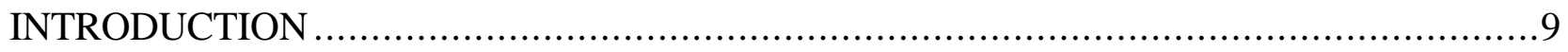

CHAPTER I - THE SEISMIC EXPERIMENTS ............................................... 11

I.1 THE SURFACE SEISMIC ................................................... 11

I.1.1 The principle of the seismic methods.........................................11

I.1.2 The $3 \mathrm{~d}$ seismic ............................................................... 16

I.1.3 The resolution .................................................................... 18

I.1.4 The seismic attributes ......................................................23

I.1.4.1 The history and importance of the seismic attributes ........................23

I.1.4.2 The acoustic impedance...................................................... 24

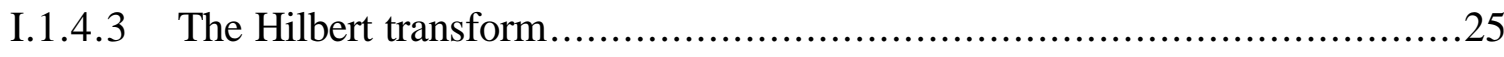

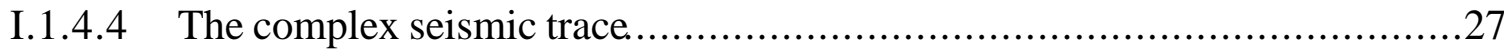

I.1.4.4.1 The basics of the instantaneous attributes ..............................29

I.1.4.4.2 The trace amplitude ............................................... 31

I.1.4.4.3 The instantaneous phase............................................ 31

I.1.4.4.4 The instantaneous frequency ......................................... 32

I.1.4.4.5 The trace envelope......................................................... 32

I.2 THE CROSSWELL SEISMIC ....................................................... 33

I.2.1 Description and comparison with other industrial applications.....................33

I.2.2 The traveltime inversion problem ................................................36

I.2.2.1 The theory of the traveltime inversion .................................... 36

I.2.2.2 The nonuniqueness problem, constraints................................. 39

I.2.3 The complexity of the raypaths in the crosswell seismic experiments ...............41 
CHAPTER II - ARTIFICIAL NEURAL NETWORKS 45

II.1 DESCRIPTION AND SIMILARITIES WITH THE HUMAN LOGIC ................45

II.2 NEURAL NETWORKS VERSUS CONVENTIONAL COMPUTERS................48

II.3 GENERAL REGRESSION NEURAL NETWORK....................................50

II.4 NEURAL NETWORKS APPLICATIONS IN THE PETROLEUM INDUSTRY .....54

CHAPTER III - DIFFERENT WAYS TO ANALYZE THE SEISMICEXPERIMENTS..........58

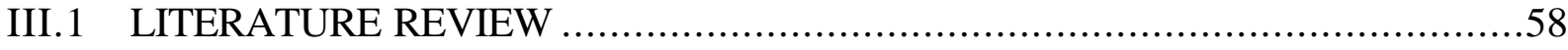

III.1.1 Different ways of analyzing the seismic experiments............................58

III.1.2 The nonuniqueness of the traveltime tomography ............................62

III.1.3 The prediction of reservoir properties in depth ................................69

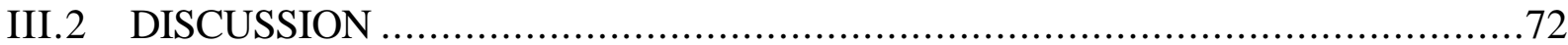

III.3 WHY A DIFFERENT METHODOLOGY FOR TRAVELTIME TOMOGRAPHY IS REQUIRED? ...................................................................... 73

CHAPTER IV - THE FIELD DATA ................................................................ 75

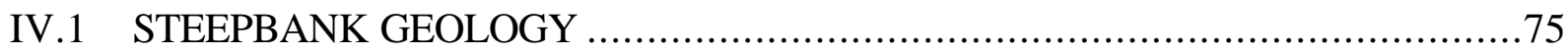

IV.2 HASDrive process............................................................... 77

IV.3 THE SURFACE SEISMIC EXPERIMENT.......................................... 78

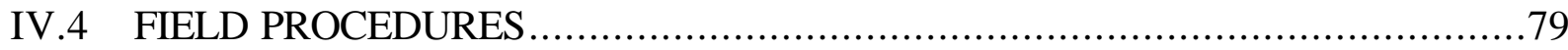

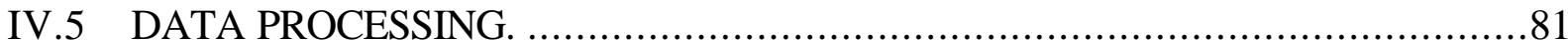

IV.6 SYNTHETIC SEISMIC TRACES .............................................. 82

IV.7 TRACE ATTRIBUTES ........................................................ 83

IV.7.1 Attributes computed........................................................ 83

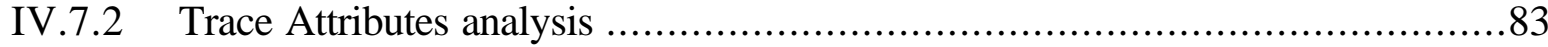

IV.7.2.1 Conventional Amplitudes................................................83

IV.7.2.2 Acoustic Impedance.................................................... 84

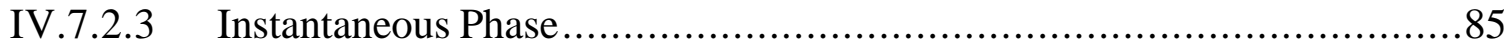

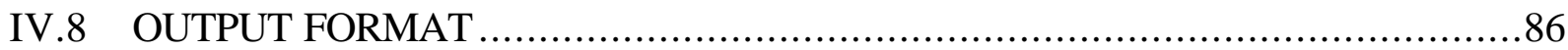

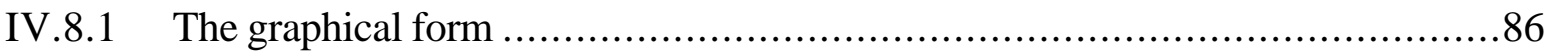

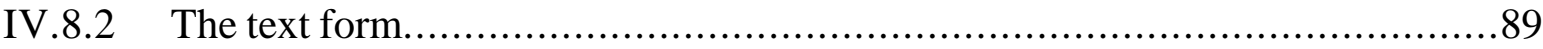

IV.9 LOGS AVAILABLE .................................................................91 
IV.10 THE RESOLUTION OF THE CROSSWELL SEISMIC AND THE SURFACE SEISMIC EXPERIMENTS ............................................................... 92

IV.11 CHOICE OF THE ZONE TO BE ANALYZED..................................93

CHAPTER V - RESULTS................................................................. 95

V.1 CHARACTERIZATION OF THE INTERWELL SONIC VELOCITIES USING

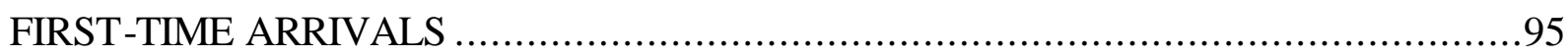

V.2 THE MAPPING CROSSWELL SEISMIC- SURFACE SEISMIC .....................98

V.2.1 The input values...............................................................98

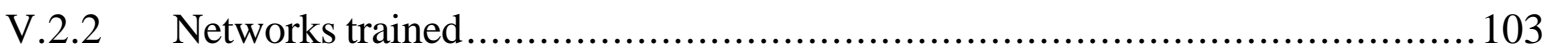

V.2.3 Networks results........................................................... 106

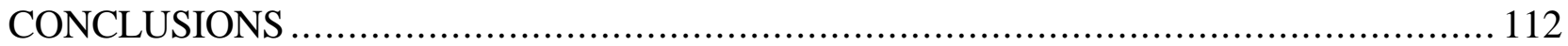

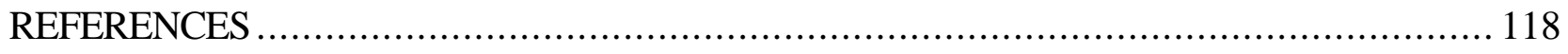

APPENDIX A- The trace attributes plot ..................................................... 125

APPENDIX B The data spectra for the surface seismic and for the crosswell seismic for CH1$\mathrm{CH} 3$. These spectra are also representative of the $\mathrm{CH} 2-\mathrm{CH} 4$ sections.

Appendix C - COMPARISON BETWEEN THE REAL TRACE SHAPE IN THE SURFACE SEISMIC AND THE CROSSWELL SEISMIC TRACE - trace 19, CH2- CH4 line Appendix D- Statistics on prediction goodness for training, calibration and verification datasets.

Appendix E The neural network prediction for the crosswell seismic traces - the verification set137 Appendix F: Crossplots of the seismic attributes: real values vs. predicted values (best network) 


\section{LIST OF FIGURES}

Figure 1 The map of the entire high- resolution characterization research........................ 2

Figure 2 The crosswell seismic gives information about a smaller rock volume when comparing to the surface seismic

Figure 3 The prediction of the envelope, as given by high-resolution crosswell seismic, using

low-resolution surface seismic

Figure 4 The experiment area....................................................................... 8

Figure 5 Schematic figures illustrating the principles of reflection seismology..................12

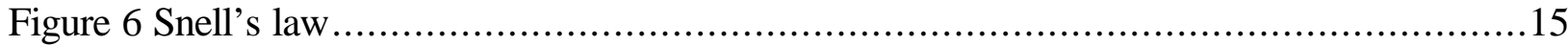

Figure 7 The minimum ray path of a ray ...................................................... 15

Figure 8 Average trace morphology for each of the 7 facies identified in .......................17

Figure 9 Average spectra for two pairs of wells (a) $25 \mathrm{~m}$ offset and (b) $75-\mathrm{m}$ offset.....................19

Figure 10 At a depth, the seismic attributes represent an average value for a volume that is smaller in the crosswell seismic than in surface seismic .20

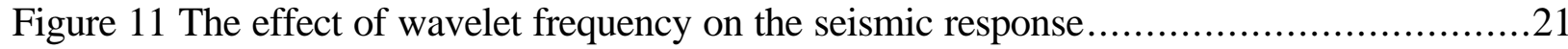

Figure 12 The acoustic impedance response of a low-impedance wedge in a high-impedance

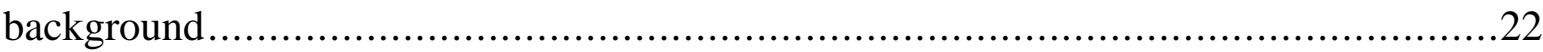

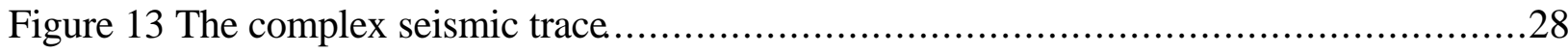

Figure 14 The vectors in the seismic attributes analysis .....................................29

Figure 15 The instantaneous -amplitude function calculated from a complex seismic trace.......32

Figure 16 The crosswell seismic data acquisition................................................. 34 


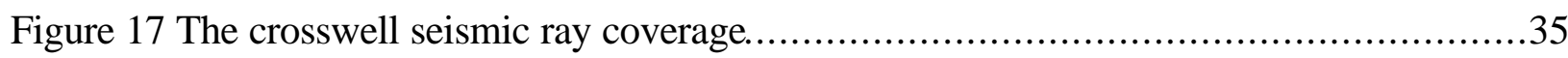

Figure 18 A simple single- interface model to illustrate some of the many seismic models.......41

Figure 19 A space-time record for a simple single-interface model shown in Figure 18 ..........42

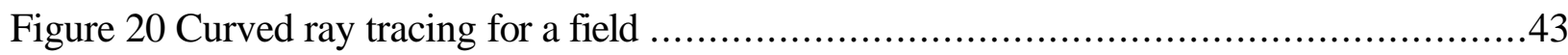

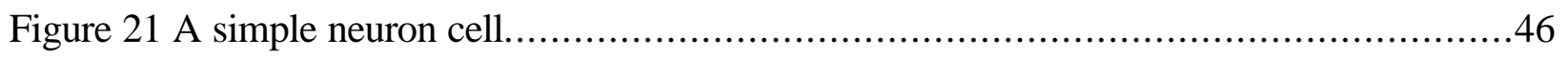

Figure 22 A schematic diagram of a neuron.............................................................

Figure 23 The estimated parent density function for a small value of the smoothing factor.......52

Figure 24 The estimated parent density function for an intermediate value of the smoothing

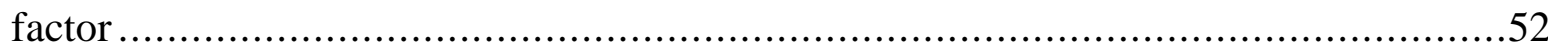

Figure 25 The estimated parent density function for a larger value of the smoothing factor ......53

Figure 26 Prediction of the core permeability using Neural Networks methods, in comparison to

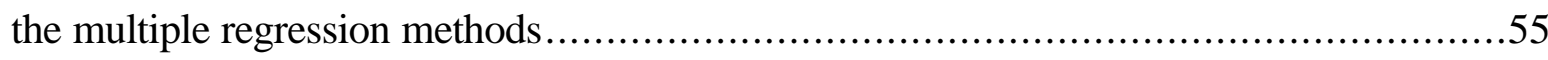

Figure 27 Seismic velocity as estimated by the neural network as a function of pressure and saturation .57

Figure 28 Plot of porosity versus slowness for 256 wells used in deriving velocity- porosity

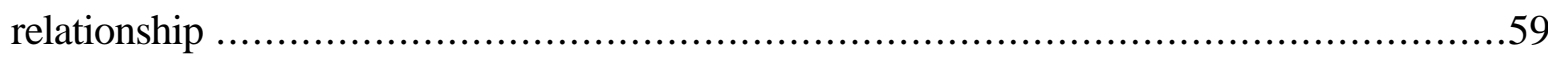

Figure 29 Comparison of MUN and Amoco tomograms.................................................64

Figure 30 Comparison of BOMTOM and U. Tulsa tomograms ......................................64

Figure 31 Comparison of two models regarding the line $\mathrm{CH} 2-\mathrm{CH} 4$ in the Steepbank crosswell

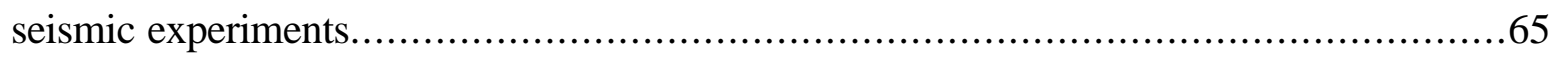

Figure 32 Instantaneous frequency attribute against the volume of clay log property .............67 Figure 33 The spatial distribution of the volume fraction of the clay, with and without the seismic .67 
Figure 34 A comparison of estimation errors at each of the six validation wells for effective porosity (left) and water saturation (right) with and without seismic guidance.................68

Figure 35 Core porosity and gamma ray correlation................................................ 70

Figure 36 Comparison of actual and neural network Gamma Ray log for two wells predicted in ..........70

Figure 37 The neural network approach to predict the lithology in ................................. 71

Figure 38 The main goal of the research in the petroleum area: use the crosswell seismic to predict the rock properties using its better resolution compared with surface seismic and the

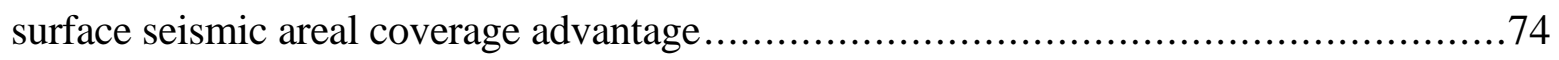

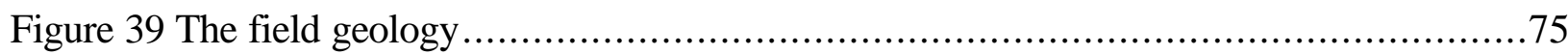

Figure 40 Sonic log velocities and typical wave path from one well to another .....................76

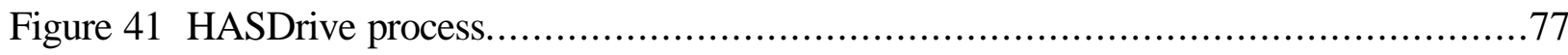

Figure 42 Map of 3-d surface survey and wells used in crosswell survey. Small numbers are receiver locations; medium size numbers are shot locations.....................................78

Figure 43 The synthetic traces for the $\mathrm{CH} 2-\mathrm{CH} 4$ line, repeated 10x...............................87

Figure 44 The format of the text files available for the project..........................................89

Figure 45 The area taken for the mapping surface seismic- crosswell seismic ......................94

Figure 46 The shape of the sonic log obtained from Geotom3D compared with the real values-

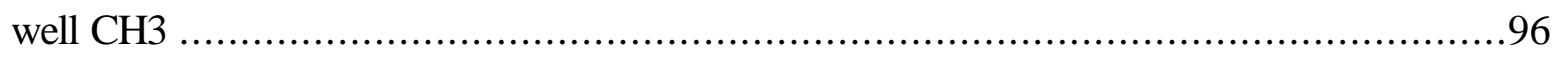

Figure 47 GEOTOM3D model for CH1-CH3 area.....................................................

Figure 48 The well position and the traces notation for the project...................................99

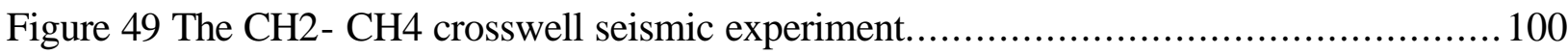

Figure 50 The $\mathrm{CH} 1$ - CH3 crosswell seismic experiment............................................... 101

Figure 51 Average R2 of the training sets for different networks................................. 107 
Figure 52 Average R2 of the calibration sets for different networks............................. 107

Figure 53 Average R2 of the verification sets for different networks........................... 107

Figure 54 The network predictions for the crosswell seismic traces - the best prediction (left) for the envelope and the worst prediction (right) for the instantaneous phase- trace $30, \mathrm{CH} 2$ -

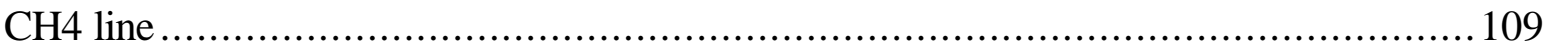

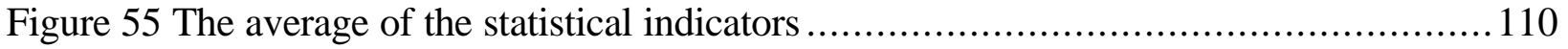




\section{LIST OF TABLES}

Table 1 Properties of tomography software used in the Glennpool field ............................63

Table 2 The Excel file used in the mapping surface- crosswell seismic process ....................90

Table 3 Logs and depth intervals available for the Steepbank field ...................................91

Table 4 The choice of traces and input/output in the ANN analyzed ................................ 105 


\section{EXECUTIVE SUMMARY}

The global objective is to develop a methodology for high resolution reservoir characterization. High resolution reservoir characterization is defined as a process that allows engineers and scientists to generate a volumetric picture of rock and fluids characteristics at any location in the reservoir in the scale of wireline logs.

The methodology provides a piece of a complex puzzle in the next generation of "intelligent" tools that can identify the nonlinear relationship between seismic data and reservoir characteristics as described through the magnetic resonance imaging process. The model would be used to construct a 3-D visualization of rock and fluid properties, and perhaps other relevant reservoir information away from the wellbore, knowing the 3-D seismic data.

The approach proposed to meet the project objectives included three separate but closely related steps as shown in below- Figure 1. The first step consists of building a solid and robust relationship between conventional well logs and reservoir characteristics such as effective porosity, permeability and fluid saturation as determined by MRI logs [39]. The second step involves the development of a correlation between high-resolution seismic (crosswell seismic) and conventional well $\log s$ such as gamma ray, density and resistivity logs. The third step involves developing an algorithm to deconvolve "low" resolution 3-D seismic data to a "high" resolution crosswell seismic. The end result would be a relationship that allows MRI-quality rock and fluid property information to be generated in a continuous vertical and horizontal manner along all available 3-D seismic lines.

This global objective is achieved in a three-step process. This study is concentrated on one of the three steps involved in this process. 


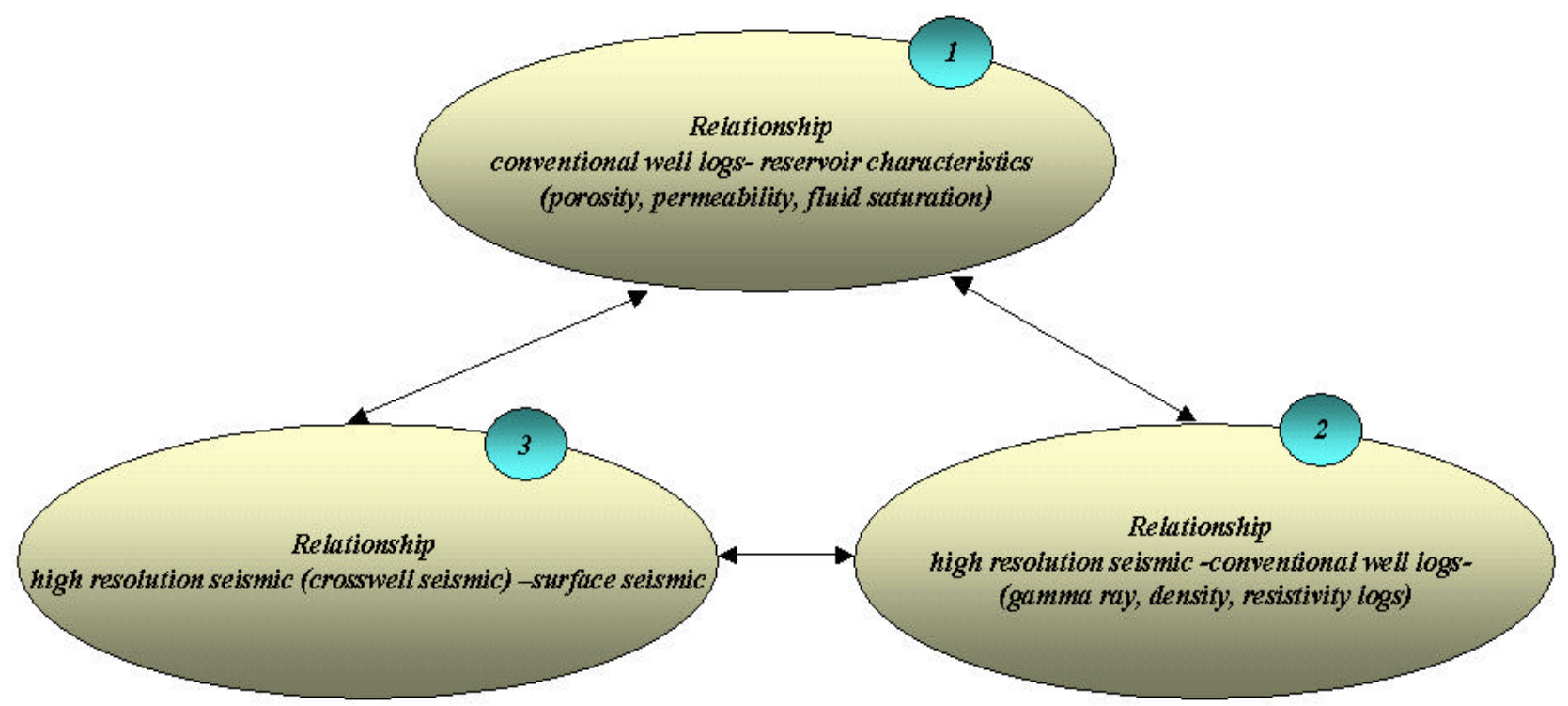

Figure 1 The map of the entire high- resolution characterization research

The objective of this project is to provide the means to fulfill step three of the aforementioned process by proving that crosswell seismic traces can be predicted from surface seismic experiments.

Crosswell seismic interpretation is a relatively new method that has the capability to increase the accuracy of the reservoir characterization. Unfortunately the method is expensive and the experiment must be conducted with care. Fortunately this situation was encountered at the Steepbank seismic surveys, conducted by Chevron Oil Company in the Alberta tar sands, Canada.

The methodology of the classical seismic methods (surface seismic) is well defined and, comparing with crosswell seismic has an enormous advantage: it covers a large portion of the field while the crosswell seismic covers only a plane from one well (source) to another (receiver). 
The disadvantages of the surface seismic is the fact that the resolution is much lower that the resolution of the crosswell seismic- Figure 2. In this relatively shallow field (300 meters) the resolution of the surface seismic is half the resolution of the crosswell seismic. In deeper fields the situation is worse- traveling downward, the signal lose more and more its high- frequencies.

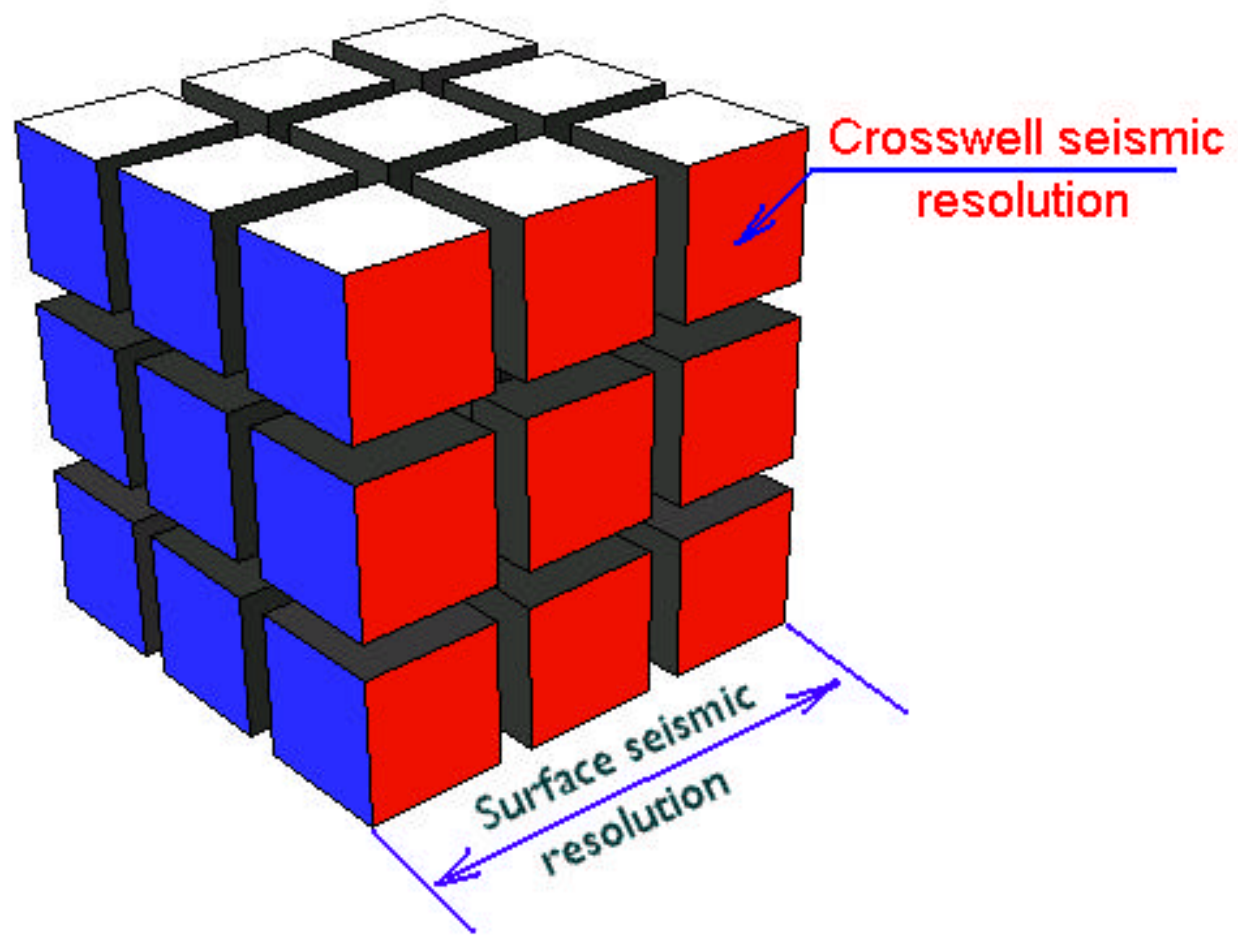

\section{Figure 2 The crosswell seismic gives information about a smaller rock volume when comparing to the surface seismic}

If a correlation could be created between surface seismic and crosswell seismic there would be a big advantage: everywhere in the area analyzed by surface seismic, the crosswell seismic can be predicted and it characterized by a better resolution. Also, the crosswell seismic traces can be useful as an input in a second neural network (input the surface seismic and the crosswell seismic and, as output, reservoir characteristics)- the subject is still under research.

This project shows that this mapping is possible. The results obtained here were very good. Having the surface seismic traces and the traces location we were able to predict with a very good accuracy the crosswell seismic traces determined for the various surface seismic attributes (Acoustic impedance, Conventional amplitudes, Instantaneous phase, Instantaneous frequency, Trace envelope). 
For example, for the best network, the predicted and actual envelope for trace 30, $\mathrm{CH} 2-\mathrm{CH} 4$ is shown in Figure 3. The figure shows one of the seismic traces from the verification set as well as the neural network prediction.

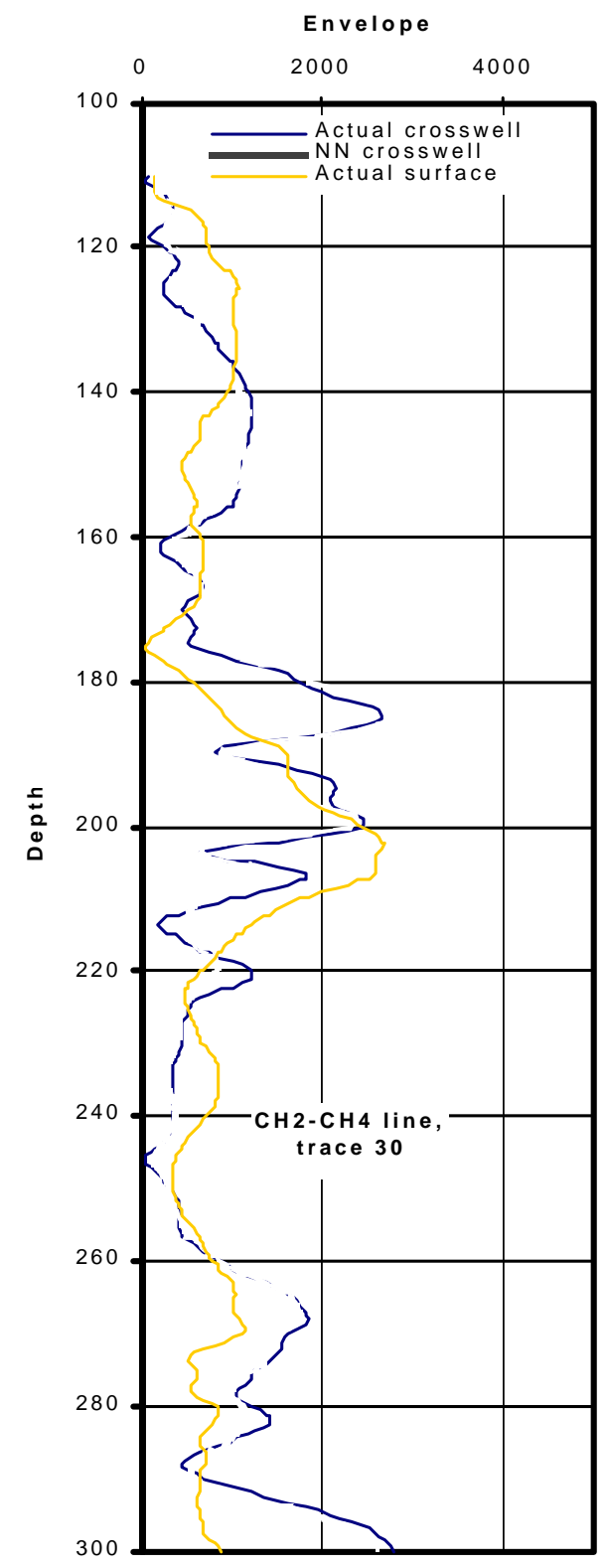

Figure 3 The prediction of the envelope, as given by high-resolution crosswell seismic, using low-resolution surface seismic 
This study shown that, irrespective of the traces chosen for training, calibration or verification, the performance of the networks is approximately the same and our methodology is very robust.

This project tried to predict the crosswell seismic traces using a neural network. In the training and testing sets the information used as input were five seismic attributes and their spatial location (x, $\mathrm{y}$ and $\mathrm{z}$ ). The output was represented by 5 crosswell seismic attributes computed from reflections. For the best network, the statistical comparison between the real and the predicted values shown also that the mapping is possible:

\begin{tabular}{|l|c|c|}
\hline \multicolumn{1}{|c|}{ Seismic attribute } & R squared & Correlation coefficient $^{*}$ \\
\hline \hline The acoustic impedance & 0.8113 & 0.9025 \\
\hline The trace amplitude & 0.7509 & 0.8683 \\
\hline The instantaneous phase & 0.5028 & 0.7114 \\
\hline The instantaneous frequency & 0.7424 & 0.8622 \\
\hline The trace envelope & 0.8631 & 0.9291 \\
\hline
\end{tabular}

* the verification set 


\section{OBJECTIVE}

The objective of this project is to correlate the surface seismic traces to the crosswell seismic traces. Since this process involves mapping of a low resolution image into a higher resolution image, it inherently is a problem with non-unique solutions. In such cases development of analytical solutions are quite difficult. Therefore a virtual intelligence approach has been chosen to address this deconvolution problem.

In seismic interpretation, it is well known that the resolution of the interpretation is poor. It means that beyond a lower limit of the dimension of an object located below the soil, its existence and/or shape cannot be well defined.

A new technology is now available: crosswell seismic. We will not cover here VSP and single well seismic. The acoustic rays are emitted and received into the wells. The experiments are expensive and require extensive care in design and in data acquisition but the results obtained by now show that this method can improve the reservoir definition.

The literature review covered for this project has not shown cases where such crosswell seismic experiments were performed in the entire field. Where applied, the crosswell seismic covered only a small portion of the field- only few wells. From one experiment the interpreter has information about a zone in the field that is much smaller that the entire field. Thus, even if the interpretation would be unique, the lack of experiments over the entire field make it improbable to find an accurate characterization of the reservoir based on the crosswell seismic experiments.

Surface seismic is easier to perform than the crosswell seismic - it is a common job. Usually the method is cost effective to perform it at the field scale but, unfortunately, its resolution is much lower compared to the crosswell seismic resolution, especially in deep fields. 
Even if we don't consider difference in the resolution between these two seismic methods, toward a proper reservoir definition and reservoir management, all the information regarding the field should be taken into consideration. Neglecting the crosswell seismic results (if they are available in the field) can lead to an unreal/coarse reservoir model, even if the necessary information to improve it is at hand.

It is still under research if the method covered here give accurate results at the field scale. If the reservoir characteristics will be used as inputs in a reservoir simulator and the history matching process is shorter than usual, then the methodology presented here will be validated.

If a mapping of surface seismic-crosswell seismic could be created then, having the surface seismic, the crosswell seismic would be created without the need to perform this expensive analysis through the field. In other words, this process can be named "generation of synthetic crosswell seismic traces from surface seismic traces". 


\section{PROBLEM STATEMENT}

The data for this project was obtained from an experiment performed in Canada by Chevron. The field is located about $60 \mathrm{~km}$ northwest of Fort McMurray, Alberta.

For the exploitation of the tar sands, a HASDrive evaluation was performed. The acronym stands for $\underline{\text { Heated }} \underline{\text { Annulus }} \underline{\text { Steam }} \underline{\text { Drive. }}$. The process circulates steam through the annulus of a horizontal well in order to heat a zone of the reservoir surrounding the well.

The pilot site consists of a five-spot pattern, shown in Figure 4. One of the wells served for the steam injection in this pattern- IN1.

In the crosswell seismic experiment the sources was mounted in the wells $\mathrm{CH} 1$ and $\mathrm{CH} 4$ and the receivers was cemented in the wells $\mathrm{CH} 2$ and $\mathrm{CH} 3$.

The crosswell seismic experiment gave a total of 4 crosswell sections: $\mathrm{CH} 1-\mathrm{CH} 2, \mathrm{CH} 1-\mathrm{CH} 3$, $\mathrm{CH} 4-\mathrm{CH} 2$, and $\mathrm{CH} 4-\mathrm{CH} 3$. Only the two diagonal crosswell sections $\mathrm{CH} 1-\mathrm{CH} 3$ and $\mathrm{CH} 4-\mathrm{CH} 2$ are analyzed here.

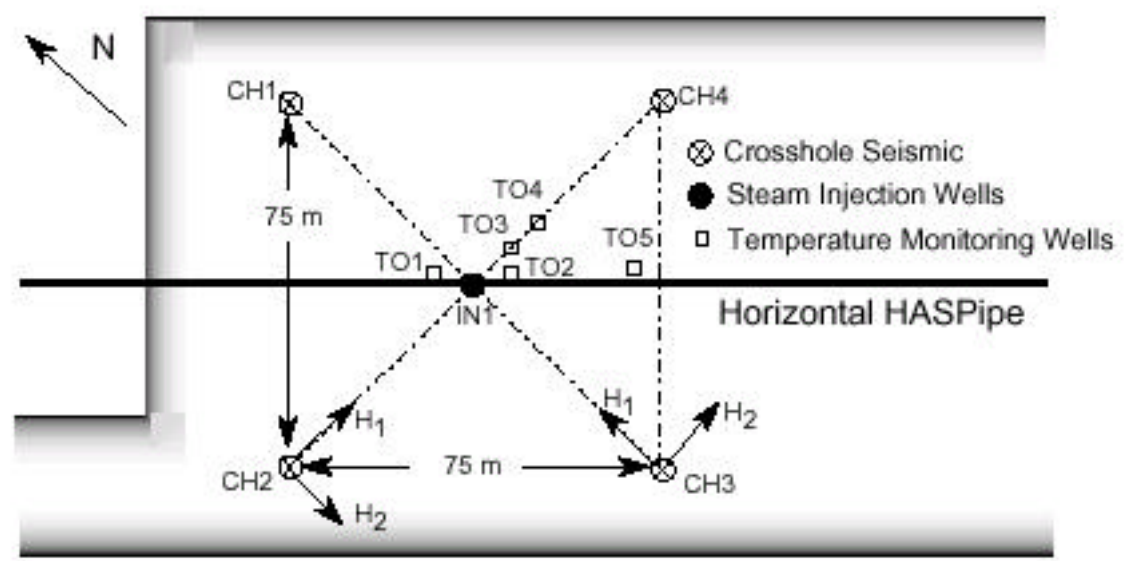

Figure 4 The experiment area

The surface seismic was interpreted to give data across the same directions: $\mathrm{CH} 1-\mathrm{CH} 3$ and $\mathrm{CH} 4-$ $\mathrm{CH} 2$ in order to make the deconvolution possible. 


\section{INTRODUCTION}

Reservoir engineers struggle daily to estimate reservoir properties on a spatial scale from wellbore data. This is true even though geostatistical methods have helped reduce the uncertainty associated with spatial predictions from pseudo-points support (wells). The current motivation for using seismic data, 3D or otherwise, to predict reservoir properties is due to the inherent large areal coverage of the seismic measurement.

The trade-off between the seismic survey, which allow excellent areal coverage, and the wellbore measurements is the vertical resolution. Wellbore measurements, such as logs, tend to have higher vertical resolution, to the order of few inches. Surface seismic surveys, which require acoustic energy to be transmitted over large depths, are plagued with poor vertical resolution. Between them a new method can be taken into consideration- the crosswell seismic. Due to its resolution (better that the surface seismic but worse than the logs resolution) the pertinent question is: Can we exploit and link the obvious advantages- vertic al resolution and areal coverage of the logs and surface seismic to our benefit using the crosswell seismic experiments? The current project shown that the trial worth the effort.

Acknowledging that researchers are far from explicitly correlating surface and crosswell seismic attributes, we relied on the capability of the Artificial Neural Networks to extract the non- linear relationship that correlates surface seismic properties with the crosswell seismic properties.

The seismic methods have made great inroads into the field of exploration over the last 35 years. It is now an indispensable exploration technique. Unfortunately the seismic methods suffer from two factors- the resolution and the accuracy of the interpretation.

Because the distance between wells is usually smaller than the reservoir depth and the seismic rays don't travel in the surface rocks or long distance the image of the analyzed area can be closer to the reality. Unfortunately the interpreting methods were not developed as the technology did. These methods are, by now, less reliable that surface seismic methods. 
The potential advantage of using the crosswell seismic is huge and the research tries to find the most suitable interpretation method.

The Athabasca Tar Sands in northern Alberta (the Steepbank seismic surveys) is a research field where both the crosswell seismic and surface seismic was performed. The quality of data acquisition creates conditions for a good interpretation technique.

The Steepbank seismic surveys were part of an experimental enhanced oil recovery project conducted by Chevron Oil Company in the Alberta tar sands. The site location is about $60 \mathrm{~km}$. northeast of Fort McMurray, Alberta. The project circulated steam through a closed horizontal pipe. The seismic surveys were conducted to monitor the changes in the field temperatures.

It was proven that the seismic velocities decrease with increased temperature in heavy oil, unconsolidated sand, or sandstone reservoirs. Thus any velocity reduction between the surveys would indicate the distribution of increased temperature and, by interference, the flow path the steam has taken.

Two crosswell and 3-D surface seismic surveys were recorded: the first in September 1991 prior to experiment, the second was acquired 7-14 January 1992 after 72 days of continuous steam injection.

Data analyzed in this project was extracted from just the first survey since the reservoir was unaltered by steam injection and in the same state as that which existed during well logging. 


\section{CHAPTER I - THE SEISMIC EXPERIMENTS}

\section{I .1 THE SURFACE SEI SMI C}

\section{I.1.1 The principle of the seismic methods}

The goal of exploration seismology is to find oil and gas reservoirs by seismically imaging the earth's reflectivity distribution.

Reflection seismology is the most powerful geophysical method to imagine the underground and is based on the artificial wave sources.

The seismic reflection is based upon the principle that if an impact is introduced at the surface, using a source, acoustic waves will spread throughout the subsurface until they are reflected, or bounced back, to the surface when material with a different acoustic impedance is encountered. The technique is based on determinations of the time interval that elapses between the initiation of a sound wave and the arrival of reflected or refracted impulses at one or more seismic detectors.

The results of a seismic survey may be presented in the form of a cross-sectional drawing of the subsurface structures as if cut by a plane through the shot point, the detector, and the center of the Earth. Such drawings are called seismic profiles.

Sensors, called geophones onshore (hydrophones offshore), located at the surface receive the impulses. A seismograph is used to record the data. On land the geophones respond to particle velocity or acceleration. Offshore, the hydrophones measure pressure.

The geophones are high fidelity particle velocity sensors that are capable of recording ground motions with a flat frequency response of 30 to $600 \mathrm{~Hz}$ [41]. The devices are coupled to the 
ground via spikes or bonded to pavement. They produce voltages proportional to the particle velocity by virtue of a moving coil suspended about a fixed magnet.

The basic principles of the reflection seismology are shown in the Figure 5. The collection of raypaths shown here are those associated with CDP gathers.
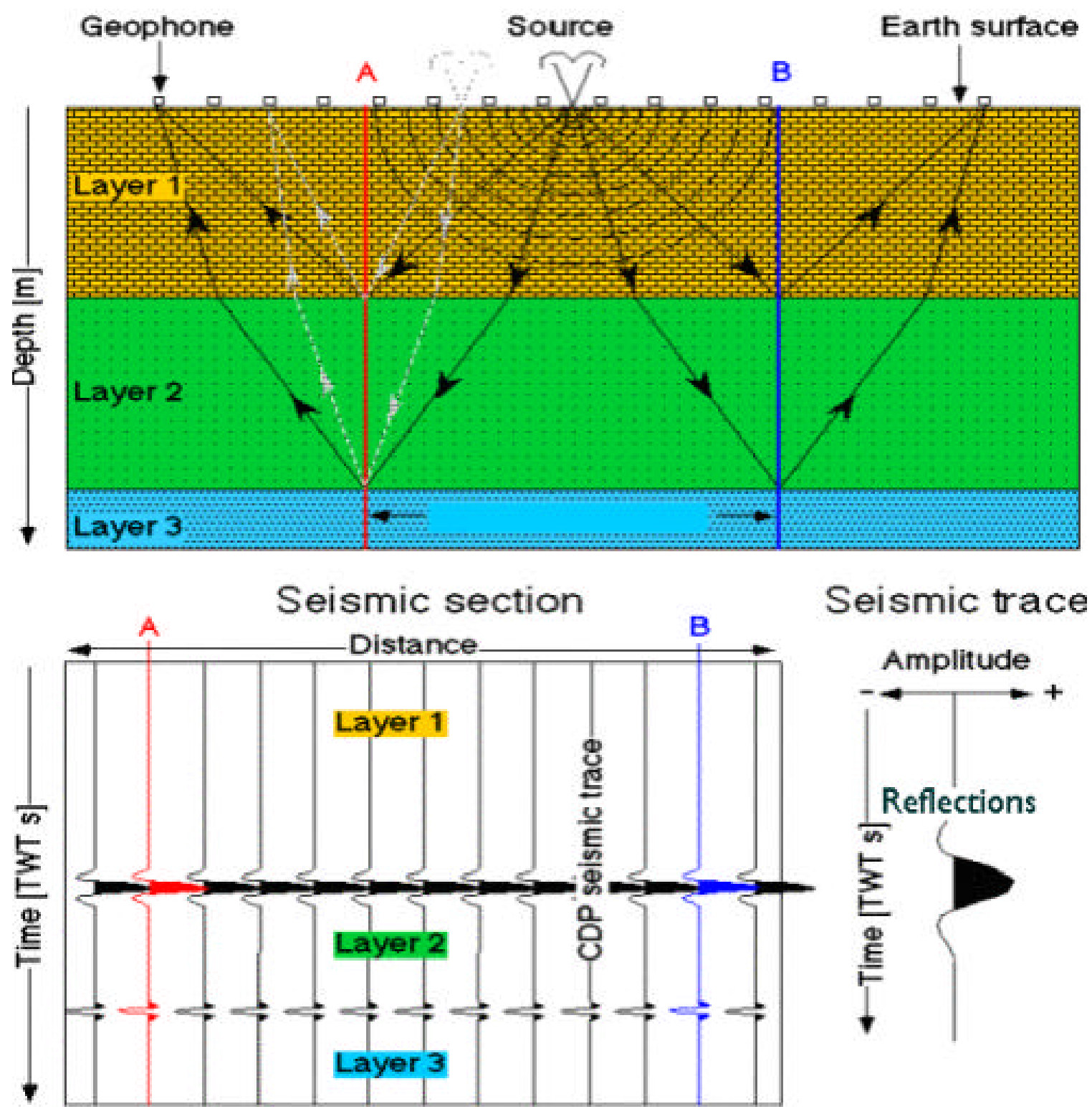

Figure 5 Schematic figures illustrating the principles of reflection seismology 
Usually, it all begins with a bang generated, for example, by a dynamite explosion on land or an air gun offshore, which sends a short, sharp pulse of sound into the ground.

The sound wave rushes down and down until it meets a new rock layer of hardness (hardness in the sense of the rock's resistance to being squeezed) different from the hardness of the rocks in which it is traveling (a different Poisson ratio). A replica of the downward-traveling sound wave echoes back toward the surface from the boundary between the two rock layers.

The original pulse continues its downward journey, gradually becoming weaker, sending echoes back to the surface every time it encounters a change in rock hardness. The greater the hardness change, the stronger is the echo. Listening devices (geophones on land and hydrophones offshore) hear the echoes as they return to the surface.

The seismic pulse will continue its downward journey into the Earth with constant velocity so long as the acoustic impedance of the rocks does not change.

Typically, however, the sedimentary sequence consists of successive layers of differing lithologies which also, as a rule, have differing acoustic impedance. This need not always be the case, as acoustic impedance is the product of two variables, velocity and density. It is quite common, for example, that a claystone and a relatively porous sandstone, although having quite different lithologies, have identical values for acoustic impedance.

When, however, the seismic wave encounters a rock layer with different acoustic impedance from the rock in which it is traveling, the wavefront splits. Part is reflected back toward the surface and part is transmitted and refracted to continue the downward journey. The wavefront split occurs exactly at the boundary between the different rocks and is caused by the abrupt change in acoustic impedance. The seismic-reflection method is based on the recording and measurement of reflections from such boundaries. 
There are usually so many echoes that, once they start arriving, they often overlap to form a continuous stream of sound. On a typical commercial seismic survey, the geophones listen for echoes for six seconds after the initial bang.

The last echoes to arrive are normally very weak, often one hundred thousandth of the strength of the early echoes; and so the geophones that detect them must be very sensitive.

Sound waves travel down to a reflector and back again; therefore, the time taken from the initial bang to the recording of a reflection is called two-way time.

If the overlying layer is softer than the underlying layer (i.e., has lower acoustic impedance), the reflection is positive; if the upper layer is harder, the reflection is negative.

In the simplest seismic system, consisting of a source and one geophone, reflections are assumed to originate from subsurface points midway between the two. However, such a system is very susceptible to noise (i.e., all forms of unwanted sound, such as multiples, wind noise, etc.); and it was soon found that recording reflections from the same subsurface point for different source- togeophone spacings (offset) not only improved the strength of primary reflections but also resulted in a significant decrease in noise. This was termed as an improvement in the signal-to-noise (SIN) ratio.

A setup by which reflections are recorded from the same subsurface point with different source-togeophone offset is known as common-depth-point (CDP) or common-midpoint (CMP) shooting. Each common midpoint consists of two or more traces, the number of which determines the coverage or fold of the seismic record. For example, two traces for a common depth point produce 2 - fold or $200 \%$ coverage; 96 traces, 96 -fold coverage.

Whether on land or offshore, it would be cumbersome to record all of the data for each common midpoint before proceeding to the next, and so data are acquired in the most time-efficient manner. This results in a jumble of data which has to be reordered during processing. 
The CDP technique in a reflection method is that, when a seismic ray crosses a boundary between two formations of different velocities, then the ray is bent according to Snell's law - Figure 6 [31]

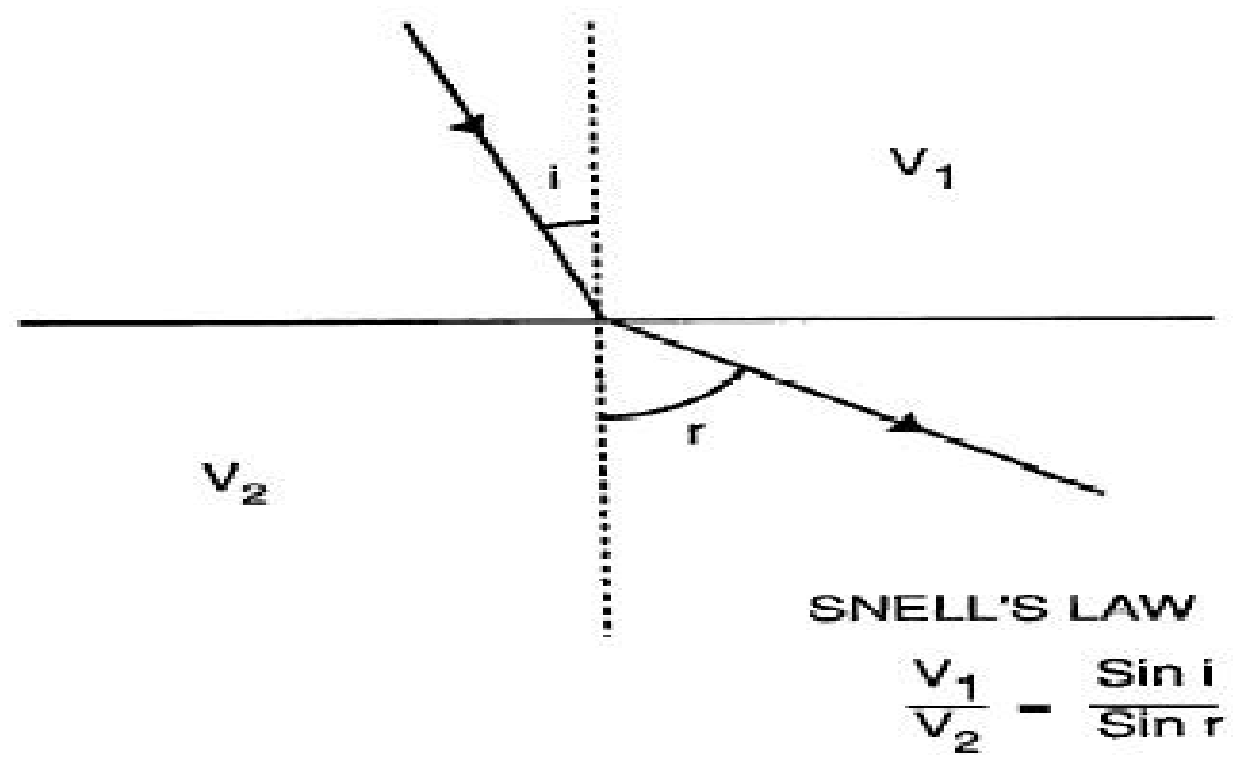

Figure 6 Snell's law

For a multilayer model raypaths are not straight rays but follow a minimum time path (Fermat's principle). For example in the Figure 7, a true raypath will follow a minimum time path ABCDE- it will travel less distance in the low-velocity layer and more distance in the higher velocity layer.

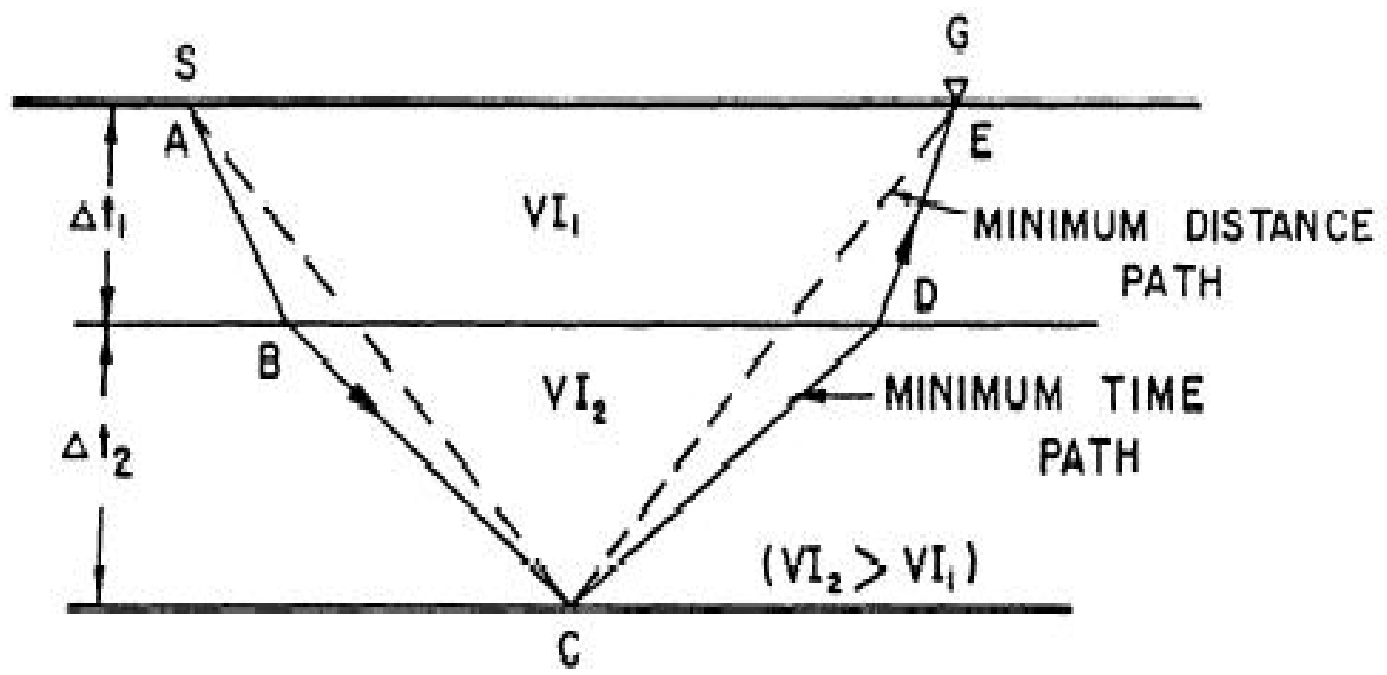

Figure 7 The minimum ray path of a ray 


\section{I.1.2 The 3d seismic}

In areas where structural traps are being explored, the subsurface beds generally dip in many directions. A 3-D survey can lead to a high degree of reliability in the interpretation because the volume of data provides more accurate and detailed sections [16].

Exploration for oil and gas involves the evaluation of a variety of information. Well logs provide detailed information at specific locations, usually an area less than one meter surrounding the well bore. We use our knowledge of formation signatures to recognize depositional environments and try to project this information between wells.

Reflection seismic methods can be used to create images of the geologic changes between wells. These images can help complete a picture of the subsurface that should enhance the ability of the explorationist to successfully select future well locations.

Seismic is an effective tool as long as it provides meaningful and helpful images for the desired objectives. The seismic tool must provide a cost efficient alternative to additional drilling.

2D seismic is recorded using straight lines of receivers crossing the surface of the earth. Acoustic energy is usually provided by the detonation of explosive charges or by large vibroseis trucks. The sound spreads out through the subsurface as a spherical wave front. Interfaces between different types of rocks will both reflect and transmit this wave front.

The reflected signals return to the surface where they are observed by sensitive microphones known as geophones. The signals detected by these devices are recorded on magnetic tape and sent to data processors where they are adjusted and corrected for known distortions. The final processed data is displayed in a form known as "stacked" data. 
In order to record data with sufficient density over large areas, we require a large number of recording channels. The operations of $3 \mathrm{D}$ are considerably more elaborate than $2 \mathrm{D}$ and the daily cost of crew is substantially increased. However, the rewards include fewer dry holes, more optimized well locations, guidance for horizontal drilling projects, more complete evaluation of mineral rights and better understanding of the nature of prospects.

3D reflection seismology differs from $2 \mathrm{D}$ profiling by the fact that data is gathered over a surface and not along a line. The data is processed into a cube, subdivided into bins formed by inlines and crosslines.

A better resolution can improve the field characterization. Journel and Derain [15] and Macrides, Journel and Dequirez [30] shown that the shape of the seismic trace can give information regarding the facies. In [30] they identified 7 facies, each of them having its own standard seismic trace- Figure 8. It is obvious that a better surface seismic resolution can lead to a better reservoir characterization and the use of the crosswell seismic can be the tool.

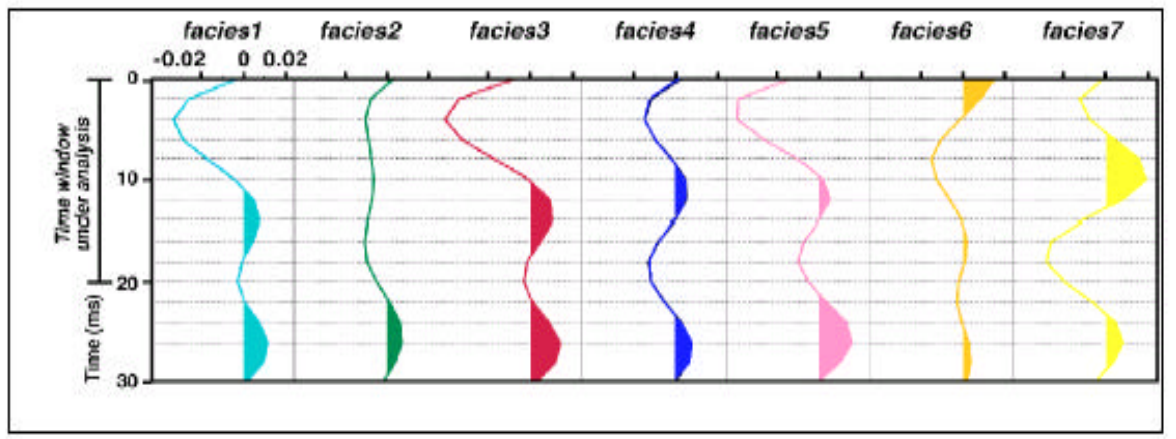

Figure 8 Average trace morphology for each of the 7 facies identified in [30] 


\section{I.1.3 The resolution}

The resolution involves the ability to distinguish that more than one interface is involved in a reflection. The resolvable limit is the minimum separation that two interfaces can have and still be recognized. It is not dependent on the background noise and the interpreters' ability to see minor changes in wave shape.

The generally accepted limit on vertical resolution is the Rayleigh resolution limit (1/4 of the dominant wavelength). The relation between frequency, velocity and wavelength is:

$$
\text { wavelength }=\frac{\text { velocity }}{\text { frequency }}
$$

A decrease in wavelength or the related increase in frequency will increase the spatial resolution of the data. Once the raypaths travel through the earth, the high frequencies are more attenuated that the low frequencies. It means that the resolution is decreasing with increasing depth. Thus, from a surface seismic experiment the near-surface layers are described with a more accuracy compared to the deeper layers located at a greater depth. The situation is similar for the crosswell seismic - the accuracy of interpreting is reduced when the wells are too far.

The concept of decreasing the dominant wavelength with increasing the travel distance is presented in Figure 9 for a crosswell seismic experiment where the where the frequency content is measured in two wells located at different distances from the source [44], at 25 and 75 meters. 
(a)

(b)
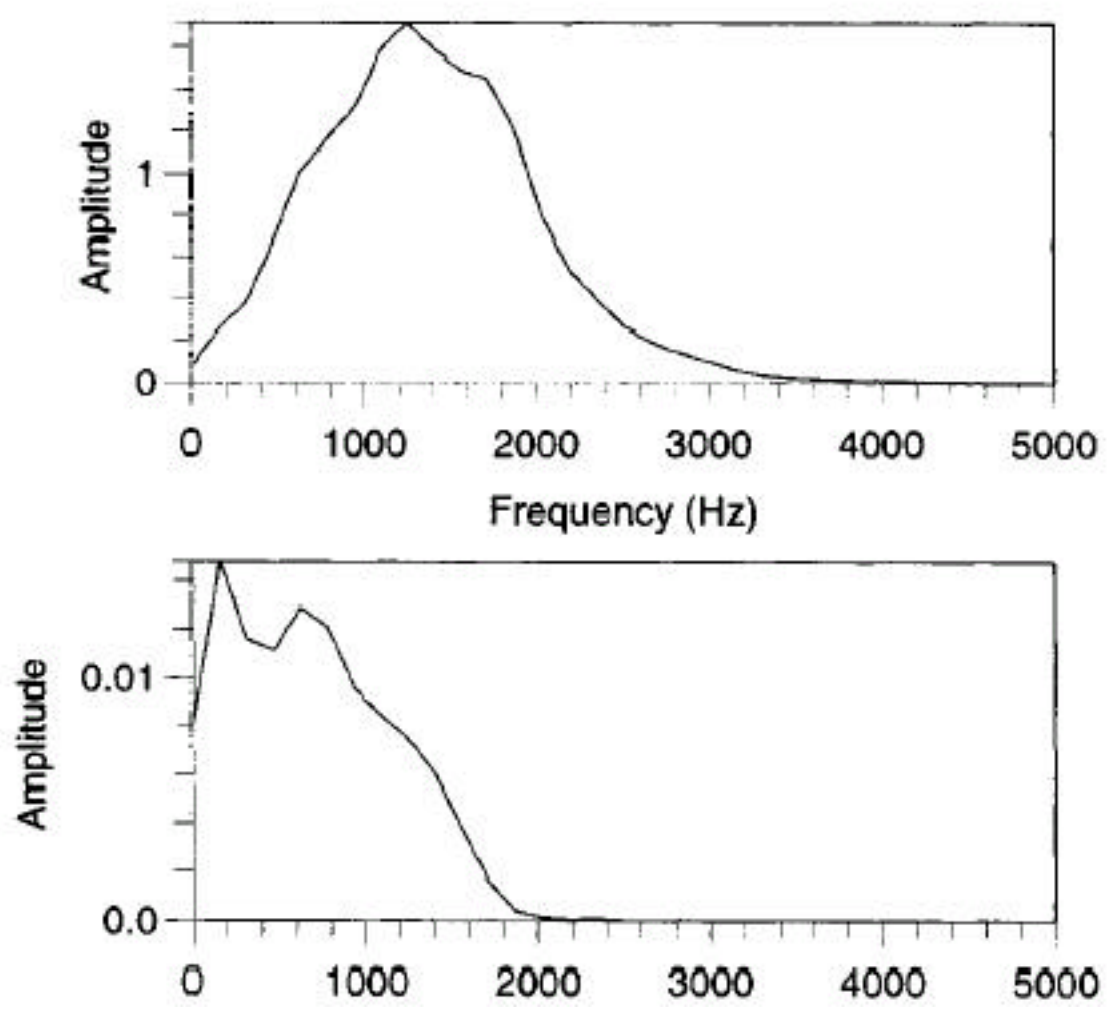

Figure 9 Average spectra for two pairs of wells [44] (a) $25 \mathrm{~m}$ offset and (b) 75-m offset

Figure 11 shows the effect of different wavelet length (frequency) on the seismic response [2] for a given stratigraphical column. The frequency content increases from Figure 11, wavelet "c", to Figure 11, wavelet "f". The wavelet that has a higher frequency content and a lower dominant wavelength- Figure $11 \mathrm{f}$ predict with a better accuracy the layers than the wave with a lower frequency content- Figure $11 \mathrm{c}$.

The resolution is directly influenced by the wavelength. The wavelength can be seen as influencing the size of a moving window. At each depth, the value predicted is actually an average value of all seismic attributes values that exists in the window- Figure 10. 
THE RESOLUTION OF THE CROSSWELL SEISMIC AND SURFACE SEISMIC

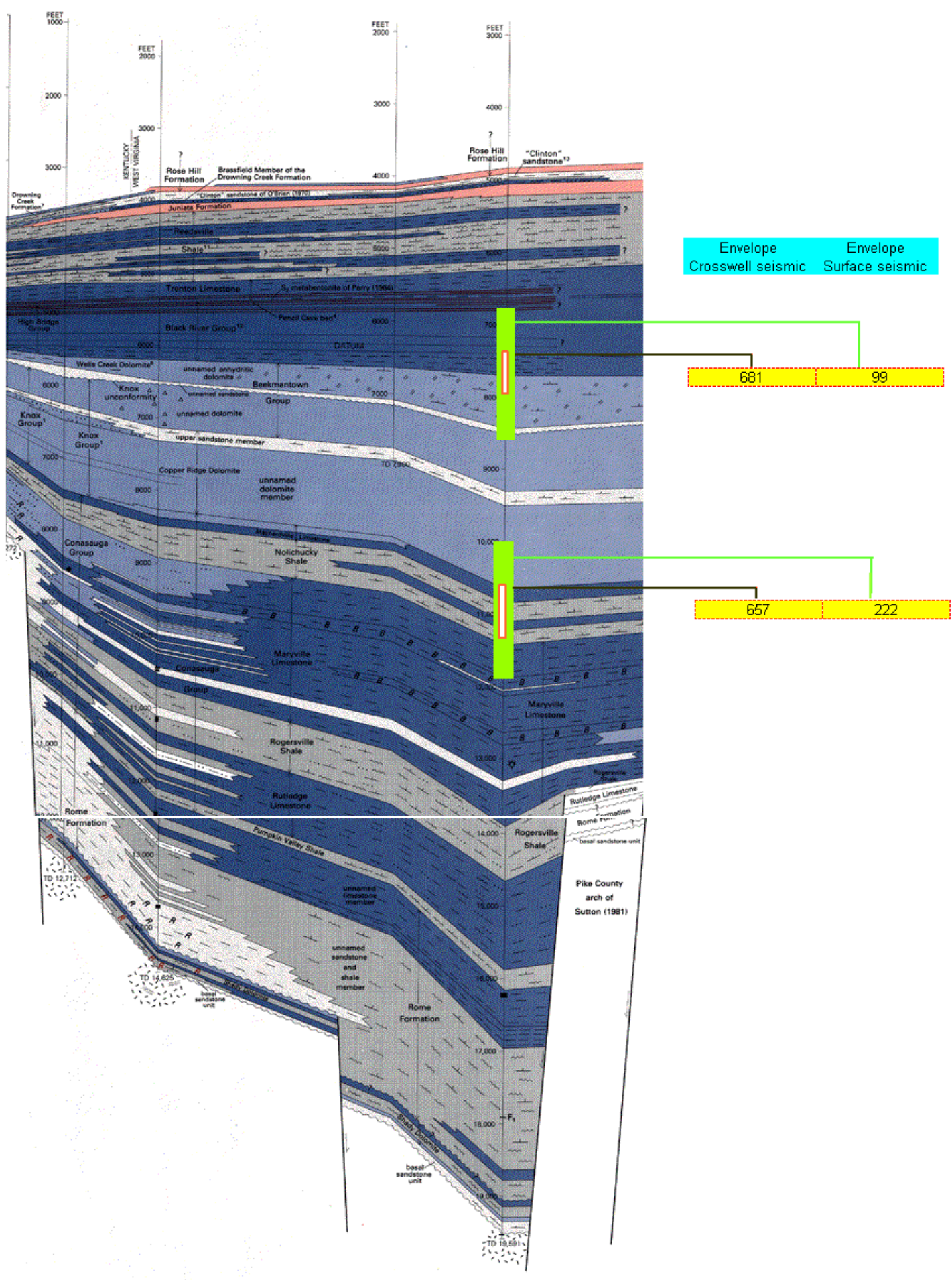

Figure 10 At a depth, the seismic attributes represent an average value for a volume that is smaller in the crosswell seismic than in surface seismic 


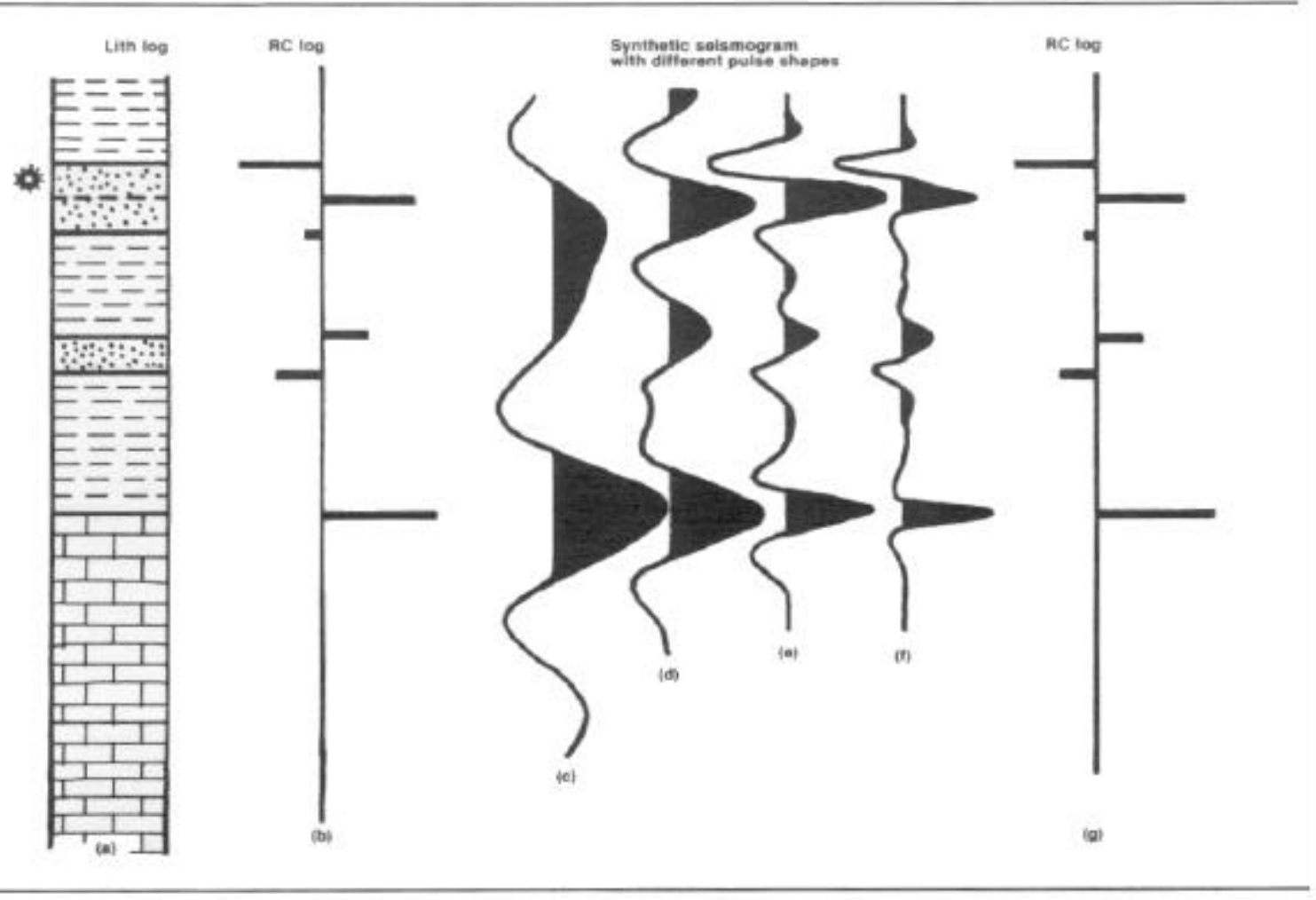

Figure 11 The effect of wavelet frequency on the seismic response [2]

The acoustic impedance response for a simple model of a low-impedance wedge in a highimpedance background [23] and [2] is shown in the Figure 12. Suppose that a limestone unit (high velocity) is encased in claystones (low velocity). A wavelet is reflected from the top of the limestone. The polarity of the incident wavelet is preserved on the reflected wavelet.

The reflection from the base limestone has its polarity reversed, as we would expect from a change from higher to lower acoustic impedance [2]. The two reflected wavelets of opposite polarity will be separated in time so long as the time thickness of the limestone is equal to, or greater than, half the wavelength of the seismic wavelet. Potentially we can resolve top and base limestone, so long as the limestone thickness is greater than half the wavelength. Otherwise the two opposite polarity reflections begin to overlap and interfere. 
When the two-way transit time of the limestone reaches half the wavelet width (i.e. the limestone thickness equals one-quarter of the wavelength) the two wavelets constructively interfere to form a single wavelet of anomalously high amplitude. This thickness is known as the tuning thickness.
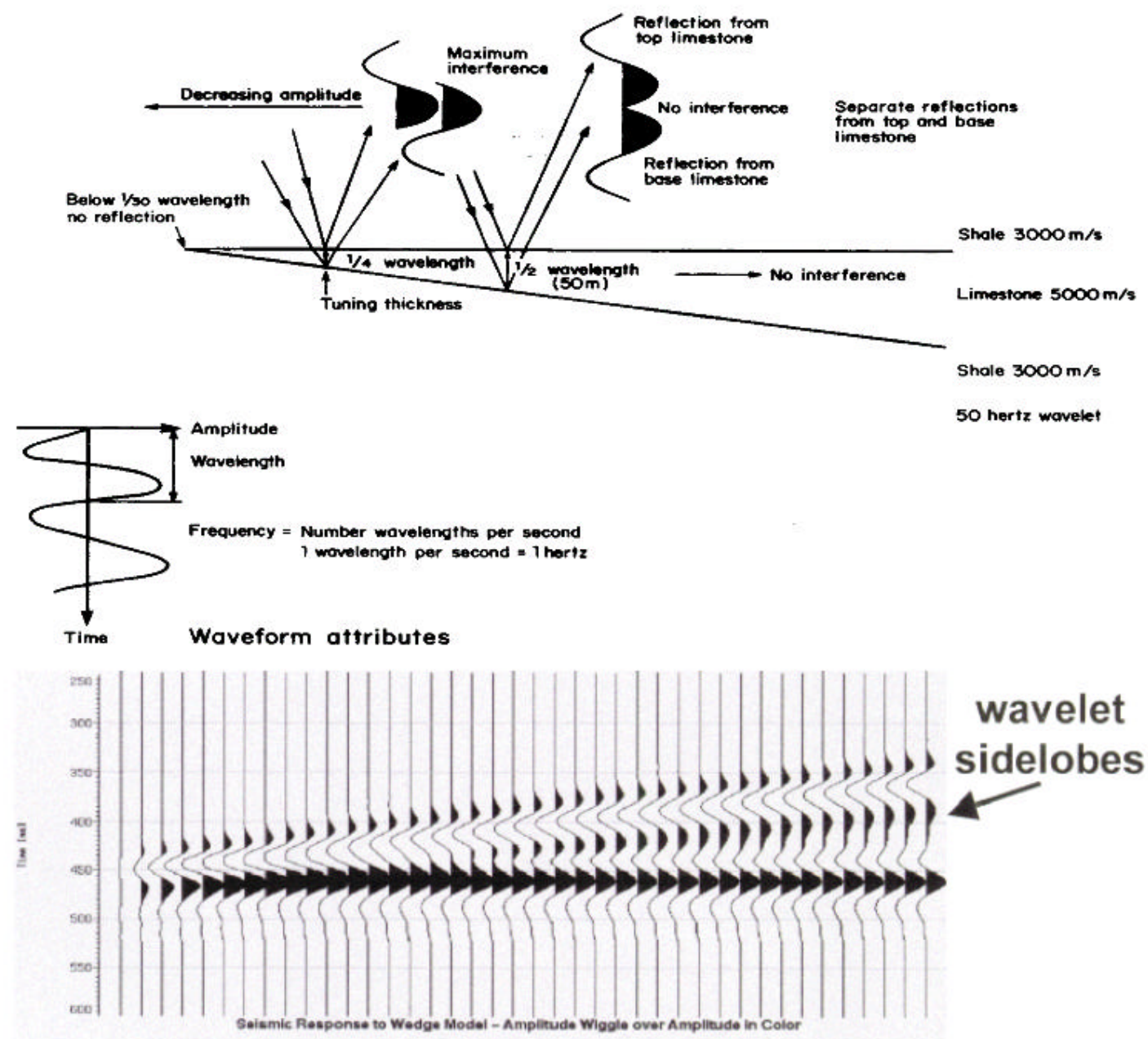

Figure 12 The acoustic impedance response of a low-impedance wedge in a high-impedance background [2] and [23] 


\section{I.1.4 The seismic attributes}

\section{I .1.4.1 The history and importance of the seismic attributes}

Seismic attributes are all the information obtained from seismic data either by direct measurements or by logical or experience based reasoning.

Complex seismic trace attributes were introduced around 1970 as useful dis plays to help interpret the seismic data in a qualitative way. Walsh published the first article in the 1971 issue of Geophysics under the title of "Color Sonograms". At the same time Nigel Anstey of SeiscomDelta had published "Seiscom 1971" and introduced reflection strength and mean frequency. Realizing the potential for extracting useful instantaneous information, Taner, Koehler and Anstey turned their attention to wave propagation and simple harmonic motion. This led to a landmark paper in the June 1979 issue of Geophysics by Taner, Koehler and Sheriff. At that time, amplitude, phase and frequency plus combinations of amplitude and phase, namely weighted average frequency and apparent polarity were first introduced.

Today, however, the explorationist is faced with a bewildering choice of hundreds of attributes and the almost insurmountable task of determining which of these attributes may offer greater insights into the petrophysical composition and geometry of the reservoir. 


\section{I.1.4.2 The acoustic impedance}

The predictable and characteristic acoustic properties of a rock are defined as its acoustic impedance $(\mathrm{Z})$, the product of density (p) and velocity (V):

$Z=\rho V$

The strength of a reflection generated at a boundary can be quantified in terms of the boundary's reflection coefficient (RC); at normal incidence this is:

$$
R C=\frac{Z_{2}-Z_{1}}{Z_{2}+Z_{1}}
$$

where:

$\mathrm{Z}_{1}=$ acoustic impedance in the upper layer

$\mathrm{Z}_{2}=$ acoustic impedance in the lower layer

The reflection coefficient can be positive or negative depending whether "softer" rocks overlie "harder" rocks or vice versa.

Velocity is usually more important than density in controlling acoustic impedance. For example, porosity variation or the content of the pore fluids (e.g., gas in a sand- stone) has a much more significant effect on velocity than on the density of the rock.

To convey a more tangible meaning to the concept of acoustic impedance, Anstey (1977) likened it to acoustic hardness. "Hard" rocks, for example, limestone, granite, etc., have high acoustic impedance, whereas "soft" rocks, for example, clays, are relatively squeezable and have low acoustic impedance. 
We do not actually measure directly the contrast in acoustic impedance across a boundary but deduce it from the amplitude of the recorded reflection. The greater the amplitude, the stronger the reflection and, by inference, the greater the acoustic-impedance contrast. In terms of amplitude the reflection coefficient is the ratio of amplitude of the reflected wave to that of the incident wave. For example, if the reflected wave has one-third the amplitude of the incident wave, the reflection coefficient is 0.33 .

A reflection coefficient of 0.33 is relatively large; usually reflectivity is much lower. Fortunately, the energy reflected is approximately proportional to the square of the reflectivity. In the above example for a reflector with an $\mathrm{RC}$ of 0.33 , only one ninth of the energy is reflected while eight ninths continues the downward journey. So, in most cases, the fraction of energy reflected is minute and, fortunately, almost all the energy is transmitted and available to generate reflections from deeper interfaces.

\section{1.4.3 The Hilbert transform}

The Hilbert transform is a special kind of filter applied to a stacked seismic trace which shifts all positive frequencies of an input signal by -90 degrees and all negative frequencies by +90 degrees, to create a complex trace consisting of a real trace, which is identical to the input stack trace, and an imaginary trace, which is the Hilbert transform of the real, stacked seismic trace.

If $x(t)$ is the input signal, $y(t)$ is the output, and $G(\varpi)$ is the Hilbert transform filter expressed in the frequency domain, then the Hilbert transform process can be expressed mathematically as

$$
x(t) \rightarrow G(\omega)=-j \operatorname{sgn}(\omega) \rightarrow y(t)=H(x(t))
$$


where $H(x(t))$ is the Hilbert transform of $x(t), j=\sqrt{-1}$ and $\operatorname{sgn}(\varpi)$ is defined as:

$$
\operatorname{sgn}(\omega) \begin{cases}=+1 & \omega>0 \\ =-1 & \omega<0 \\ =0 & \omega=0\end{cases}
$$

There are certain requirements on the input signal, $\mathrm{x}(\mathrm{t})$, which are necessary before a valid Hilbert transform can be calculated [65], such as:

1. $\mathrm{x}(\mathrm{t})$ must be real

2. $\mathrm{x}(\mathrm{t})$ can contain no impulses (i.e. true spikes)

3. $x(t)$ can contain no singularities at the origin

A stacked seismic trace satisfies all of these prerequisites so it is a valid candidate for the Hilbert transform process. For a stacked seismic trace, or any valid input x (t), the Hilbert transform, $\mathrm{H}(\mathrm{x}(\mathrm{t}))$, has the following properties:

Property 1: if $\mathrm{x}(\mathrm{t})$ is real then $\mathrm{H}(\mathrm{x}(\mathrm{t}))$ is real

Property 2: $\mathrm{H}($ odd function $)=$ even function

$$
\mathrm{H}(\text { even function })=\text { odd function }
$$

Property 3: $\quad H(H(x(t)))=-x(t)$ i.e. two successive Hilbert transforms yield the negative of the input function 


\section{I.1.4.4 The complex seismic trace}

The concept of the complex seismic trace is illustrated in Figure 13, where $\mathrm{x}(\mathrm{t})$ represents the real seismic trace and $\mathrm{y}(\mathrm{t})$ is the Hilbert transform of $\mathrm{x}(\mathrm{t})$.

The complex trace consists of a real trace, which is identical to the input stack trace, and an imaginary trace, which is the Hilbert transform of the real, stacked seismic trace. Consequently, the Hilbert transform is the key process in the calculation of a complex seismic trace.

The two data vectors $\mathrm{x}(\mathrm{t})$ and $\mathrm{y}(\mathrm{t})$ are displayed in a three-dimensional $(\mathrm{x}, \mathrm{y}, \mathrm{t})$ space in this illustration, where ' $\mathbf{t}$ " is seismic traveltime, ' $\mathbf{x}$ " is the real data plane, and " $y$ " is the imaginary plane. In this complex trace format, the actual seismic trace $x(t)$ is confined to the real $x$-plane, and $y(t)$, the Hilbert transform of $x(t)$, is confined to the imaginary $y$-plane. When $x(t)$ and $y(t)$ are added vectorally, the result is a complex seismic trace $\mathrm{z}(\mathrm{t})$ in the shape of a helical spiral extending along, and centered about, the time axis t. The projection of this complex function $\mathrm{z}(\mathrm{t})$ onto the real plane is the real seismic trace $\mathrm{x}(\mathrm{t})$, and the projection of $\mathrm{z}(\mathrm{t})$ onto the imaginary plane is $y(t)$, the calculated Hilbert transform of $x(t)$.

In the next paragraphs we will present the most important attributes. The commercial software now available does the job of calculating them. Their importance in the process of building a solid and robust relationship between conventional well logs and reservoir characteristics is still unknown and field- dependent.

In the present work 5 seismic attributes were used, they being provided in [13]:

1. Acoustic impedance

2. Conventional amplitudes

3. Instantaneous phase

4. Instantaneous frequency

5. Trace envelope 


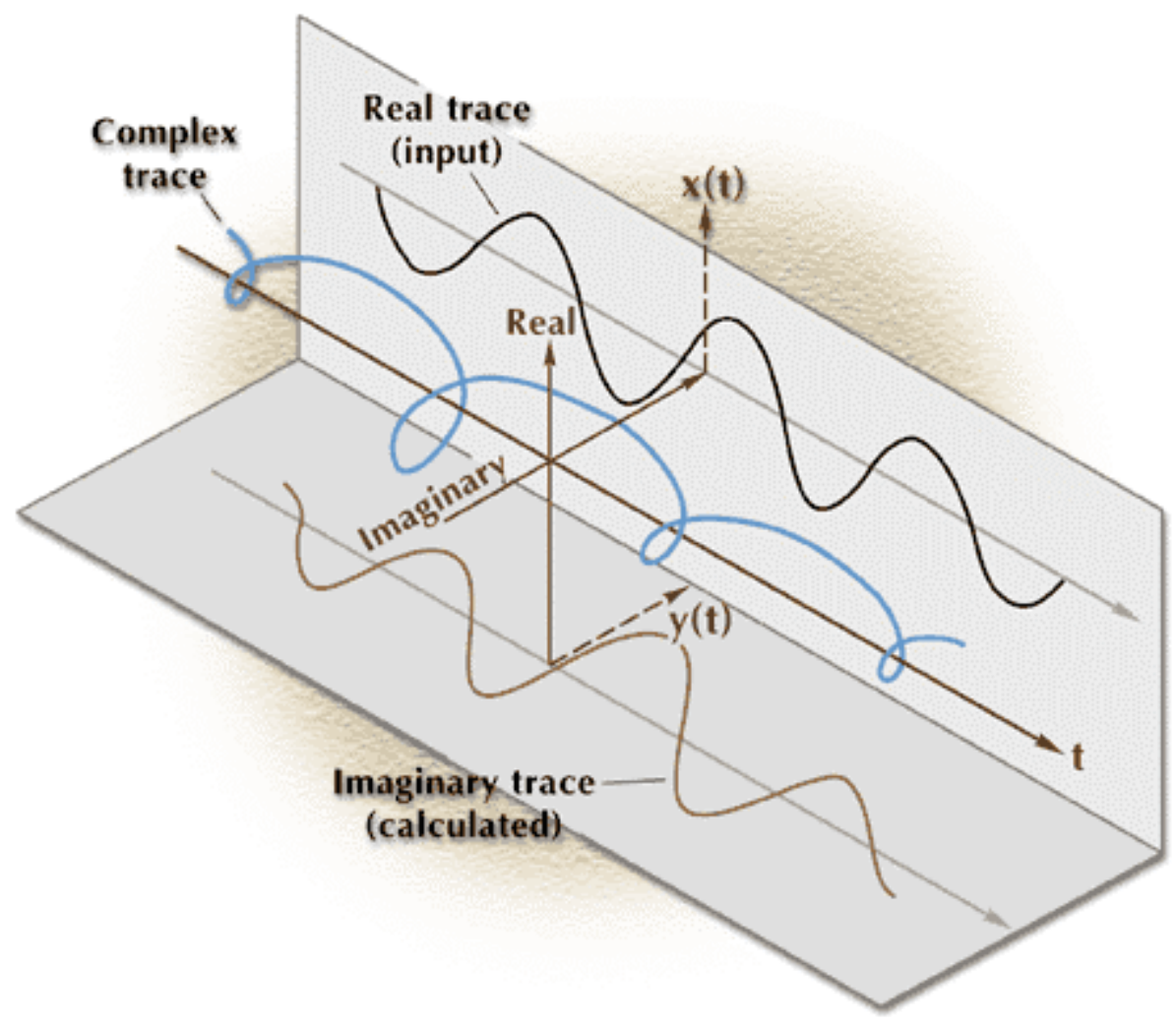

Figure 13 The complex seismic trace [65]

In the Figure 13, the complex seismic trace consists of a real component (actual recorded seismic trace), and an imaginary component which is calculated via the Hilbert Transform. In a 3dimensional plot, the real and imaginary components combine to produce a helical appearing function that spirals about the time axis. 


\section{I.1.4.4.1 The basics of the instantaneous attributes}

The reason for converting the real seismic trace $\mathrm{x}(\mathrm{t})$ into what appears to be a more mysterious complex seismic trace $\mathrm{z}(\mathrm{t})$ is illustrated in Figure 14, where the concepts of instantaneous seismic amplitude, phase, and frequency are introduced. At any point on the time axis, we can calculate a vector a ( $t$ ) that extends away from the $t$ axis in a perpendicular plane to intersect the helically shaped complex seismic trace $\mathrm{z}(\mathrm{t})$.

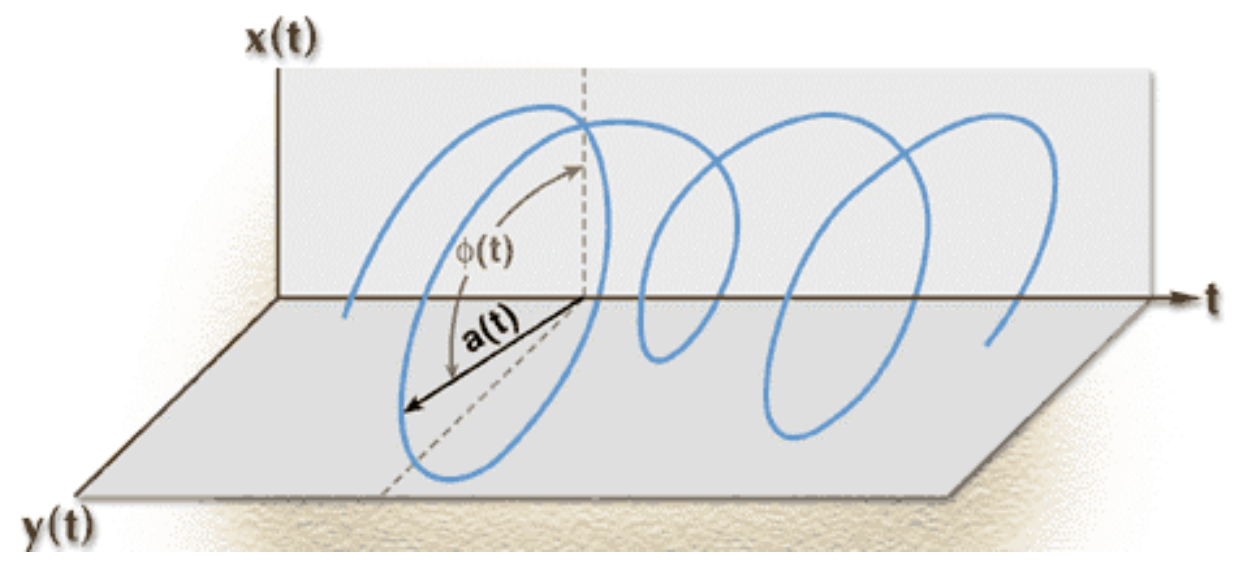

Figure 14 The vectors in the seismic attributes analysis

There were a couple of trials to classify the seismic attributes or to introduce a standard [6], [54] but it seems that the trial was unsuccessful. More than 200 seismic attributes were developed [54] but in [3] they estimate that over 350 seismic attributes can be extracted from a seismic trace. Which are the most important attributes is another question.

In [11] they found that there are three very important attributes- zero phase amplitude, relative impedance and absolute impedance but the literature review (covered in this project) cited that they depend on the field and the reservoir parameter to be predicted- see [47], [48] and [3].

Takahashy et al [53] developed a methodology to select the most important seismic attributes. It is based on the "fuzzy entropy" concept and Shannon's information theory [22]. The methodology involves the fuzzy c-mean clustering. After that the Shannon's system entropy can be computed. 
The seismic attributes that give the smallest values for entropy are the most important seismic attributes. They noted that some attributes are more sensible to particular properties than others.

Anyway the importance of various seismic attributes is field- sensitive. In [7] using the same approach, Chawathe et al [7] found that all the surface seismic attributes that they analyzed for a surface seismic- gamma ray correlation are important: quadrature, reflection strength, instantaneous phase, instantaneous frequency and the trace amplitude.

Balch [3] found the most important seismic attributes using a fuzzy- ranking algorithm. The algorithm statistically determines how well a particular input (seismic attribute) could resolve a particular output (reservoir property at the wellbore) with respect to any number of other inputs using fuzzy curve analysis- the article contains an example about the theory.

In the following, the theory of the most important seismic instantaneous attributes is covered. 


\section{I.1.4.4.2 The trace amplitude}

The instantaneous seismic amplitude is the amplitude of a seismic wiggle trace at a fixed point in time. We mathematically calculate this amplitude value using the equation:

$a(t)=\sqrt{x^{2}(t)+y^{2}(t)}$

There are two important features of instantaneous amplitude [65]:

1. Instantaneous amplitude is not simply the magnitude of the real seismic trace, which is a popular concept for measuring the amplitude of seismic data

2. The maximum of the instantaneous amplitude function does not necessarily occur at the maximum amplitude of the real seismic trace, which surprises some interpreters.

\section{I.1.4.4.3 The instantaneous phase}

The orientation angle $(\mathrm{t})$ of the amplitude vector a $(\mathrm{t})$ at time $\mathrm{t}$, which is generally measured relative to the positive axis of the real $\mathrm{x}$-plane, is defined as the phase of $\mathrm{z}(\mathrm{t})$ at that moment in time: hence the term instantaneous phase. Numerically, the phase angle is calculated from the equation:

$\phi(t)=\tan ^{-1}\left(\frac{y(t)}{x(t)}\right)$

As seismic time progresses, vector a $(t)$ not only moves along the $t$ axis, but it also rotates continually about the time axis to maintain contact with the spiraling complex trace $\mathrm{z}(\mathrm{t})$. Each full rotation of the vector about the time axis increases the phase value by 360 degrees. 


\section{I.1.4.4.4 The instantaneous frequency}

The instantaneous frequency is the frequency of an oscillating function at a fixed point in time. In any oscillating system and specifically for a seismic trace that oscillates between positive values (peaks) and negative values (troughs), frequency can be defined as the time rate of change of the phase angle:

$$
\omega(t)=\frac{d}{d t}[\phi(t)]
$$

\section{I.1.4.4.5 The trace envelope}

The instantaneous amplitude is a positive definite function, meaning that its numerical value is always a positive number. It is plotted as both a positive and a negative function to emphasize the concept that it is the envelope of both the real and imaginary parts of the complex trace.

The trace envelope (as well as instantaneous phase and frequency, discussed below) is derived from the complex trace. The real part of the complex trace is the trace itself; the imaginary part is its Hilbert Transform, which is basically a 90-degree phase shift of the trace.

The trace envelope is defined as the magnitude of the complex trace, and it is useful for obtaining a running average of the absolute reflection strength- Figure 15.

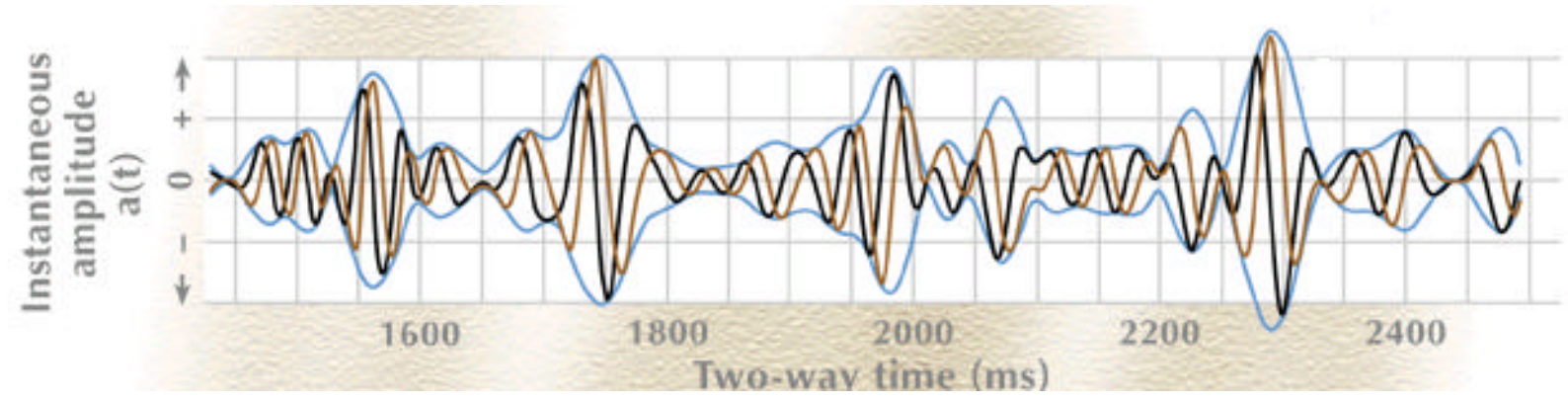

Figure 15 The instantaneous-amplitude function calculated from a complex seismic trace 


\section{I.2 THE CROSSWELL SEI SMI C}

\section{I.2.1 Description and comparison with other industrial applications}

Traditionally, seismic data have been gathered by surface sources and receivers. The next logical step in the effort to improve seismic resolution is to place both source and geophone in the subsurface.

During the last past years, geophysicists have applied seismic tomography to imagine velocity variations of the earth.

Seismic crosswell tomography is a method of determining seismic velocity (wave speed) between boreholes. The tomographic image has a resolution of $10 \div 25$ feet and can supply otherwise unavailable information on geological and reservoir characterization parameters.

Seismic tomography is similar in principle to medical tomographic methods, but several factors conspire to make seismic tomography more difficult than the medical case. This is illustrated in the comparison below:

\section{Medical X-ray Tomography:}

$\checkmark$ Imaged object can be completely surrounded by sources and receivers (full aperture).

$\checkmark$ Image rays travel at constant velocity and are straight.

$\checkmark$ Uniform ray coverage.

$\checkmark$ Resolution dictated by X-ray wavelength on the order of Angstroms. 


\section{Seismic Tomography:}

$\checkmark$ Imaged object cannot be completely surrounded by sources and receivers (limited aperture).

$\checkmark$ Image rays travel at variable velocity (sound speed in rocks) and are bent.

$\checkmark$ Irregular ray coverage due to ray bending and limited recording aperture.

$\checkmark$ Resolution dictated by seismic wavelength on the order of $10 \div 25 \mathrm{ft}$.

Usually the receivers remain fixed at their given locations while the source move upward after each shot- Figure 16.
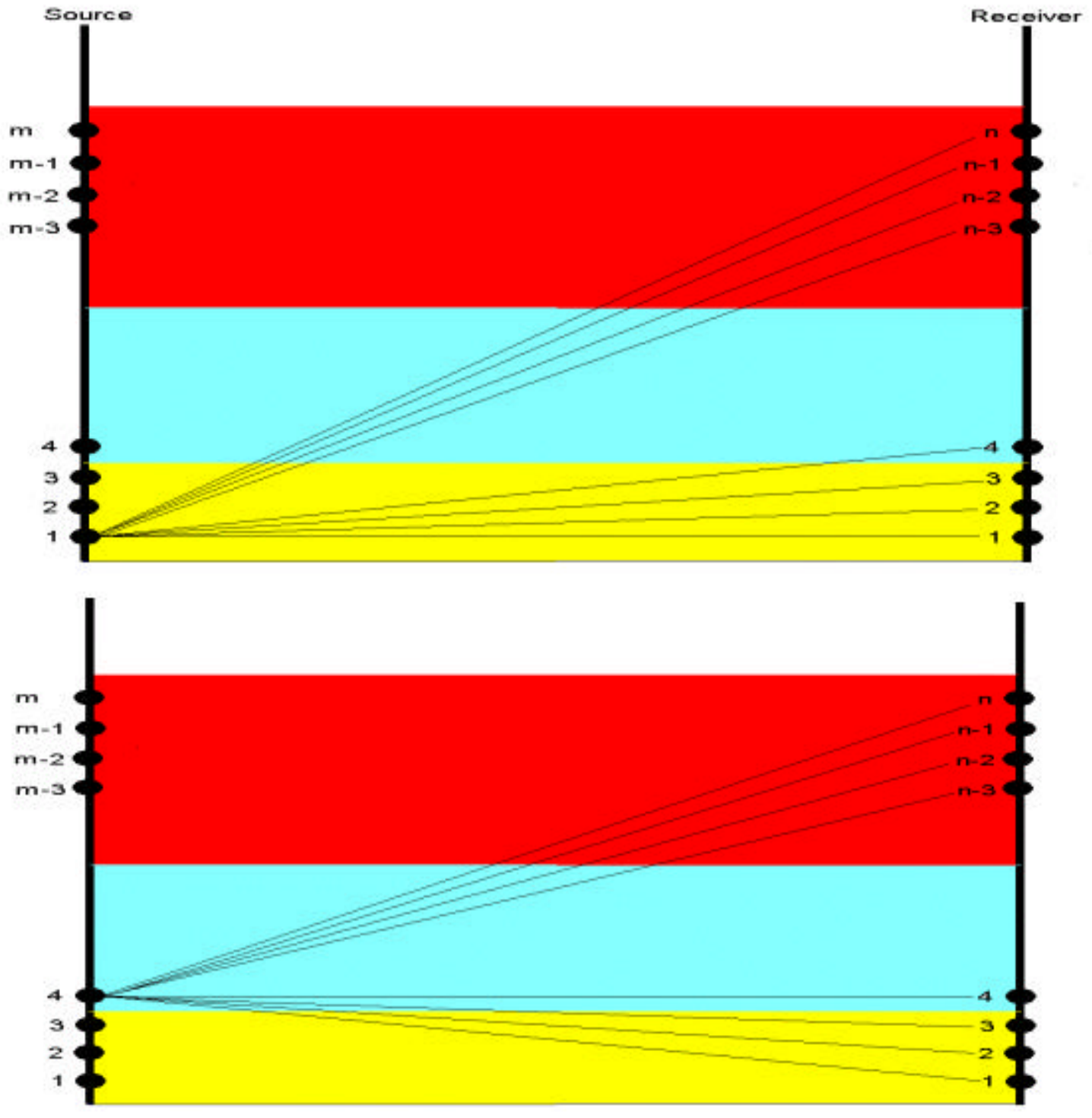

Figure 16 The crosswell seismic data acquisition 
The information obtained from crosswell seismic experiments consist of:

- Travel times

- Seismic reflections

The Figure 17 contains the sketch of the crosswell seismic ray coverage. The rays better cover some areas (in the central zone) than other areas (some of them are not intersected by the rays).

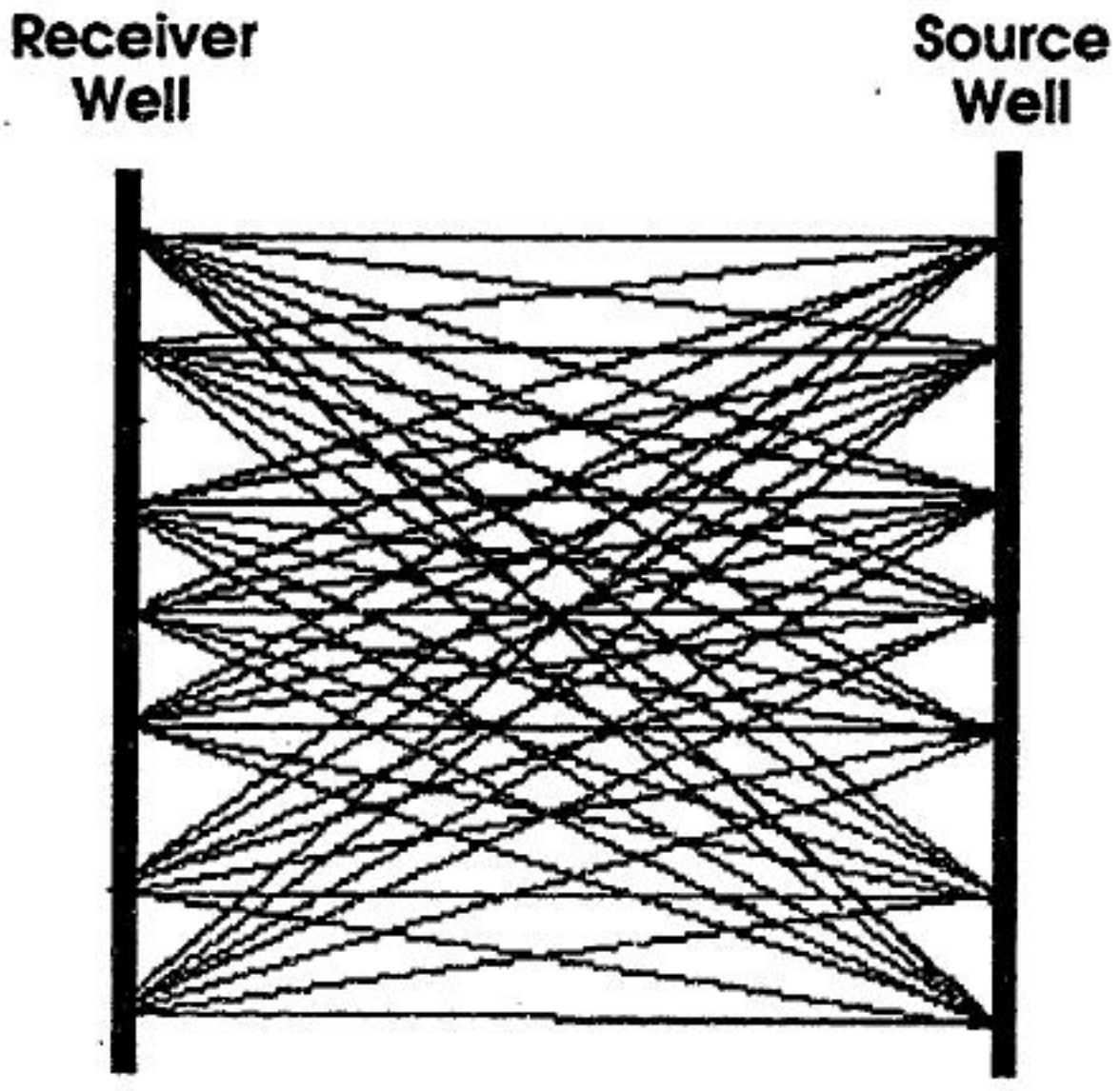

Figure 17 The crosswell seismic ray coverage 


\subsubsection{The traveltime inversion problem}

\section{I.2.2.1 The theory of the traveltime inversion}

Traveltime tomography (tomo=slice+graph=picture) is the procedure for reconstructing the earth's velocity model from seismic data (from picked traveltimes). These traveltimes can be measured from seismic data associated with a variety of source-receiver configurations, and can be used to extract velocity models of varying spatial resolution.

There are two main types of seismic data to be inverted: traveltime data and waveform data. Traveltime tomography reconstructs earth velocity models with several times lower resolution compared to waveform tomograms. But on the other hand traveltime tomography is typically much more robust, easier to implement, and computationally much cheaper.

The main topic is seismic traveltime inversion in 2- and 3- dimensional heterogeneous media. A typical problem is to infer the compressional- wave slowness (reciprocical of velocity) distribution of a medium, given a set of observed first- arrival traveltimes between the sources and receivers of known location within the medium.

This problem is common for crosswell seismic transmission tomography imaging in a 2-D region between vertical boreholes in oil-field applications.

When a sound wave or seismic wave is launched into a medium, it takes time for the influence of the wave to progress from the source to a more distant point. This time is called traveltime. We also define a local wave speed associated with each point in the medium by considering the average wave speed for two points that are very closely spaced. 
The local slowness is the inverse of the local wave speed. It is most convenient to develop inversion and tomography formulas in terms of wave slowness models, because the pertinent equations are linear in slowness [4]. However, we often make one of two more restrictive assumptions that:

1. The model comprises heterogeneous cells (in 2-D) or blocks (in 3-D) with $\mathrm{s}_{\mathrm{j}}$ denoting the slowness value of the $\mathrm{j}^{\text {th }}$ cell or block

2. The model is composed of a grid with values of slowness assigned at the grid point together with some interpolation scheme (bilinear, spline...)

In linear inversion with block models, we must solve a linear system of equations. In this chapter $\mathbf{m}$ is the number of the available traveltimes and $\mathbf{n}$ is the number of the cells where the slowness in unknown. There are usually many more equations than unknowns.

The major difficulties in solving the system of equations are:

1. the matrix is not a square

2. the matrix is often rank deficient

3. the matrix is often poorly conditioned

Classical traveltime tomography uses raypaths to model traveltimes and so assumes that the data are of high frequency, i.e., the wavelength is at least several times smaller than the spatial variations of the velocity model. In this case, the traveltime measurements are connected to the model via the traveltime integral [49]:

$t_{(x, y)}=\int_{\text {raypath }} \frac{1}{c(x, z)} d s$

The traveltime integral says that the traveltime for energy to propagate from source to receiver is computed by integrating the weighted slowness $\mathrm{s}(\mathrm{x}, \mathrm{z})=1 / \mathrm{c}(\mathrm{x}, \mathrm{z})$ along the raypath that connects the source and receiver. 
There are several steps to inverting traveltime data:

1. Pick the traveltime $t_{i}$ from the $i^{\text {th }}$ raw record, and construct the $m x 1$ traveltime vector comprised of $m$ traveltime picks.

2. Suppose that the earth model is composed by $\mathbf{n}$ slowness cells, where each $i^{\text {th }}$ cell has an unknown constant slowness $\mathbf{s}_{\mathbf{i}}$.

3. Denote the segment length of the $\mathrm{i}^{\text {th }}$ ray in the $\mathrm{j}^{\text {th }}$ cell by $\mathrm{l}_{\mathrm{ij}}$. In this case the traveltime integral reduces to a summation of weighted slowness, where the weight is the segment length of the rays:

$t_{i}=\sum_{j} l_{i j} s_{j}$

4. For some initial guess slowness model "s" we have $t=L s$ which can be used to form the perturbed set of traveltime equations:

$t-t^{\prime}=L s-L^{\prime} s^{\prime}$

where $\mathbf{L}$ is the raypath matrix associated with the guessed $\mathbf{s}$ model.

The above equation becomes:

$\mathrm{Dt}=\mathrm{Lds}$

This is known as the linearization step where the relationship between the model and data is linearized by equation $\mathrm{Dt}=\mathrm{Lds}$; this implicitly assumes that the segment lengths do not change when the slowness model is slightly perturbed from the actual slowness model. The equation can be solved by minimizing the sum of the squared errors. 


\section{I.2.2.2 The nonuniqueness problem, constraints}

Cross-borehole seismic surveys have important advantages over surface seismic. The crosshole energy avoids the filtering and attenuation effects associated with a weathered surface layer; sources and receivers may be placed closer to the region of interest; and shorter travel paths allow reception of higher frequencies, providing higher resolution [20]. However, these advantages are partially offset by some serious disadvantages.

The most critical problem in crosshole tomographic imaging is the mathematical nonuniqueness of the solutions obtained. The limited range of viewing angles in a crosshole survey results in singular matrices in the inversion, because the traveltime equations are not all linearly independent.

The system is thus under- determined even when the number of observations is much greater than the number of unknowns, and many solutions can be found that fit the data equally well. This nonuniqueness is inherent in the crosshole geometry and affects both direct inversion and iterative methods. However, in many practical applications incomplete angular coverage, and the attendant nonuniqueness problem, is unavoidable.

There are alternative strategies for reducing nonuniqueness. Limits may be imposed $\mathrm{o}$ the statistical properties of the solution; for example, a condition of minimum solution variance may be imposed, resulting in the smoothest possible reconstruction compatible with the data. The approach followed by the USBM in GEOTOM3D is to supplement the incomplete traveltime data set with additional site information. This additional information can be incorporated into the inversion to a certain extent through the use of an appropriate starting model, and more explicitly in the form of constraints limiting the range of permissible solutions. 
Commonly available supplementary information includes borehole logs of sonic velocities and lithology, surface refraction or reflection data, and local or regional structural and stratigraphic information. In many cases such information permits definition of the types of solutions that would be considered geologically "reasonable" among the multitude of mathematically permissible solutions.

Iterative reconstruction methods begin by specifying a "reasonable" initial model, and in general they remain close to the starting model, except where modifications are required to fit the data. In addition, however, certain explicit constraints can be incorporated that are not possible with direct matrix inversion methods. For example, the solution can be forced to match known boundary values (e.g., known borehole velocities); the solution range may be bounded (i.e., maximum and/or minimum allowable velocities may be specified); and the solution may be damped selectively (e.g., layer uniformity may be maintained by averaging over groups of pixels). Iterative methods thus allow greater range and flexibility in the nature of constraints that may be applied.

All the USBM software (including MIGRATOM, GEOTOM3D) solve the tomography using the simultaneous iterative reconstruction technique (SIRT), which repeatedly modifies an initial model to obtain the best possible fit to the data.

SIRT calculations modify an arbitrary initial velocity model by repeated cycles of three steps: forward computation of model travel times, calculation of residuals, and application of velocity corrections. The cycle repeats through a number of iterations until the termination criteria were fulfilled.

The SIRT methodology is covered in all of the USBM reports listed above ([20], [56], [21], [57]). Also, a good presentation about the traveltime tomography theory (including SIRT method) was made in [49] by Gerald Schuster. 


\subsubsection{The complexity of the raypaths in the crosswell seismic experiments}

Crosswell seismic data can contain a variety of wave modes even in the simplest geologic setting. This occurs because crosswell seismic propagation is predominantly parallel to geologic bedding rather than perpendicular to it as in surface seismic recording. A common shot gather across a single elastic bed boundary can generate eight distinct body wave arrivals for a source generating both $\mathrm{P}$ and $\mathrm{S}$ waves [18]- Figure 18. These arrivals consist of direct $\mathrm{P}$ and $\mathrm{S}$ waves, reflected $\mathrm{P}$ and $\mathrm{S}$ waves, $\mathrm{P}-\mathrm{S}$ and S-P reflected-converted waves, $\mathrm{P}-\mathrm{S}$ and S-P transmitted-converted waves. The figures are:

(a) direct $\mathrm{P}$ or $\mathrm{S}$ wave raypaths

(b) S-P transmitted- conversion raypaths

(c) P-S transmitted- conversion raypaths

(d) P or S- wave reflection raypaths

(e) P-S reflected- conversion raypaths

(f) S-P reflected- conversion raypaths

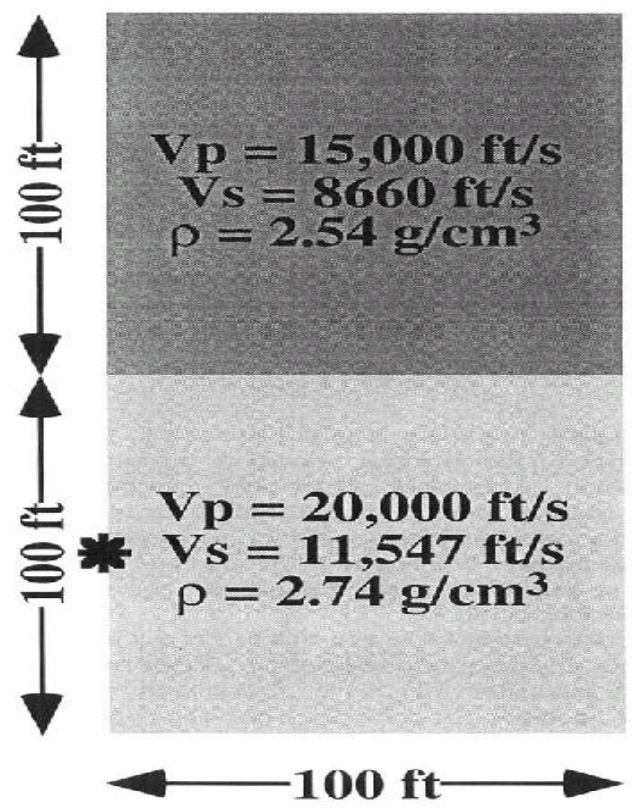

(a)

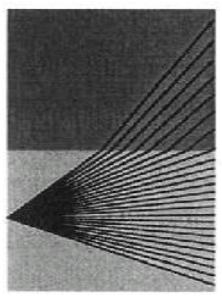

(d)

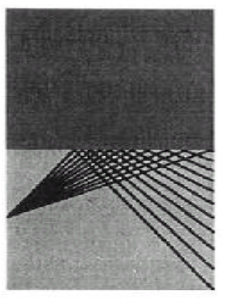

(b)

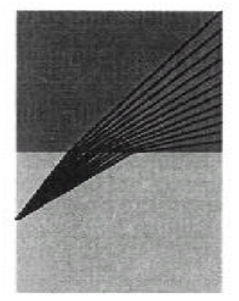

(e)

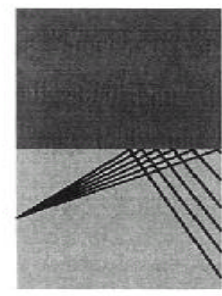

(c)

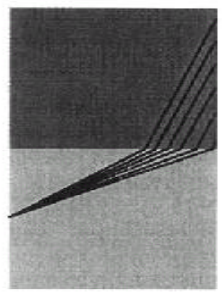

(f)

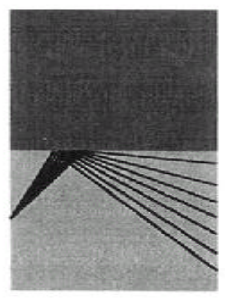

Figure 18 A simple single- interface model to illustrate some of the many seismic models generated in a typical crosswell experiment. 
Figure 19 provides an illustration of the moveout of these various modes in the space-time display of a common shot gather [18]. The traveltimes are calculated using Snell's law.

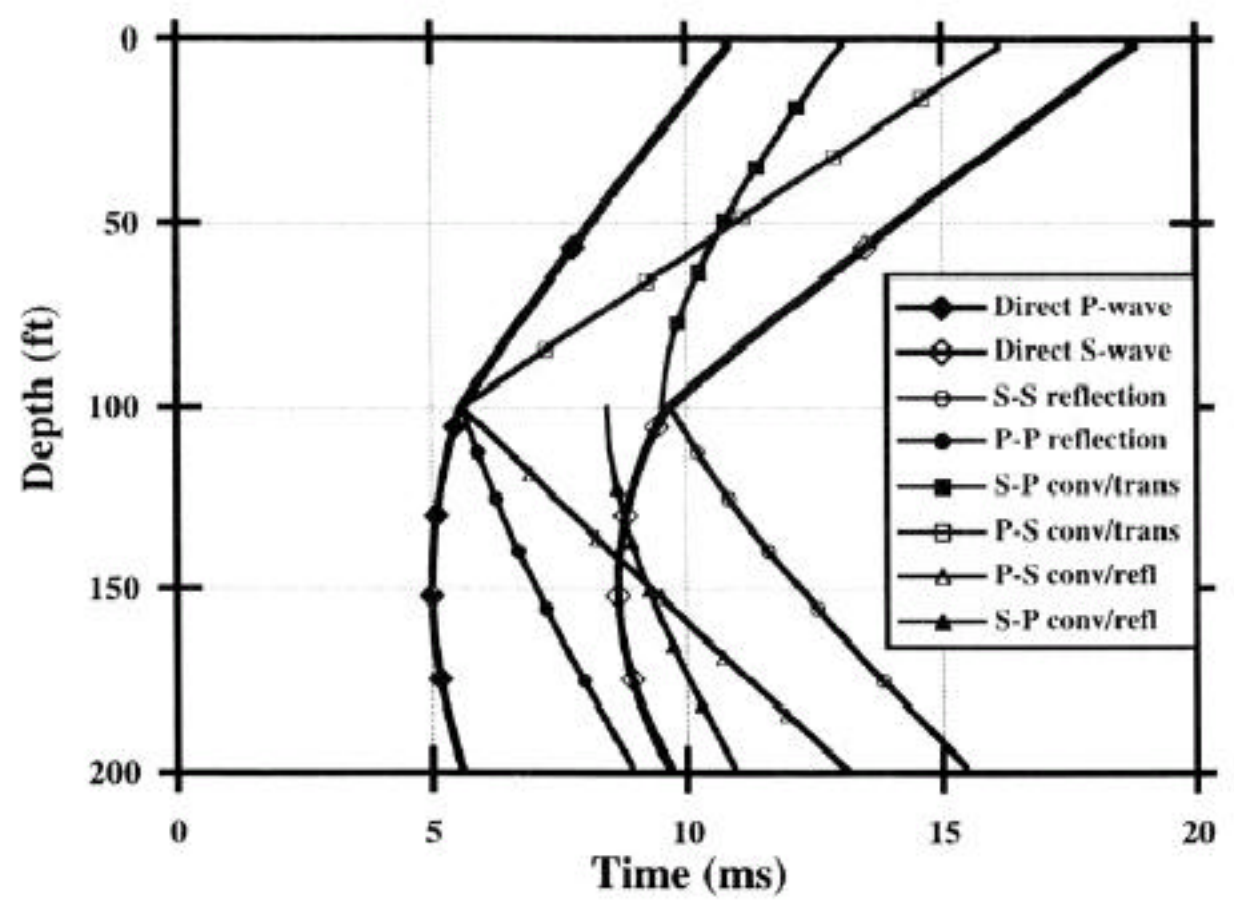

Figure 19 A space-time record for a simple single-interface model shown in Figure 18 [18]

Due to the Snell's law, the raypaths become curved because the layers usually consist of many interfaces between zones of different acoustic impedances. For a zone analyzed in [27], the raypaths are depicted in the Figure 20. 


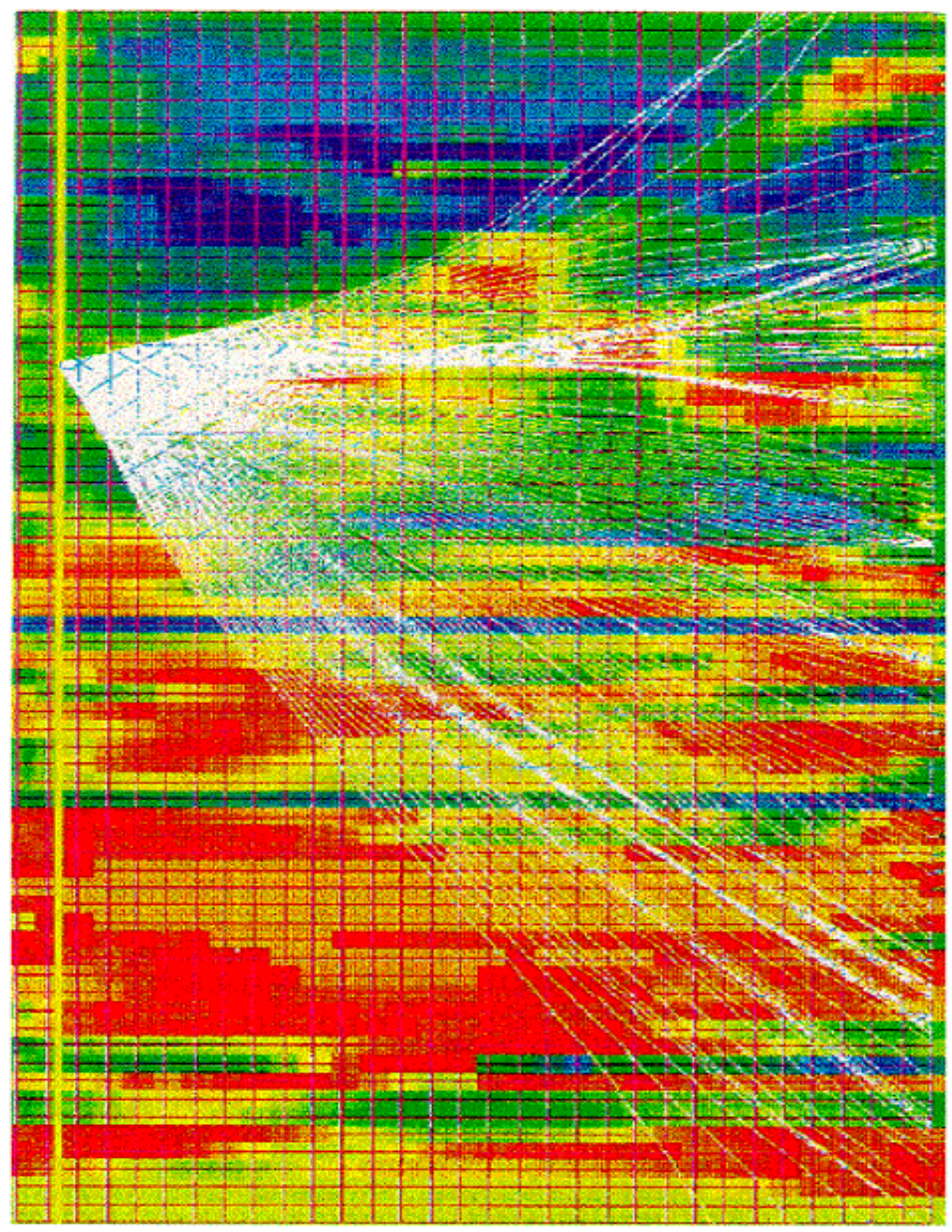

Figure 20 Curved ray tracing for a field [27] 
Another research shown the problem to be even more complicated [40]. Whatever the level of sophistication, crosshole tomography crucially depends on the arrival time information. Inherent in this procedure are two fundamentals assumptions:

1. The first arrivals as recorded along the receiver borehole reflect the influence of variations in velocity

2. The propagation of energy is confined to the plane common to the source and receiver boreholes and it follows straight paths except at points of changes in velocity where it undergoes refractive bending in accordance with Snell's law.

Mufti [40] noticed that in crosswell seismic the target of interest can be a finite object with geometric features comparable in size to the dominant wavelength of the source signal. Under these conditions, a significant amount of energy, can bypass the object under investigation as a result of diffraction. When the velocity of the anomalous body is less than that of the surrounding medium, the bypassed energy can appear as the first arrival in the receiver borehole in accordance with Fermat's principle.

Under the previous listed assumptions he found that the first arrivals recorded along the receiver borehole represent the energy which is either transmitted through the different velocity zone or bypasses it completely.

For different source locations, the differences in the arrival times of direct and bypassed events are very sensible to the 3-D shape of a velocity anomaly. He discovered that, unless the two surfaces at which the energy enters and leaves the reservoir are parallel, a 2D inversion algorithm, whatever its level of sophistication and accuracy, will invariably lead to incorrect results. 


\section{CHAPTER II - ARTIFICIAL NEURAL NETWORKS}

\section{I.1 DESCRI PTI ON AND SI MI LARITIES WITH THE HUMAN LOGIC}

Neural networks are universal function estimators. With their remarkable ability to derive meaning from complicated or imprecise data, they can be used to extract patterns and detect trends that are too complex to be noticed by either humans or other computer techniques.

Artificial neural networks (ANNs) are computational paradigms which implement simplified models of their biological counterparts, biological neural networks. Biological neural networks are the local assemblages of neurons and their dendritic connections that form the (human) brain. Accordingly, ANNs are characterized by:

- Local processing in artificial neurons (or processing elements, PEs)

- Massively parallel processing, implemented by rich connection pattern between PEs

- The ability to acquire knowledge via learning from experience

- Knowledge storage in distributed memory, the synaptic PE connections

The basic processing element in the nervous system is the neuron. The human brain is composed of about 10 to 500 billion neurons. Tree-like networks of nerve fiber called dendrites are connected to the cell body or soma, where the cell nucleus is located. Extending from the cell body is a single long fiber called the axon, which eventually branches into strands and substrands, and are connected to other neurons through synaptic junctions, or synapses. One simple neuron cell is represented in Figure 21 [64]

The neurons are divided into modules and each module contains about 500 neural networks [33]. Each network may contain about 100,000 neurons in which each neuron is connected to hundreds to thousands of other neurons. This architecture is the main driving force behind the complex behavior that comes so natural to us. 
The transmission of signals from one neuron to another, at a synapses, is a complex chemical process in which specific transmitter substances are released from the sending end of the junction. The effect is to raise the electrical potential inside the body of the receiving cell. If the potential reaches a threshold, a pulse is sent down the axon - we then say the cell has "fired".

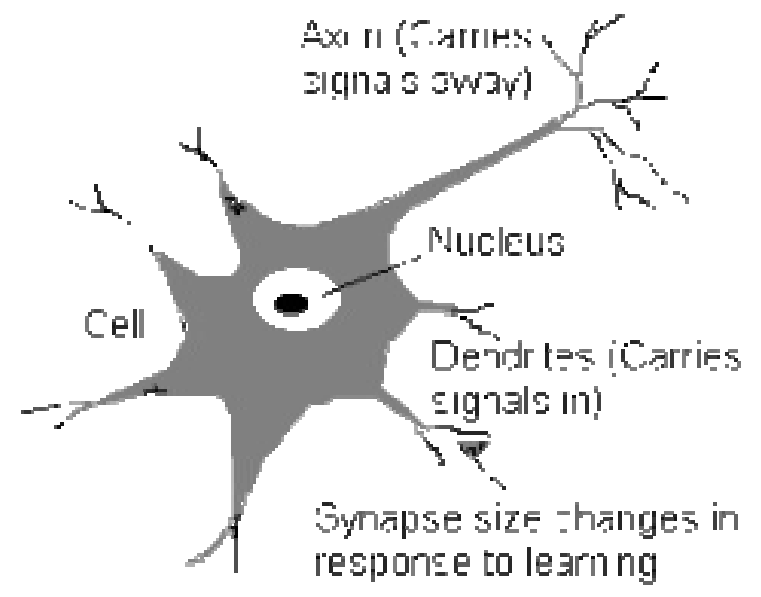

Figure 21 A simple neuron cell

Artificial neural networks have been developed as generalization of mathematical models of human cognition or neural biology, based on the assumptions that:

1. Information processing occurs in many simple elements that are called neurons (processing elements)

2. Signals are passed between neurons over connections links

3. Each connection link has an associated weight, which, in a typical neural network, multiplies the signal being transmitted

4. Each neuron applies an activation function (usually non-linear) to its net input to determine its output signal

In a simplified mathematical model of the neuron (Figure 22), the effects of the synapses are represented by "weights" (w) which modulates the effect of the associated input signals (x), and the nonlinear characteristics exhibited by neurons is represented by a transfer function which is 
usually the sigmoid function. The neuron impulse is then computed as the weighted sum of the input signals, transformed by the transfer function.

The learning capability of an artificial neuron is achieved by adjusting the weights in accordance with the chosen learning algorithm.

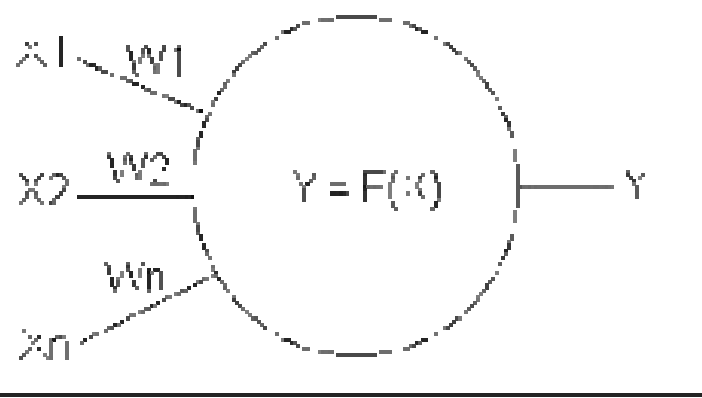

\section{Figure 22 A schematic diagram of a neuron}

In a typical neural data processing procedure, the database is divided into three separate portions called training, calibration and verification sets. They are chosen randomly from the entire dataset; the goal is to predict as well as possible the verification set.

The training set is used to develop the desired network. In this process (depending on the paradigm that is being used), the desired output in the training set is used to help the network adjust the weights between its neurons or processing elements.

During the training process the question arises as when to stop the training because a network can be overtrained. Once the network memorizes a data set, it would be incapable of generalization. In order to avoid overtraining it is a common practice to stop the training process so often and apply the network to the calibration data set.

Once the training process is completed successfully, the network is applied to the verification data set. 


\section{I .2 NEURAL NETWORKS VERSUS CONVENTI ONAL COMPUTERS}

Neural networks take a different approach to problem solving than conventional algorithms. Conventional algorithms follow a set of instructions in order to solve a problem [52]. Unless the specific steps that computer needs to follow are known the computer cannot solve the problem. That restricts the problem solving capability of conventional algorithms to problems that we already understand and know how to solve. But computers would be so much more useful if they could do things that we don't exactly know how to do.

Neural networks process information in a similar way the human brain does. The network is composed of a large number of highly interconnected processing elements (neurons) working in parallel to solve a specific problem. Neural networks learn by example. They cannot be programmed to perform a specific task. The examples must be selected carefully otherwise useful time is wasted or even worse the network might be functioning incorrectly. There is one disadvantage- because the network finds out how to solve the problem by itself; its operation can be unpredictable.

On the other hand, conventional computers use a cognitive approach to problem solving; the way the problem is solved must be known and stated in unambiguous instructions. These instructions are then converted to a high level language program and then into machine code that the computer can understand. These machines are totally predictable; if anything goes wrong the software will crash.

Neural networks and conventional algorithmic computers are not in competition but complement each other. There are tasks are more suited to an algorithmic approach like arithmetic operations and tasks that are more suited to neural networks. Even more, a large number of tasks require systems that use a combination of the two approaches (normally a conventional computer is used to supervise the neural network) in order to perform at maximum efficiency.

Other differences can be [10]: 


\begin{tabular}{|c|c|}
\hline Digital Computers & Neural Networks \\
\hline $\begin{array}{l}\text { Deductive Reasoning. We apply known rules to } \\
\text { input data to produce output. }\end{array}$ & $\begin{array}{l}\text { Inductive Reasoning. Given input and output data } \\
\text { (training examples), we construct the rules. }\end{array}$ \\
\hline $\begin{array}{c}\text { Computation is centralized, synchronous, and } \\
\text { serial. }\end{array}$ & $\begin{array}{c}\text { Computation is collective, asynchronous, and } \\
\text { parallel. }\end{array}$ \\
\hline $\begin{array}{c}\text { Memory is packetted, literally stored, and location } \\
\text { addressable. }\end{array}$ & $\begin{array}{c}\text { Memory is distributed, internalized, and content } \\
\text { addressable. }\end{array}$ \\
\hline $\begin{array}{l}\text { Not fault tolerant. One transistor goes and it no } \\
\text { longer works. }\end{array}$ & $\begin{array}{l}\text { Fault tolerant, redundancy, and sharing of } \\
\text { responsibilities. }\end{array}$ \\
\hline Fast. Measured in millionths of a second. & Slow. Measured in thousandths of a second. \\
\hline Exact. & Inexact. \\
\hline Static connectivity. & Dynamic connectivity. \\
\hline $\begin{array}{c}\text { Applicable if well defined rules with precise input } \\
\text { data. }\end{array}$ & $\begin{array}{l}\text { Applicable if rules are unknown or complicated, or } \\
\text { if data is noisy or partial. }\end{array}$ \\
\hline
\end{tabular}




\section{I .3 GENERAL REGRESSI ON NEURAL NETWORK}

General Regression Neural Networks (GRNN) are memory-based feed forward networks based on the estimation of probability density functions. GRNNs feature fast training times and can model non-linear functions. Originally developed in the statistics literature and known as NadarayaWatson kernel regression, GRNN was 're-discovered' by Donald Specht in 1990 [51].

GRNN or "General Regression Neural Network" is Donald Specht's term for a Neural Network invented by him. You can think of it as a normalized RBF (adial $\underline{\text { Basis }}$ Functions) network in which there is a hidden unit centered at every training case. These RBF units are usually probability density functions such as the Gaussian. The only weights that need to be learned are the widths of the RBF units. These widths (often a single width is used) are called "smoothing parameters" or "bandwidths".

GRNN is a universal approximator for smooth functions, so it should be able to solve any smooth function-approximation problem given enough data. The main drawback of GRNN is that it suffers badly from the curse of dimensionality. GRNN cannot ignore irrelevant inputs without major modifications to the basic algorithm. So GRNN is not likely to be the top choice if you have more than 5 or 6 no redundant inputs.

The regression of a dependent variable, $\mathbf{Y}$, on an independent variable, $\mathbf{X}$, is the computation of the most probable value of $\mathrm{Y}$ for each value of $\mathrm{X}$ based on a finite number of possibly noisy measurements of $\mathrm{X}$ and the associated values of $\mathrm{Y}$. The variables $\mathrm{X}$ and $\mathrm{Y}$ are usually vectors.

In order to implement system identification, it is usually necessary to assume some functional form.

In the case of linear regression, for example, the output $\mathrm{Y}$ is assumed to be a linear function of the input, and the unknown parameters, $\mathrm{a}_{\mathrm{i}}$, are linear coefficients 
The procedure presented in Donald F. Specht's article [51] frees it from the necessity of assuming a specific functional form

A Euclidean distance is calculated between an input vector and these weights, which are then rescaled by the spreading factor. The radial basis output is then the exponential of the negatively weighted distance having the form [17], [67] and [8]:

$$
D_{i}^{2}=\left(X-X_{i}\right)^{T}\left(X-X_{i}\right)
$$

The GRNN equation is:

$$
Y(X)=\frac{\sum_{i=1}^{n} Y_{i} \exp \left(-\frac{D_{i}^{2}}{2 \sigma^{2}}\right)}{\sum_{i=1}^{n} \exp \left(-\frac{D_{i}^{2}}{2 \sigma^{2}}\right)}
$$

The estimate $\mathrm{Y}(\mathrm{X})$ can be visualized as a weighted average of all of the observed values, $\mathrm{Y}_{\mathrm{i}}$, where each observed value is weighted exponentially according to its Euclidian distance from X. $\mathrm{Y}(\mathrm{X})$ is simply the sum of Gaussian distributions centered at each training sample. However the sum is not limited to being Gaussian.

In the above examples $\mathrm{X}$ is the case to be predicted. In the present project it is a vector consisting of 8 elements (inputs), where the output "Y" will be predicted.

In this theory, $\sigma$ is the smoothing factor and can be seen as the spread of the Gaussian bell.

A small value of the smoothing factor - Figure 23 caused the estimated parent density function to have distinct modes corresponding to the locations of the training samples [9]. The Figure 23 can be seen as a case where the output (one element) is predicted based on two inputs. 


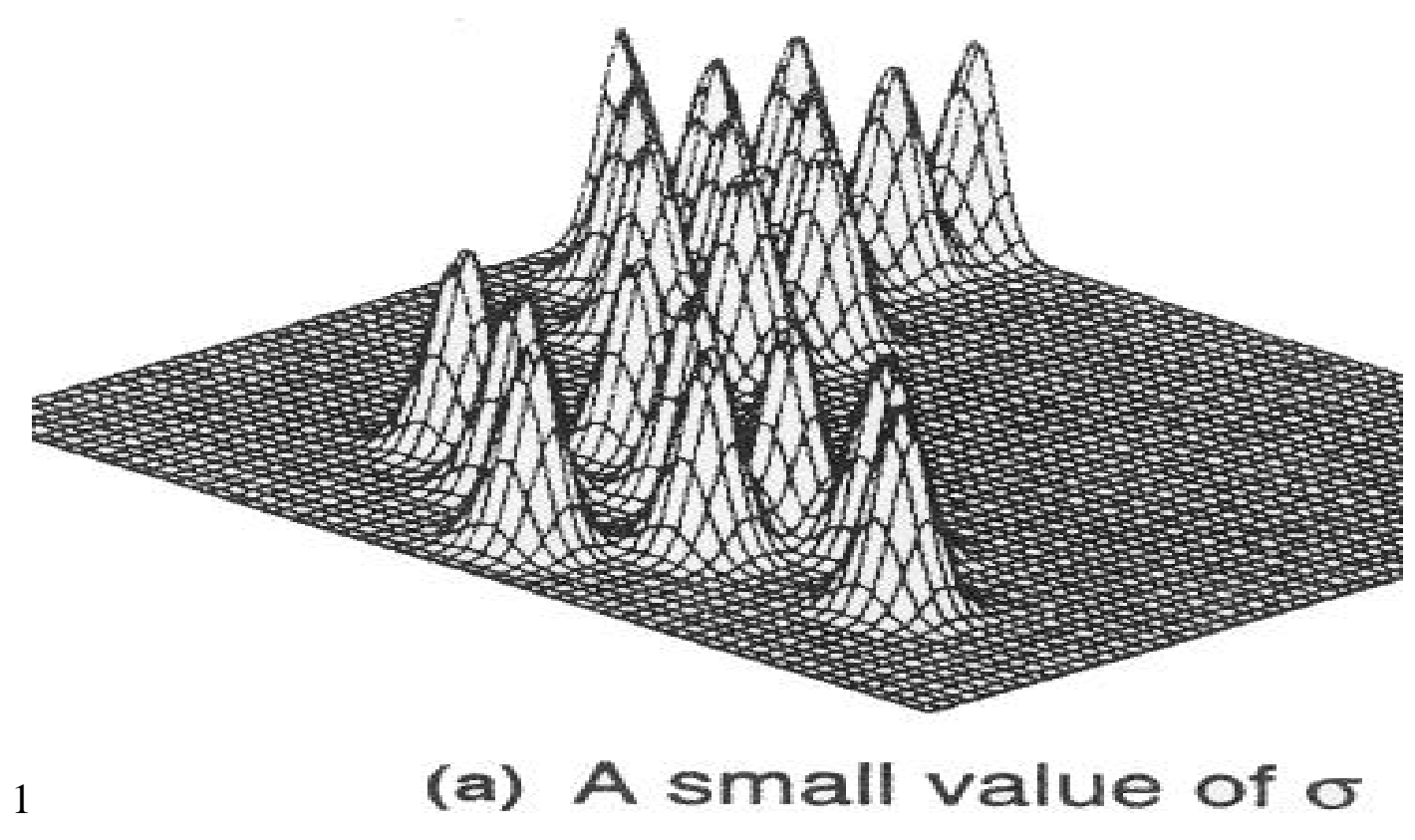

Figure 23 The estimated parent density function for a small value of the smoothing factor

A larger value of the smoothing factor - Figure 24 produces a greater degree of interpolation between points.

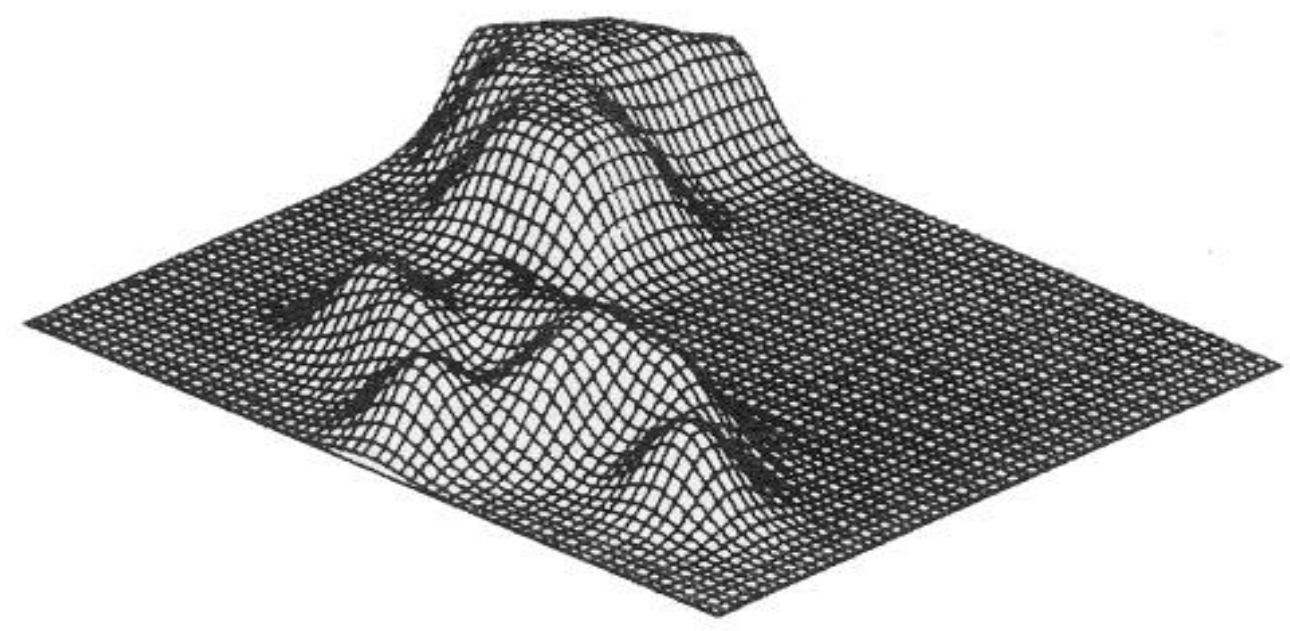

(b) A larger value of $\sigma$

Figure 24 The estimated parent density function for an intermediate value of the smoothing factor 
A very large value of the smoothing factor- Figure 25 would cause the estimated density to be Gaussian regardless of the true underlying distribution

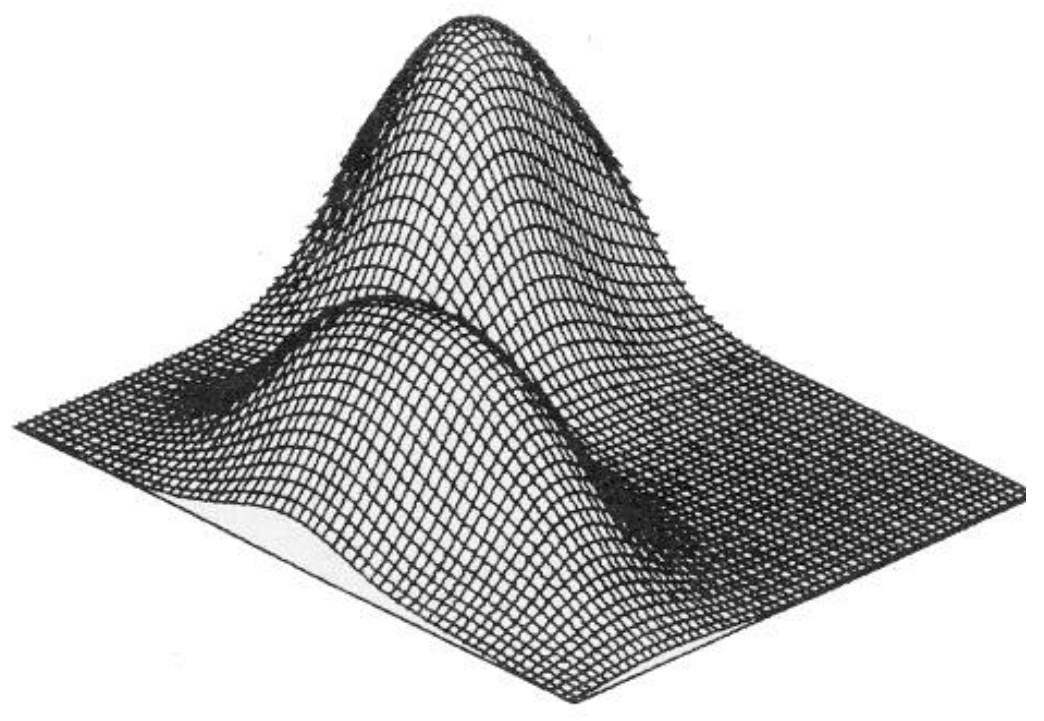

(c) An even larger value of $\sigma$

Figure 25 The estimated parent density function for a larger value of the smoothing factor 


\section{I .4 NEURAL NETWORKS APPLI CATI ONS I N THE PETROLEUM I NDUSTRY}

The last decade of the $20^{\text {th }}$ century brought into the petroleum industry a new tool- the Artificial Neural Networks. Neural networks have shown great potential for generating accurate analysis and results from large historical databases.

In 1994 Mohaghegh, Ameri et al were able to predict the permeability using as inputs depth, gamma ray, bulk density, deep induction log and zonal subdivision specification. The zone identification were also possible using the log data and the slopes of the log plot [62]. The data came from Granny Creek Field, West Virginia.

The Artificial Intelligence methods were proved to be superior comparing to simple regression models or empirical models [37] and [38]. Using the logs Mohaghegh et al were able to predict permeability- Figure 26. 


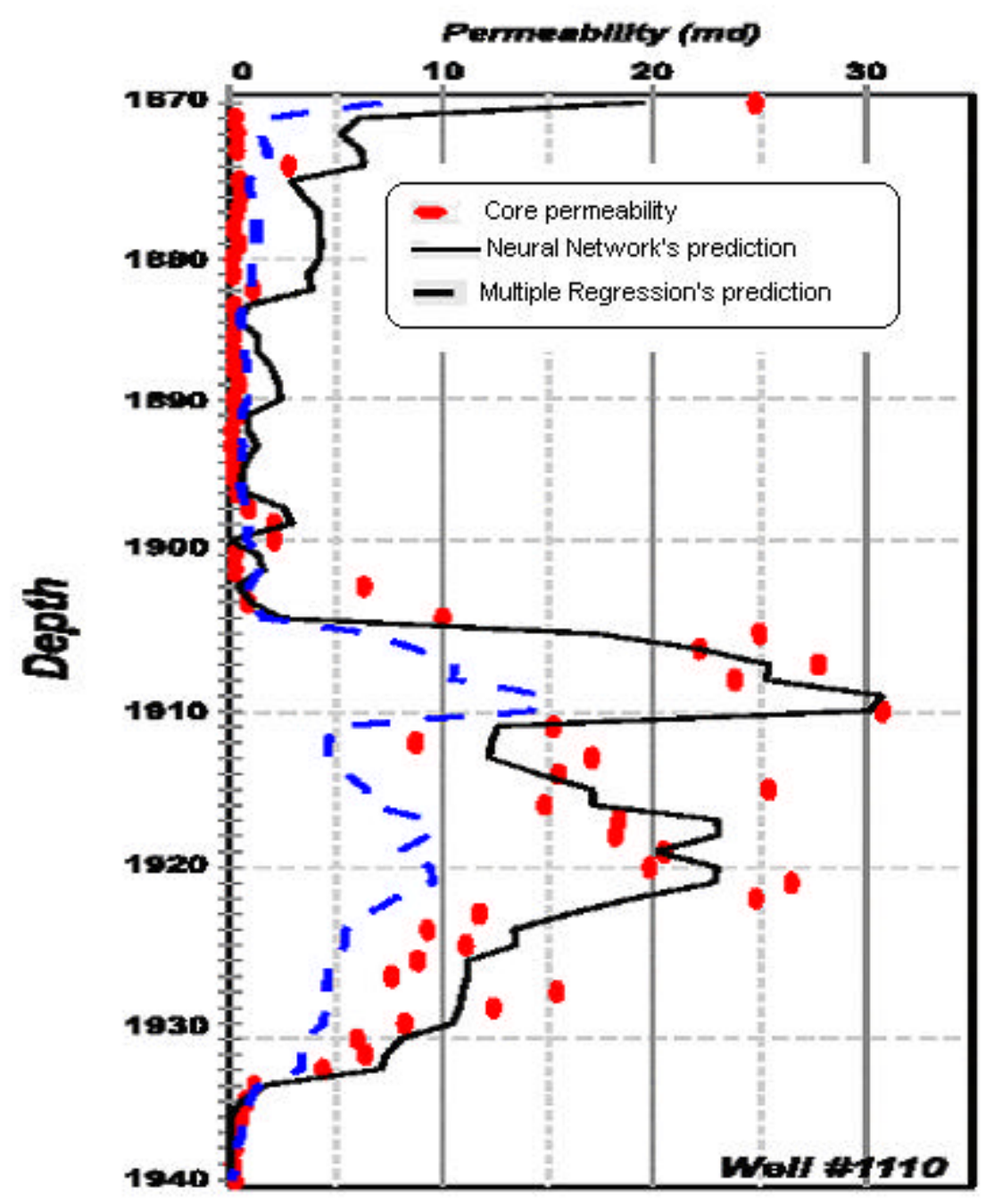

Figure 26 Prediction of the core permeability using Neural Networks methods, in comparison to the multiple regression methods [38]

Neural networks have been utilized to predict or virtually measure formation characteristics such as porosity, permeability and fluid saturation from conventional well logs [33]. Using well logs as input data coupled with core analysis of the corresponding depth, these reservoir characteristics were successfully predicted for a heterogeneous formation in West Virginia. It was shown that a carefully orchestrated neural network analysis is capable of providing more accurate and repeatable results when compared to methods used previously. 
Conventional wireline logs were used to generate synthetic Magnetic Resonance Imaging (MRI) logs to provide the log analyst with an additional tool in establishing reservoir characteristics [5]. Magnetic Resonance Imaging logs are well logs that use nuclear magnetic resonance to measure free fluid, irreductible water (MBVI), and effective porosity (MPHI) accurately. MRI logs can provide information that results in an increase in the recoverable reserve [33]. Because of the location of the wells, formation shape and formation lithology of the Cotton Valley, it was considered to be very effective if fuzzy systems are introduced as an intermediate tool to extract more knowledge from the available well logs. This information was used in developing artificial neural networks to predict MRI for different wells.

Virtual intelligence techniques (including Genetic Algorithms) were utilized to design optimum hydraulic fractures for the Clinton Sand in Northeast Ohio [34]. Twenty well were fractured/refractured each year but the lack of the detailed reservoir engineering data made impractical the use of 2D or 3D hydraulic fracture simulators. The wells with highest potential for post fracture deliverability enhancement were selected as the candidate wells. The combination of the design parameters identified for each well was also provided to the operator to be used as the guideline for achieving the well's potential. The most important parameters influencing storage well performance after refracture were also determined [32].

The fuzzy logic was used with success in the petroleum industry [35] and [5]. The fuzzy decision support system was able to capture one engineer knowledge with several years of experience. They predict the same well for restimulation and the restimulation process was a success.

One link between the surface seismic and sonic velocities was established by M.C. de Roots et al [43]. They were starting from the fact that the sonic velocity is a function of porosity, saturation and temperature. Neglecting the temperature effect (it can be assumed constant into a field) they tried to find the optimum relationship between pressure and saturation on one hand and the seismic velocity on the other. 
The methodology was as follows:

- the acoustic impedance of the overlying non-producing shales was obtained from well logs. The interwell values were determined using kriging

- from seismic the reflection coefficient was available and, combined to the previous point, they were able to determine the acoustic impedance of the pay zone

- from the acoustic impedance and the density (taken from reservoir simulation matching) P-wave velocity could be calculated

- they trained a neural network having for all the grid points:

$\circ$ the pressure from reservoir simulation (input)

$\circ$ the saturation from reservoir simulation (input)

○ the sonic velocity at that point from the method described above (output)

- once trained the neural network they were able to make a plot velocity vs. saturation and pressure.

The relationship is depicted in Figure 27.

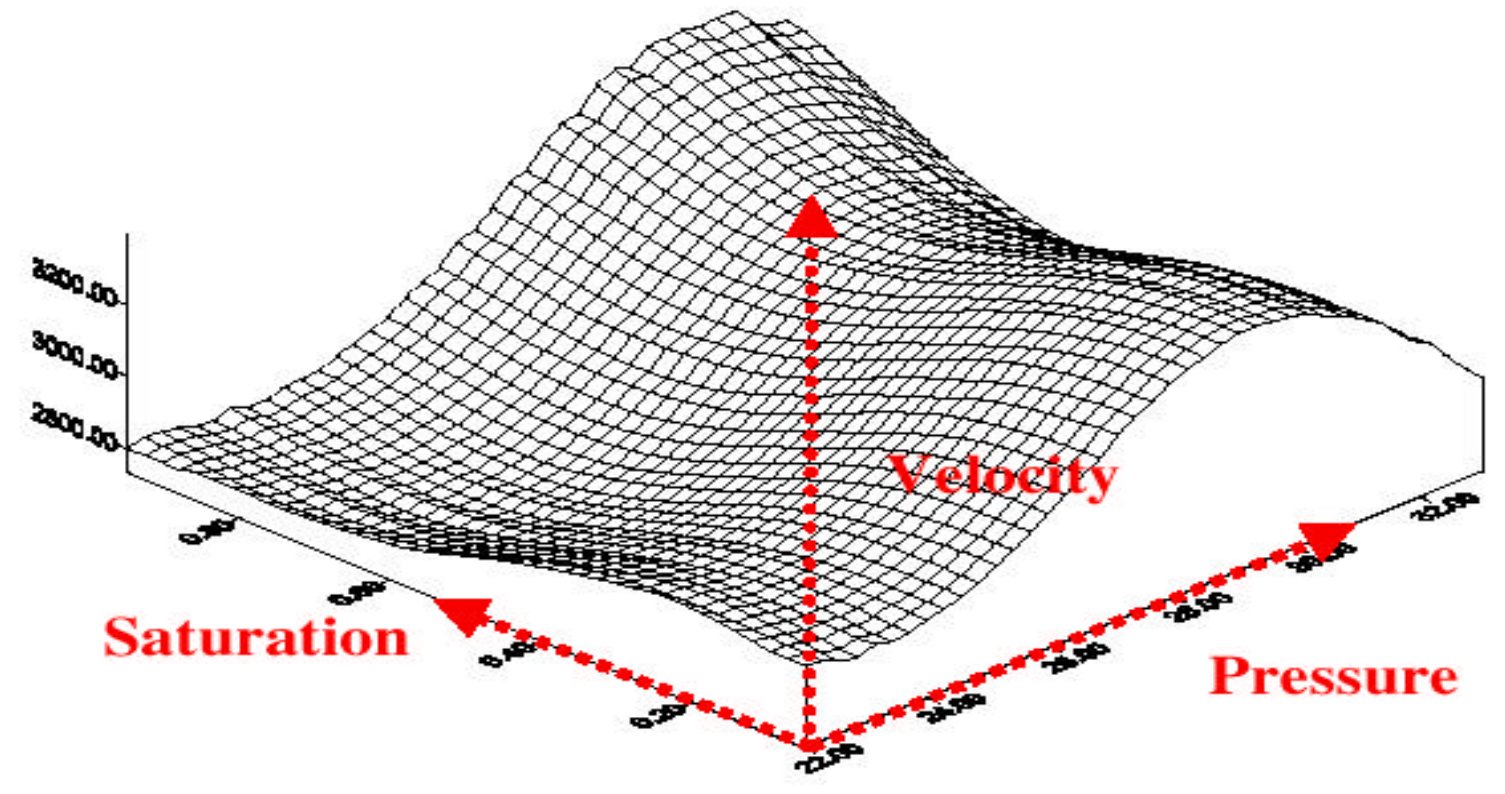

Figure 27 Seismic velocity $[\mathrm{m} / \mathrm{s}]$ as estimated by the neural network as a function of pressure [Mpa] and saturation 


\section{CHAPTER III - DIFFERENT WAYS TO ANALYZE THE SEISMIC EXPERIMENTS}

\section{III.1 LITERATURE REVIEW}

\section{I I.1.1 Different ways of analyzing the seismic experiments}

Paulsson et al [42] analyzed the same field as that taken in consideration in this project. They used the traveltimes as an input in a tomography inversion program. They noticed some differences between the tomography velocities and the velocities from logs; they assumed that this difference comes from the mud cake (quite possibly, the mudcake velocity is higher than that of the formation).

Wei et al [61] used the same field- Steepbank- but they used the information that the P and S velocities are proportional to one another. They used Jackson and Tweeton's (1994) MIGRATOM SIRT solver but rewrote the computer program code in $\mathrm{C}++$ (personal communication). They did the $\mathrm{P}$ and $\mathrm{S}$ tomographic reconstructions in parallel, with a constraint range set on acceptable values of Poisson's ratio at each step of the iteration to a final structure. When the velocity ratio was outside of the acceptable range, they applied a correction. Their model is slightly different comparing with Paulsson's model.

Lines et al [27] used the traveltime inversion to produce velocity tomograms. They used a relationship between the porosity and the sonic transit time (Figure 28) to predict the porosity from the velocity tomogram. 


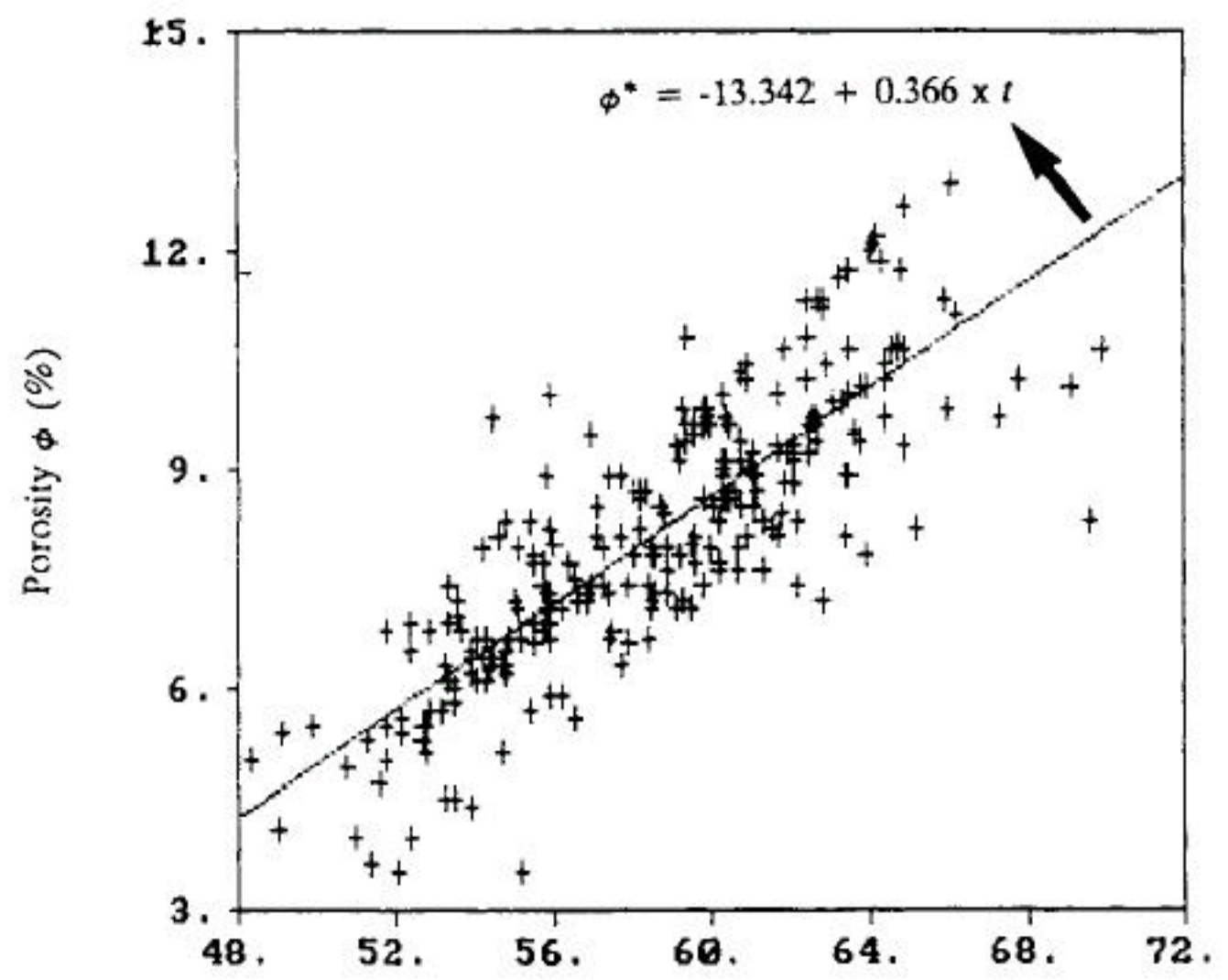

Sonic Transit Time $t$ ( $\mu \mathrm{s} /$ foot)

Figure 28 Plot of porosity versus slowness for 256 wells used in deriving velocity- porosity relationship [27]

Williamson et al [63] noticed the importance of the initial model (starting model) upon the final result. They figured out how important is this starting model and the constrains- the logs in wells.

To overcome the lack of detailed velocity resolution, Zhow et al [59] used a hybrid strategy of wave equation traveltime + waveform inversion. Instead of picking the traveltimes, they used the entire seismic traces- 2000 samples for each trace. They claim that, using this approach the resolution increased. 
Lee et al [25] proved that the crosswell data can be acquired with a $1500 \mathrm{ft}$ interwell spacing and over a depth range from 7700 to $9600 \mathrm{ft}$. Good quality and high frequency (>1000 Hz) data were acquired over most of the survey interval which contains massive limestones. However they could not acquire any useful data within the shale layers. They had identified three important factors that influence the data quality:

1. the noise level at the receiver position

2. the attenuation of the medium

3. the transmission loss and alteration in radiation pattern that occur at interfaces with a large impedance contrast.

Trappe and Hellmich proven in their work that a relationship between the seismic attributes and the rock properties can be found [55]. They found the porosity-thickness across the field using the following attributes:

1. Amplitude

2. Acoustic impedance

3. Lateral homogeneities at reservoir level

They validated their results removing one well at a time and trying to predict the value. The maximum difference was found when leaving the extreme low and high values out of the training set.

The 3-D seismic data were used as an input to a neural network to construct one depth maps for the top of the target L horizon of the Nash Draw field in southeastern New Mexico [19]. At Nash Draw the wells are confined to the central region of the seismic survey, and conventional geostatistics reliably interpolated depths only in the region defined by well control. The methodology used is: 
The MLP approach used the best three of 28 statistically ranked seismic attributes to predict the average velocity field from the surface to the L horizon.

$\checkmark$ Each map was constructed using 15 wells as control points with three wells excluded for testing. In that work, 46 seismic attributes were calculated using Landmark's Poststack Attribute Library (PAL) for a depth interval of ground-200 ms to the top of the L horizon.

$\checkmark$ Twenty-eight attributes were kept and another 18, which had edge effect problems were not further analyzed.

$\checkmark$ Being both statistically dangerous and not computationally feasible to use all 28 attributes to form a regression relationship for velocity, therefore a statistical technique based on a fuzzy-ranking algorithm was used to select attributes best suited for predicting velocity. The algorithm statistically determines how well a particular input (seismic attribute) resolves a particular output (reservoir property at the well bore) with respect to any number of other inputs. All 28 attributes were numerically ranked using the algorithm. The three best attributes were the average peak response frequency, the average absolute instantaneous frequency and the average instantaneous frequency.

They compared the depth maps created using the MLP with two other maps created using two software: TDQ and Z-map. The MLP was by far the best at predicting the three excluded testing points.

Crosswell seismic data from McElroy field, a Permian dolomite reservoir in West Texas, demonstrate that high-velocity and reflection images are obtainable in this carbonate reservoir [58]. They obtained a high-resolution tomographic image. They used this tomography and the cores and found that crosswell data, when integrated with porosity models based on log facies, add value to reservoir characterization. They noticed the difference in resolution between the surface seismic images and the crosswell seismic interpretation, the $2^{\text {nd }}$ giving more details (see their Figure 5 for more details). Cluster analysis was used to compare log and crosswell data and try to visualize relationships that were not apparent using other approaches. 


\section{III.1.2 The nonuniqueness of the traveltime tomography}

When compared with each other, the information regarding the accuracy of different software for crosswell seismic inversion shows that the subject is still under research.

Lines et all [28] shown that two different models built by Amoco and Schlumberger for the Grayburg unit, West Texas are pretty similar. Traveltime tomography results were obtained independently by the Amoco and Schlumberger groups. About 27,500 valid direct-arrival traveltimes were recorded in the tomography experiments. The cell sizes varied: the Amoco tomograms were $10 \mathrm{ft}$ vertical by $20 \mathrm{ft}$ horizontal, while the Schlumberger tomograms were $7 \mathrm{ft}$ vertical by $52 \mathrm{ft}$ horizontal. The tomography calculations were also based on different modeling and inversion methods. However, it was encouraging to note the similarities between the velocity tomograms. The main features of the tomograms show similar velocity variations as well as artifacts caused by aperture limitations.

In [26] the seismic tomography was acquired across a five-spot well pattern in order to visualize and understand important parameters controlling reservoir quality. Four separate tomography data sets were acquired inside the Self Unit of the Glennpool field. The acquisition and initial processing of this data were performed by Amoco Production Company under the direction of Larry Lines and Henry Tan of the Tulsa Research Center. The survey planning and interpretation was integrated with petroleum engineering and geological data.

For this project there were four tomography results available from The University of Tulsa, Amoco, Memorial University of Newfoundland and BOMTOM. The tomography software characteristics used in their study are presented in Table 1. The situation presented here is valid for 1994. 
Group Rays Anisotropy $\underline{\text { Access }}$

UTulsa curved Yes In Development

AMOCO Curved Yes Proprietary

MUN Curved Approximate Consortium

BOMTOM Straight No Public

Table 1 Properties of tomography software used in the Glennpool field

The Memorial University tomogram was significantly different from the Amoco result, particularly the level of detail in lateral variations of velocity- Figure 29. The University of Tulsa and BOMTOM tomograms are also different, as shown in Figure 30.

For this project, based on discussions with the geological and engineering teams, it was decided that the MUN result best represented the subsurface as tested by well control. 

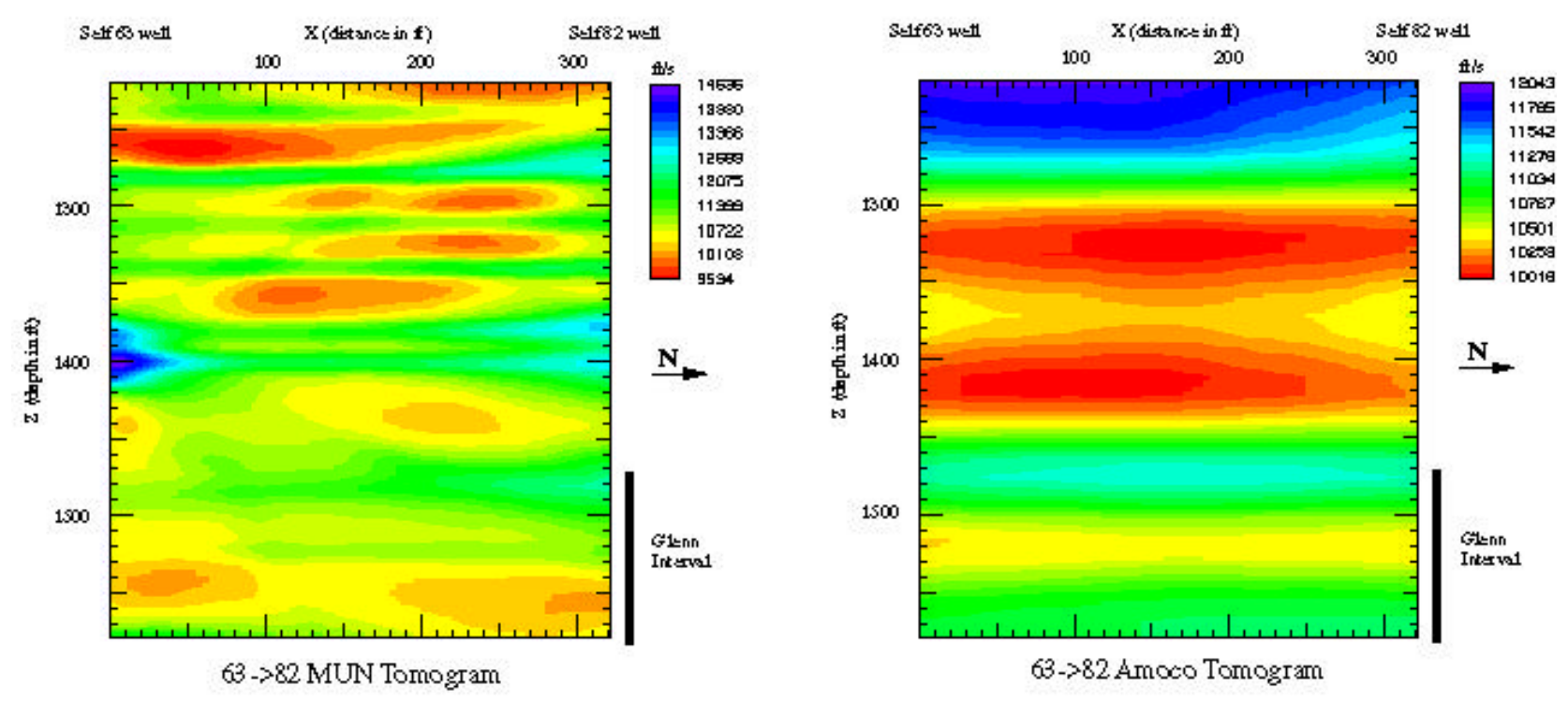

Figure 29 Comparison of MUN and Amoco tomograms [26]
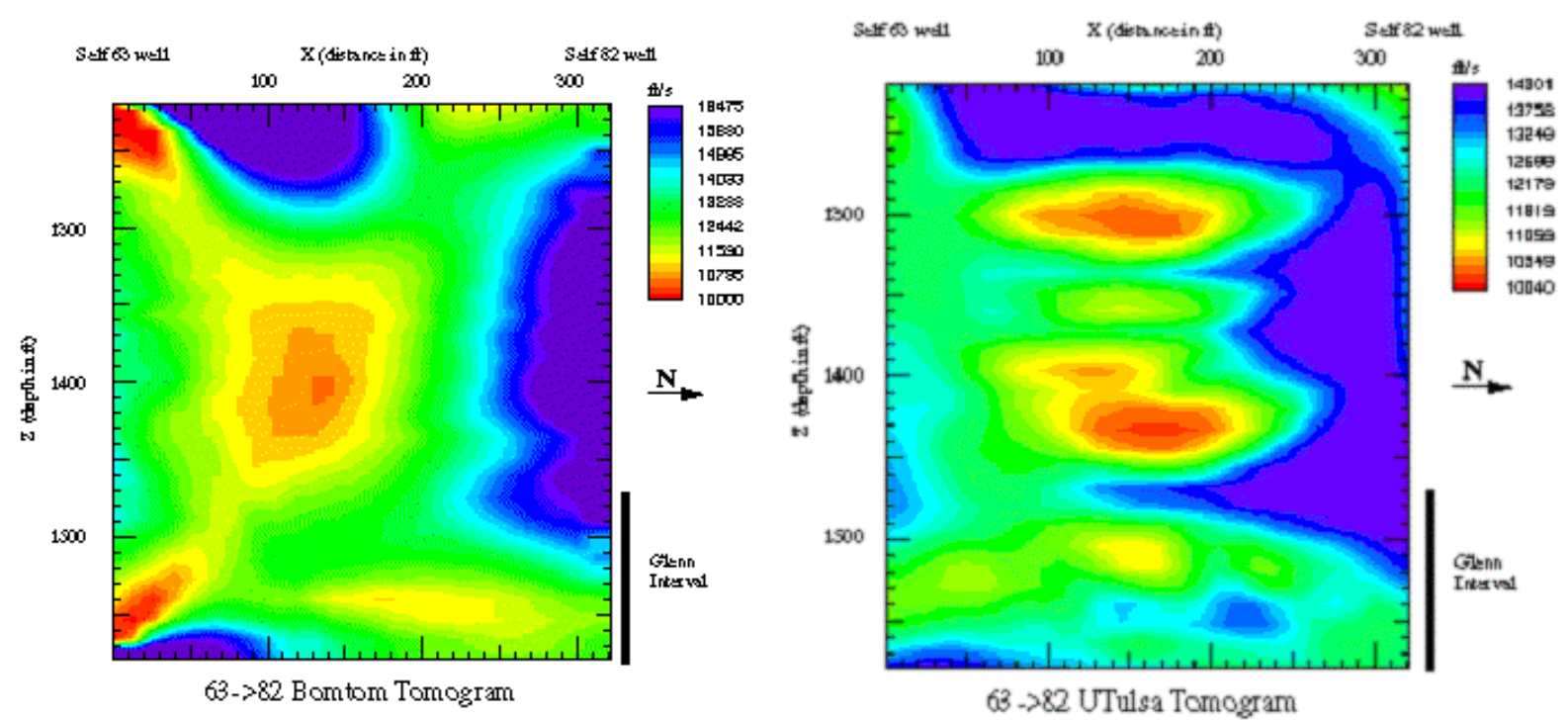

Figure 30 Comparison of BOMTOM and U. Tulsa tomograms [26]

The articles found in the literature regarding the crosswell seismic experiment at Steepbank (the field analyzed in this project)- [42] and [61] gives pretty similar results- Figure 31. Anyway, Liu and West [61] claim that $\mathrm{P}$ and $\mathrm{S}$ tomography helps to reduce ambiguity in either $\mathrm{P}$ and $\mathrm{S}$ data set alone- in their project the $\mathrm{P}$ and $\mathrm{S}$ wave velocities have been reconstructed together while constraining the acceptable range of Poisson's ratio. 

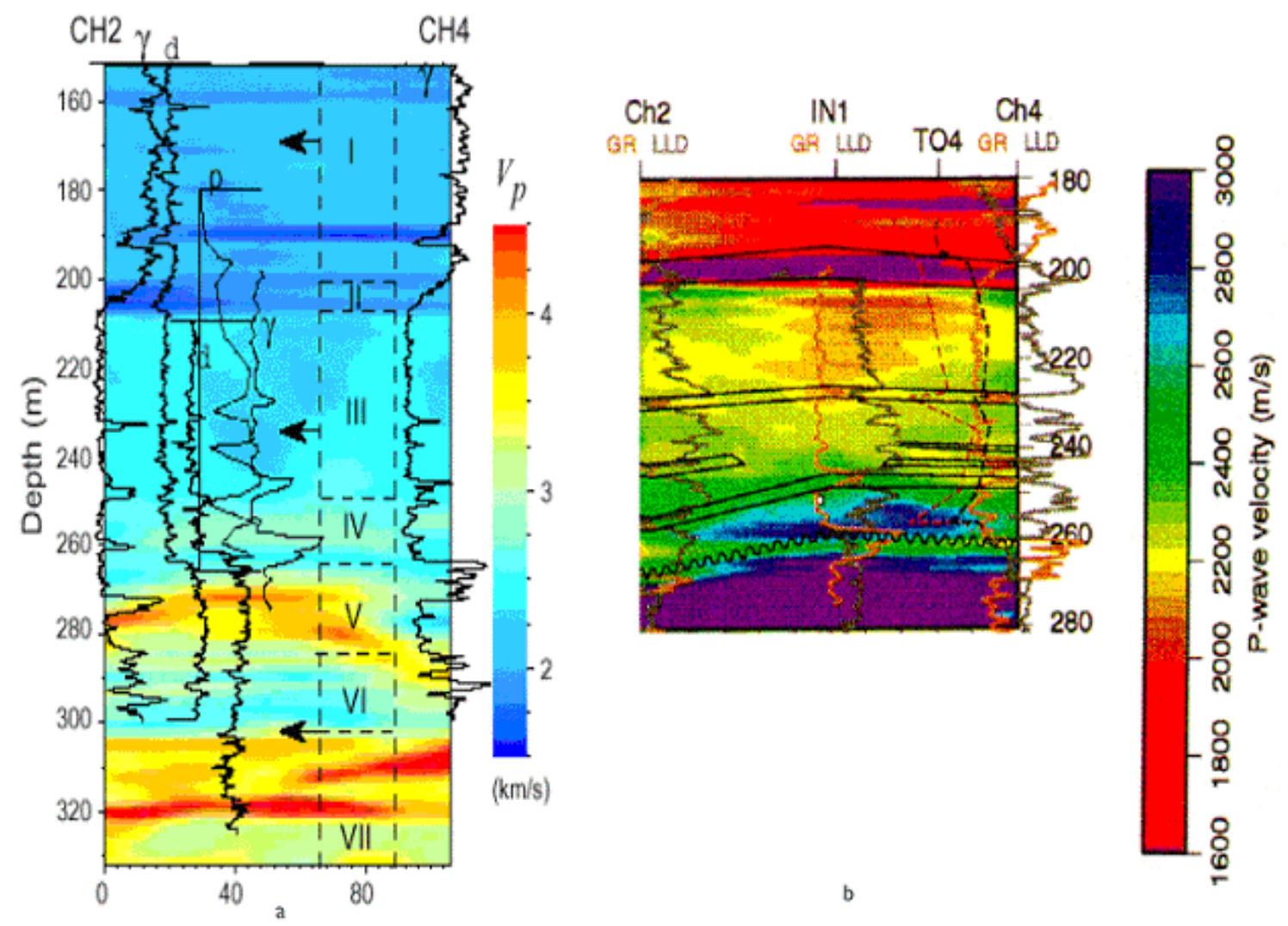

Figure 31 Comparison of two models regarding the line CH2- CH4 in the Steepbank crosswell seismic experiments- the [42] model (a) and the [61] model (b)

While all features of the seismic signals are directly caused by rock physics phenomena, the relationships between rock properties and the more obscure seismic attributes are not obvious. It is becoming increasingly difficult to derive attribute-property relationships directly from theory. In the presence of 3-D seismic data and logged wells, Schultz et al [47] found that the simultaneous analysis of seismic attributes with borehole data often leads to better estimates of reservoir or rock property distributions, compared to estimates generated only from well data (where the seismic data are used only for geometry or structure). 
In their examples, the attributes were averaged in a zone defined by two surfaces interpreted from logs as the top and bottom of the target layer. Because the layers were thin, the averaging zone of the seismic attribute was extended several tens of milliseconds below the lower surface. Equally, the log values must be averaged in some fashion over a vertical interval defined by formation boundaries.

For example they found a significant but highly non- linear relationship between the volume of clay and instantaneous frequency- Figure 32 . Inside the plot the names correspond to the wells analyzed. Since nonlinear relationships are unknown and varied, instead of prescribing a particular nonlinear model to perform the calibration (e.g., a polynomial typically used in regression), they let an artificial neural network learn a nonlinear model using example data.

When the calibration curve has been computed, it establishes a functional relationship to convert seismic attribute data to rock properties. They were able to plot the areal distribution of the volume of clay and shown that the level of detail is greater when the seismic guided estimates is involved in the analysis- Figure 33. To show that the methodology is better than simply kriging the known values, they used nine wells for the analysis and keep six wells as the verification (they were put aside).

They predicted effective porosity using the average acoustic impedance and the water saturation using the depth. Comparing the real values in those six wells with the values determined using two methods:

1. with seismic guidance

2. without seismic guidance

they found that the errors are reduced in the case of the seismic attribute guidance- Figure 34 . The best result gave an average error reduction of a factor of about two for porosity and about two and one half for water saturation. 


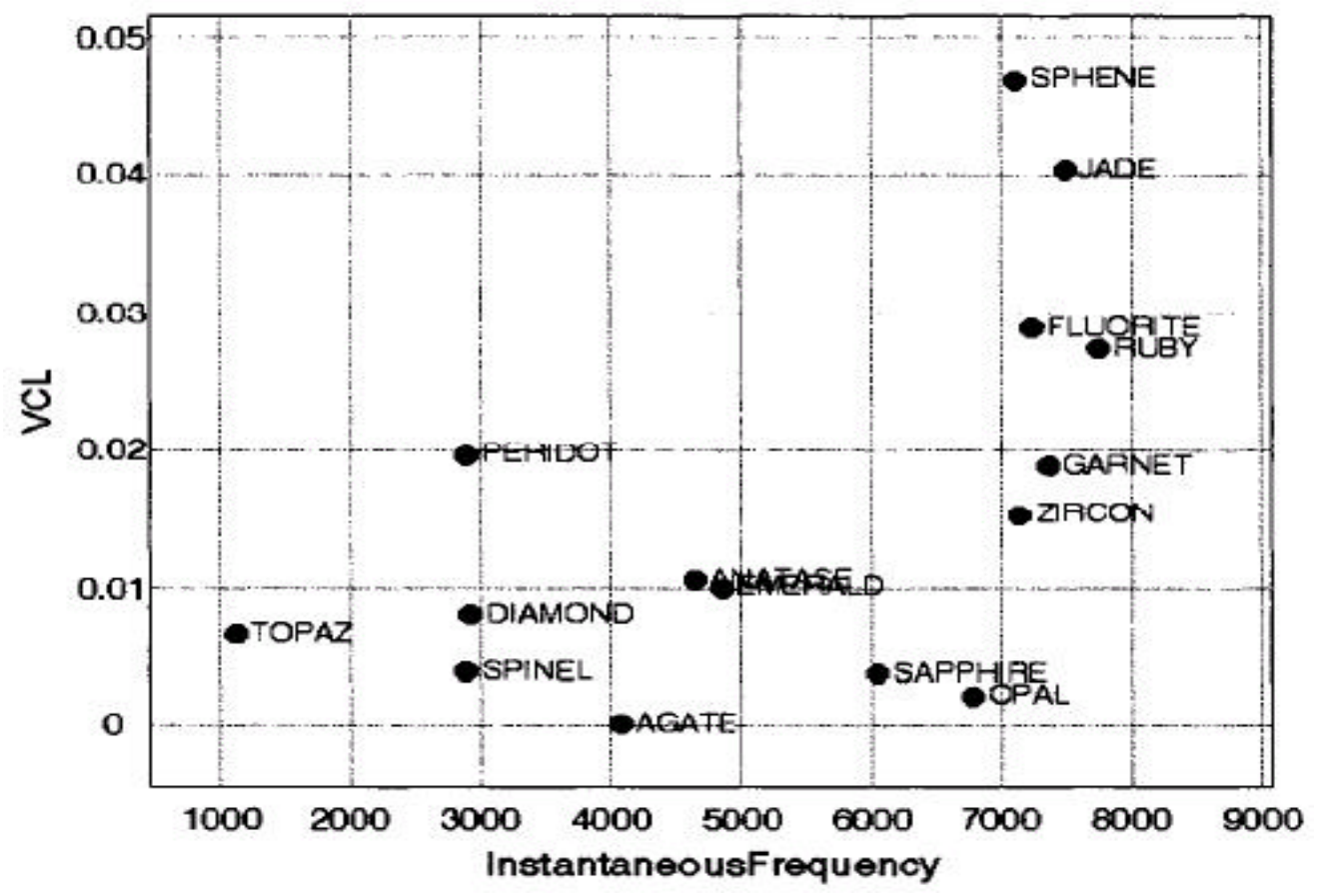

Figure 32 Instantaneous frequency attribute against the volume of clay log property [47]

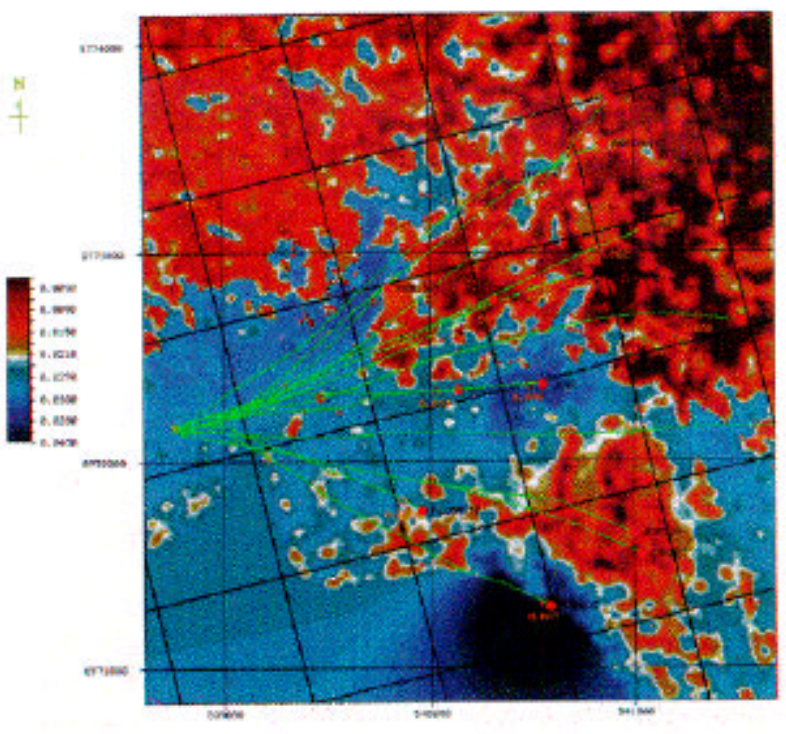

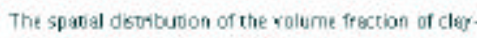

seismi: guided log property tstimwtion

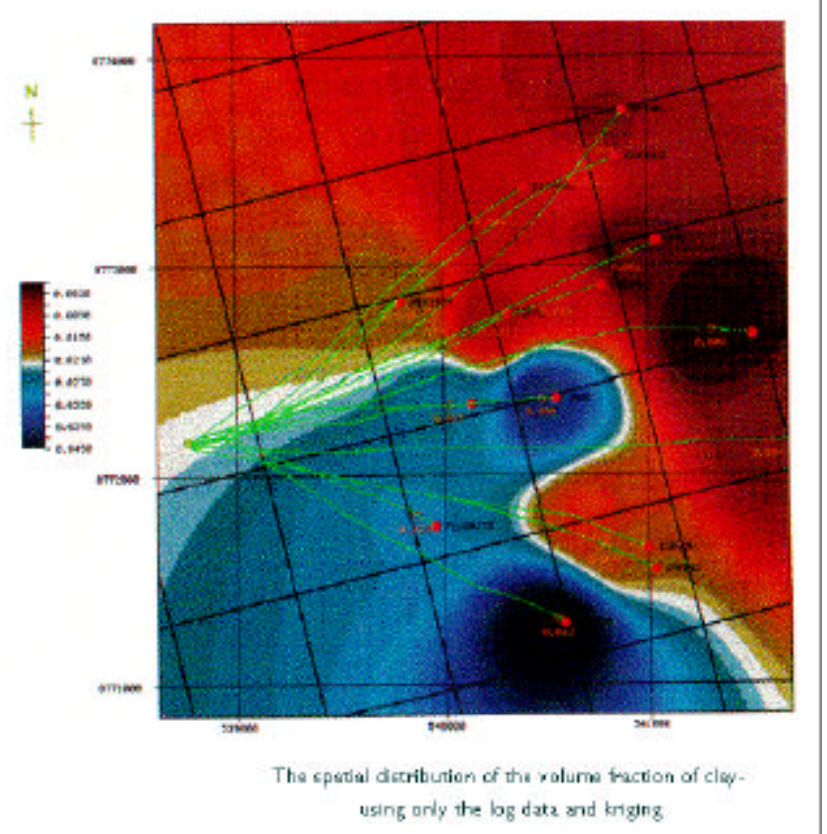

using only the log dizts and kriging

Figure 33 The spatial distribution of the volume fraction of the clay, with and without the seismic 


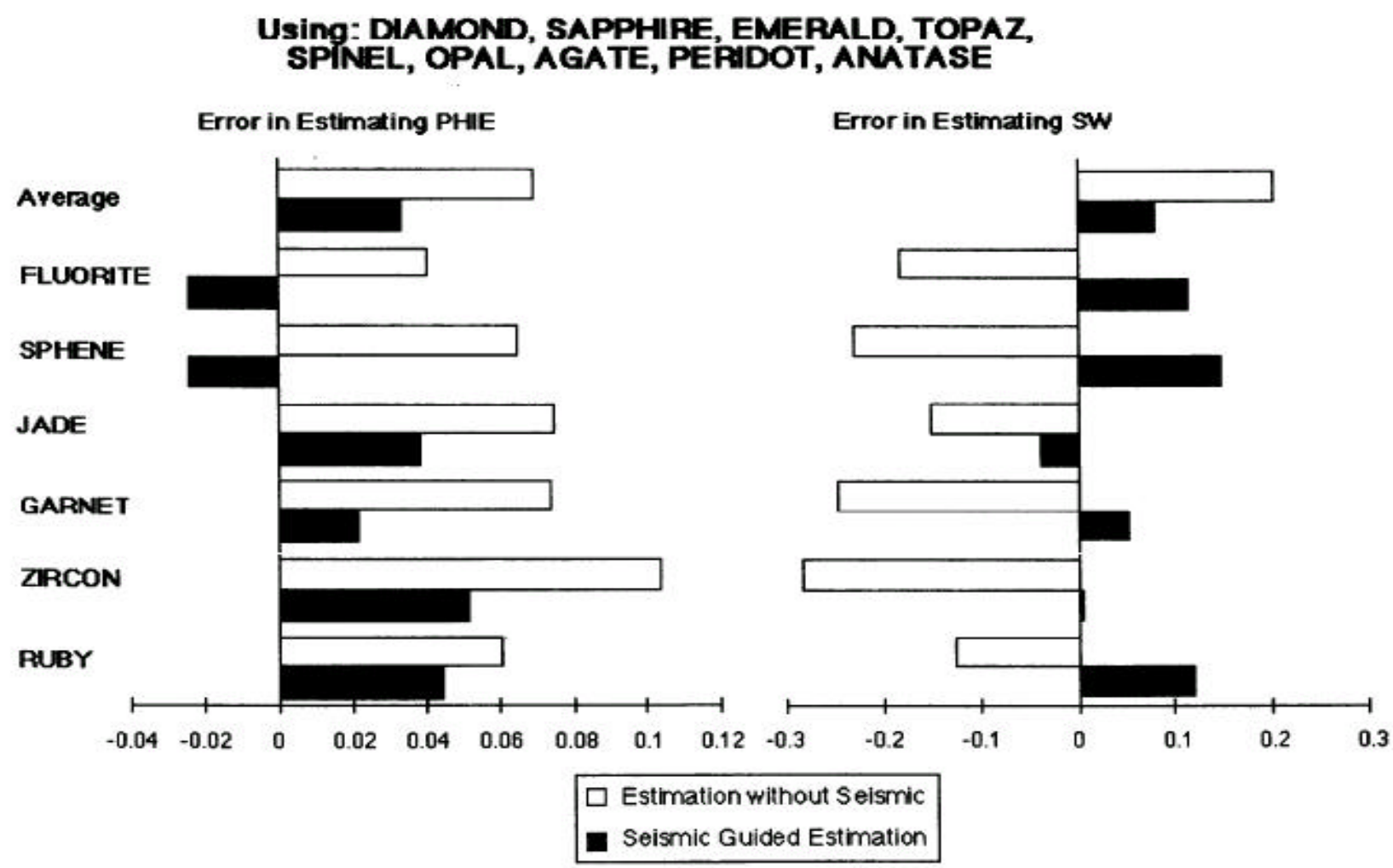

Figure 34 A comparison of estimation errors at each of the six validation wells for effective porosity (left) and water saturation (right) with and without seismic guidance

From their analysis it seems that further efforts to predict the zone properties inside the pay zone (as a function of depth) can lead to success and the work done in this project is a step forward.

The problem of forecasting the reservoir properties based on seismic attributes was analyzed in [3] too. For the Nash Draw field in SE New Mexico over 80 seismic attributes were extracted. They were averaged across the entire interval for the horizons of interest (the Brushy Canyon $\mathrm{K}$ and $\mathrm{L}$ sands). 
Instead of using all 80 attributes to form regression relationship, Balch developed a software based on a fuzzy- ranking algorithm to select attributes best suited for predicting individual reservoir properties. The algorithm statistically determines how well a particular input (seismic attribute) could resolve a particular output (reservoir property) at the wellbore. A neural network was later trained having as input the seismic attributes and as output a reservoir property (porosity, water saturation, net pay). They knew the values in 16 training wells and compared the real values with the predicted values in three wells kept for verification. The method shown good results in predicting the reservoir properties in the verification wells.

\section{I .1.3 The prediction of reservoir properties in depth}

There were found only a few references where the reservoir properties vs. depth were predicted but none of them discuss the problem of resolution. Also they did not use the advantages of the crosswell seismic experiments.

An idea similar to the worked performed by Lines in [27] were applied in [7]. The gamma ray response was predicted between the wells by training an Artificial Neural Network with actual gamma ray $\operatorname{logs}$ available at the wells and the interwell seismic data. Using the a priori information that the porosity is well correlated with the gamma ray response (Figure 35) Chavathe et al estimated the interwell porosity distribution. The training set was represented by the data in one well- the surface seismic attributes while the calibration set were represented by the data in the $2^{\text {nd }}$ well. Once the network trained they predicted the gamma ray for the entire plane between the source and the receiver. A verification set was not available. 


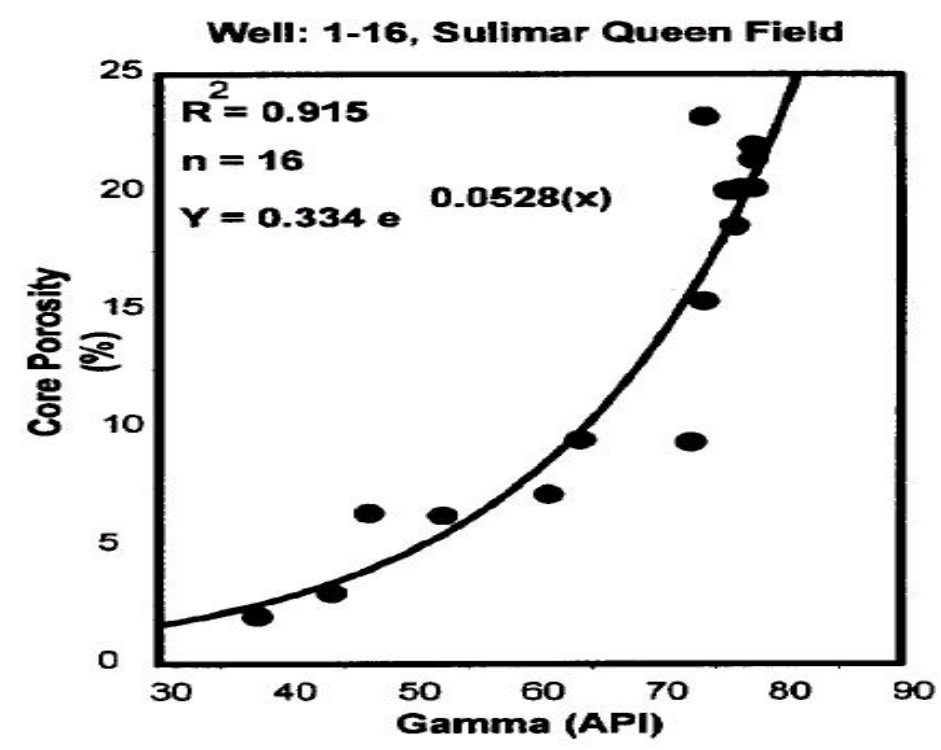

Figure 35 Core porosity and gamma ray correlation [7]

In the Soto and Holditch software (Oilfield Intelligence), the seismic attributes used as inputs in the neural networks were amplitude, phase, frequency, reflection strength and quadrature [50]. They tried to predict the gamma ray and the results seem to be acceptable for one well and good for the second well- Figure 36. They used the seismic attributes and the gamma ray $\log$ from 8 wells. Unfortunately this amount of information is not always available.
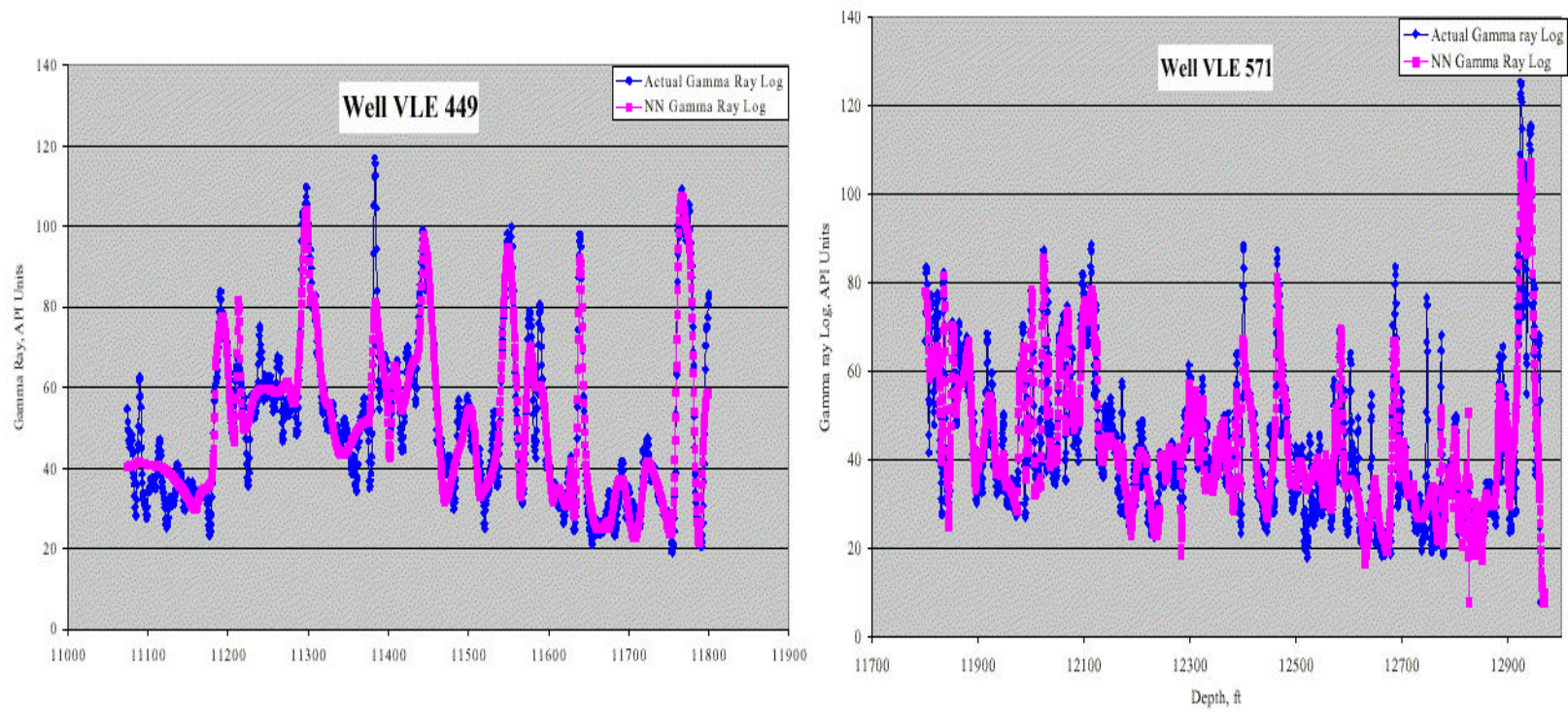

Figure 36 Comparison of actual and neural network Gamma Ray log for two wells predicted in [50] 
In [60] the input data consist of cores, well logs and fully processed 3-D poststack seismic available. The goal was to use a combination of rock physics modeling, seismic attribute generation and pattern recognition via neural network analysis- Figure 37 to determine the lithology into the field. They used the following reservoir characterization:

1. Pure shale; 2. Silty shale; 3. Interbedded sandstone-shale; 4. Massive wet sand; 5. Unconsolidated wet sand; 6. Planar laminated oil sand; 7. Unconsolidated oil sand; 8.Undefined.

In addition to the lithologic column output, multiple well log curves were provided as inputs. These curves were density, total porosity, Vp, Vs, clay volume, and water saturation. They calculated 16 different seismic attributes and used them along with well logs and lithology definition as input in the neural networks. The neural network gave lithology from logs with about $90 \%$ accuracy and was able to "recognize" the producing area.

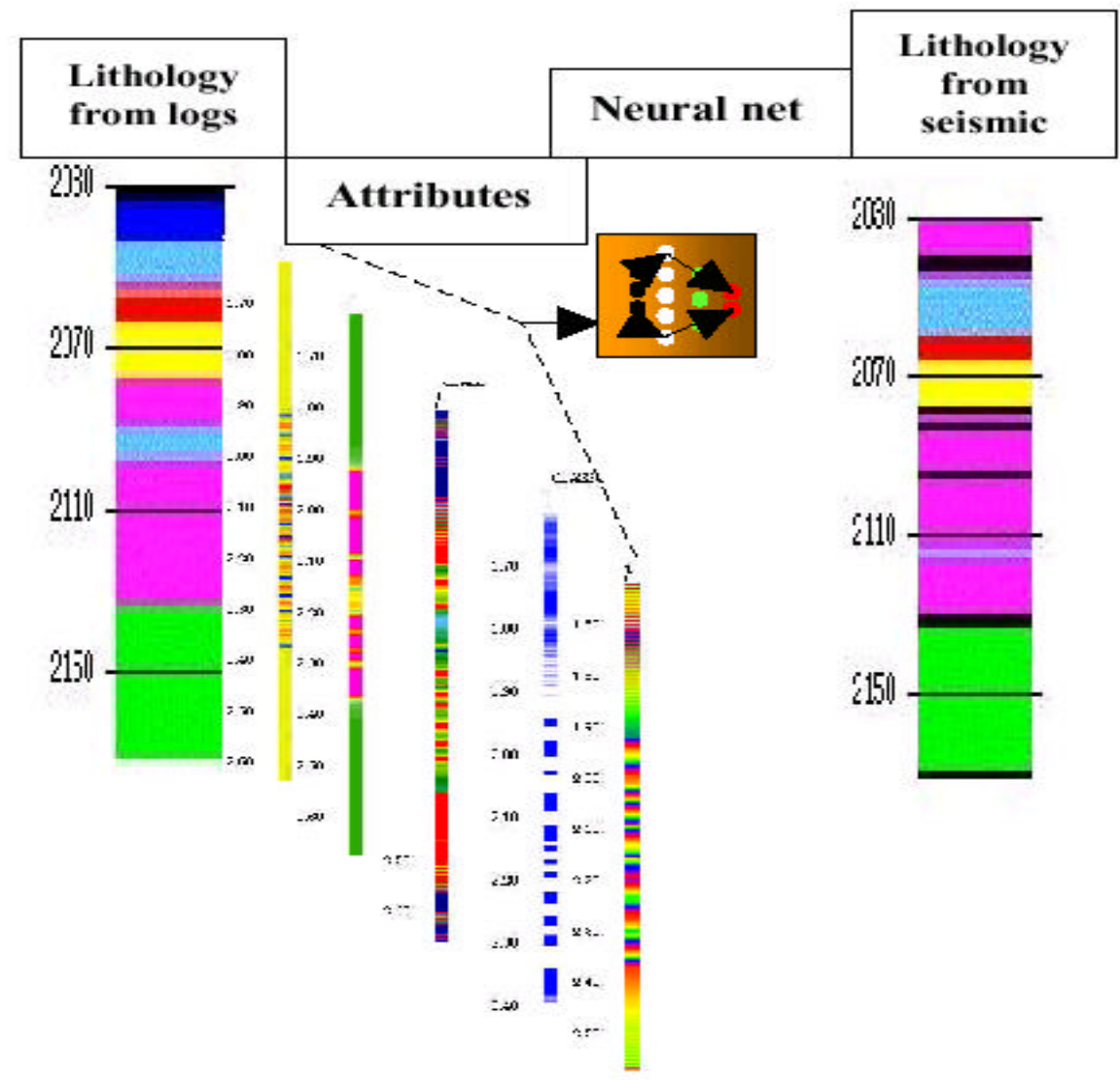

Figure 37 The neural network approach to predict the lithology in [50] 


\section{III.2 DISCUSSION}

As shown in the previous case histories the surface seismic was linked to the reservoir properties. By now the relationship was determined, in the majority of cases, for values averaged across the pay zone. In the few cases where the properties were determined, the property of the crosswell seismic to have a resolution between the surface seismic resolution and the log resolution were not used.

The next step is represented by the trial to determine the reservoir properties inside the pay zone with an increased resolution, the shape of such properties across the pay zone. The work done in this project is a starting point in this process. While the resolution of the surface seismic is low and decreases with depth it is obvious that the use of a method with a better resolution is necessary. So far, no work regarding this issue was found in the literature. Nobody tried by now to link the surface seismic experiments to the surface seismic and the literature doesn't contain any reference regarding this subject.

It is obvious that there can not be an analytical equation to relate the surface seismic to the crosswell seismic but the use of the Artificial Neural Networks can solve the problem.

A seismic trace with a better resolution can be created by using the crosswell seismic information. Later, further research that possible takes into consideration the methodologies presented in this paragraph could guess the reservoir properties variation in the volume of interest. We believe that the methodology presented in this work will increase the accuracy of the predictions. 


\section{III.3 WHY A DI FFERENT METHODOLOGY FOR TRAVELTI ME TOMOGRAPHY IS REQUI RED?}

In this project, a different approach to characterize the field and to predict the crosswell seismic experiments was used, the neural- networks models. The main reasons of this approach are the disadvantages of the other models:

1. The traveltime tomography seems to be susceptible to non-unique results

2. The raypaths are very complex

3. Even if the solution would be unique, the interpreted zone is still very small when it's compared with the field volume

4. The crosswell seismic experiments are very expensive; on the other hand, nobody consider the solution applied at Steepbank Canada to be applied in too many wells- they cemented the receivers in the space between tubing and casing.

5. The crosswell seismic experiment can be conducted in a small area. Once the network is trained, the crosswell seismic can be applied to the entire field

6. The crosswell seismic traces can be used in subsequent analysis, such as an input for another Artificial Neural Network

7. The crosswell seismic traces, once generated over the entire field, can increase the chance for a proper reservoir characterization

The disadvantage of the Neural Network approach is the fact that, if a facies was not encountered in the zone used for training, the Neural Network prediction is susceptible to large errors.

The idea of the next generation of "intelligent" software is depicted in the Figure 38. 


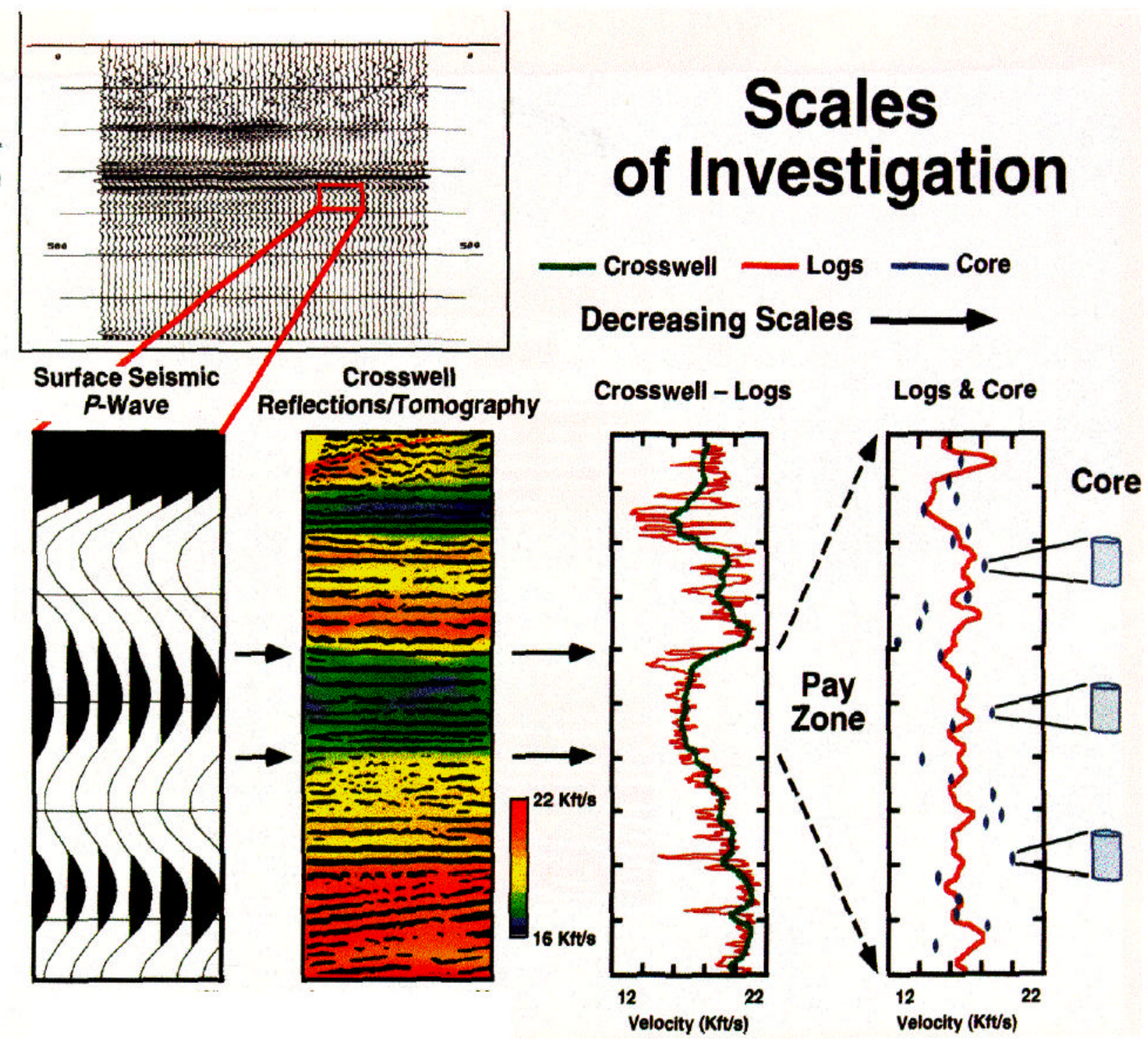

Figure 38 The main goal of the research in the petroleum area: use the crosswell seismic to predict the rock properties using its better resolution compared with surface seismic and the surface seismic areal coverage advantage 


\section{CHAPTER IV - THE FIELD DATA}

\section{IV.1STEEPBANK GEOLOGY}

The geologic model was developed using log information from all the wells at the site. The formations are almost flat lying- Figure 39, although the top of the Paleozoic carbonate is karsted and irregular [42].

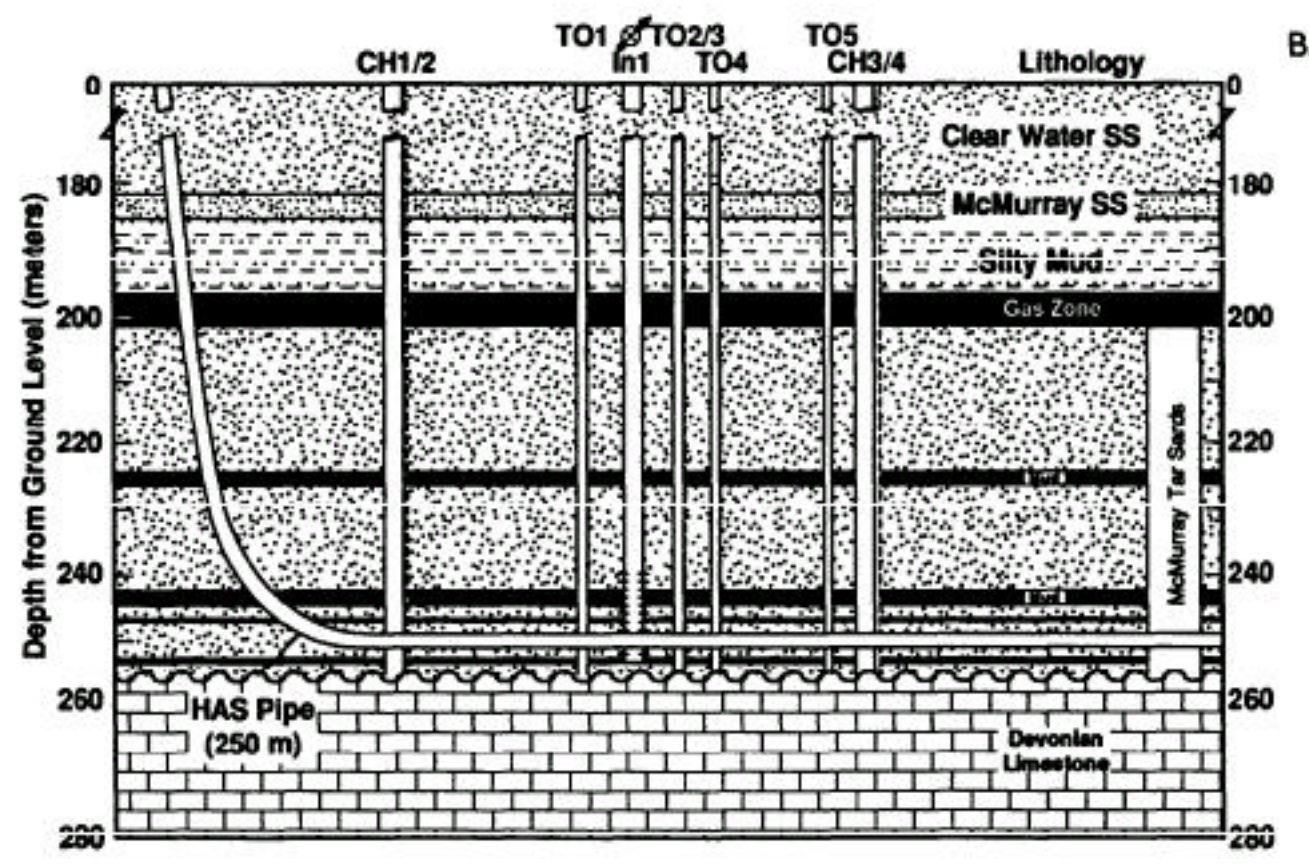

Figure 39 The field geology [42]

The Athabasca Tar Sands in northern Alberta contain large amounts of high viscosity petroleum in a shallow, nearly flat lying, Lower Cretaceous, sandstone stratum known as the McMurray formation. At Steepbank, about $60 \mathrm{~km} \mathrm{NE}$ of Fort McMurray, Alberta, Canada, the tar sand (P-wave velocity 2.1-2.3 km/s- [61]) is about $60 \mathrm{~m}$ thick and rests uncomfortably on Paleozoic Devonian limestone (Vp: 4.0-5.5 km/s) at a depth of about $264 \mathrm{~m}$ (w.r.t. a surface datum at $534.5 \mathrm{~m}$ above Mean Sea Level). It is overlain conformably by the sandy Wabiskaw member of the Lower Cretaceous Clearwater formation (Vp: $1.5 \mathrm{~km} / \mathrm{s}$ ) as well as more than $100 \mathrm{~m}$ of mixed 
sands and shales of the Grand Rapids formation (Vp: $2.0 \mathrm{~km} / \mathrm{s}$ ). The tar sand itself can be subdivided into several units beginning with an inhomogeneous, coarsely pyritic, basal unit of some $10 \mathrm{~m}$ which is overlain by about 15 to $20 \mathrm{~m}$ of "rich pay" in which the sand is heavily saturated with bitumen. This is overlain by about $25 \mathrm{~m}$ of sand in which the bitumen-saturation is gradational.

A low velocity gas cap ( $\mathrm{Vp}: 1.5 \mathrm{~km} / \mathrm{s}$ ) is at the top of the McMurray. The gas cap and the carbonate interface at the base of the McMurray produce strong velocity contrasts. Both greatly complicate the seismic data, since they cause strong refractions, reverberations, head waves, mode conversions and scattering. This, in turn, makes interpretation difficult, despite good data quality. The more important effect, however, is that the strong refractions lead to irregular ray paths so that portions of the field has sparse ray coverage.

The $V_{p}$ and $V_{s}$ velocities for the well CH4 and typical waves are presented below (Figure 40).

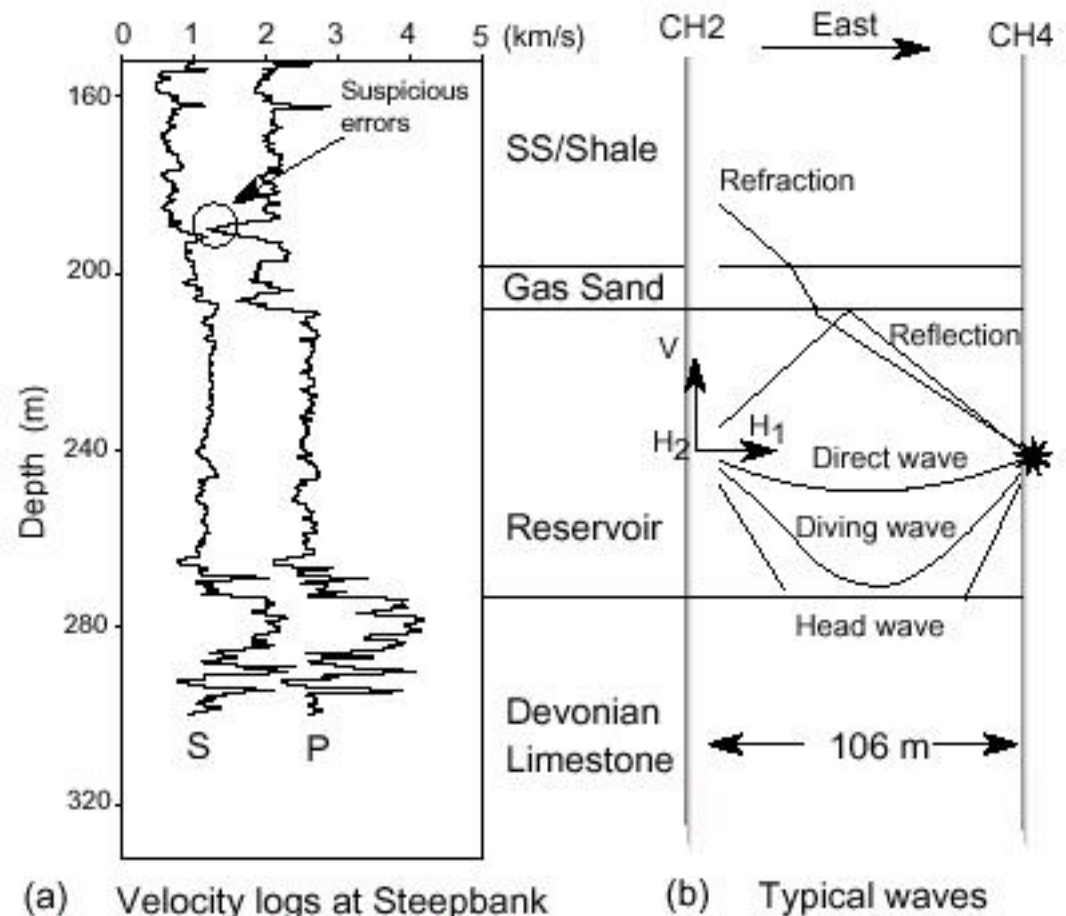

Figure 40 Sonic log velocities and typical wave path from one well to another 


\section{IV.2 HASDrive process}

The acronym stands for Heated Annulus Steam Drive.

At this depth, bitumen has to be extracted by some sort of in-situ recovery process. As a pilot project, it was installed a Steam-Assisted Oil Recovery (SAOR) system at Steepbank. A horizontal well (HASP) was drilled along at a depth of about $255 \mathrm{~m}$ near the base of the tar sand, and steam was circulated in it to preheat an annulus surrounding it. Steam was then injected directly into the base of the tar sand near the HASP from a muzzle on a vertical injection well (INl). A vertical collector well was located about $200 \mathrm{~m} \mathrm{SE}$ of the injector along the HASP. Tomography and other borehole monitoring were concentrated around the injector (Figure 41).

All wells were drilled as close to the horizontal well as possible to ensure that steam injection and oil production were within the preheated zone. Wells TO1- TO5 were drilled for temperature observations.

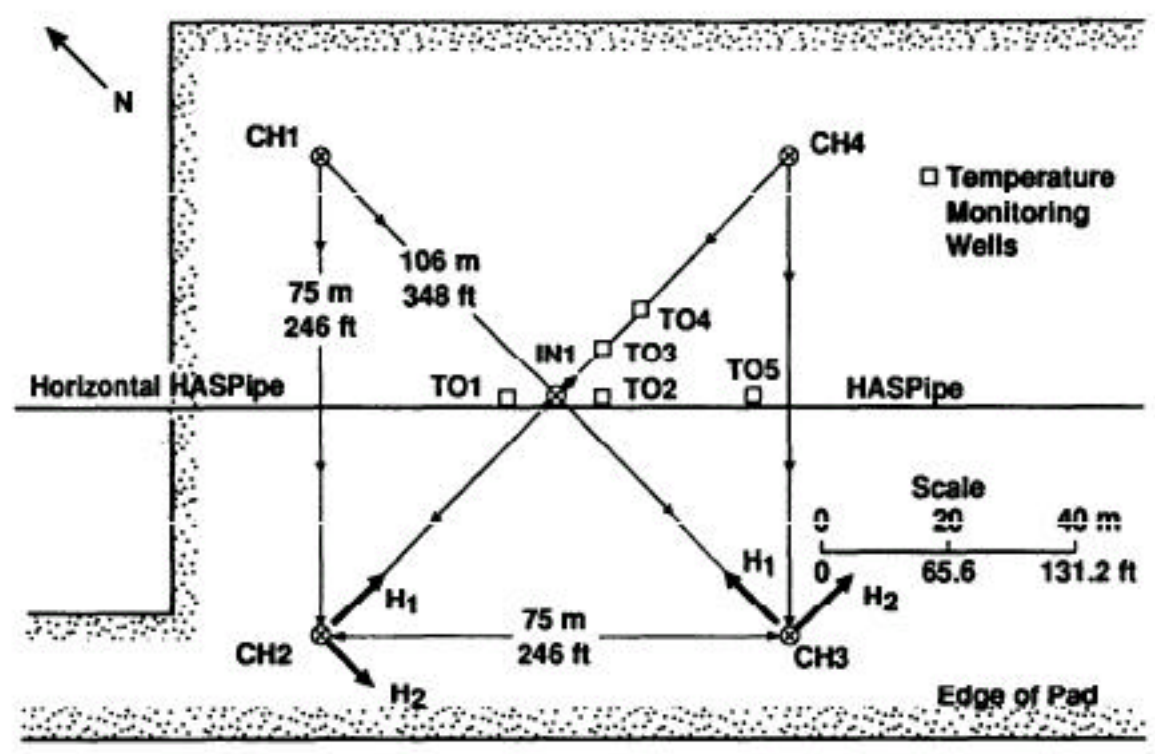

Figure 41 HASDrive process 


\section{IV.3 THE SURFACE SEI SMI C EXPERI MENT}

Figure 42 shows a map view of the survey. The small and midsize numbers represent the 231 geophone and 119 shot locations of the surface survey. Each shot was recorded by all the receivers, which remained fixed during the entire survey.

The source for the surface survey was $0.5 \mathrm{~kg}$. of dynamite planted in a single 10 -meter hole. The receiver stations consisted of a single geophone planted in a 1-3 meter hole. Both the shot and receiver intervals were 8 meters. These survey parameters were selected in order to record the broadest possible range of frequencies. The wavelengths in the surface data are as short as 16 meters, so the effort was a successful one.

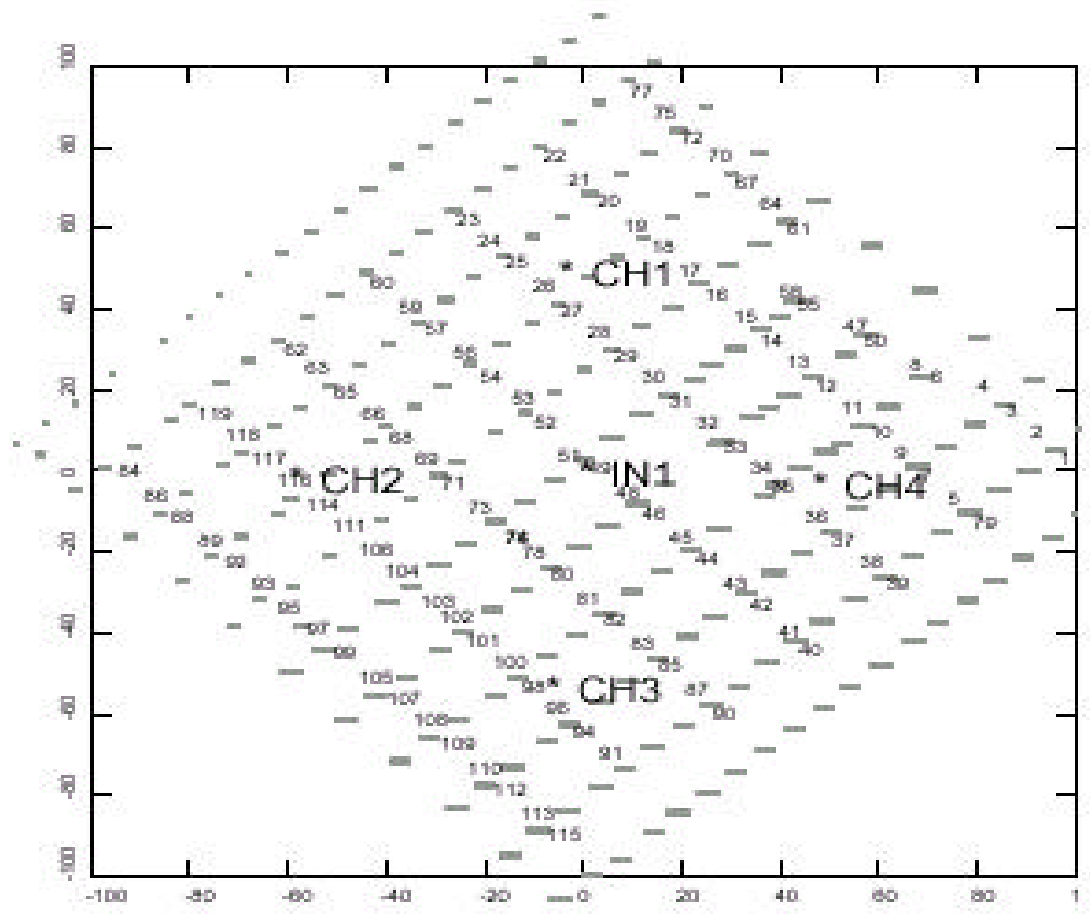

Figure 42 Map of 3-d surface survey and wells used in crosswell survey. Small numbers are receiver locations; medium size numbers are shot locations 


\section{V.4 FI ELD PROCEDURES}

Time lapse tomography was carried by permanently cementing 80 element linear arrays of three component geophones into two vertical wells $(\mathrm{CH} 2$ and $\mathrm{CH} 3)$ and then operating a source at various heights in two other vertical holes (CH1 and $\mathrm{CH} 4)$.

The crosshole survey wells are also shown in Figure 42 as well as in Figure 48. They are located at the corners of a square, $75 \mathrm{~m}$ on a side and approximately centered on injection well IN1. CH1 and $\mathrm{CH} 4$ were the source wells, $\mathrm{CH} 2$ and $\mathrm{CH} 3$ the receiver wells. Recording each shot into both receiver wells resulted in four tomography sections, two intersecting IN1 along the diagonals of the square and two along its northwest and southeast edges. Source and receiver depth intervals were $2.0 \mathrm{~m}$. There were a total of 80 source and receiver levels, ranging from 160 to $318 \mathrm{~m}$ below ground.

The seismic source was the vertical, borehole-clamped, hydraulic vibrator developed at Chevron. It is analogous to surface vibroseis; the clamp acts as the baseplate and "drive" comes from a vertically vibrating reaction mass suspended below the clamp. The hydraulic pump and motor are at the surface; hydraulic fluid is fed to the actuator through hoses which, along with an electrical cable, are clamped to the wireline.

Two 80-level, three-component geophone arrays were cemented into $\mathrm{CH} 2$ and $\mathrm{CH} 3$. The arrays were clamped to the outside of the tubing and lowered down through the casing. Cement was pumped through the tubing and around the geophones in the annulus between tubing and casing. Displacing the cement inside the tubing allowed the receiver wells to remain available for temperature observations and other wireline surveys.

Geophones were carefully oriented so one horizontal component pointed along the diagonal of the square in the direction of the injection well. Depth of each geophone was verified by placing a radioactive cobalt marker on the three-component geophone pod which could, after deployment, be detected by a gamma ray survey. 
Deployment went smoothly. Pod depths were verified and particle motion plots of incident P-waves indicated that geophones were within five degrees of their intended orientation. The 80level arrays provided two important benefits. They increased the rate of data acquisition since, in principle, an entire shot fan could be recorded in one shot.

Almost as important was the improvement in data quality. The cement provided perfect coupling to the formation. Tube wavenoise, a universal problem for liquid-filled borehole measurements, was nonexistent; the response of the vertical and horizontal components, usually quite different with mechanically clamped geophones, was nearly identical.

Using these higher quality receiver arrays also resulted in significant cost savings. The cost of manufacturing and deploying the arrays was about $\$ 100$ 000. Typical recording costs for a crosswell survey are about $\$ 10000$ per day, so the arrays become cost effective after saving 10 days of survey time.

Two sweeps per depth position were sufficient for good signal-to-noise ratio. The sweep frequencies varied linearly from 10 to $640 \mathrm{~Hz}$. over a duration of 15 seconds. The depth range and vibrator point intervals were 160-318 and 2 meters, respectively.

Only the vertical component of the crosswell data was used in order to be consistent with the vertical component geophones used in the surface survey. 


\section{IV.5 DATA PROCESSI NG.}

Processing of the surface data consisted of prestack Kirchhoff depth migration followed by a 2040 - 250-500 band pass filter [13]. These numbers mean:

- full attenuation for frequencies below $20 \mathrm{~Hz}$. and above $500 \mathrm{~Hz}$.

- $3 \mathrm{db}$ attenuation at $40 \mathrm{~Hz}$. and $250 \mathrm{~Hz}$.

- no attenuation between frequencies of 40 and $250 \mathrm{~Hz}$.

Data adaptive filters such as deconvolution were not performed in order to keep the surface data consistent with the crosswell data.

Also, a visual inspection of the surface data did not reveal any statics problems. The ground was frozen at the time of the surveys, and the water table is right at the surface.

The crosswell data processing comprised separation of the up and downgoing reflections via an F-K filter. This was filtered by two separate Kirchhoff depth migrations, one for the upgoing reflections and one for the downgoing reflections. The polarity of the upgoing reflections was reversed, and the two migration products were combined.

Because of the small size of both the surface and crosswell surveys the migration had to be modified somewhat. Here data are migrated only to specular reflection points under the assumption of horizontal reflectors. This is similar to a VSP-CDP transform, although we are applying it to surface as well as crosswell data.

The velocity model used for migration was determined by linearly interpolating across the sonic logs from the 5 wells - the 4 used in the crosswell survey plus the injection well IN1 [13]. A constant velocity of $1700 \mathrm{~m} / \mathrm{sec}$ (based on the first breaks in the surface data) was chosen for the interval between the surface and 80 meters, the top of the sonic logs. 
Finally two lines were carved from the migrated surface data that coincide with the crosswell lines between $\mathrm{CH} 4-\mathrm{CH} 2$ and $\mathrm{CH} 1-\mathrm{CH} 3$.

The reflection point spacing for both the crosswell and surface data is 2 meters, and the vertical sample interval is 0.5 meters.

\section{V.6SYNTHETI C SEI SMI C TRACES}

Synthetic seismic traces were computed from the sonic log in IN1, which is located close to the intersection of the crosswell lines. The log top for IN1 is 204 meters, so the sonic log from $\mathrm{CH} 4$ was appended to the top of IN1 in order to extend the log coverage up to 80 meters. The traces were then filtered with a zero-phase, 20-40-150-300 Hz. band-pass filter so as to visually match the bandwidth of the seismic data. 


\section{V.7TRACE ATTRI BUTES}

\section{IV.7.1 Attributes computed}

Various trace attributes were computed from the seismograms. They include:

1. Acoustic impedance, a running sum of the reflection coefficient traces

2. Conventional amplitudes, a relative measure of reflection coefficients

3. Instantaneous phase: arctan. of Hilbert Transform of trace divided by trace

4. Instantaneous frequency: time-derivative of instantaneous phase

5. Trace envelope: magnitude of the trace and its Hilbert Transform

\section{IV.7.2 Trace Attributes analysis}

\section{IV.7.2.1 Conventional Amplitudes}

The processing done in [13] does not preserve true amplitude. Consequently the final migrated traces were scaled to have the same r.m.s amplitude as the synthetic trace, within a number of relatively small depth intervals. The amplitudes of the synthetic trace are proportional to the reflection coefficients as computed from the sonic log, so the seismic amplitudes are an approximation of the relative reflection coefficients. The numbers were scaled to have a maximum value of 10000 and written to file as integer numbers. 
The density log was not included in the computation of reflection coefficients since the resulting synthetic trace did not agree well with the real seismic data. The region of disagreement is within the reservoir between about 200 and 260 meters where strong events on the synthetic trace have no counterparts in the real data. The very unconsolidated nature of the reservoir and the possible dissolution of gas close to the wellbore may have biased the density log.

\section{IV.7.2.2 Acoustic I mpedance}

It can be shown that a running sum of the reflection coefficient trace is a good approximation to the $\log$ of the acoustic impedance, albeit limited to the frequency bandwidth of the trace itself. That is, the low-frequency trend of the acoustic impedance, such as would exist from the normal increase in velocity and density with depth, is missing. Quite often this low frequency trend is supplied by well logs or by velocities computed from the seismic data.

The running sum rationale is as follows:

The reflection coefficient at the i-th boundary is defined as:

$$
R C(i)=\frac{\left(\rho V_{p}\right)_{i+1}-\left(\rho V_{p}\right)_{i}^{i}}{\left(\rho V_{p}\right)_{i+1}+\left(\rho V_{p}\right)_{i}}
$$

where:

$\rho_{\mathrm{i}}=$ density of the i-th layer,

$\mathrm{Vp}_{\mathrm{i}} \quad$ = P-wave velocity of the $\mathrm{i}$-th layer

If the difference in velocity and density between the two layers is small, then the reflection coefficient can be written as follows:

$$
R C(i)=0.5 \frac{\Delta(\rho V p)}{\rho V p}
$$


Integrating this gives:

$$
\int R C=0.5 \ln (\rho V p)+C
$$

where $\rho V_{p}$ is the acoustic impedance, and $C$ is an arbitrary constant of integration. It would represent the acoustic impedance of the surface layer, but here we can set it to zero.

Replacing the integral with a summation, and assuming the trace is a measure of reflection coefficient strength, then the log of the acoustic impedance is proportional to a running sum of the trace.

Exponentiating the sum to get the actual acoustic impedance creates huge magnitude variations within the trace, so for visual appeal it is better to leave the result scaled to the logarithm. Because $\mathrm{C}$ was set to zero, the trace will have negative values.

Acoustic impedances are layer properties whereas reflection coefficients are interface properties, so it is sometimes helpful to look at the trace in both ways. The band limited nature of the acoustic impedances give them the same oscillatory character as the reflection coefficients, but the interpreter can take that into account and sometimes gain additional insight from this seemingly trivial operation.

\section{V.7.2.3 Instantaneous Phase}

To avoid a decimal point and to maintain a good dynamic range, the phase values were multiplied by 1000 and written to file as an integer number. 


\section{IV.8OUTPUT FORMAT}

The results of the data analysis was available in two forms [13]:

$>$ Graphical form- pdf files

$>$ Text file

\section{IV.8.1 The graphical form}

Synthetic seismic traces were computed from the sonic log in IN1, which is located close to the intersection of the crosswell lines. The log top for IN1 is 204 meters, so the sonic log from $\mathrm{CH} 4$ was appended to the top of IN1 in order to extend the log coverage up to 80 meters. The traces were then filtered with a zero-phase, 20-40-150-300 Hz. band-pass filter so as to visually match the bandwidth of the seismic data.

The first 10 traces on each plot are repeat copies of the synthetic trace computed from the log. Ten traces are shown, rather than a single trace, in order to provide the lateral coherence that helps to see the dominant reflectors. The shape of the synthetic seismic traces for the $\mathrm{CH} 2-\mathrm{CH} 4$ line is presented in the Figure 43. 

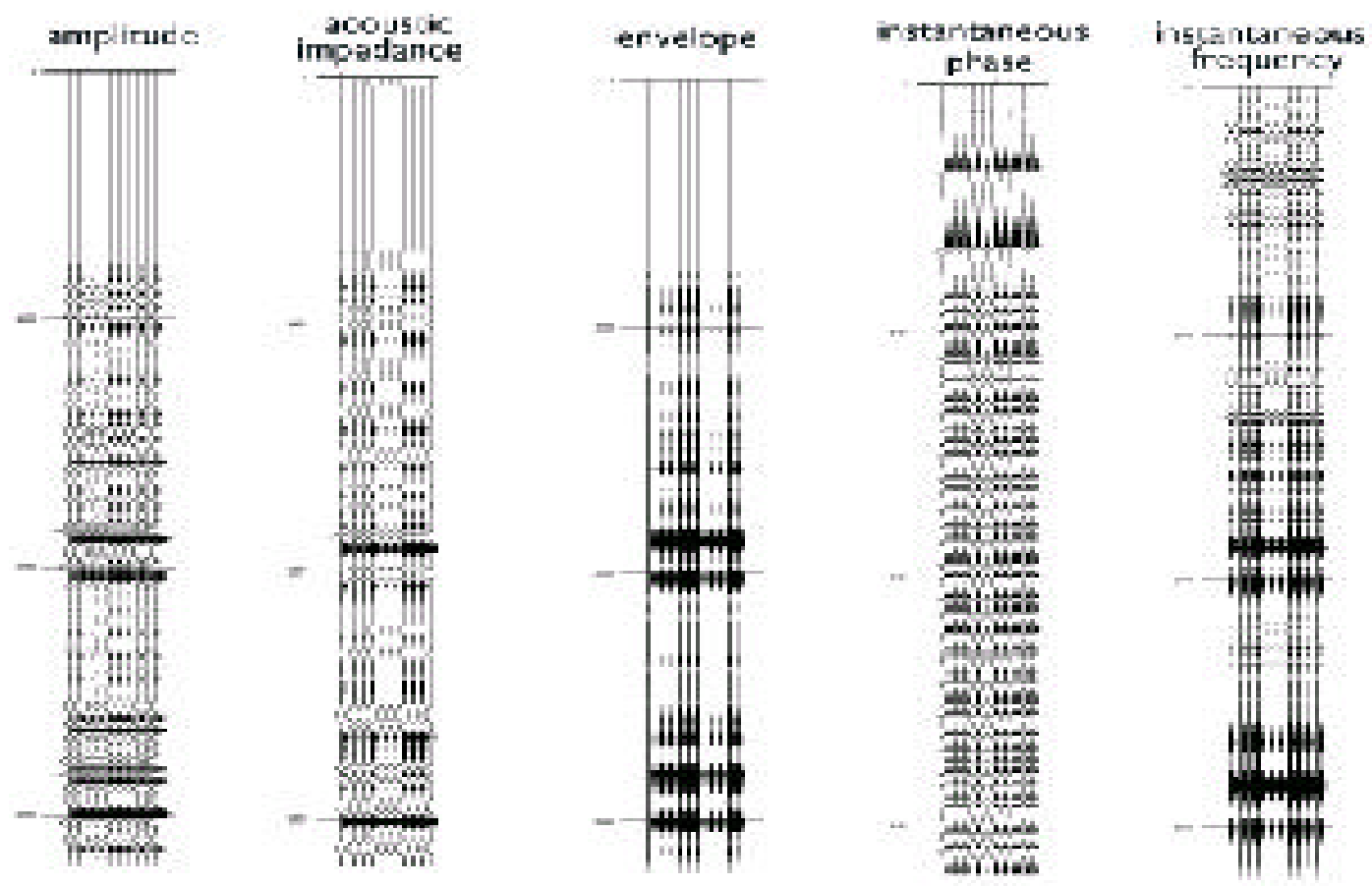

Figure 43 The synthetic traces for the CH2-CH4 line [13], repeated 10x

The next 56 traces (in the middle panel)- Appendix A- are the migrated crosswell reflections. On the $\mathrm{CH} 4-\mathrm{CH} 2$ plots, sequential trace number 2 corresponds to the location of $\mathrm{CH} 2$, and sequential trace 55 corresponds to the location of $\mathrm{CH} 4$. On the $\mathrm{CH} 1-\mathrm{CH} 3$ plots, trace 2 is at $\mathrm{CH} 1$ while trace 53 is at $\mathrm{CH} 3$.

Trace spacing is 2 meters, and sample interval is 0.5 meters.

The third panel- Appendix A- contains the 56 traces from the migrated surface data that correspond to the same subsurface reflection points as the crosswell traces. 
The Appendix A contains plotted all graphical representation described above. It contains 10 plots, each of them having those traces explained above in three panels:

- 10 traces for the synthetic trace $\left(1^{\text {st }}\right.$ panel $)$

- 56 traces for the migrated crosswell reflections $\left(2^{\text {nd }}\right.$ panel $)$

- 56 traces from the migrated surface data $\left(3^{\text {rd }}\right.$ panel $)$

In the Appendix A:

- Figures A1-A5: $\quad \mathrm{CH} 2$ is at trace 2; $\mathrm{CH} 4$ is at trace 55.

- Figures A6-A10: $\mathrm{CH} 1$ is at trace 2; $\mathrm{CH} 3$ is at trace 53.

They are 10 plots and 10 text files because:

For $\mathrm{CH} 1-\mathrm{CH} 3$ they are 5 plots/text files available

1. 1 plot/text file for the acoustic impedance for surface seismic and crosswell seismic

2. 1 plot/text file for the trace amplitudes for surface seismic and crosswell seismic

3. 1 plot/text file for the instantaneous phase for surface seismic and crosswell seismic

4. 1 plot/text file for the instantaneous frequency for surface seismic and crosswell seismic

5. 1 plot/text file for the trace envelope for surface seismic and crosswell seismic

The same 5 plots/text files was created for $\mathrm{CH} 2-\mathrm{CH} 4$ line. 


\section{IV.8.2 The text form}

There are 10 text files. In them the values are read from left to the right. There are 10 columns (Figure 44), the order of the values being:

- Values 1, 2, 3... 10 belongs to the $1^{\text {st }}$ row

- Values $11,12,13 \ldots 20$ belongs to the $2^{\text {nd }}$ row $\ldots$

\begin{tabular}{|c|c|c|c|c|c|c|c|c|c|c|}
\hline \multicolumn{11}{|c|}{ 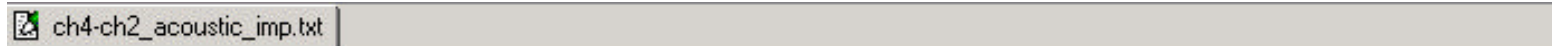 } \\
\hline \multicolumn{11}{|c|}{ 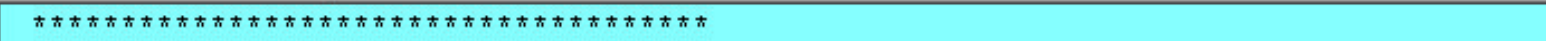 } \\
\hline \multicolumn{11}{|c|}{ 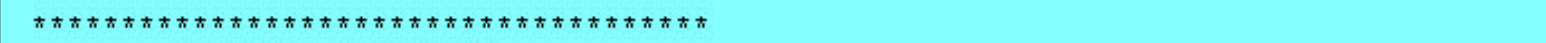 } \\
\hline \multicolumn{11}{|c|}{ ACOUSTIC IMPEDANCE } \\
\hline \multirow{2}{*}{\multicolumn{11}{|c|}{$\begin{array}{l}\text { Running sum of trace amplitudes } \\
\text { plus } 5 \mathrm{hz} \text {. low-cut filter }\end{array}$}} \\
\hline & & & & & & & & & & \\
\hline \multicolumn{11}{|c|}{ 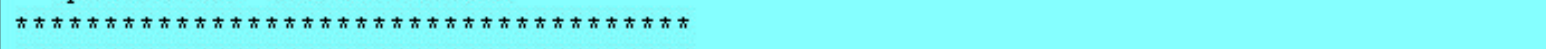 } \\
\hline \multicolumn{11}{|c|}{ 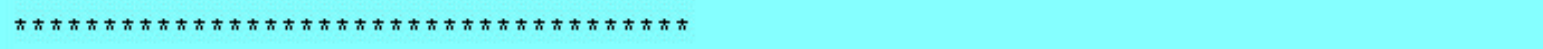 } \\
\hline \multicolumn{11}{|c|}{$\begin{array}{l}\text { File 1: synthetic traces from IN1 sonic log } \\
\text { Ten identical traces }\end{array}$} \\
\hline \multicolumn{11}{|c|}{ Sample interval: $\quad 0.5$ meters } \\
\hline \multicolumn{11}{|c|}{ Number of samples per trace: 641} \\
\hline \multicolumn{11}{|c|}{ Band pass filter: $20-40-150-300$} \\
\hline row 1 & -305 & -669 & -649 & -504 & -488 & -546 & -527 & -455 & -438 & -463 \\
\hline row 2 & -444 & -392 & -377 & -389 & -371 & -330 & -315 & -322 & -305 & -270 \\
\hline$\cdots$ & & & & & & & & & & \\
\hline row 64 & -10 & 793 & 1121 & 1119 & 979 & 851 & 798 & 800 & 799 & 768 \\
\hline row 65 & 723 & & & & & & & & & \\
\hline row 66 & -305 & -669 & -649 & -504 & -488 & -546 & -527 & -455 & -438 & -463 \\
\hline
\end{tabular}

Figure 44 The format of the text files available for the project

Each trace contains 641 values for a certain seismic attribute, from 0 to $320 \mathrm{~m}$, sampled at 0.5 meters. Because there are 10 values on each row, each text file contains 65 rows for each seismic trace (the last row contains only one value)

On each plot are represented 10 (synthetic trace) +56 (migrated crosswell reflections) +56 (migrated surface data $)=122$ traces; one text file has a header and 7930 rows containing the seismic attributes values $(122 * 65)$. The structure of the text file is:

- Rows 1-650 for synthetic trace 
- Rows 651-4290 for migrated crosswell reflections

- Rows 4291-7930 for migrated surface data

A computer program was written to convert this format. After conversion the data was arranged in 122 columns (one column for one trace) and 641 rows (because there are 641 depth values)

This format is suitable for Artificial Intelligence programs and is much closer to the human logic.

There was chosen a system having the origin $(0,0)$ value in the position of the well $\mathrm{CH} 2$.

Later the traces were arranged in one Excel file containing the trace number, the $\mathrm{x}, \mathrm{y}$ and $\mathrm{z}$ values, the surface seismic attributes and the crosswell seismic attributes- Table 2.

\begin{tabular}{|c|c|c|c|c|c|c|c|c|c|c|c|c|c|}
\hline- & \multicolumn{8}{|c|}{ INPUT } & \multicolumn{5}{|c|}{ OUTPUT } \\
\hline & & & & \multicolumn{5}{|c|}{ SURFACE SEISMIC } & \multicolumn{5}{|c|}{ CROSSWELL SEISMIC } \\
\hline trace & $\mathrm{z}$ & $\mathrm{x}$ & $\mathrm{y}$ & 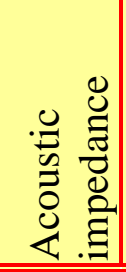 & 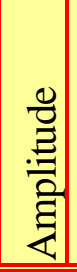 & 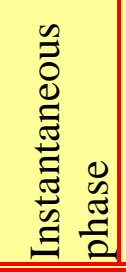 & 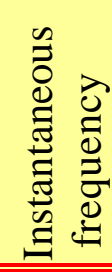 & 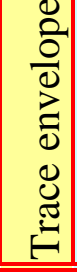 & 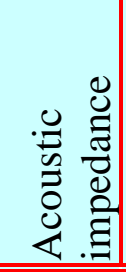 & 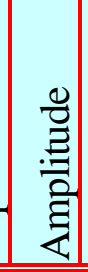 & 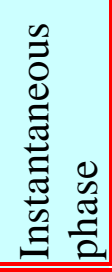 & 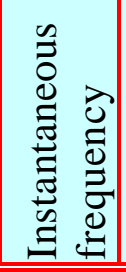 & 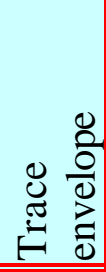 \\
\hline 28 & 110 & 36.8 & 36.8 & -1431 & 41 & -1299 & 572 & 174 & -31 & -8 & -2216 & -385 & 41 \\
\hline 28 & 110.5 & 36.8 & 36.8 & -1379 & 54 & -1232 & 813 & 178 & -31 & 1 & 1580 & -834 & 55 \\
\hline \multicolumn{14}{|c|}{$\ldots$} \\
\hline 28 & 299.5 & 36.8 & 36.8 & 5663 & 83 & 1346 & 220 & 397 & -10123 & 277 & -1463 & 2029 & 2566 \\
\hline 28 & 300 & 36.8 & 36.8 & 5743 & 79 & 1364 & -74 & 413 & -9217 & 915 & -1209 & 2047 & 2586 \\
\hline 29 & 110 & 38.2 & 38.2 & -2107 & 41 & -1365 & 322 & 230 & -70 & -43 & -2628 & -1128 & 59 \\
\hline 29 & 110.5 & 38.2 & 38.2 & -2059 & 51 & -1331 & 490 & 240 & -100 & -29 & 2699 & -1434 & 55 \\
\hline 29 & 111 & 38.2 & 38.2 & -1982 & 74 & -1253 & 727 & 253 & -149 & $|-50|$ & 2292 & -1542 & 83 \\
\hline
\end{tabular}

Table 2 The Excel file used in the mapping surface- crosswell seismic process 


\section{V.9 LOGS AVAI LABLE}

The log data regarding this field, as well as the interval available for the analysis (for each $\log$ ), is listed in Table 3.

\begin{tabular}{|c|c|c|c|c|c|c|c|c|c|c|c|}
\hline \multirow[t]{2}{*}{ Well } & & \multicolumn{2}{|c|}{$\begin{array}{l}\text { Sonic velocity } \\
\text { log }\end{array}$} & \multicolumn{2}{|c|}{ Density log } & \multicolumn{2}{|c|}{ Resistivity log } & \multicolumn{2}{|c|}{$\begin{array}{c}\text { Caliper } \\
\text { measurement }\end{array}$} & \multicolumn{2}{|c|}{$\begin{array}{c}\text { Gamma ray } \\
\text { log }\end{array}$} \\
\hline & & Start & End & Start & End & Start & End & Start & End & Start & End \\
\hline \multirow[t]{2}{*}{$\mathrm{CH} 1$} & & 80.2 & 299.96 & 80.2 & 299.656 & 80.2 & 299.96 & 80.2 & 299.656 & 80.2 & 299.96 \\
\hline & Records & \multicolumn{2}{|c|}{722} & \multicolumn{2}{|c|}{721} & \multicolumn{2}{|c|}{722} & \multicolumn{2}{|c|}{721} & \multicolumn{2}{|c|}{722} \\
\hline \multirow[t]{2}{*}{$\mathrm{CH} 2$} & & 80.2 & 299.66 & 80.2 & 229.85 & 80.2 & 299.35 & 80.2 & 299.96 & 80.2 & 299.96 \\
\hline & Records & \multicolumn{2}{|c|}{721} & \multicolumn{2}{|c|}{492} & \multicolumn{2}{|c|}{720} & \multicolumn{2}{|c|}{722} & \multicolumn{2}{|c|}{722} \\
\hline \multirow[t]{2}{*}{$\mathrm{CH} 3$} & & 80.2 & 298.74 & 80.2 & 299.04 & 80.2 & 299.656 & 80.2 & 299.96 & 80.2 & 299.96 \\
\hline & Records & \multicolumn{2}{|c|}{718} & \multicolumn{2}{|c|}{719} & \multicolumn{2}{|c|}{721} & \multicolumn{2}{|c|}{722} & \multicolumn{2}{|c|}{722} \\
\hline \multirow[t]{2}{*}{$\mathrm{CH} 4$} & & 80.2 & 299.96 & 80.2 & 299.656 & 80.2 & 299.96 & 80.2 & 299.656 & 80.2 & 299.656 \\
\hline & Records & \multicolumn{2}{|c|}{722} & \multicolumn{2}{|c|}{721} & \multicolumn{2}{|c|}{722} & \multicolumn{2}{|c|}{721} & \multicolumn{2}{|c|}{721} \\
\hline \multirow[t]{2}{*}{$\mathbb{N N 1}$} & & 204.2 & 324.9 & 204.2 & 318.195 & 204.2 & 325.2 & 204.2 & 323.68 & - & - \\
\hline & Records & \multicolumn{2}{|c|}{397} & \multicolumn{2}{|c|}{375} & \multicolumn{2}{|c|}{398} & \multicolumn{2}{|c|}{393} & \multicolumn{2}{|c|}{ - } \\
\hline
\end{tabular}

Table 3 Logs and depth intervals available for the Steepbank field

Because the sampling interval was not exactly at the points where surface seismic and crosswell seismic attributes were calculated, for eventually further calculations, the log values at those depths were computed by linear interpolation. 


\section{IV.10 THE RESOLUTI ON OF THE CROSSWELL SEI SMIC AND THE SURFACE SEI SMI C EXPERI MENTS}

The Appendix B shows, for $\mathrm{CH} 1-\mathrm{CH} 3$, the data spectra for the surface seismic and for the crosswell seismic. This spectra are also representative of the $\mathrm{CH} 2-\mathrm{CH} 4$ sections.

The spectra are a measure of the resolving capacity of the two datasets, and by resolving capacity we mean the ability of the data to distinguish closely spaced reflectors. In a sense one can think of the reflection waveforms representing some averaging process on the reflecting interfaces, and the wavelengths are a measure of the averaging interval. The averaging interval for the crosswell data is about one-half that of the surface data.

Significant signal frequencies in the crosswell data extend out to about $200 \mathrm{~Hz}$, , or 5 meters. The dominant frequency and wavelength in the data are about $120 \mathrm{~Hz}$. and 8 meters respectively.

The dominant frequency and wavelength on the surface data are about $60 \mathrm{~Hz}$. and 16 meters respectively, meaning that the minimum discernible reflector spacing is 4 meters, twice that of the crosswell data.

The spectra represent the average of the 10 traces shown on the left side of each plot. The vertical scale is linear, and it appears that the spectral bandwidth of the crosswell data is about twice that of the surface data.

The shape of the surface seismic attributes (used as input in the Artificial Neural Networks) are plotted together with the crosswell seismic attributes to be predicted (used as output in the Artificial Neural Networks) in the Appendix C. 


\section{IV.11 CHOI CE OF THE ZONE TO BE ANALYZED}

In the crosswell seismic, the many traces (and portions of traces) with zero amplitude represent the area between the two wells that received no reflections. This is a consequence of the crosswell survey's limited shot and geophone depth range.

Because of that, we had to choose the area of the field where both crosswell and surface seismic data are available. We considered that the depth range of the analysis is very important and we tried to find an optimum combination between the depth range and the number of traces available for the analysis. Increasing the depth range the number of traces will decrease in crosswell seismic - Figure 45 (the acoustic impedance was plotted here). For surface seismic the values are available for the entire zone surrounding the source well and the receiver well.

For this reason we have taken the following common area, where the training, calibration and verification traces exist (both for $\mathrm{CH} 2-\mathrm{CH} 4$ and $\mathrm{CH} 1-\mathrm{CH} 3$ ):

- Traces from trace 16 to trace 41

- Depth from $110 \mathrm{~m}$ to $300 \mathrm{~m}$ 


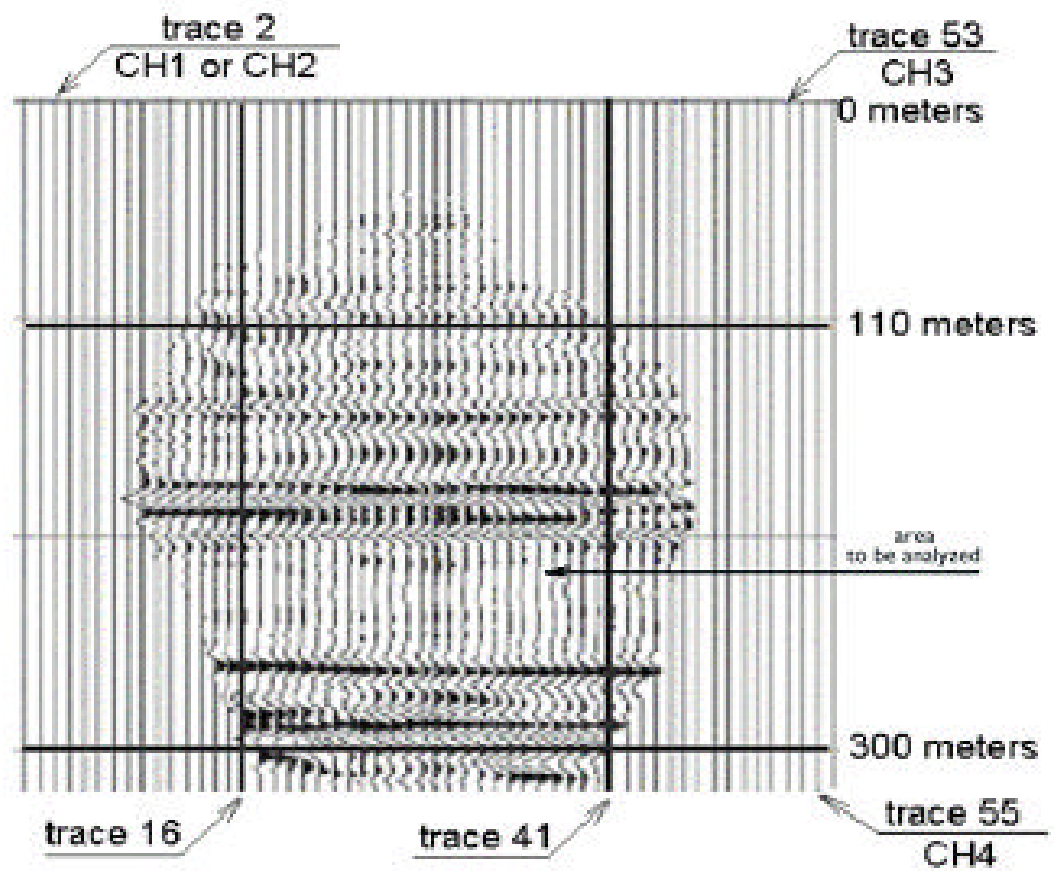

Figure 45 The area taken for the mapping surface seismic- crosswell seismic 


\section{CHAPTER V - RESULTS}

\section{V.1 CHARACTERI ZATI ON OF THE I NTERWELL SONIC VELOCI TI ES USI NG FI RST-TI ME ARRI VALS}

The computer tomography can give the sonic velocities based on the first time arrival traveltimes.

In the USBM (United States Bureau Of Mines) software were written for crosswell seismic interpretation. They were BOMTOM [56], MIGRATOM [20] and 3DTOM [21]. They were developed by Michael J. Jackson and Daryl R. Tweeton. Because they failed to run for the maximum number of travel times available for this project- 6093, Dr. Daryl Tweeton offered to us an improved version, called Geotom3D [57].

Geotom3D calculates the spatial distribution of physical properties in a rock mass, using measured characteristics of wave energy (seismic or electromagnetic) that has traveled though the rock.

There were 6093 travel times for the experiment with the wells CH1-CH3 and 5982 travel times for $\mathrm{CH} 2-\mathrm{CH} 4$ experiment.

Based on an initial guess (Geotom3D and other similar programs are very sensible to this guess) the program can compute the sonic velocities in the area surrounded by the source/receiver wells. Unfortunately the program crashed when we tried to use curved raypaths. The model obtained using straight rays seems to be unrealistic. We abandoned this approach of field definition because this method of interpreting the crosswell seismic experiments is known to give a non-unique solution. In our project we obtain an X shape result, as demonstrated in Figure 47.

The shape of the sonic log is not similar with the sonic logs measured in wells. The Figure 46 gives the results for the well $\mathrm{CH} 3$ after 400 runs, straight rays (the $\mathrm{CH} 1-\mathrm{CH} 3$ experiment). The range of the depth analyzed is $150-330$ meters. 
A plot of the zone surrounding the wells $\mathrm{CH} 1-\mathrm{CH} 3$, as obtained from the mathematical inversion, showed a mirror characteristic, most probably false (the values in Figure 47 was obtained after a kriging was performed, for a better resolution and in order to smooth the peaks observed in the Figure 46).

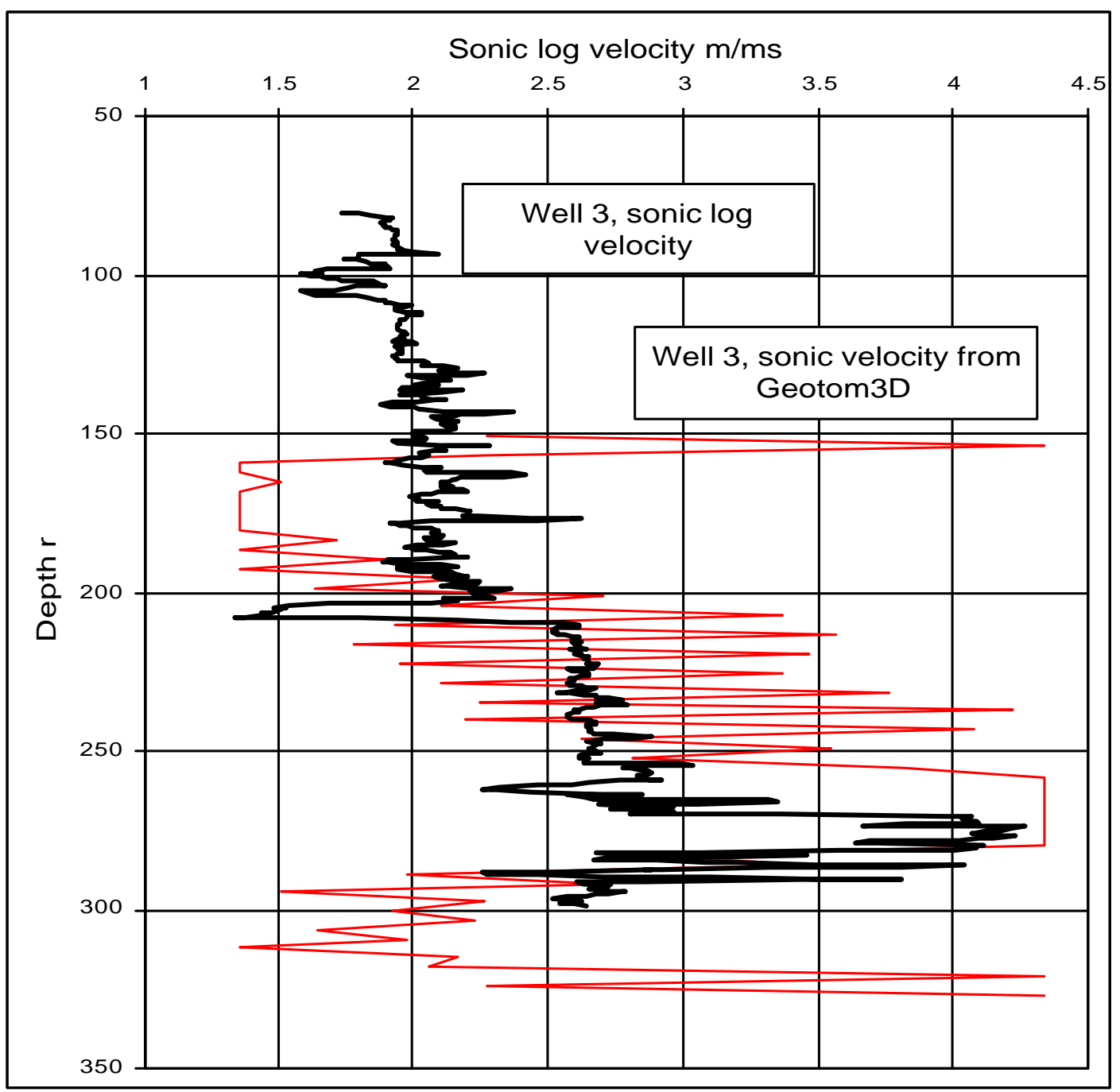

Figure 46 The shape of the sonic log obtained from Geotom3D compared with the real values- well $\mathrm{CH3}$ 


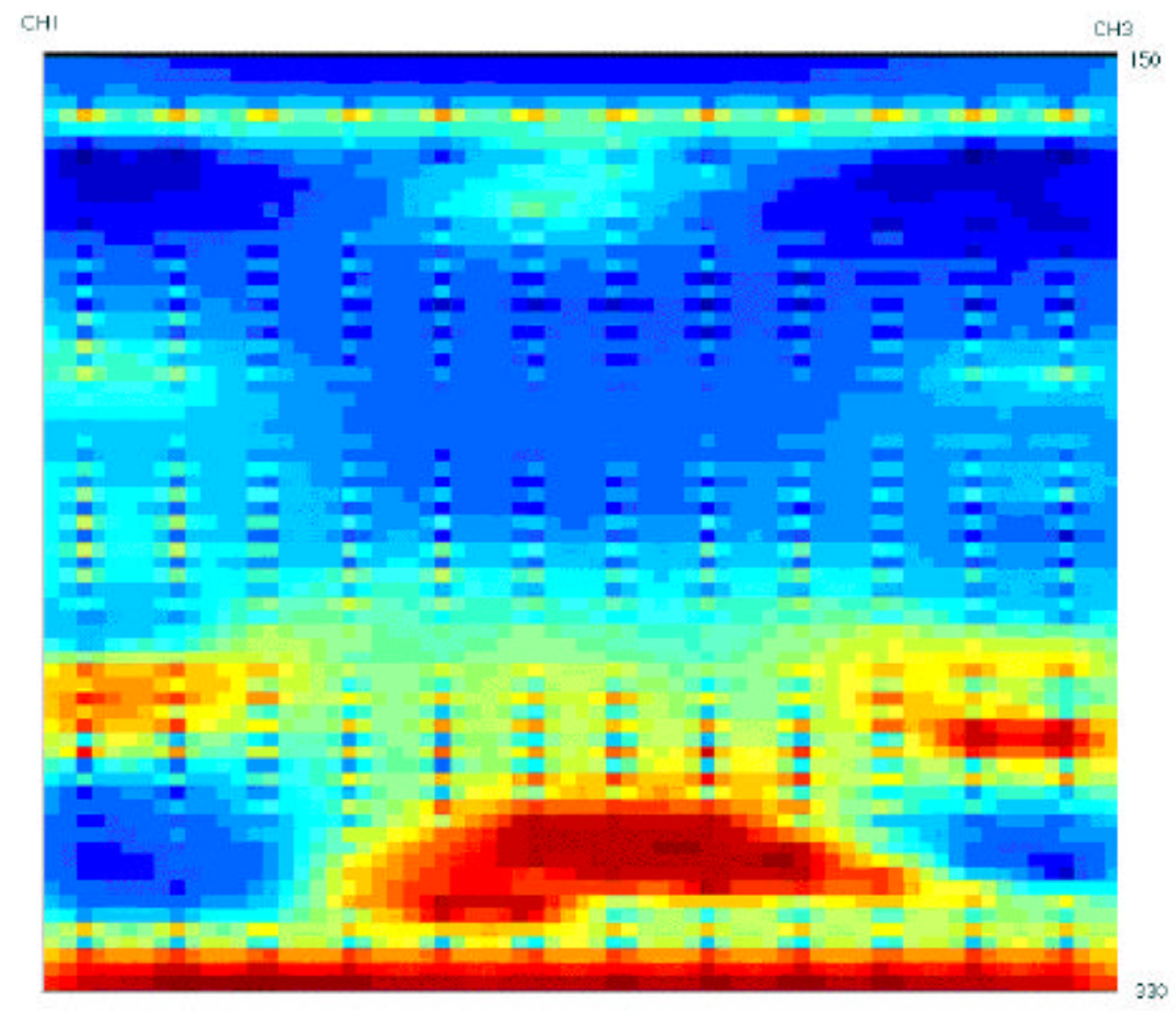

Figure 47 GEOTOM3D model for CH1-CH3 area

Unfortunately, traveltime tomography does not always provide sufficient resolution of the interwell lithology. The partial reason is that the set of traveltime equations resulting from the field acquisitions is underdetermined: i.e., there are not enough independent traveltime data to robustly solve for the many unknown parameters in the velocity models [59]. Many cells in the model are not intersected by the rays. 


\section{V.2 THE MAPPI NG CROSSWELL SEI SMI C- SURFACE SEI SMI C}

\section{V.2.1The input values}

For our mapping purposes we have two perpendicular experiments:

- One line from $\mathrm{CH} 1$ to $\mathrm{CH} 3$

- One line from $\mathrm{CH} 2$ to $\mathrm{CH} 4$

For each of them there were 26 traces available and we have information for surface seismic and crosswell seismic. In total we have 52 traces for surface seismic and 52 traces for crosswell seismic.

Each trace contains information from $110 \mathrm{~m}$ to $300 \mathrm{~m}$. They are sampled each 0.5 meters, each trace containing 381 measurements in this depth interval.

The traces are numbered in the following fashion:

- For the line $\mathrm{CH} 1$ to $\mathrm{CH} 3$ the trace 2 is closer to the well $\mathrm{CH} 1$; trace 16 is also closer to the well CH1

- For the line $\mathrm{CH} 2$ to $\mathrm{CH} 4$ the trace 2 is closer to the well $\mathrm{CH} 2$ trace 16 is also closer to the well $\mathrm{CH} 2$ - Figure 48 


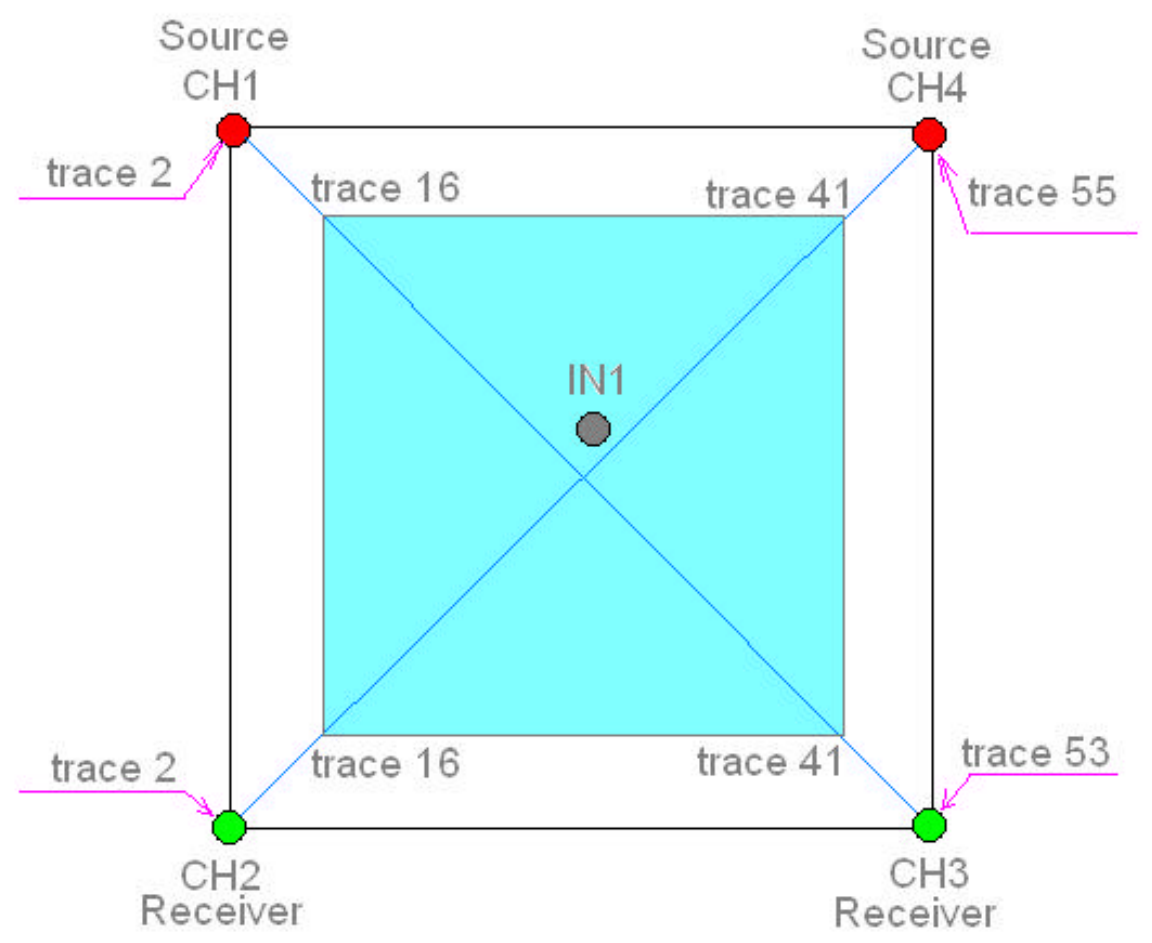

Figure 48 The well position and the traces notation for the project

The spatial arrangement of the wells and experiments performed are shown in Figure 49 and Figure 50. 


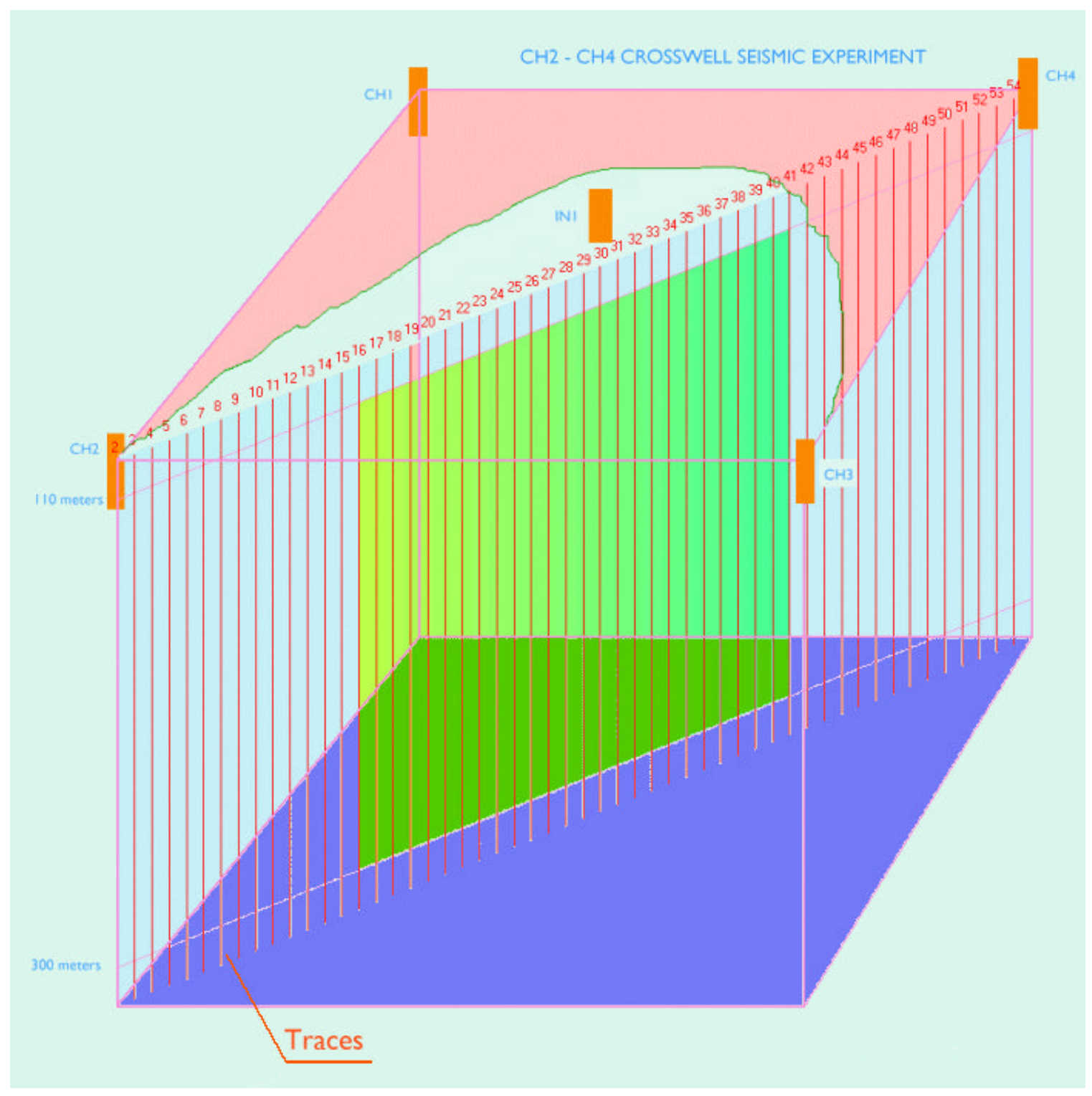

Figure 49 The $\mathrm{CH} 2-\mathrm{CH} 4$ crosswell seismic experiment 


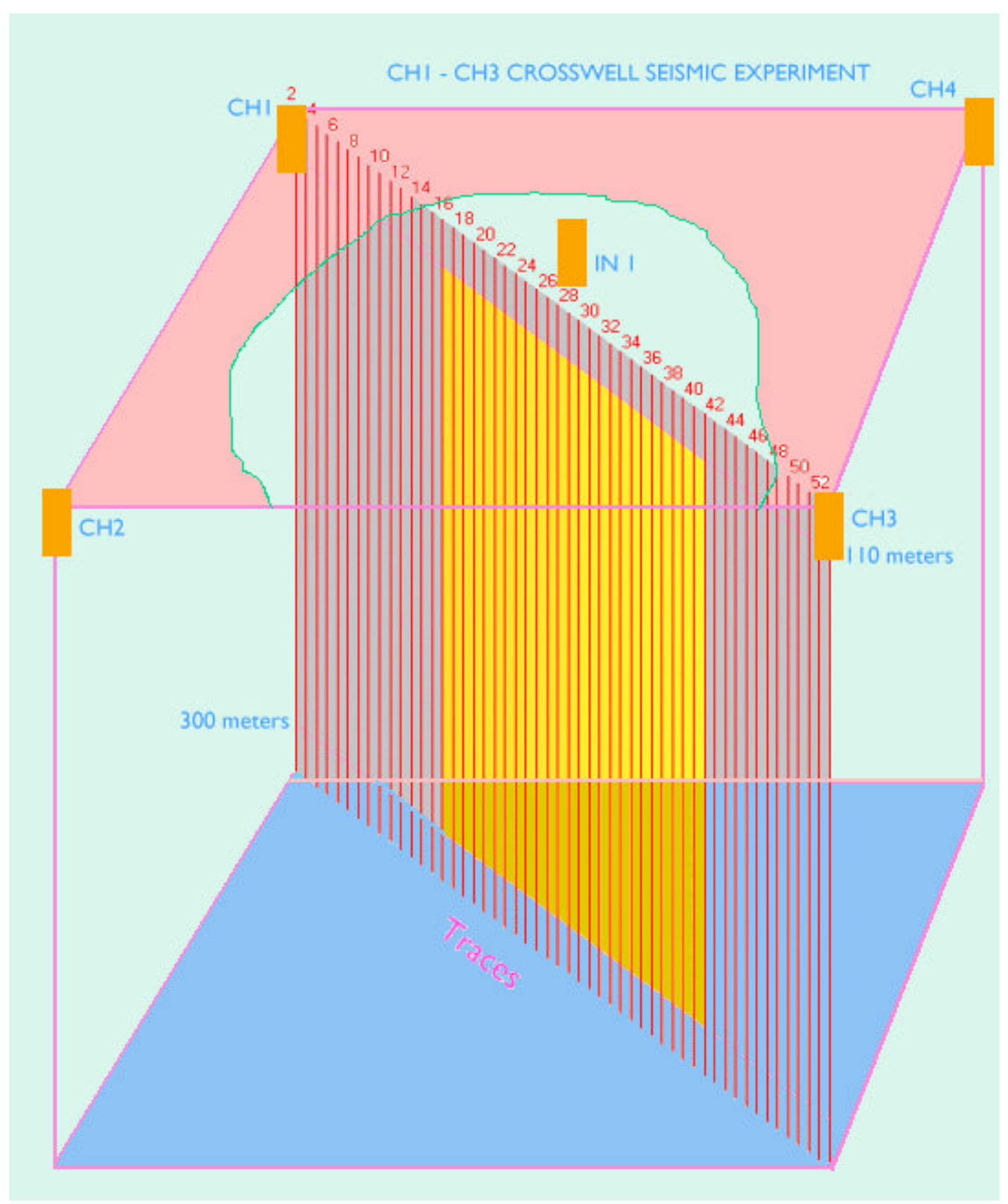

Figure 50 The CH1- CH3 crosswell seismic experiment 
Appendix $\mathrm{C}$ shows the shapes of the crosswell seismic traces and surface seismic traces. Our goal is to predict the former from the later.

We analyzed 20 patterns having in mind two major goals:

1. To find the best network

2. To show that the prediction is robust, i.e. to demonstrate that the choice of training, calibration and verification sets is not a major factor in prediction. We tried to show that the entire field can be predicted.

We used the General Regression Neural Network. For this network it was used:

$\underline{\text { As input }}: \mathrm{x}, \mathrm{y}, \mathrm{z}$ and surface seismic attributes

As output: crosswell seismic attributes

For example for the first network we used the following methodology (see the Table 4):

1. The training set was represented by 15240 cases representing 40 traces* 381 cases for each trace

2. The calibration set was represented by 3048 cases representing 8 traces* 381 cases for each trace

3. The verification set was represented by 1524 cases representing 4 traces* 381 cases for each trace

For example, the GRNN for the network 1 has the following neurons:

$\checkmark 8$ neurons in the input layer, for 8 input parameters (x,y,z and 5 surface seismic attributes)

$\checkmark 15250$ in the hidden layer, corresponding to the 15240 cases in the training set

$\checkmark 5$ neurons in the output layer, for 5 output parameters ( 5 crosswell seismic attributes) 


\section{V.2.2 Networks trained}

For the networks $1 \div 12,17$ and 18, each case consists of 8 features as input and 5 features as output. For the rest of the networks the number of input/output features was modified:

$\checkmark \quad$ In the network 13 it was used 5 more features representing the surface seismic features read at 0.5 meters above the current depth

$\checkmark$ In the network 14 it was used 10 more features representing the surface seismic features read at 0.5 meters an 1 meter above the current depth

$\checkmark$ In the network 15 it was used 15 more features representing the surface seismic features read at 0.5 meters an 1 meter above the current depth as well as the surface seismic features read at 0.5 meters below the current depth

$\checkmark$ In the network 16 it was used 20 more features representing the surface seismic features read at 0.5 meters an 1 meters above the current depth as well as the surface seismic features read at 0.5 and 1 meter below the current depth

$\checkmark$ In the network 19 it was used 7 features as input (the instantaneous phase was removed from the surface seismic input features) and the output contained four crosswell seismic attributes (the instantaneous phase was removed)

$\checkmark$ In the network 20 it was used all 8 features as input but the output contained four crosswell seismic attributes (the instantaneous phase was removed)

The traces used in training are: $18,19 \ldots 25,32,33 \ldots 41$ for the $\mathrm{CH} 1-\mathrm{CH} 3$ line and the same traces for the $\mathrm{CH} 2-\mathrm{CH} 4$ line. 
The input features are:

1. The $x$ coordinate of the trace

2. The y coordinate of the trace

3. The depth of the current case

4. Acoustic impedance

5. Conventional amplitudes

6. Instantaneous phase

7. Instantaneous frequency

8. Trace envelope

The output features are represented by the crosswell seismic attributes:

1. Acoustic impedance

2. Conventional amplitudes

3. Instantaneous phase

4. Instantaneous frequency

5. Trace envelope

The next table summarizes the choice of the training, calibration and verification traces. In our entire analysis we tried to predict entire traces from 110 to 300 meters:

- The regular cells represents the traces used in the training process

- The shaded cells represents the calibration traces

- The traces written in the bold format with border represents the verification traces 
$\operatorname{tr} 16 \operatorname{tr} 17 \operatorname{tr} 18 \operatorname{tr} 19 \operatorname{tr} 20 \operatorname{tr} 21 \quad \operatorname{tr} 22 \operatorname{tr} 23 \operatorname{tr} 24 \operatorname{tr} 25 \operatorname{tr} 26 \operatorname{tr} 27 \quad \operatorname{Tr} 28 \mid \operatorname{tr} 29 \operatorname{tr} 30 \operatorname{tr} 31 \operatorname{tr} 32 \operatorname{tr} 33 \operatorname{tr} 34 \operatorname{tr} 35 \operatorname{tr} 36 \operatorname{tr} 37 \operatorname{tr} 38 \operatorname{tr} 39 \operatorname{tr} 40 \operatorname{tr} 41$ $\operatorname{tr} 16 \operatorname{tr} 17 \operatorname{tr} 18 \operatorname{tr} 19 \operatorname{tr} 20$ tr21 $\operatorname{tr} 22 \operatorname{tr} 23 \operatorname{tr} 24 \operatorname{tr} 25 \operatorname{tr} 26 \quad \operatorname{tr} 27 \quad \operatorname{tr} 28 \operatorname{tr} 29 \operatorname{tr} 30 \operatorname{tr} 31 \operatorname{tr} 32 \operatorname{tr} 33 \operatorname{tr} 34 \operatorname{tr} 35 \operatorname{tr} 36 \operatorname{tr} 37 \operatorname{tr} 38 \operatorname{tr} 39 \operatorname{tr} 40 \operatorname{tr} 41$ $\operatorname{tr} 16 \operatorname{tr} 17 \operatorname{tr} 18 \operatorname{tr} 19 \operatorname{tr} 20$ tr21 $\operatorname{tr} 22 \operatorname{tr} 23 \operatorname{tr} 24 \operatorname{tr} 25 \operatorname{tr} 26 \operatorname{tr} 27 \operatorname{tr} 28 \operatorname{tr} 29 \operatorname{tr} 30 \quad \operatorname{tr} 31 \operatorname{tr} 32 \operatorname{tr} 33 \operatorname{tr} 34 \operatorname{tr} 35 \operatorname{tr} 36 \operatorname{tr} 37 \operatorname{tr} 38 \operatorname{tr} 39 \operatorname{tr} 40 \operatorname{tr} 41$ $\operatorname{tr} 16 \operatorname{tr} 17 \operatorname{tr} 18 \operatorname{tr} 19 \operatorname{tr} 20 \operatorname{tr} 21 \operatorname{tr} 22 \operatorname{tr} 33 \operatorname{tr} 24 \quad \operatorname{tr} 25 \operatorname{tr} 26 \operatorname{tr} 27 \operatorname{tr} 28 \operatorname{tr} 29 \operatorname{tr} 30 \operatorname{tr} 31 \operatorname{tr} 32 \operatorname{tr} 33 \operatorname{tr} 34 \operatorname{tr} 35 \operatorname{tr} 36 \operatorname{tr} 37 \operatorname{tr} 38 \operatorname{tr} 39 \operatorname{tr} 40 \operatorname{tr} 41$ $\operatorname{tr} 16 \operatorname{tr} 17 \operatorname{tr} 18 \operatorname{tr} 19 \operatorname{tr} 20 \operatorname{tr} 21 \operatorname{tr} 22 \operatorname{tr} 23 \quad \operatorname{Tr}_{24} \operatorname{tr} 25 \operatorname{tr} 26 \operatorname{tr} 27 \operatorname{tr} 28 \operatorname{tr} 29 \operatorname{tr} 30 \operatorname{tr} 31 \operatorname{tr} 32 \operatorname{tr} 33 \operatorname{tr} 34 \operatorname{tr} 35 \operatorname{tr} 36 \operatorname{tr} 37 \operatorname{tr} 38 \operatorname{tr} 39 \operatorname{tr} 40 \operatorname{tr} 41$ $\operatorname{tr} 16 \operatorname{tr} 17 \operatorname{tr} 18 \operatorname{tr} 19 \operatorname{tr} 20 \operatorname{tr} 21 \operatorname{tr} 22 \quad \operatorname{tr} 23 \operatorname{tr} 24 \operatorname{tr} 25 \operatorname{tr} 26 \operatorname{tr} 27 \operatorname{tr} 28 \operatorname{tr} 29 \operatorname{tr} 30 \operatorname{tr} 31 \operatorname{tr} 32 \operatorname{tr} 33 \operatorname{tr} 34 \operatorname{tr} 35 \operatorname{tr} 36 \operatorname{tr} 37 \operatorname{tr} 38 \operatorname{tr} 39 \operatorname{tr} 40 \operatorname{tr} 41$ $\operatorname{tr} 16 \operatorname{tr} 17 \operatorname{tr} 18 \operatorname{tr} 19 \operatorname{tr} 20 \operatorname{tr} 21 \quad \operatorname{tr} 22 \operatorname{tr} 23 \operatorname{tr} 24 \operatorname{tr} 25 \operatorname{tr} 26 \operatorname{tr} 27 \operatorname{tr} 28 \operatorname{tr} 29 \operatorname{tr} 30 \operatorname{tr} 31 \operatorname{tr} 32 \operatorname{tr} 33 \operatorname{tr} 34 \operatorname{tr} 35 \operatorname{tr} 36 \operatorname{tr} 37 \operatorname{tr} 38 \operatorname{tr} 39 \operatorname{tr} 40 \operatorname{tr} 41$ $\operatorname{tr} 16 \operatorname{tr} 17 \operatorname{tr} 18$ tr19 $\operatorname{tr} 20 \quad \operatorname{tr} 21 \operatorname{tr} 22 \operatorname{tr} 23 \operatorname{tr} 24 \quad \operatorname{tr} 25 \operatorname{tr} 26 \operatorname{tr} 27 \operatorname{tr} 28 \operatorname{tr} 29 \operatorname{tr} 30 \operatorname{tr} 31 \operatorname{tr} 32 \operatorname{tr} 33 \operatorname{tr} 34 \operatorname{tr} 35 \operatorname{tr} 36 \operatorname{tr} 37 \operatorname{tr} 38 \operatorname{tr} 39 \operatorname{tr} 40 \operatorname{tr} 41$ $\operatorname{tr} 16 \operatorname{tr} 17 \operatorname{tr} 18 \operatorname{tr} 19 \quad \operatorname{tr} 20 \quad \operatorname{tr} 21 \quad \operatorname{tr} 22 \operatorname{tr} 23 \operatorname{tr} 24 \quad \operatorname{tr} 25 \operatorname{tr} 26 \operatorname{tr} 27 \operatorname{tr} 28 \operatorname{tr} 29 \operatorname{tr} 30 \operatorname{tr} 31 \operatorname{tr} 32 \operatorname{tr} 33 \operatorname{tr} 34 \operatorname{tr} 35 \operatorname{tr} 36 \operatorname{tr} 37 \operatorname{tr} 38 \operatorname{tr} 39 \operatorname{tr} 40 \operatorname{tr} 41$

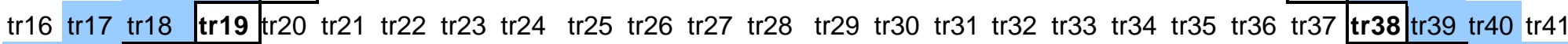
$\operatorname{tr} 16 \operatorname{tr} 17 \quad \operatorname{tr} 18 \quad \operatorname{tr} 19 \operatorname{tr} 20 \operatorname{tr} 21 \quad \operatorname{tr} 22 \operatorname{tr} 23 \operatorname{tr} 24 \operatorname{tr} 25 \operatorname{tr} 26 \operatorname{tr} 27 \operatorname{tr} 28 \operatorname{tr} 29 \operatorname{tr} 30 \operatorname{tr} 31 \operatorname{tr} 32 \operatorname{tr} 33 \operatorname{tr} 34 \operatorname{tr} 35 \operatorname{tr} 36 \operatorname{tr} 37 \operatorname{tr} 38 \operatorname{tr} 39 \operatorname{tr} 40 \operatorname{tr} 41$ $\operatorname{tr} 16 \operatorname{tr} 17 \operatorname{tr} 18 \quad \operatorname{tr} 19 \operatorname{tr} 20$ tr21 $\operatorname{tr} 22 \operatorname{tr} 23 \operatorname{tr} 24 \operatorname{tr} 25 \operatorname{tr} 26 \operatorname{tr} 27 \operatorname{tr} 28 \operatorname{tr} 29 \operatorname{tr} 30 \operatorname{tr} 31 \operatorname{tr} 32 \operatorname{tr} 33 \operatorname{tr} 34 \operatorname{tr} 35 \operatorname{tr} 36 \operatorname{tr} 37 \operatorname{tr} 38 \operatorname{tr} 39 \operatorname{tr} 40 \operatorname{tr} 41$ $\operatorname{tr} 16 \operatorname{tr} 17 \operatorname{tr} 18 \quad \operatorname{tr} 19 \operatorname{tr} 20$ tr21 tr22 $\operatorname{tr} 23 \operatorname{tr} 24$ tr25 tr26 tr27 tr28 $\operatorname{tr} 29 \operatorname{tr} 30 \operatorname{tr} 31 \operatorname{tr} 32 \operatorname{tr} 33 \operatorname{tr} 34 \operatorname{tr} 35 \operatorname{tr} 36 \operatorname{tr} 37 \operatorname{tr} 38 \operatorname{tr} 39 \operatorname{tr} 40 \operatorname{tr} 41$ $\operatorname{tr} 16 \operatorname{tr} 17 \operatorname{tr} 18 \quad \operatorname{tr} 19 \operatorname{tr} 20$ tr21 tr22 $\operatorname{tr} 23 \operatorname{tr} 24$ tr25 tr26 tr27 tr28 $\operatorname{tr} 29 \operatorname{tr} 30 \operatorname{tr} 31 \operatorname{tr} 32 \operatorname{tr} 33 \operatorname{tr} 34 \operatorname{tr} 35 \operatorname{tr} 36 \operatorname{tr} 37 \operatorname{tr} 38 \operatorname{tr} 39 \operatorname{tr} 40 \operatorname{tr} 41$ $\operatorname{tr} 16 \operatorname{tr} 17 \operatorname{tr} 18 \quad \operatorname{tr} 19 \operatorname{tr} 20$ tr2 1 tr22 $\operatorname{tr} 23 \operatorname{tr} 24 \operatorname{tr} 25 \operatorname{tr} 26 \operatorname{tr} 27 \operatorname{tr} 28 \operatorname{tr} 29 \operatorname{tr} 30 \operatorname{tr} 31 \operatorname{tr} 32 \operatorname{tr} 33 \operatorname{tr} 34 \operatorname{tr} 35 \operatorname{tr} 36 \operatorname{tr} 37 \operatorname{tr} 38 \operatorname{tr} 39 \operatorname{tr} 40 \operatorname{tr} 41$ $\operatorname{tr} 16 \operatorname{tr} 17 \operatorname{tr} 18 \quad \operatorname{tr} 19 \operatorname{tr} 20 \operatorname{tr} 21$ tr22 $\operatorname{tr} 23 \operatorname{tr} 24 \operatorname{tr} 25 \operatorname{tr} 26 \operatorname{tr} 27 \operatorname{tr} 28 \quad \operatorname{tr} 29 \operatorname{tr} 30 \operatorname{tr} 31 \operatorname{tr} 32 \operatorname{tr} 33 \operatorname{tr} 34 \operatorname{tr} 35 \operatorname{tr} 36 \operatorname{tr} 37 \operatorname{tr} 38 \operatorname{tr} 39 \operatorname{tr} 40 \operatorname{tr} 41$ $\operatorname{tr} 16 \operatorname{tr} 17 \operatorname{tr} 18 \operatorname{tr} 19 \operatorname{tr} 20 \operatorname{tr} 21 \quad \operatorname{tr} 22 \operatorname{tr} 23 \operatorname{tr} 24 \operatorname{tr} 25 \operatorname{tr} 26 \operatorname{tr} 27 \quad \operatorname{tr} 28 \mid \operatorname{tr} 29 \operatorname{tr} 30 \operatorname{tr} 31 \operatorname{tr} 32 \operatorname{tr} 33 \operatorname{tr} 34 \operatorname{tr} 35 \operatorname{tr} 36 \operatorname{tr} 37 \operatorname{tr} 38 \operatorname{tr} 39 \operatorname{tr} 40 \operatorname{tr} 41$ $\operatorname{tr} 16 \operatorname{tr} 17 \quad \operatorname{Tr} 18 \operatorname{tr} 19 \operatorname{tr} 20$ tr2 1 tr22 $\operatorname{tr} 23 \operatorname{tr} 24 \operatorname{tr} 25 \operatorname{tr} 26 \operatorname{tr} 27 \quad \operatorname{Tr} 28 \operatorname{tr} 29 \operatorname{tr} 30 \operatorname{tr} 31 \operatorname{tr} 32 \operatorname{tr} 33 \operatorname{tr} 34 \operatorname{tr} 35 \operatorname{tr} 36 \operatorname{tr} 37 \operatorname{tr} 38 \operatorname{tr} 39 \operatorname{tr} 40 \quad \operatorname{tr} 41$ $\operatorname{tr} 16 \operatorname{tr} 17 \operatorname{tr} 18 \quad \operatorname{tr} 19 \operatorname{tr} 20 \operatorname{tr} 21$ tr22 $\operatorname{tr} 23 \quad$ Tr24 tr25 tr26 tr27 $\operatorname{Tr} 28 \operatorname{tr} 29 \operatorname{tr} 30 \operatorname{tr} 31 \operatorname{tr} 32 \operatorname{tr} 33 \operatorname{tr} 34 \operatorname{tr} 35 \operatorname{tr} 36 \operatorname{tr} 37 \operatorname{tr} 38 \operatorname{tr} 39 \operatorname{tr} 40 \operatorname{tr} 41$ $\operatorname{tr} 16 \operatorname{tr} 17 \operatorname{tr} 18 \quad \operatorname{tr} 19 \operatorname{tr} 20$ tr21 tr22 tr23 Tr24 tr25 tr26 tr27 Tr28 tr29 $\operatorname{tr} 30 \operatorname{tr} 31 \operatorname{tr} 32 \operatorname{tr} 33 \operatorname{tr} 34 \operatorname{tr} 35 \operatorname{tr} 36 \operatorname{tr} 37 \operatorname{tr} 38 \operatorname{tr} 39 \operatorname{tr} 40 \operatorname{tr} 41$

* The same traces were taken for training and calibration sets in both lines: $\mathrm{CH} 1-\mathrm{CH} 3$ and $\mathrm{CH} 2-\mathrm{CH} 4$

for training $x, y, z$ and 5 surface seismic attributes, output 5 crosswell seismic attributes

trxx calibration trace trxx Training trace

trxx verification trace

(1) training set, seismic attributes at $z$ and at $z-0.5 \mathrm{~m}(\mathrm{x}, \mathrm{y}, \mathrm{z}$ and 10 seismic attributes), output 5 seismic attributes at $z$

(2) training set, seismic attributes at $z$, at $z-0.5 \mathrm{~m}$ and $z-1 \mathrm{~m}(x, y, z$ and 15 seismic attributes), output 5 seismic attributes at $z$

(3) training set, seismic attributes at $z$, at $z+0.5, z-0.5 \mathrm{~m}$ and $z-1 \mathrm{~m}(x, y, z$ and 20 seismic attributes), output 5 seismic attributes at $z$

(4) training set, seismic attributes at $z$, at $z+0.5, z+1, z-0.5 \mathrm{~m}$ and $z-1 \mathrm{~m}(\mathrm{x}, \mathrm{y}, \mathrm{z}$ and 25 seismic attributes), output 5 seismic attributes at $z$

(5) training set without instantaneous phase ( $x, y, z$ and 4 seismic attributes), try to predict 4 seismic attributes (without the instantaneous phase) at $z$

(6) training set complete $(x, y, z$ and 5 seismic attributes), try to predict 4 seismic attributes (without the instantaneous phase) at $z$

Table 4 The choice of traces and input/output in the ANN analyzed 


\section{V.2.3 Networks results}

The results of the run are presented in Appendix D. This appendix contains the major statistical comparison between the two data sets- the real $381 * 4$ values for the verification sets and the same number of values coming from the prediction.

All runs were performed using General Regression Neural Networks. In our analysis they showed better performance comparing to the backpropagation neural networks.

We used as input $\mathrm{x}, \mathrm{y}, \mathrm{z}$ and surface seismic attributes and tried to predict the crosswell seismic attributes.

The Appendix D tables provide the statistical comparison between the real and the predicted values for all the networks that were developed for this study. List of traces used for training, calibration and verification are provided in Table 4. In this table C1 through C5 represent the attributes as follows:

- $\mathrm{C} 1=$ Acoustic impedance

- $\mathrm{C} 2=$ Trace amplitude

- $\mathrm{C} 3=$ Instantaneous phase

- $\mathrm{C} 4=$ Instantaneous frequency

- C5= Envelope

In all the networks (except those identified in Table 4, where the additional input parameters are identified) $\mathrm{x}, \mathrm{y}, \mathrm{z}$ and surface seismic attributes are used as input and the abovementioned crosswell seismic attributes were the targeted outputs.

Figure 51, Figure 52 and Figure 53 show the change of the average R2 values as a function of the network numbers. The network numbers correspond to particular selection of training, calibration and verification datasets as was mentioned in the previous section. 


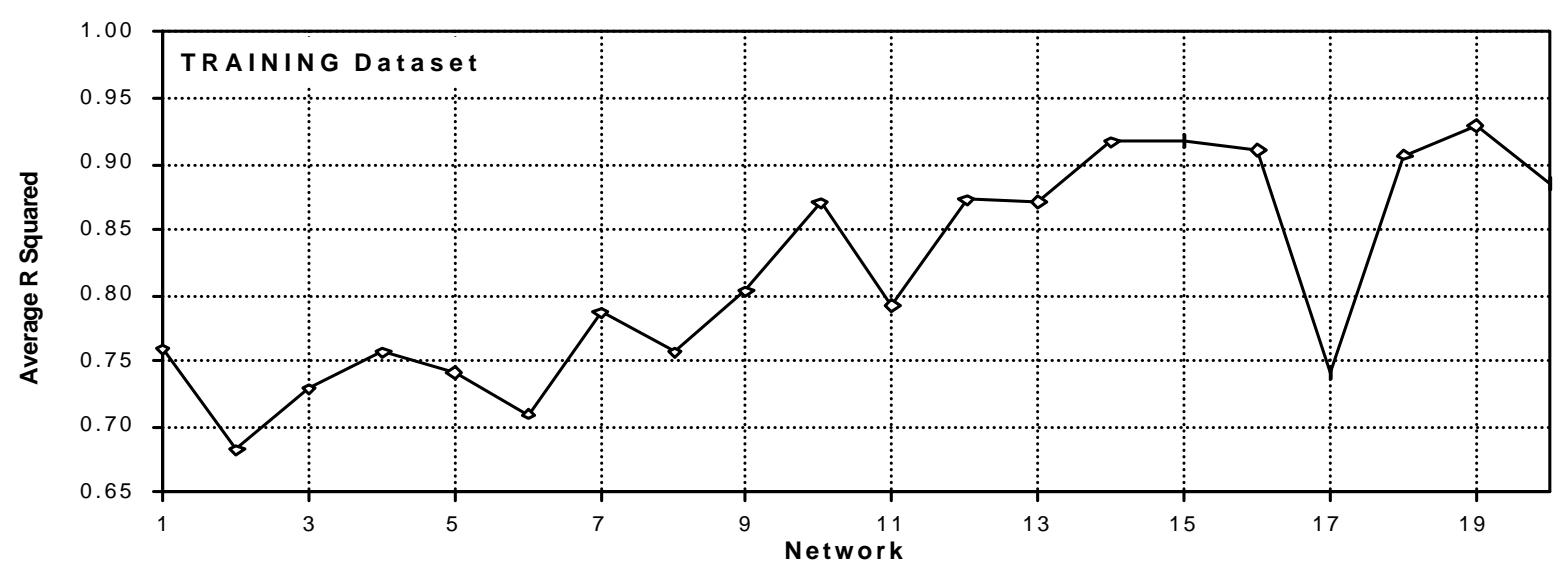

Figure 51 Average $\mathbf{R 2}$ of the training sets for different networks

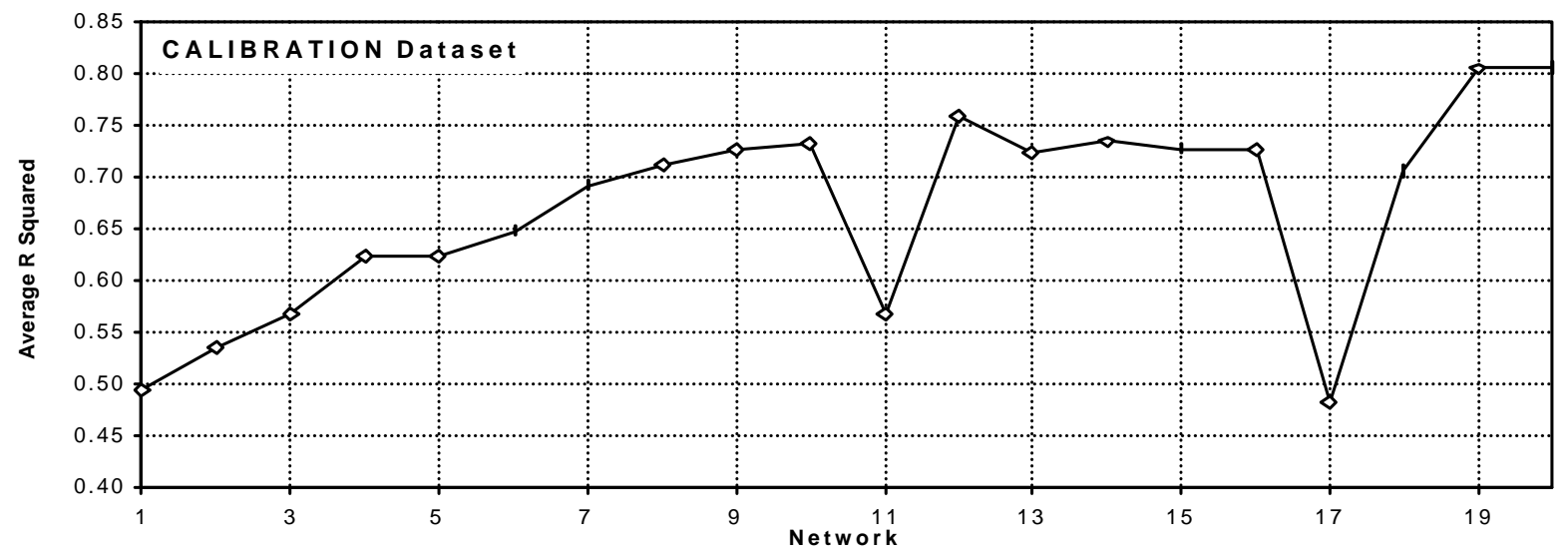

Figure 52 Average $\mathbf{R} 2$ of the calibration sets for different networks

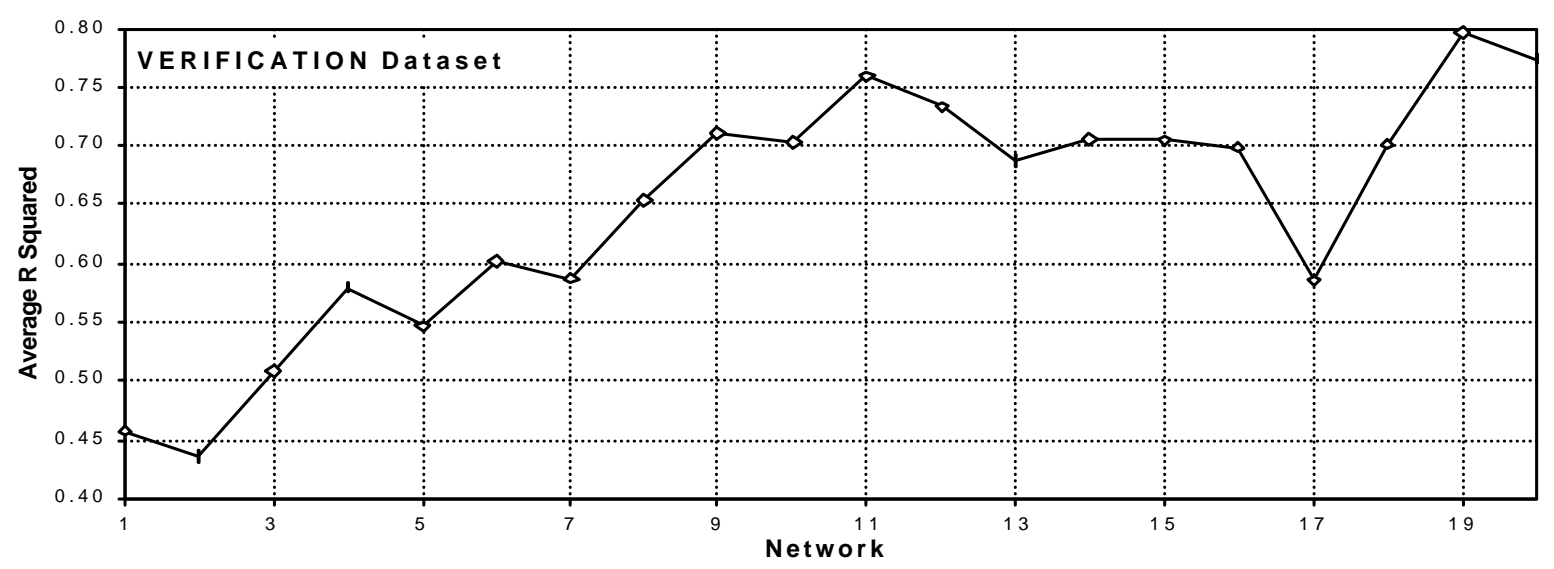

Figure 53 Average $\mathbf{R 2}$ of the verification sets for different networks 
The Artificial Neural Networks best predic ted the envelope. For the verification set, the statistical performance of the predictions, regarding this attribute, are:

- The minimum value for $\mathrm{R}^{2}$ is 0.667 (Network 1 ) and the minimum correlation coefficient is 0.8258 (Network 1)

- The maximum value for $\mathrm{R}^{2}$ is 0.9101 (Network 11) and the maximum correlation coefficient is 0.9557 (Network 11)

From Appendix D it can be concluded that the most difficult crosswell seismic to predict seems to be the instantaneous phase. By looking at the shape of this attribute and its sharp slope changes one can see why it was harder to predict than other attributes.

For the verification set, in the analysis of all seismic attributes, the statistical performance of the predictions are:

- The minimum value for $\mathrm{R}^{2}$ is 0.2287 (Network 2) and the minimum correlation coefficient is 0.48 (Network 2)

- The maximum value for $\mathrm{R}^{2}$ is 0.5261 (Network 18) and the maximum correlation coefficient is 0.9557 (Network 18)

The best predictions in the verification set for the envelope as well as for the instantaneous phase are presented in Figure 54. All the traces for the verification set can be found in the Appendix E. 

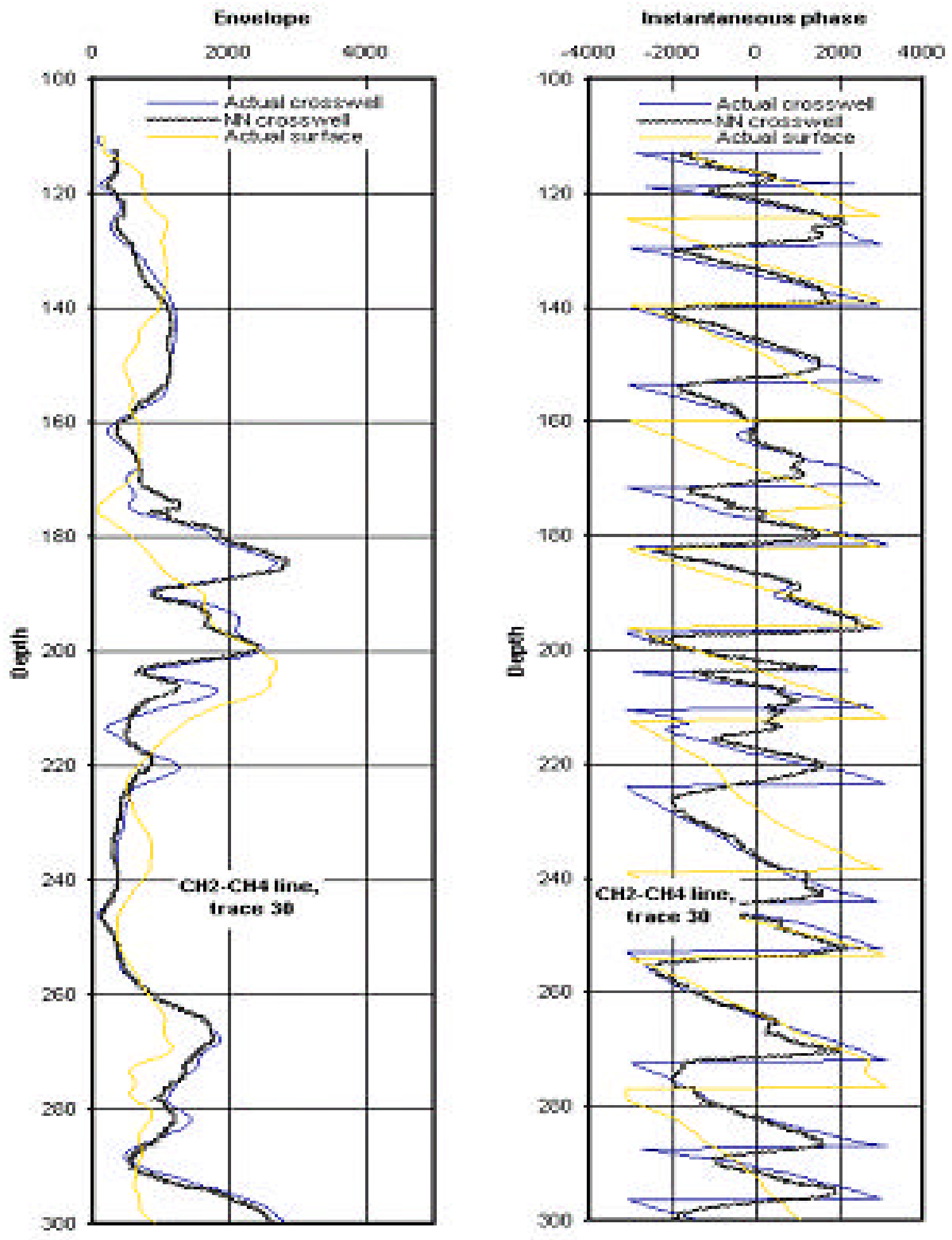

Figure 54 The network predictions for the crosswell seismic traces- the best prediction (left) for the envelope and the worst prediction (right) for the instantaneous phase- trace 30, CH2CH4 line 
The analysis shown that practically any crosswell seismic trace can be predicted with accuracy (the Appendix E). This is an important issue taking into account the frequency content of the traces- Appendix A. In the crosswell seismic, the dominant frequency and wavelength are about $120 \mathrm{~Hz}$. and 8 meters respectively. In the same time the dominant frequency and wavelength on the surface data are about $60 \mathrm{~Hz}$. and 16 meters respectively, meaning that the minimum discernible reflector spacing is 4 meters, twice that of the crosswell data [14].

We have shown that it is possible to predict a high resolution trace (crosswell seismic) using o low resolution trace (surface seismic).

It is important to note that all the statistical indicators $\left(\mathrm{R}^{2}, \mathrm{r}^{2}\right.$, the correlation coefficient) are positive for all the analysis sets- training, calibration, and verification. The shape for the average of the statistical parameters $(\mathrm{C} 1+\mathrm{C} 2+\mathrm{C} 3+\mathrm{C} 4+\mathrm{C} 5) / 5$ or $(\mathrm{C} 1+\mathrm{C} 2+\mathrm{C} 4+\mathrm{C} 5) / 4$ (depending of the network used) is depicted in Figure 55.

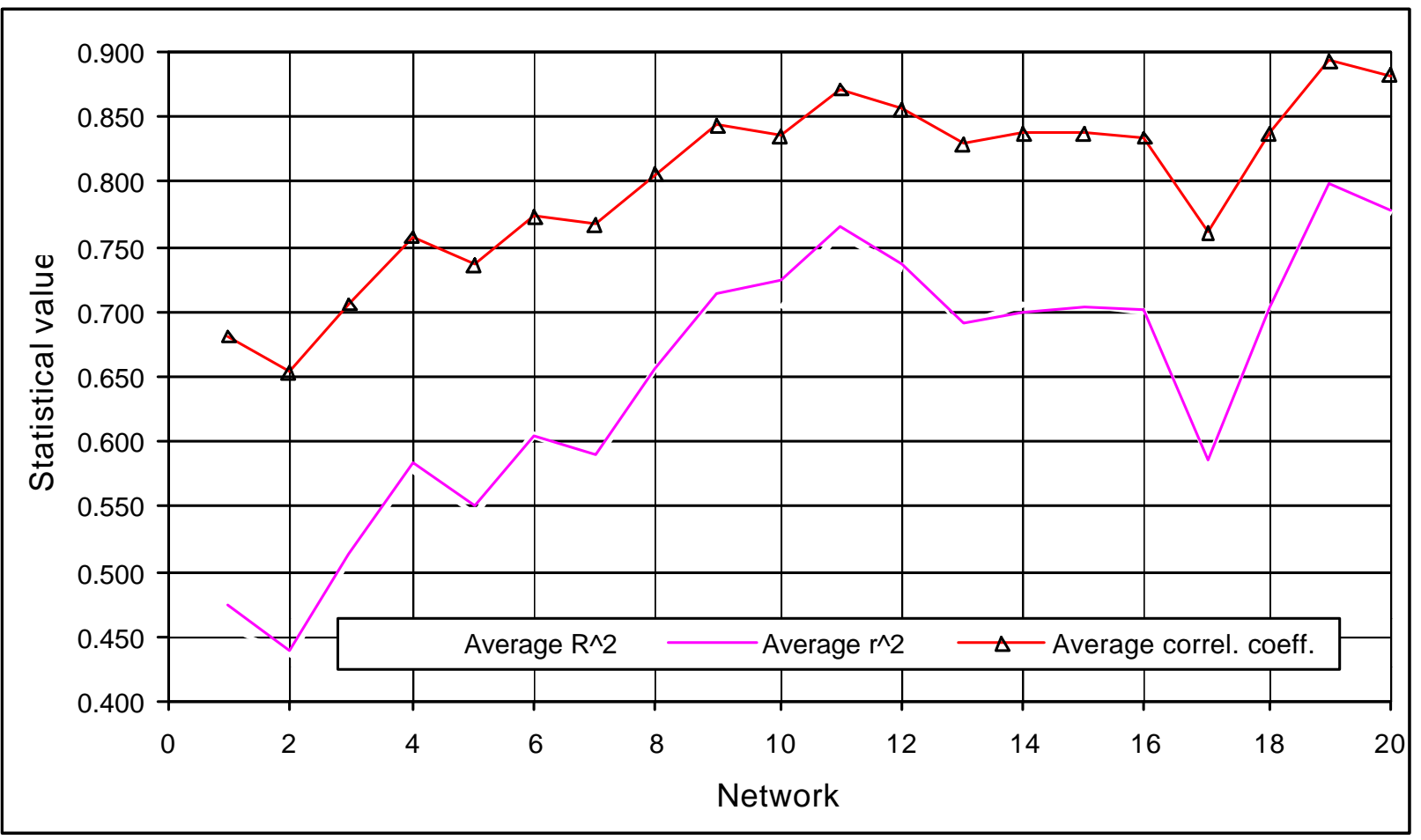

Figure 55 The average of the statistical indicators 
From Figure 55 and Appendix D it can be seen that the Artificial Neural Networks performance is better when the calibration/verification traces are either at the edge of the area analyzed (corresponding to an increase in the network index from 1 to 11) or scattered through the field (networks 17 and 18 analyzed)

For this field, better results are not obtained if in the analysis is included information about the trace shapes above/below the current depths. The networks $13 \div 16$ are similar with the network 12 (regarding the traces taken in the training/calibration/verification sets) but some information about the shape of the curves above/below the current depth was included. The prediction results do not show any improvement in predictions (Figure 55).

In our opinion the best network is the network 12. It shown an average better performance regarding the training/calibration/verification sets. It is true that for the verification set the best prediction were done by Artificial Neural Network number 11 but this one shown worse performance in the training and calibration sets.

All the seismic attributes are important in the training process. Exclusion of any seismic attributes in input/output decreases the accuracy of the prediction. Because in the networks 1-18 the instantaneous phase was the most difficult to predict, we tried to remove it from the input traces (surface seismic traces) and we tried to predict only four crosswell seismic traces- network 19.

Because in general we didn't notice a significant prediction improvement, in network 20, we tried to use all the surface seismic traces but to predict only four crosswell seismic traces. Even in this case the prediction performance was not improved. 


\section{CONCLUSIONS}

This project shown that:

1. In order to better defined the field, one of the latest technologies are represented by the crosswell seismic

2. The crosswell seismic has a better resolution compared to the surface seismic. In our project the minimum discernible reflector spacing of the surface data is twice the value of the crosswell data.

3. Comparing with the surface seismic, the crosswell seismic has four major disadvantages:

a. It must be performed with a greater care

b. The experiment is more expensive

c. The experiment covers only a limited area of the field

d. The method applied by Chevron to increase the accuracy (cementing the geophones in the annulus between the tubing and casing) can not be applied in the entire field

4. By now the method widely used for the crosswell seismic interpretation was the mathematical inversion technique known as tomography

5. Unlike the tomography used in medicine the method used in the petroleum industry has some limitations:

a. the layer to be analyzed can not be surrounded by sources

b. the number of sources and receivers is limited. Some cells can not be covered by rays

c. the zone covered by the experiment has not a constant velocity

d. due to the distance between sources and receivers the frequency content is limited

6. Due to the dimension of the field, a better description can be done with a large number of sources and receivers. Their number increases the cost of the experiment and increase the number of equations to be solved by computer. Anyway, increasing their number doesn't guaranty that the solution is unique. 
7. In computer tomography, a large number of equations can cause the crash of many commercial software. If the crash is not encountered, the running time can be too long. For our project the USBM software were unable to run for the given traveltimes (around 6000 traveltimes)

8. The literature mentioned the fact that the solution obtained by mathematical inversion can be non-unique

9. Even if an unique solution is obtained from mathematical inversion, the volume defined is some order of magnitudes smaller than the entire field volume

10. The surface seismic has a larger volume of coverage compared with the crosswell seismic.

11. A mapping surface seismic-crosswell seismic could give the chance to define the field with a greater resolution using the predictions of the surface seismic traces

12. One algorithm to deconvolve "low" resolution 3-D seismic data to a "high" resolution cross-well seismic or well $\log$ scale would take the advantage of all methods: the resolution of the crosswell seismic lie between the log/cores resolution and the surface seismic resolution

13. The algorithm could be the solution for an improved reservoir management by including the seismic experiments results in the field analysis

14. This project shown that the deconvolution 3-D seismic $\leftrightarrow$ cross-well seismic is possible

15. We had data from an experiment conducted by Chevron at Canada's Oil Sand Leas 49 (Steepbank)

16. The advantage of the Chevron experiment is that, for a 5- spot pattern, two crosswell seismic experiments directions are available as well as the surface seismic data for the same lines 


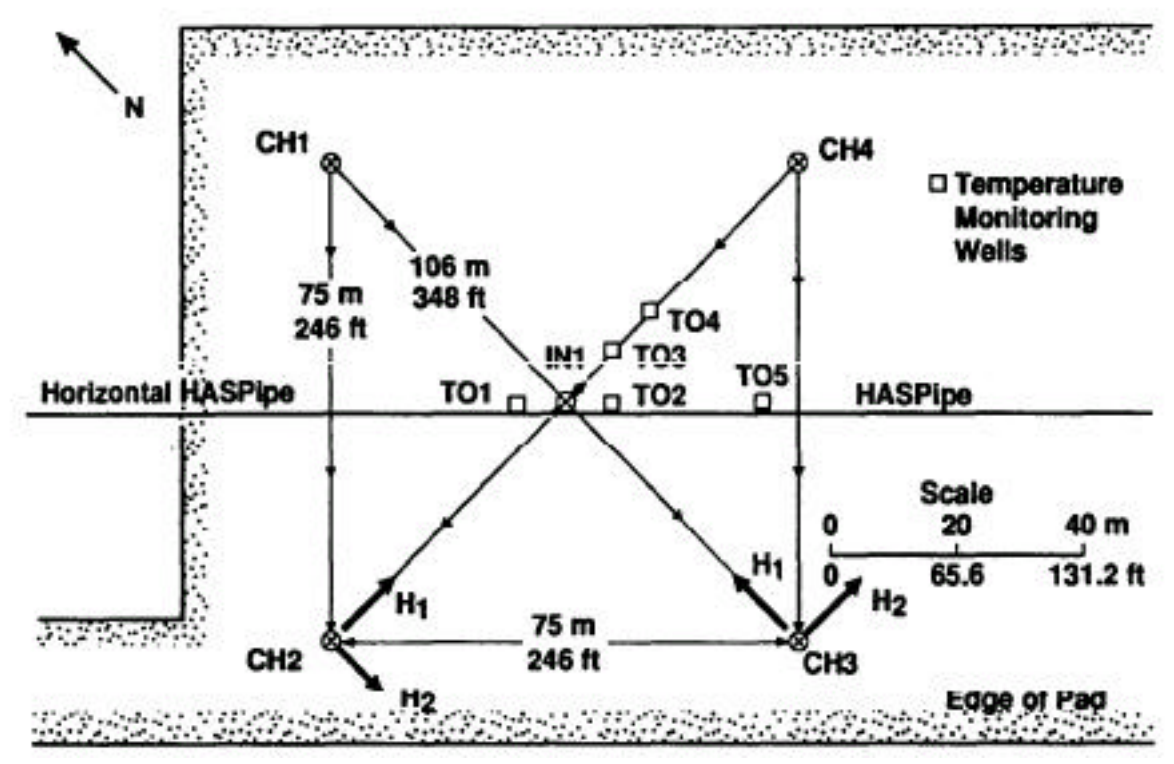

17. The crosswell seismic data interpreted comes from two experiments where

a. $\mathrm{CH} 1$ was source and $\mathrm{CH} 3$ receiver

b. $\mathrm{CH} 4$ was source and $\mathrm{CH} 2$ receiver

18. The objective of the entire crosswell seismic experiment (two surveys, one at 72 days after the other) was to define the geology and to monitor the movement of the steam injected between the surveys

19. There were two surveys. Because the second experiment interpreted the field after a period of steam injection, this project analyze only the $1^{\text {st }}$ experiment

20. For each of the lines (CH1-CH3 AND CH2- $\mathrm{CH} 4) 56$ seismic traces for the surface seismic and 56 seismic traces for the crosswell seismic were identified

21 . The trace spacing is 2 meters, and sample interval is 0.5 meters; they provide information from the surface- 0 meters to 320 meters

22. We looked for those traces that have both surface seismic and crosswell seismic amplitude. The surface seismic data cover all 56 traces

23. As a consequence of the crosswell survey's limited shot and geophone depth range, there are many traces (and portions of traces) with zero amplitude; they represent the area between the two wells that received no reflections.

24. We identified that the traces between the trace 16 and the trace 41 has, between 110-300 meters met the criteria- this is valid for both lines- 52 traces in total

25. There were 381 records available for each seismic attribute, for each trace 
26. We used 40 surface seismic traces for the training set, 8 surface seismic traces for the calibration set and 4 surface seismic traces for the verification set

27. They were 20 Artificial Neural Networks trained in order to prove that the methodology is robust

28. The method used in training is the General Regression Neural Network; the backpropagation neural networks gave, for this project, worse results, and the results are not listed here

29. The analysis suggest that all the networks trained gave good results (all the statistical indicators are positive- $\mathrm{R}^{2}, \mathrm{r}^{2}$, correlation coefficient)

30. The best network analyzed is network 12

31. For the best network:

o the training traces were the traces $16 \div 18,20 \div 23,25,27 \div 29,31 \div 33,35,36,38 \div 41$ for the wells $\mathrm{CH} 1-\mathrm{CH} 3$ and $\mathrm{CH} 2-\mathrm{CH} 4$

○ the calibration traces were the traces $24,26,34,37$ for the experiment including the wells $\mathrm{CH} 1-\mathrm{CH} 3$ and $\mathrm{CH} 2-\mathrm{CH} 4$

o the verification traces were the traces 19 and 30 for the experiments described above

○ the input and the output parameters were taken at the current depths. The analysis haven't shown an improvement when supplementary data from the surrounding zones were taken into account (data recorded 0.5 or one meters above or below)

○ the Appendix E gives the shape of the real crosswell seismic traces as well as the neural network prediction

○ the appendix F plot the real vs. the predicted crosswell seismic attributes

○ the statistical parameters for the best prediction (the network 12) gives, for the verification set the following values: 


\begin{tabular}{|l|c|c|c|}
\hline \multicolumn{1}{|c|}{ Seismic attribute } & $\mathrm{R}$ squared & $\mathrm{r}$ squared & Correlation coefficient \\
\hline \hline The acoustic impedance & 0.8113 & 0.8145 & 0.9025 \\
\hline The trace amplitude & 0.7509 & 0.7539 & 0.8683 \\
\hline The instantaneous phase & 0.5028 & 0.5061 & 0.7114 \\
\hline The instantaneous frequency & 0.7424 & 0.7433 & 0.8622 \\
\hline The trace envelope & 0.8631 & 0.8632 & 0.9291 \\
\hline
\end{tabular}

32. The prediction is very good for the acoustic impedance, trace amplitude, instantaneous frequency and the envelope

33. The seismic attribute most difficult to predict is the instantaneous phase. Even for this the statistical parameters are positive and the predicted traces follows reasonably the real crosswell seismic traces

34. For the acoustic impedance, the correlation between the predicted and the real values (verification set) is:

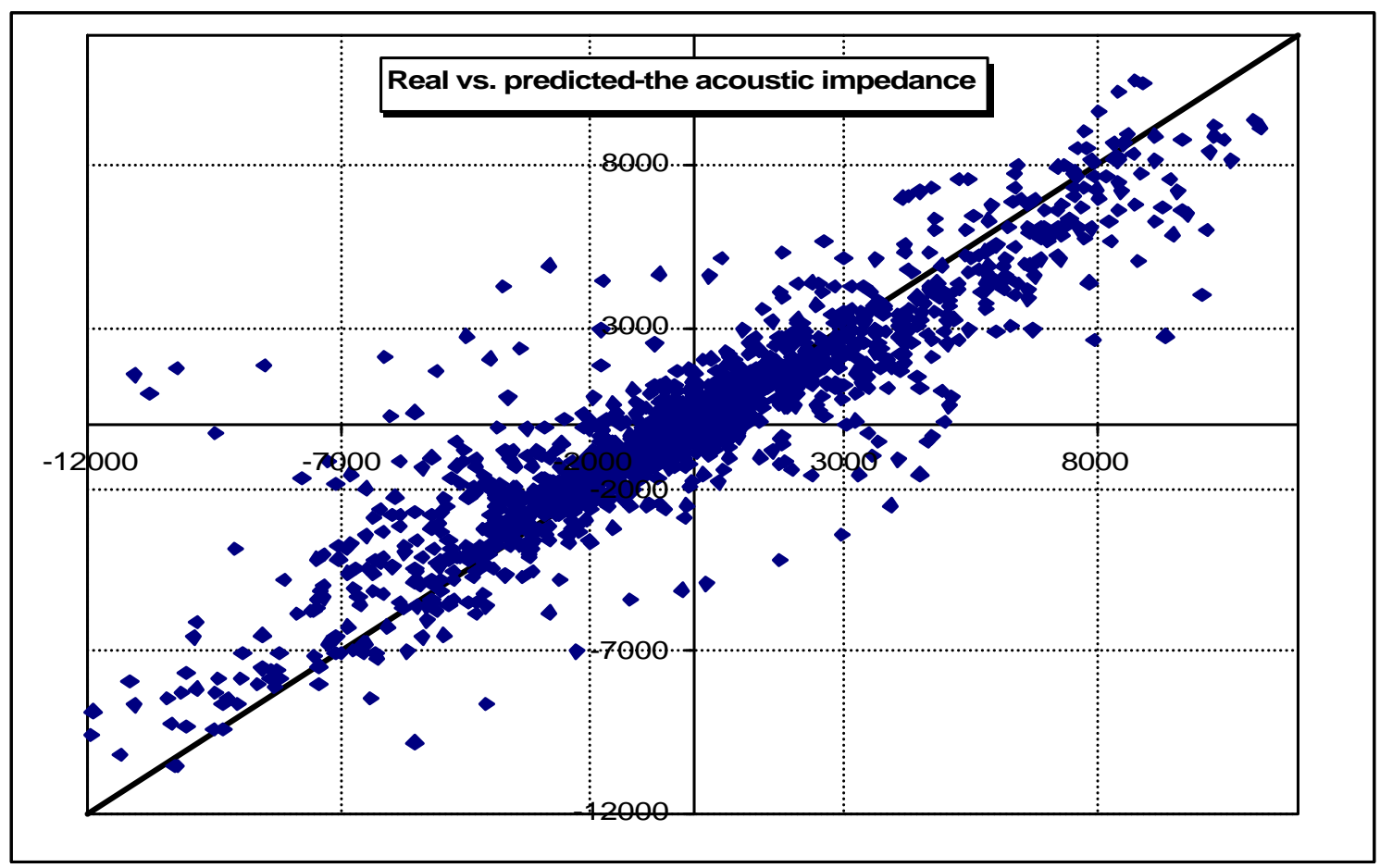


35. The crosswell seismic acoustic impedance for the trace 19 ( $\mathrm{CH} 2-\mathrm{CH} 4$ line), as resulted from the approach presented in this study is:

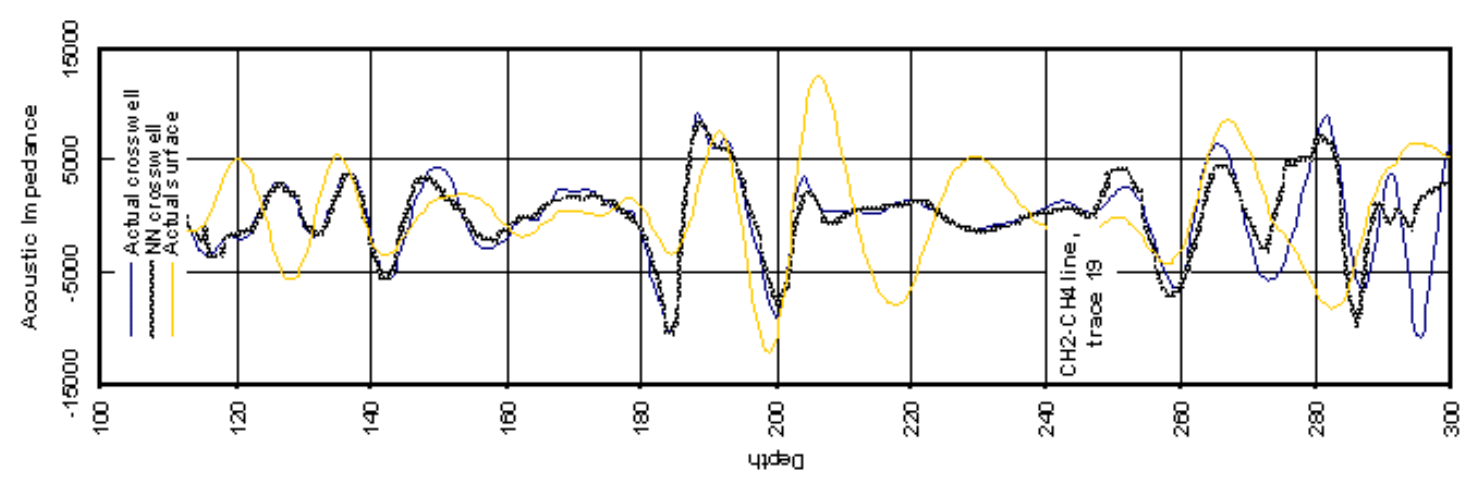

36. The mapping surface seismic- crosswell seismic can increase the chance of many petroleum industry experiments: EOR, IOR, drilling, etc.

37. The methodology presented here has a major impact in the petroleum industry. By our known it is the first time when this methodology is implemented. Further research could increase dramatically the resolution of the seismic methods at the field scale with a small increase in the cost of the experiments 


\section{REFERENCES}

1. Anstey, N.A. "Simple seismic" IHRDC Boston 1982

2. Badley, Michael E "Practical seismic interpretation” IHRDC Boston 1985

3. Balch, R.S. et al "Using artificial intelligence to correlate multiple seismic attributes to reservoir properties" SPE paper 56733

4. Berryman, J.G. "Nonlinear inversion and tomography"

http://sepwww.stanford.edu/sep/berryman/NOTES/lecture_notes.html

5. Bhuiyan, Moffazal: An Intelligent System's Approach To Reservoir Characterization In Cotton Valley, MS Thesis, West Virginia University, 2001

6. Brown , A. R. "Interpreter's corner - Seismic attributes and their classification” The Leading Edge October 1996 Vol. 15, No. 10

7. Chawathe, A et al "Interwell property mapping using crosswell seismic attributes" SPE paper 38747

8. Chemometrics Research Group "General Regression Neural Networks" http://chemdiv-www.nrl.navy.mil/6110/sensors/chemometrics/grnn.html

9. Chen, C.H. "Fuzzy logic and neural network handbook" McGraw- Hill

10. Clark, D.W. "An introduction to neural networks"

http://members.home.net/neuralnet/introtonn/index.htm

11. Cooke, D et al "What is the best seismic attribute for quantitative seismic reservoir characterization? "1999 SEG Expanded Abstracts

http://www.seg.org/meetings/past/seg1999/techprog/detail/SRC1.html 
12. Cooper, N.M. "The Value of 3D Seismic"

http://www.anwr.org/techno/3dseismi.html

13. Fairborn, John (WellSeismic Computing Services) Reflection imaging-Steepbank- surface and crosswell data, final report, July 2001

14. Fairborn, John (WellSeismic Computing Services) A note about the resolution of the seismic experiments- Steepbank field, July 2001

15. Fournier, F and Derain, JF "A statistical methodology for deriving reservoir properties from seismic data" Geophysics Vol. 60, No. 5 September- October 1995

16. Gadallah, Mamdouh R. "Reservoir seismology"

17. Gourrion, J. "General regression neural networks"

http://www.ifremer.fr/droos/Perso/gourrion/rapport/node14.html

18. Harris, Jerry $\mathrm{M}$ et al "High-resolution crosswell imaging of a West Texas carbonate reservoir: Part 2: Wavefield modeling and analysis" Geophysics Vol. 60, No. 3 May-June 1995

19. Hart, D. et al "Time to depth conversion of the Nash Draw ' $L$ ' seismic horizon using seismic attributes and neural networks" SPE Paper 59555

20. Jackson, M.J. and Tweeton, D.R. "Program MIGRATOM for Crosswell Radar and Seismic Tomography" U.S. Geological Survey Open-File Report 00-0457 Version 1.0 available at http://greenwood.cr.usgs.gov/pub/open-file-reports/ofr-00-0457/ 
21. Jackson, M.J. and Tweeton, D.R. "Program 3DTOM for Crosswell Radar and Seismic Tomography" U.S. Geological Survey Open-File Report 00-0456 Version 1.0 available at http:/greenwood.cr.usgs.gov/pub/open-file-reports/ofr-00-0456/

22. Kosko, B “Neural Networks and Fuzzy Systems”, Prentice-Hall, 1991

23. Latimer, R.B. et al "An interpreter's guide to understanding and working with seismic-derived acoustic impedance data" The Leading Edge March 2000 Vol. 19, No. 3

24. Lazaratos, Spyros M et al "High-resolution crosswell imaging of a West Texas carbonate reservoir: Part 4: Reflection imaging" Geophysics Vol. 60, No. 3 May-June 1995

25. Lee, D.S. et al "High-resolution crosswell seismic experiment with a large interwell spacing in a West Texas carbonate field" Geophysics May-June 1995 Vol. 60, No. 3

26. Liner, C and Bozkurt, G "Geophysical Aspects of the Glennpool Project" http://www.mcs.utulsa.edu/ cll/academic/DoeRpt.html

27. Lines, Larry R. et al "Integrated interpretation of borehole and crosswell data from a west Texas field" The Leading Edge January 1993 Volume 12, Number 1

28. Lines, Larry et al "Integrated reservoir characterization: Beyond tomography" Geophysics Vol. 60, No. 2 March-April 1995

29. Link, C.A. et al "Crosshole tomography in the Seventy-six West Field" The Leading Edge, January 1993 
30. Macrides, C.G. et al "Reservoir Methods and case studies"

http://www.cseg.org/events/meetings/abstracts/1999/rmcs_ch5.pdf

31 Marsden , D. "Static corrections-a review, part 2" The Leading Edge, January- March 1993

32. McVey, D.S. et al "Identification of Parameters Influencing the Response of Gas Storage Wells to Hydraulic Fracturing With the Aid of a Neural Network" SPE paper 29159. Published in SPE Computer Applications Journal, April 1996

33. Mohaghegh, S "Virtual Intelligence Applications in Petroleum Engineering: Part 1 - Artificial Neural Networks"- JPT September 2000 - Distinguished Author Series.

34. Mohaghegh, S. "Virtual Intelligence Applications in Petroleum Engineering: Part 2 Evolutionary computing"- JPT September 2000 - Distinguished Author Series.

35. Mohaghegh, S. "Virtual Intelligence Applications in Petroleum Engineering: Part 3 - Fuzzy logic"- JPT September 2000 - Distinguished Author Series.

36. Mohaghegh, S. et al "Design and Development of An Artificial Neural Network for Estimation of Formation Permeability" SPE paper 28237, SPE Computer Applications, December 1995

37. Mohaghegh, S. et al "State-of-the-Art in Permeability Determination From Well Log Data; Part 1: A Comparative Study, Model Development." SPE paper 30978. Published in SPE Formation Evaluation Journal, September 1997.

38. Mohaghegh, S. et al "State-of-the-Art in Permeability Determination From Well Log Data; Part 2: Verifiable, Accurate Permeability Predictions, the Touch Stone of All Models." SPE paper 30979. Published in SPE Formation Evaluation Journal, September 1997.

39. Mohaghegh, S “An Intelligent Systems Approach for High Resolution, Interwell Oil Reservoir Characterization" WVU report, 2000 
40. Mufti , Irshad R. "Pitfalls in crosshole seismic interpretation as a result of 3-D effects" Geophysics Vol. 60, No. 3 May-June 1995

41. Naval Facilities Engineering Service Center "High resolution 3-d seismic reflection survey" http://enviro.nfesc.navy.mil/erb/erb_a/restoration/technologies/invest/geo_phys/3d/srsatt1.htm

42. Paulsson, B.N.P. et a "The Steepbank crosswell seismic project: reservoir definition and evaluation of steamflood technology in Alberta tar sands" The Leading Edge, July 1994

43. M.C. de Roos "Neural network as an alternative to rock physics modeling in time lapse seismic reservoir monitoring" OTC 13162, JPT June 2001

44. Neep, J.P "Measurement of seismic attenuation from high-resolution crosshole data" Geophysics Vol. 61, No. 4 July-August 1996

45. Rector, James W et al "High-resolution crosswell imaging of a West Texas carbonate reservoir: Part 3: Wavefield separation of reflections" Geophysics Vol. 60, No. 3 May-June 1995

46. Ronek, S et al "Seismic-guided estimation of log properties, part 2: Using artificial neural networks for nonlinear attribute calibration” The Leading Edge, June 1994

47. Schultz , P.S. et al "Seismic-guided estimation of log properties, part 1: A data-driven interpretation methodology" The Leading Edge, May 1994

48. Schultz , P.S. et al "Seismic-guided estimation of log properties, part 3: A controlled study" The Leading Edge, July 1994

49. Schuster, G "Basics of Traveltime Tomography" available online at http://utam.geophys.utah.edu/stanford/node25.html 
50. Soto , R.B. and Holditch, S.A. "Development of reservoir characterization models using core, well log, and 3d seismic data and intelligent software” SPE paper 57457

51. Specht, Donald F. “A General Regression Neural Network”- IEEE Transactions Of Neural Networks Vol 2, No. 6, November 1991

52. Stergiou, C and Siganos, D "Neural networks"

http://www.doc.ic.ac.uk/ nd/surprise_96/journal/vol4/cs11/report.html

53. Takahashi, et al "A strategy to select optimal seismic attributes for reservoir property estimation: Application of information theory" 1999 SEG Expanded Abstracts http://www.seg.org/meetings/past/seg1999/techprog/detail/SRC1.html

\section{Taner, Turhan M “ATTRIBUTES REVISITED”}

http://www.rocksolidimages.com/pdf/attrib_revisited.htm

55. Trappe, $\mathrm{H}$ and Hellmich, $\mathrm{C}$ "Using neural networks to predict porosity thickness form 3D seismic" The first break September 2000

56. Tweeton, D.R. "Program BOMTOM for Crosswell Radar and Seismic Tomography" U.S. Geological Survey Open-File Report 00-0455 Version 1.0 available at http://greenwood.cr.usgs.gov/pub/open-file-reports/ofr-00-0455/

57. Tweeton, D.R. Geotom3D program- personal communication

58. Tycker, K.E. et al "Geologic investigation of cross-well seismic response in a carbonate reservoir, McElroy field, West Texas” AAPG Bulletin, V 82, No. 8 August 1998

59. Zhow, $\mathrm{C}$ et al "High-resolution cross-well imaging by seismic traveltime + waveform inversion” The Leading Edge October 1993, Vol. 12, No. 10 
60. Walls , J et al "North Sea reservoir characterization using rock physics, seismic attributes, and neural networks: A case history “ 1999 SEG Expanded Abstracts http://www.seg.org/meetings/past/seg1999/techprog/detail/SRC1.html

61. Wei Liu, Gordon F. West "Time-lapse crosswell seismic imaging of the Athabasca tar sands at Steepbank" 1998 SEG Expanded Abstracts

62. White, A.C. , Mohaghegh, S. et al "The Application of Artificial Neural Networks to Zone Identification in a Complex Reservoir" SPE paper 30977

63. Williamson, P.R. "Crosshole imaging in anisotropic media" The Leading Edge January 1993, Vol. 12, No. 1

64. "Neural Network Computing"

http://sunflower.singnet.com.sg/ midaz/Intronn.htm

65. "Seismic attributes"

http://www.beg.utexas.edu/agi/mod04/m04_hil01.htm

66. "What are Artificial Neural Networks?"

http://www.accurate-automation.com/products/nnets.htm

67. "What is GRNN?"

http://www.faqs.org/faqs/ai-faq/neural-nets/part2/section-20.html 


\section{APPENDIX A- The trace attributes plot}

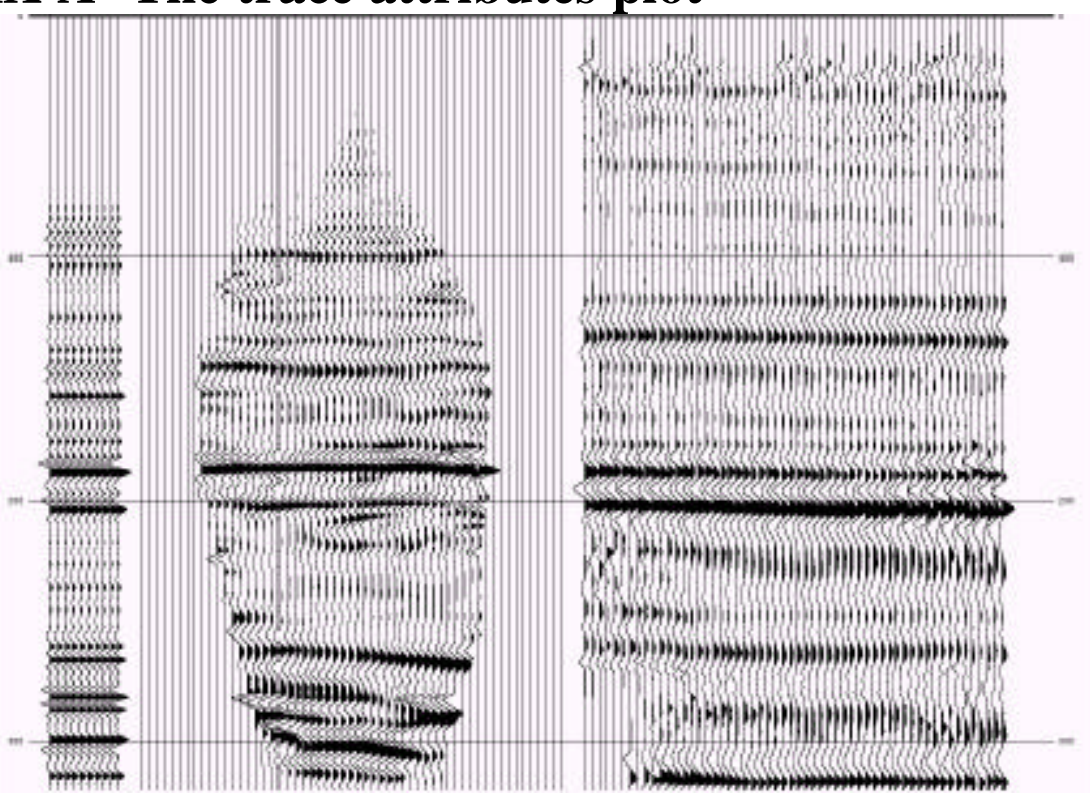

Figure A-1: Ch2-Ch4 trace amplitudes

Panel 1: 10 identical synthetic traces

Panel 2: Reflections from crosswell survey

Panel 3: Reflections from surface survey

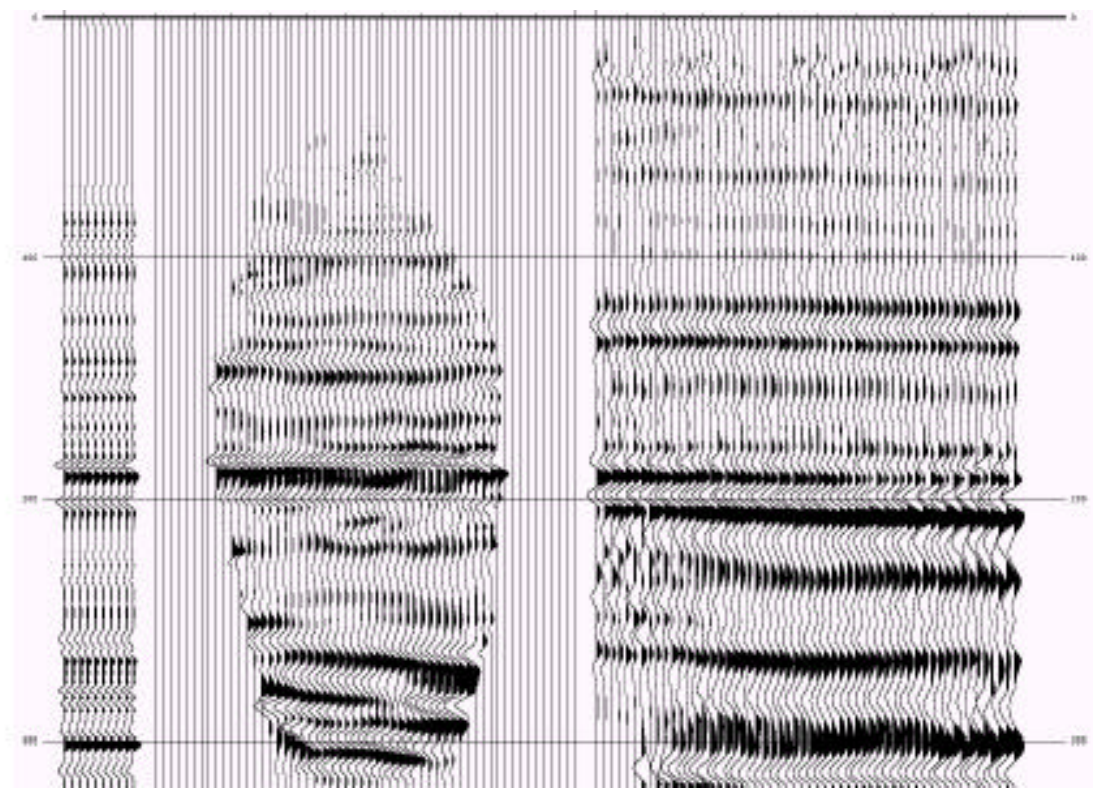

Figure A-2: Ch2-Ch4 acoustic impedance

Panel 1: 10 identical synthetic traces

Panel 2: Reflections from crosswell survey

Panel 3: Reflections from surface survey 


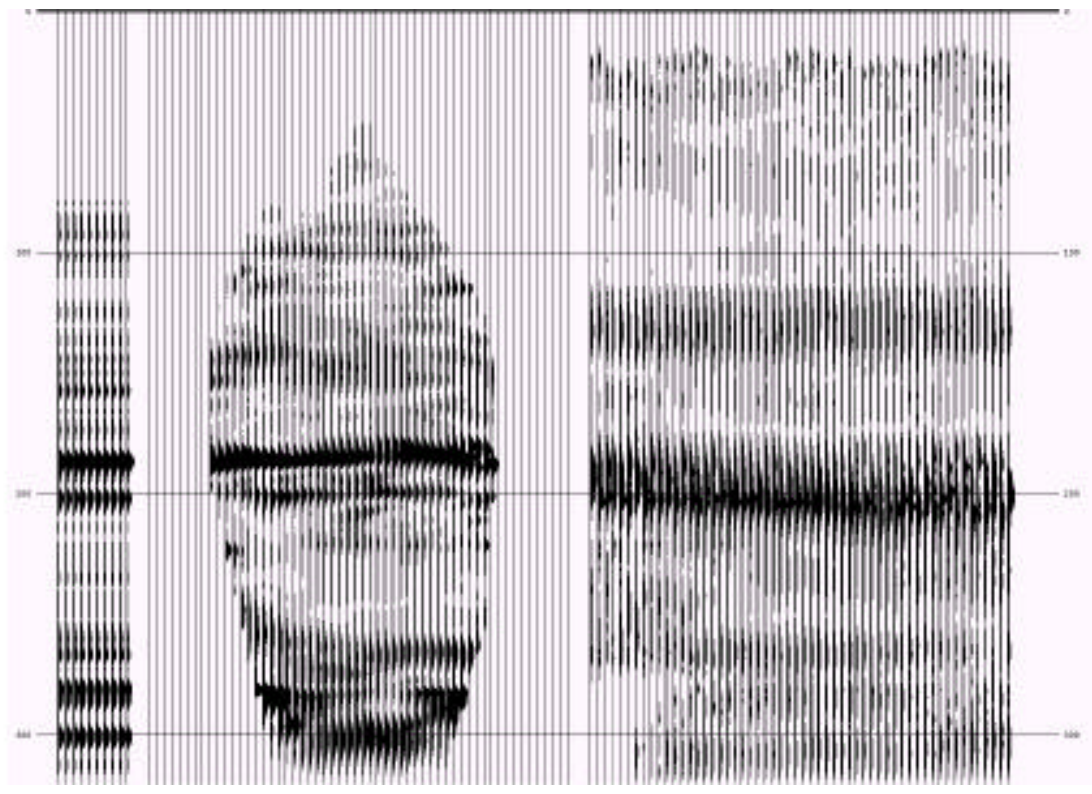

Figure A-3: $\mathrm{Ch} 2-\mathrm{Ch} 4$ trace envelope

Panel 1: 10 identical synthetic traces

Panel 2: Reflections from crosswell survey

Panel 3: Reflections from surface survey

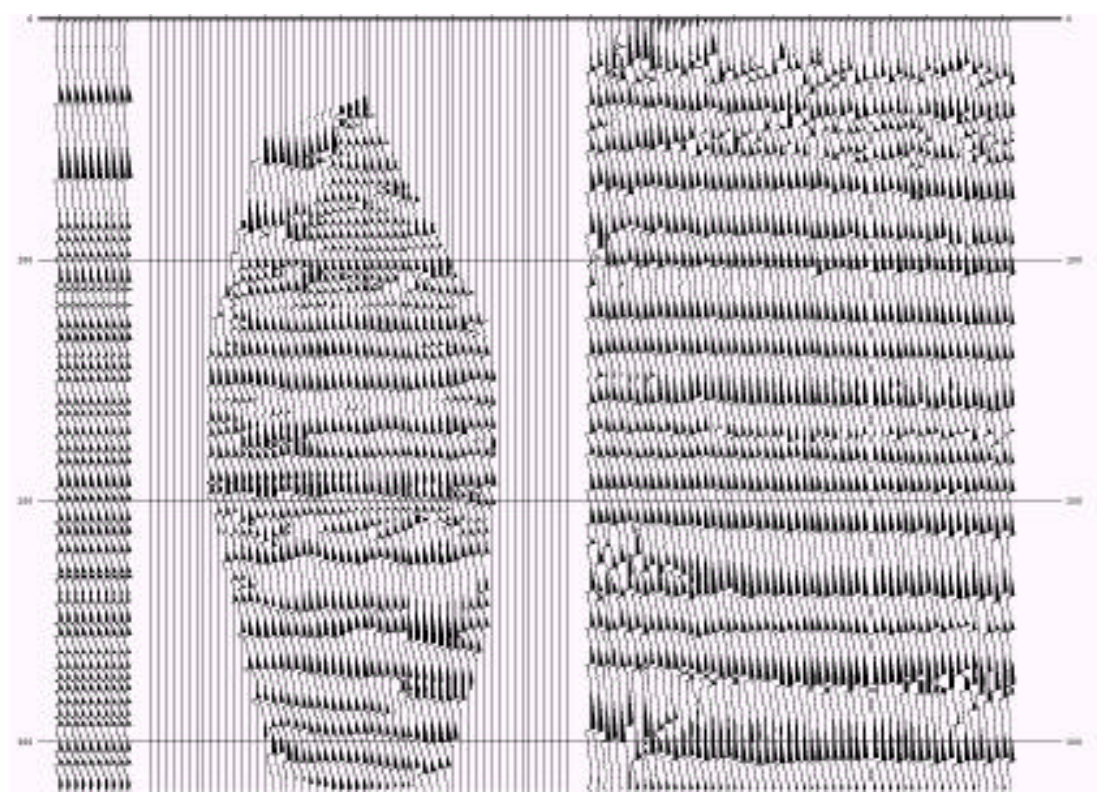

Figure A-4: Ch2-Ch4 Instantaneous phase

Panel 1: 10 identical synthetic traces

Panel 2: Reflections from crosswell survey

Panel 3: Reflections from surface survey 


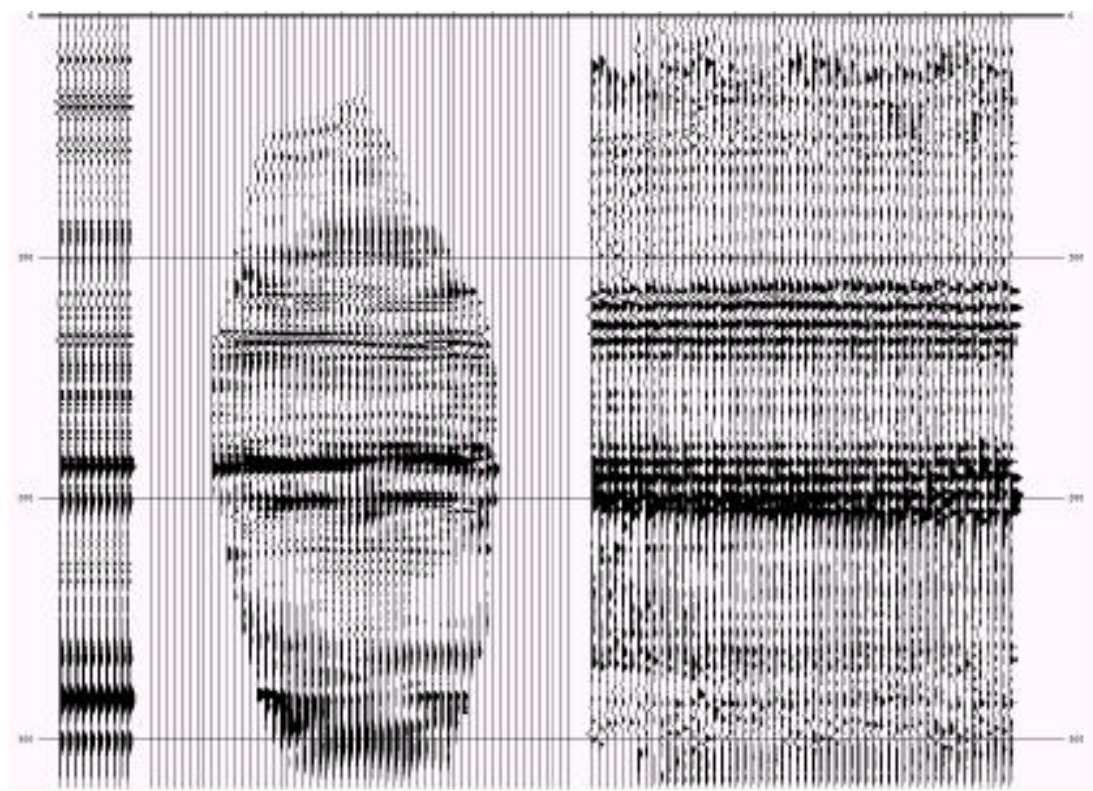

Figure A-5: Ch2-Ch4 Instantaneous frequency

Panel 1: 10 identical synthetic traces

Panel 2: Reflections from crosswell survey

Panel 3: Reflections from surface survey

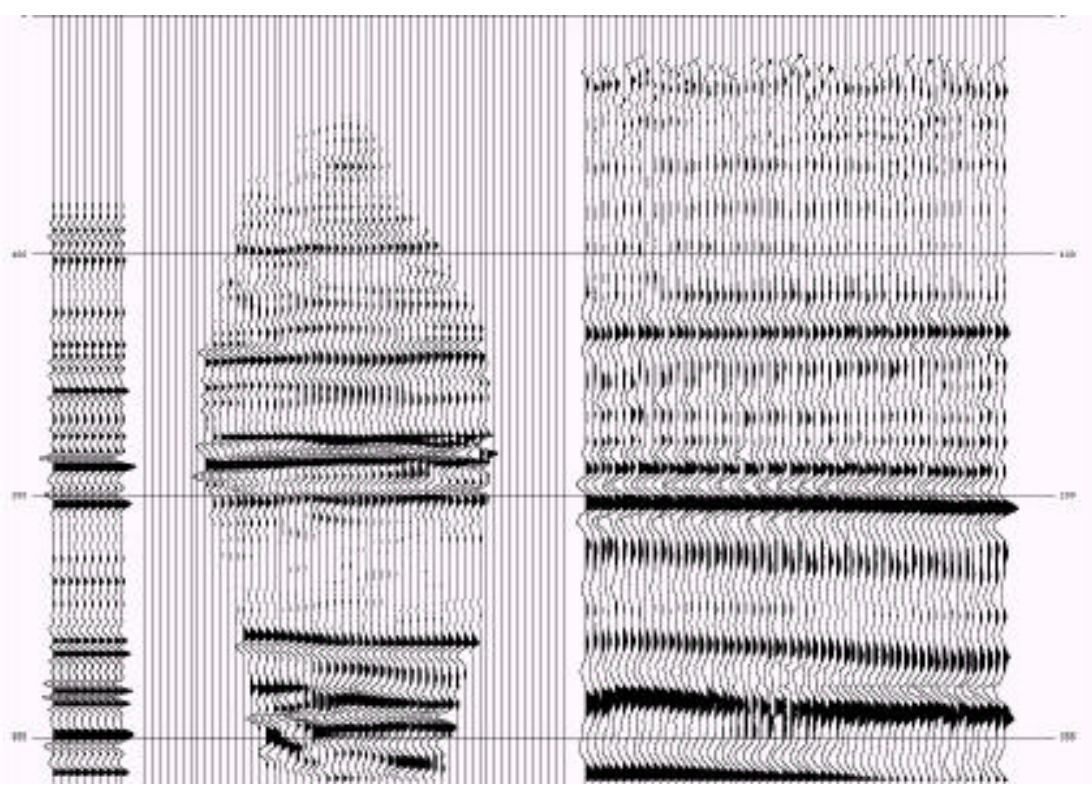

FigureA- 6: Ch1-Ch3 Trace amplitudes

Panel 1: 10 identical synthetic traces

Panel 2: Reflections from crosswell survey

Panel 3: Reflections from surface survey 


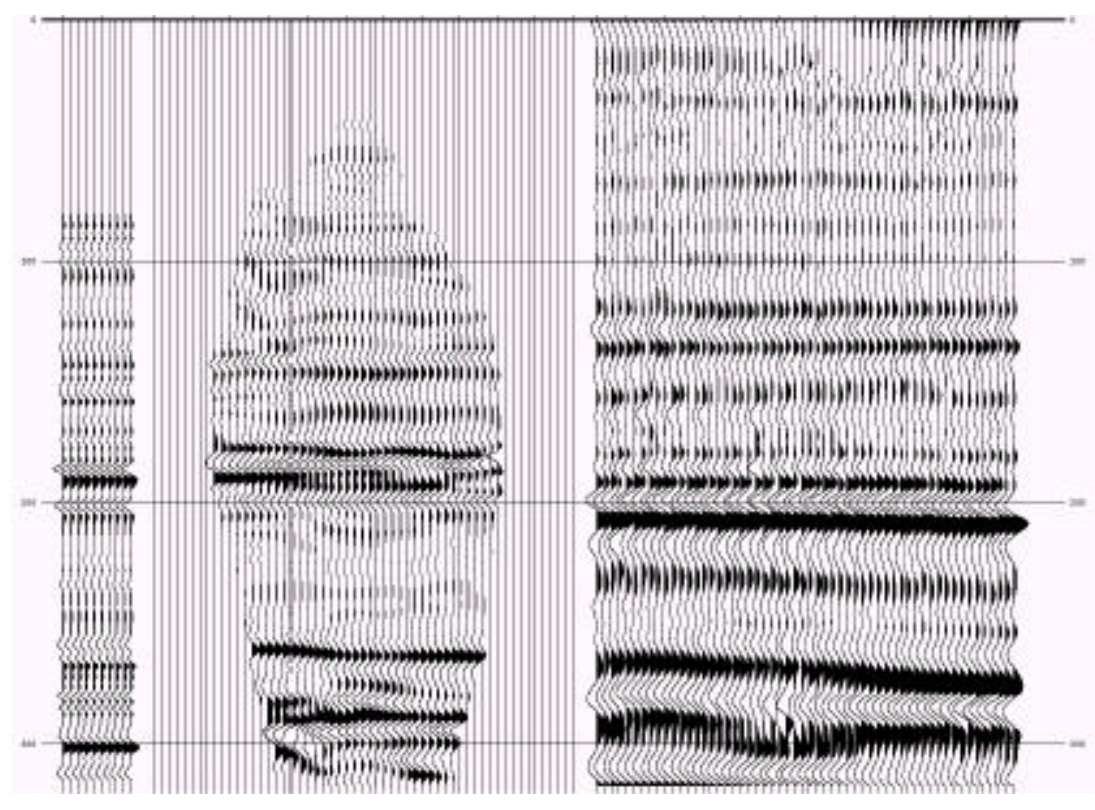

Figure A-7: Ch1-Ch3 Acoustic impedance

Panel 1: 10 identical synthetic traces

Panel 2: Reflections from crosswell survey

Panel 3: Reflections from surface survey

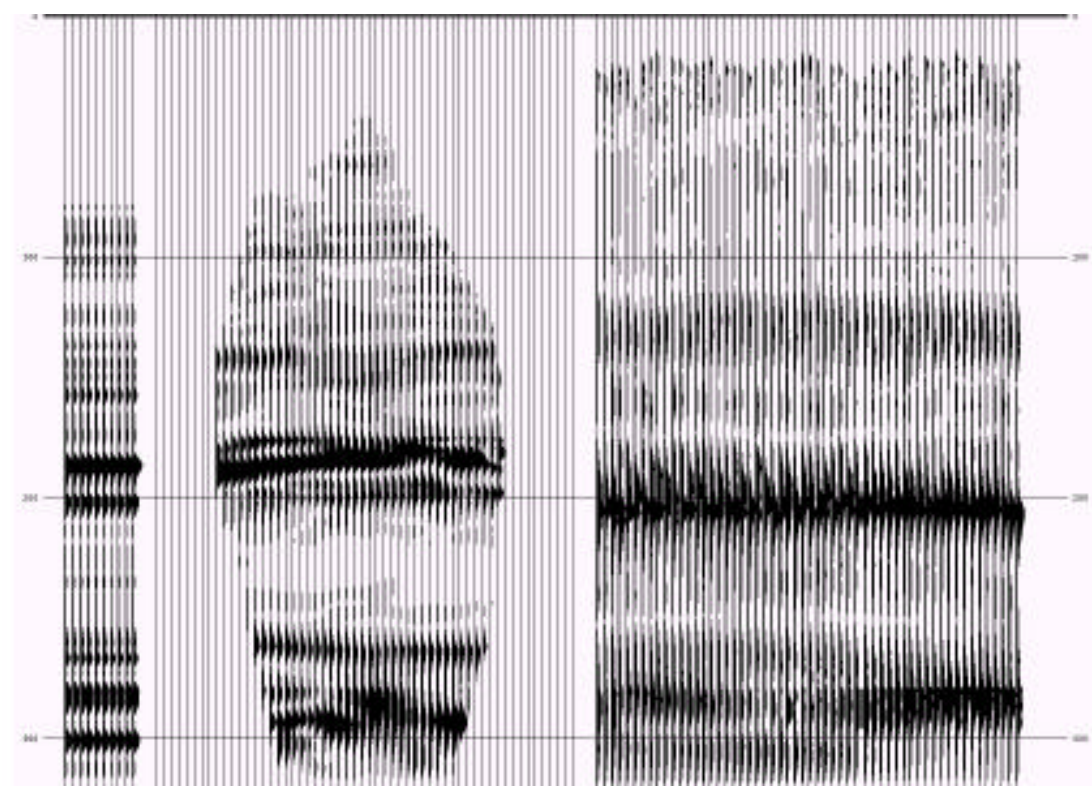

Figure A-8: Ch1-Ch3 Trace envelope

Panel 1: 10 identical synthetic traces

Panel 2: Reflections from crosswell survey

Panel 3: Reflections from surface survey 


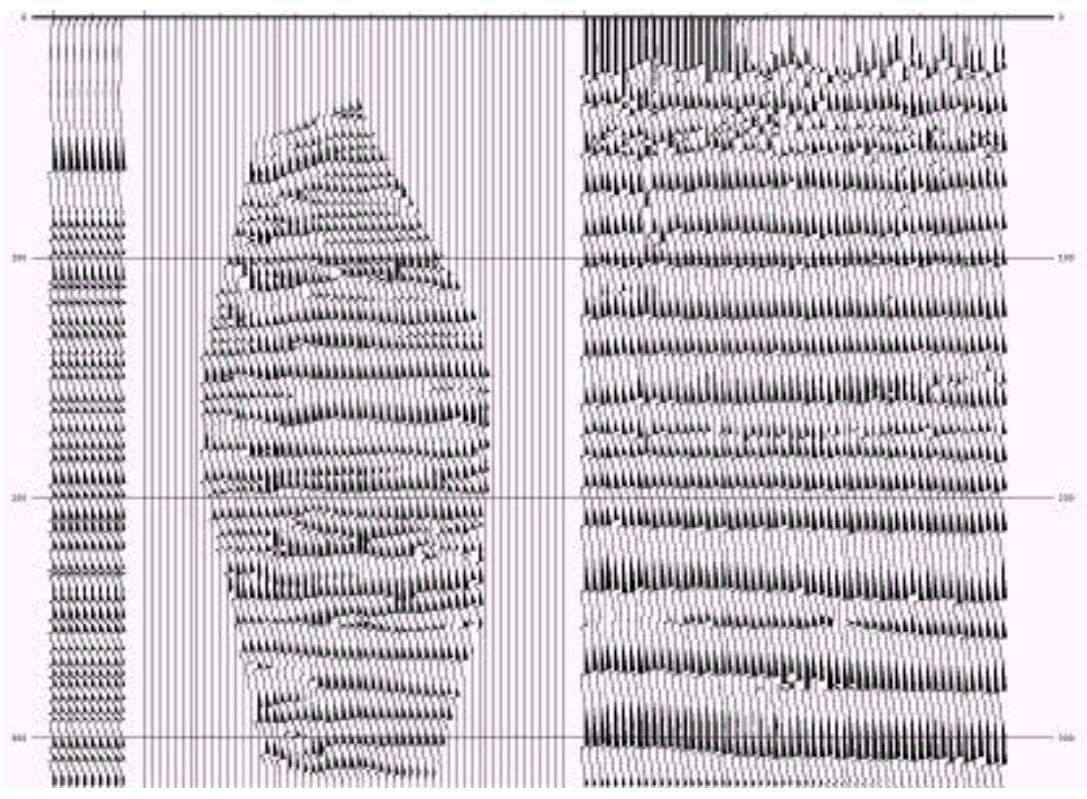

Figure A-9: Ch1-Ch3 Instantaneous phase

Panel 1: 10 identical synthetic traces

Panel 2: Reflections from crosswell survey

Panel 3: Reflections from surface survey

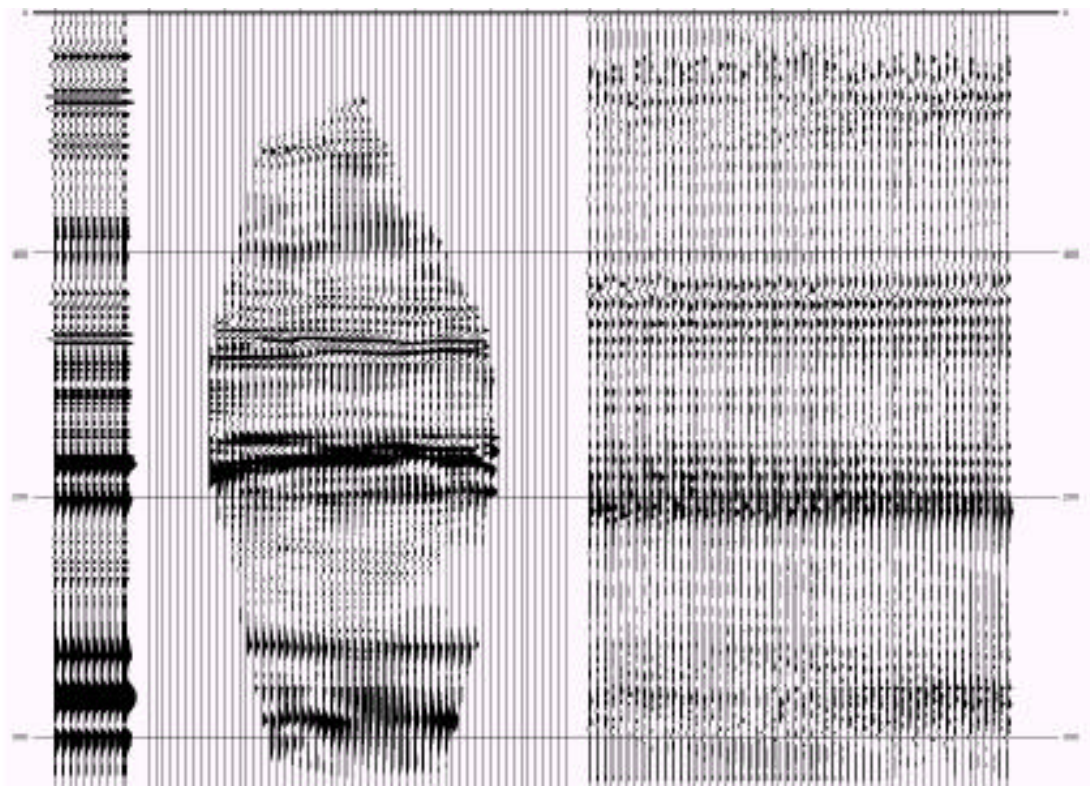

Figure A-10: Ch1-Ch3 Instantaneous frequency

Panel 1: 10 identical synthetic traces

Panel 2: Reflections from crosswell survey

Panel 3: Reflections from surface survey 


\section{APPENDIX B The data spectra for the surface seismic and for the crosswell seismic for $\mathrm{CH1}-\mathrm{CH}$. These spectra are also representative of the $\mathrm{CH} 2-\mathrm{CH} 4$ sections.}
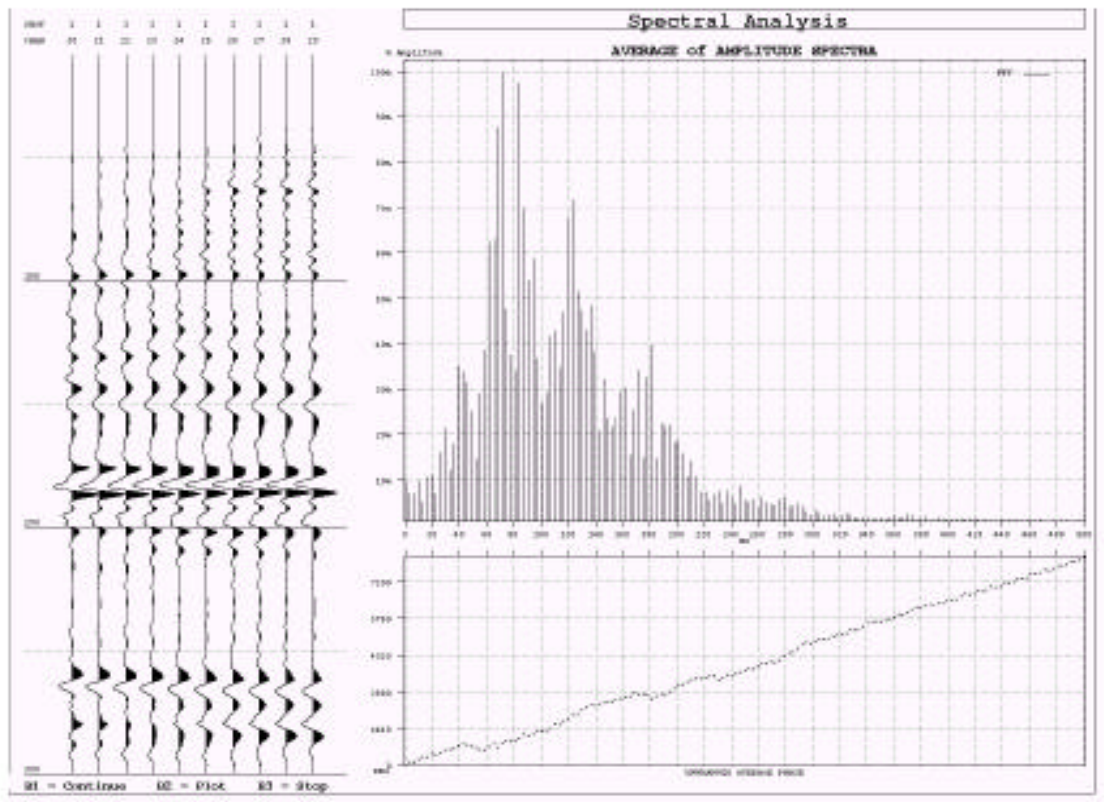

Figure B1. Data spectra for the crosswell seismic.
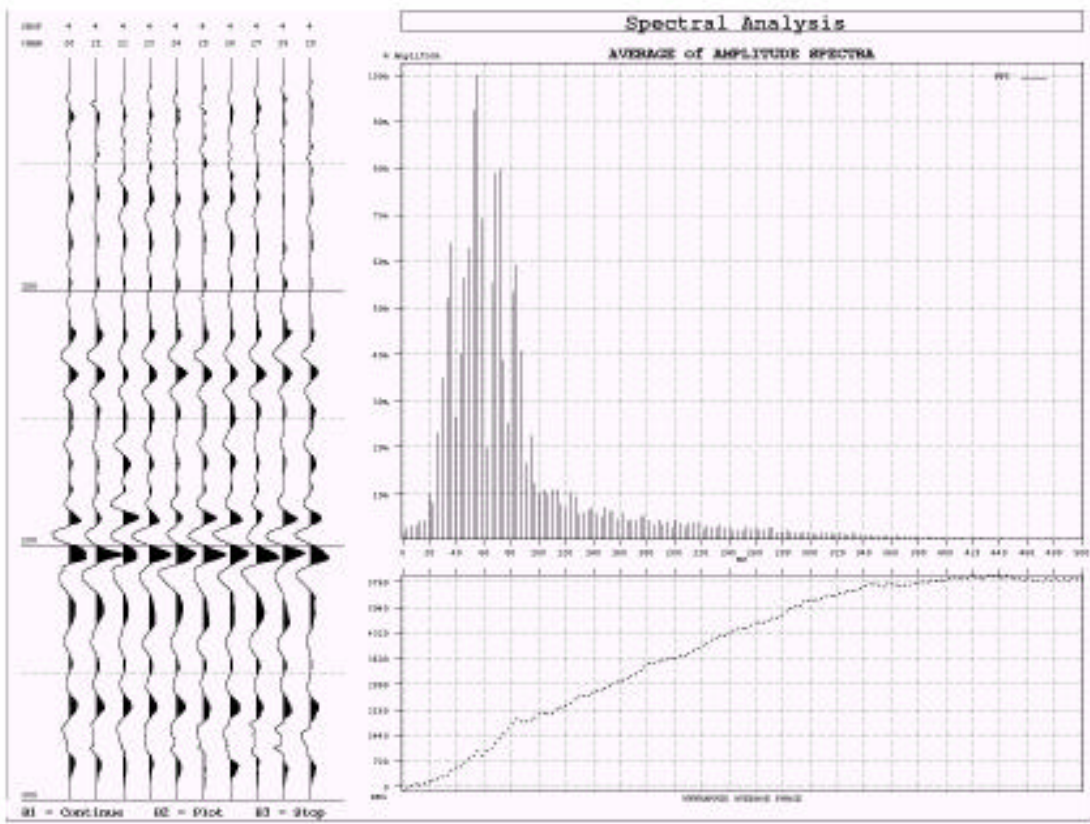

Figure B2. Data spectra for the surface seismic. 


\section{Appendix C - COMPARISON BETWEEN THE REAL TRACE SHAPE IN THE SURFACE SEISMIC AND THE CROSSWELL SEISMIC TRACE - trace 19, CH2- CH4 line}
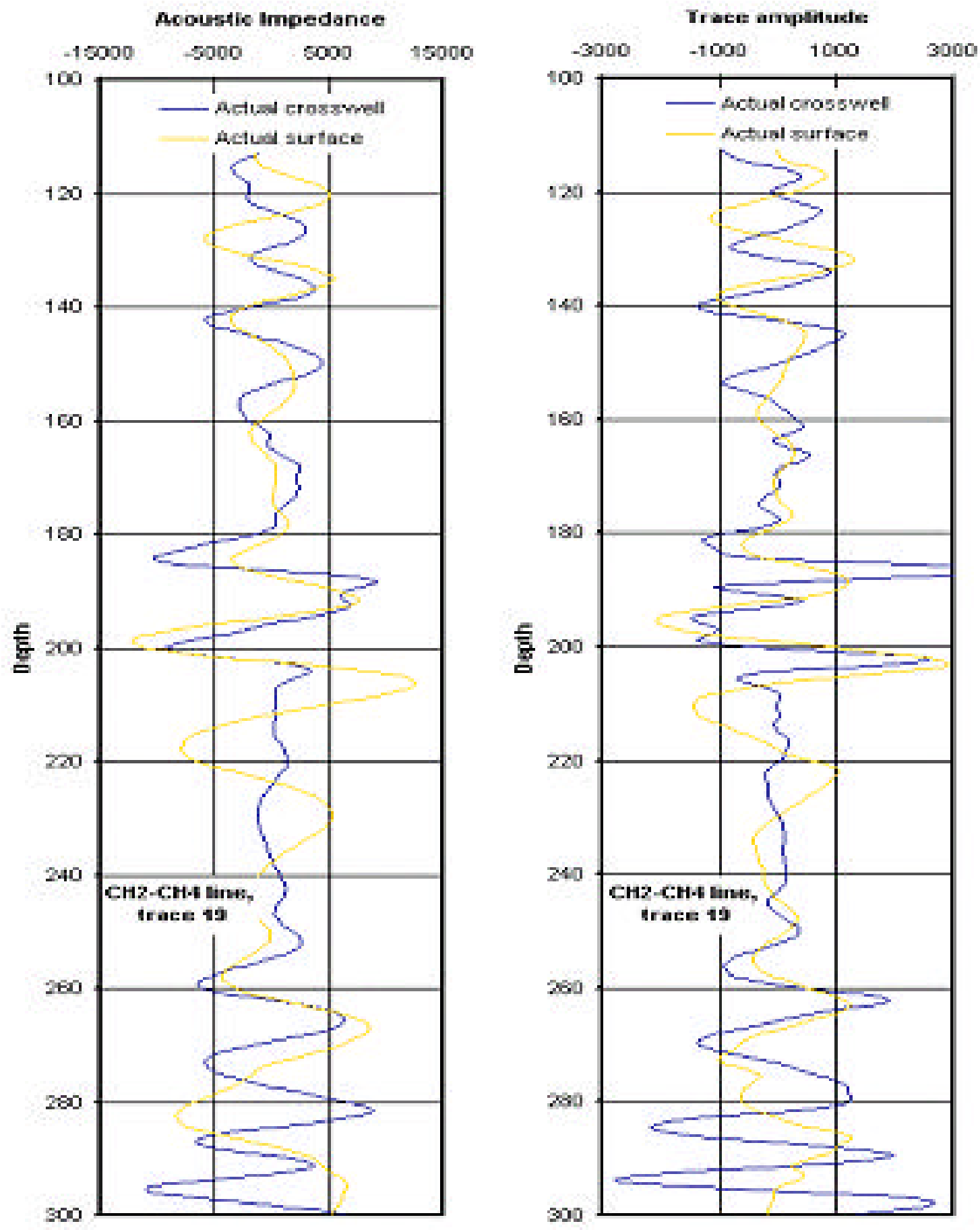

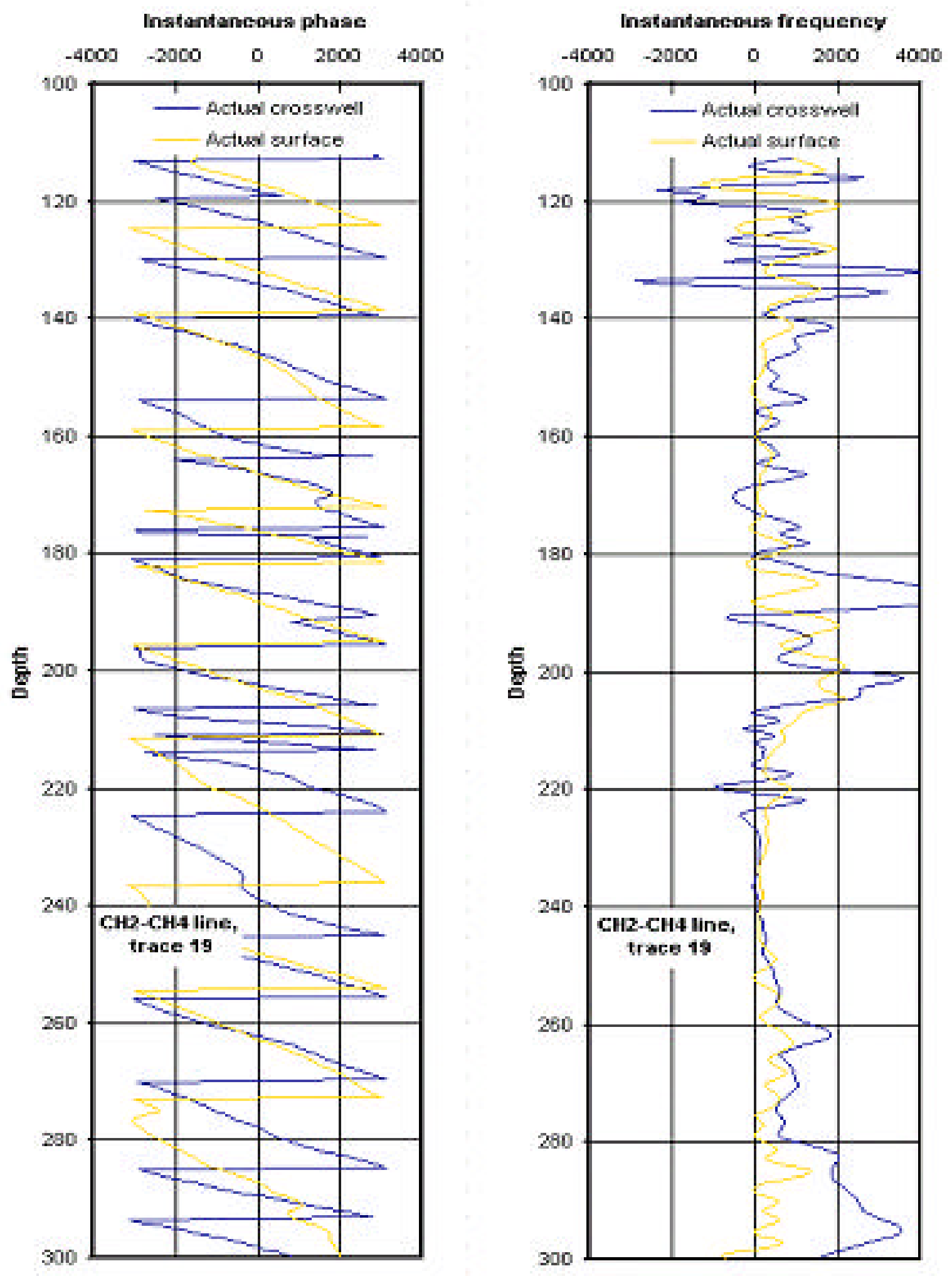


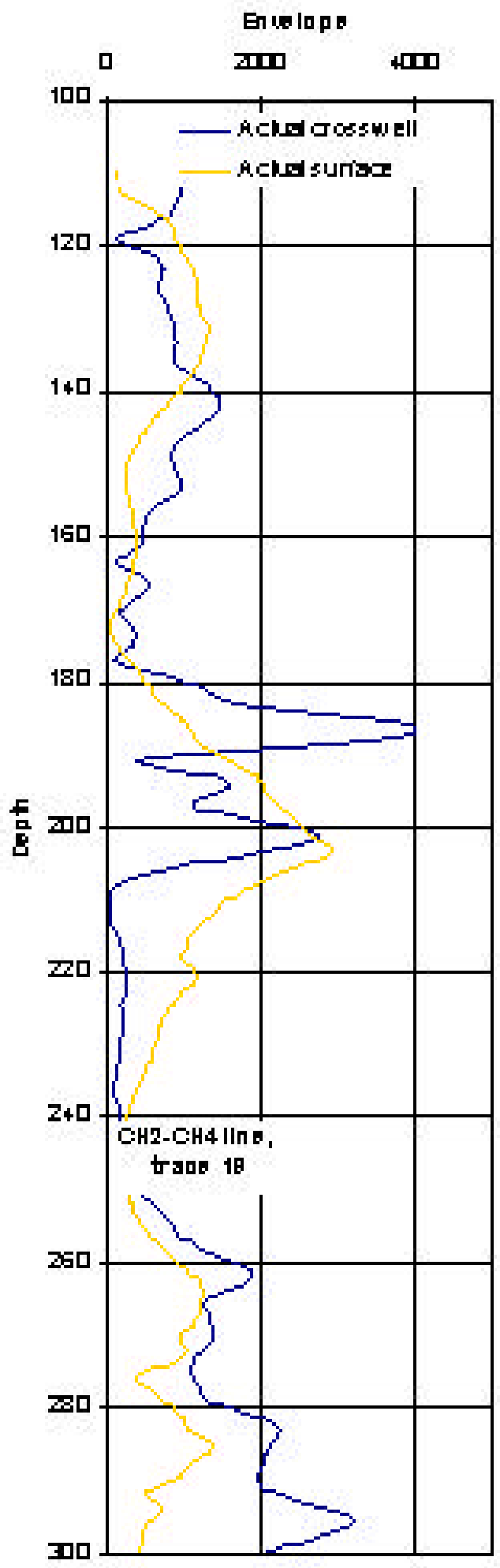


Appendix D- Statistics on prediction goodness for training, calibration and verification datasets.

\begin{tabular}{|c|c|c|c|c|c|c|c|c|c|c|c|c|c|c|c|}
\hline \multirow{3}{*}{ Network } & \multicolumn{15}{|c|}{ Training } \\
\hline & \multicolumn{5}{|c|}{ R squared } & \multicolumn{5}{|c|}{ r squared } & \multicolumn{5}{|c|}{ Correlation coefficient } \\
\hline & & & & & & & & & & & & & & $\mathrm{CA}$ & \\
\hline 1 & 0.8196 & $\frac{\mathrm{C}}{0.7926}$ & $\frac{\mathrm{L}}{0.56}$ & 0.7464 & $\frac{\mathrm{\omega}}{0.8802}$ & 0.8255 & $\frac{\mathrm{L}}{0.803}$ & 0.5708 & $\frac{C 4}{0.7534}$ & 0.8819 & 0.9086 & $\frac{\mathrm{C}}{0.8961}$ & 0.7555 & $\begin{array}{c}\mathrm{CA} \\
0.868\end{array}$ & $\frac{\text { C3 }}{0.9391}$ \\
\hline 2 & 0.7392 & 0.6966 & 0.4691 & 0.6723 & 0.8382 & 0.7687 & 0.7301 & 0.5015 & 0.69 & 0.8458 & 0.8768 & 0.8545 & 0.7082 & 0.8307 & 0.9197 \\
\hline 3 & 0.7753 & 0.7496 & 0.5272 & 0.7274 & 0.8664 & 0.8018 & 0.7777 & 0.5603 & 0.744 & 0.8739 & 0.8955 & 0.8819 & 0.7485 & 0.8626 & 0.9348 \\
\hline 4 & 0.7989 & 0.7859 & 0.5526 & 0.758 & 0.8903 & 0.8151 & 0.8011 & 0.5725 & 0.7683 & 0.8932 & 0.9029 & 0.8951 & 0.7566 & 0.8766 & 0.9451 \\
\hline 5 & 0.7762 & 0.7666 & 0.5292 & 0.7507 & 0.886 & 0.795 & 0.785 & 0.5542 & 0.7625 & 0.8897 & 0.8916 & 0.886 & 0.7444 & 0.8732 & 0.9432 \\
\hline 6 & 0.7492 & 0.7252 & 0.489 & 0.7158 & 0.8698 & 0.7735 & 0.7512 & 0.519 & 0.731 & 0.875 & 0.8795 & 0.8667 & 0.7204 & 0.8555 & 0.9354 \\
\hline 7 & 0.8354 & 0.8084 & 0.5912 & 0.7856 & 0.9123 & 0.8534 & 0.8284 & 0.624 & 0.8002 & 0.9167 & 0.9238 & 0.9102 & 0.7899 & 0.8945 & 0.9575 \\
\hline 8 & 0.7981 & 0.7657 & 0.5508 & 0.7634 & 0.9021 & 0.8217 & 0.7931 & 0.5913 & 0.7806 & 0.9081 & 0.9065 & 0.8906 & 0.769 & 0.8835 & 0.953 \\
\hline 9 & 0.8374 & 0.814 & 0.6177 & 0.8123 & 0.9317 & 0.8531 & 0.8338 & 0.6476 & 0.8265 & 0.9354 & 0.9237 & 0.9131 & 0.8048 & 0.9091 & 0.9672 \\
\hline 10 & 0.9134 & 0.8918 & 0.7092 & 0.8667 & 0.9672 & 0.9239 & 0.9084 & 0.734 & 0.8793 & 0.9692 & 0.9612 & 0.9531 & 0.8568 & 0.9377 & 0.9845 \\
\hline 11 & 0.8229 & 0.8035 & 0.5929 & 0.7967 & 0.942 & 0.8442 & 0.8332 & 0.6355 & 0.8122 & 0.9457 & 0.9188 & 0.9128 & 0.7972 & 0.9012 & 0.9725 \\
\hline 12 & 0.9162 & 0.8954 & 0.7164 & 0.8779 & 0.9601 & 0.9236 & 0.9053 & 0.7335 & 0.8858 & 0.9613 & 0.9611 & 0.9515 & 0.8564 & 0.9412 & 0.9805 \\
\hline 13 & & & & & & & & & & & & 25 & & 76 & 0.9792 \\
\hline 14 & 0.9511 & 0.9367 & 0.7985 & 0.9238 & 0.9747 & 0.9569 & 0.9446 & 0.8148 & 0.931 & 0.9761 & 0.9782 & 0.9719 & 0.9027 & 0.9649 & 0.988 \\
\hline 15 & 0.9518 & 0.9382 & & 0.9239 & 0.9751 & & 0.9458 & 0.8146 & 0.9305 & & 0.9784 & 0.9725 & & & 0.9881 \\
\hline 16 & 0.9434 & 0.9355 & 0.7875 & 0.9174 & 0.9712 & 0.9533 & 0.9448 & 0.8074 & 0.9254 & 0.9732 & 0.9764 & 0.972 & 0.8986 & 0.962 & 0.9865 \\
\hline 17 & 0.7873 & 0.7687 & 0.5303 & 0.7363 & 0.8881 & 0.8052 & 0.7912 & 0.5546 & 0.7471 & 0.8911 & 0.8973 & 0.8895 & 0.7447 & 0.8644 & 0.944 \\
\hline 18 & 0.9535 & 0.9327 & 0.7595 & 0.9049 & 0.986 & 0.9593 & 0.9435 & 0.7786 & 0.9143 & 0.9868 & 0.9794 & 0.9713 & 0.8824 & 0.9562 & 0.9934 \\
\hline 19 & 0.9393 & 0.9186 & - & 0.8895 & 0.9706 & 0.9451 & 0.9272 & - & 0.8966 & 0.9715 & 0.9722 & 0.9629 & - & 0.9469 & 0.9856 \\
\hline 20 & 0.8866 & 0.8595 & - & 0.8453 & 0.9494 & 0.8975 & 0.8743 & - & 0.8555 & 0.9511 & 0.9474 & 0.935 & - & 0.9249 & 0.9752 \\
\hline
\end{tabular}

\begin{tabular}{|c|c|c|c|c|c|c|c|c|c|c|c|c|c|c|c|}
\hline & 92 & 6966 & 0.4691 & \begin{tabular}{l|l}
0.6723 \\
\end{tabular} & 8382 & 0.7687 & 301 & 0.5015 & 0.69 & 0.8458 & 0.8768 & 09545 & 082 & 07 & \\
\hline$a x$ & & 80 & 0. & 0.9239 & 986 & 93 & 0.9458 & 8 & 1 & & 94 & & & & \\
\hline & 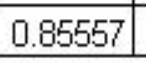 & & & & & & & & & & & & & & \\
\hline
\end{tabular}




\begin{tabular}{|c|c|c|c|c|c|c|c|c|c|c|c|c|c|c|c|}
\hline \multirow{4}{*}{ Network } & \multicolumn{15}{|c|}{ Calibration } \\
\hline & \multicolumn{5}{|c|}{ R squared } & \multicolumn{5}{|c|}{ r squared } & \multicolumn{5}{|c|}{ Correlation coefficient } \\
\hline & & & & & & & & & & & & & & & \\
\hline & C1 & $\mathrm{C} 2$ & $\mathrm{C} 3$ & $\mathrm{CH}$ & $\mathrm{C5}$ & C1 & $\mathrm{C} 2$ & $\mathrm{CB}$ & $\mathrm{CH}$ & $\mathrm{C5}$ & C1 & $\mathrm{C} 2$ & $\mathrm{C3}$ & $\mathrm{CA}$ & $\mathrm{C5}$ \\
\hline 1 & 0.4394 & 0.5234 & 0.2853 & 0.518 & 0.7061 & 0.4698 & 0.5297 & 0.2976 & 0.5272 & 0.7181 & 0.6854 & 0.7278 & 0.5455 & 0.7261 & 0.8474 \\
\hline 2 & 0.4677 & 0.553 & 0.298 & 0.5918 & & 0.4712 & 0.5555 & 0.2989 & 0.5929 & 0.7645 & 0.6864 & 0.7453 & 0.5467 & 0.77 & 0.8744 \\
\hline 3 & 0.5843 & 0.5749 & 0.3079 & 0.6028 & 0.7712 & 0.5845 & 0.5765 & 0.3086 & 0.603 & 0.7715 & 0.7465 & 0.7593 & 0.5555 & 0.7765 & 0.8784 \\
\hline 4 & 0.6847 & 0.6435 & 0.3822 & 0.6278 & 0.7862 & 0.6851 & 0.6435 & 0.3831 & 0.6286 & & 0.8277 & 0.8022 & 0.619 & 0.7928 & 0.8881 \\
\hline 5 & 0.6998 & 0.6259 & 0.413 & 0.6118 & 0.77 & 0.7003 & 0.6259 & 0.4138 & 0.6131 & 0.7733 & 0.8368 & 0.7911 & 0.6433 & 0.783 & 0.8794 \\
\hline 6 & 0.7458 & 0.6607 & 0.4299 & 0.6104 & 0.7955 & 0.7524 & 0.6644 & 0.435 & 0.6119 & 0.797 & 0.8674 & 0.8151 & 0.6596 & 0.7823 & 0.8927 \\
\hline 7 & 0.779 & 0.733 & 0.4684 & 0.6587 & 0.8247 & 0.7835 & 0.7377 & 0.4719 & 0.659 & 0.8257 & 0.8852 & 0.8589 & 0.6869 & 0.8118 & 0.9087 \\
\hline 8 & 0.7949 & 0.7493 & 0.4859 & 0.6827 & 0.8418 & 0.808 & 0.7646 & 0.4982 & 0.6884 & 0.8435 & 0.8989 & 0.8744 & 0.7058 & 0.8297 & 0.9184 \\
\hline 9 & 0.8316 & 0.7698 & 0.4535 & 0.6945 & 0.878 & 0.8417 & 0.7781 & 0.4555 & 0.6996 & 0.8819 & 0.9175 & 0.8821 & 0.6749 & 0.8364 & 0.9391 \\
\hline 10 & 0.8291 & 0.7527 & 0.4927 & 0.7127 & 0.8771 & 0.8326 & 0.7568 & 0.4928 & 0.7181 & 0.881 & 0.9125 & 0.8699 & 0.702 & 0.8474 & 0.9386 \\
\hline 11 & 0.6474 & 0.6115 & 0.3376 & 0.5268 & 0.7154 & 0.6485 & 0.6116 & 0.3489 & 0.5311 & 0.7171 & 0.8053 & 0.782 & 0.5906 & 0.7288 & 0.8468 \\
\hline 12 & 0.816 & 0.7414 & 0.5669 & 0.763 & 0.91 & 0.8176 & 0.742 & 0.5686 & 0.763 & 0.9101 & 0.9042 & 0.8614 & 0.754 & 0.8735 & 0.954 \\
\hline 13 & 0.7836 & 0.7078 & 0.5334 & 0.7237 & 0.8755 & 0.7863 & 0.7092 & 0.5372 & 0.7247 & 0.8777 & 0.8868 & 0.8421 & 0.7329 & 0.8513 & 0.9369 \\
\hline 14 & 0.7934 & 0.7089 & 0.5496 & 0.739 & 0.8811 & 0.7938 & 0.71 & 0.5505 & 0.7394 & 0.8815 & 0.8909 & 0.8426 & 0.742 & 0.8599 & 0.9389 \\
\hline 15 & 0.7928 & 0.708 & 0.5397 & 0.727 & 0.873 & 0.7929 & 0.7091 & 0.5403 & 0.7278 & 0.8737 & 0.8905 & 0.8421 & 0.735 & 0.8531 & 0.9347 \\
\hline 16 & 0.7809 & 0.7185 & 0.5321 & 0.7278 & 0.8733 & 0.7817 & 0.7194 & 0.5327 & 0.7279 & 0.8735 & 0.8841 & 0.8482 & 0.7298 & 0.8532 & 0.9346 \\
\hline 17 & 0.5057 & 0.4857 & 0.2782 & 0.4958 & 0.6475 & 0.5057 & 0.4859 & 0.2844 & 0.496 & 0.6475 & 0.7112 & 0.6971 & 0.5333 & 0.7043 & 0.8047 \\
\hline 18 & 0.783 & 0.7156 & 0.4468 & 0.7145 & 0.8741 & 0.7842 & 0.716 & 0.4503 & 0.7152 & 0.875 & 0.8856 & 0.8462 & 0.671 & 0.8457 & 0.9354 \\
\hline 19 & 0.8353 & 0.7387 & - & 0.7406 & 0.9028 & 0.8361 & 0.7391 & - & 0.7413 & 0.9036 & 0.9144 & 0.8597 & - & 0.861 & 0.9506 \\
\hline 20 & 0.8035 & 0.7511 & - & 0.7526 & 0.9202 & 0.8084 & 0.7547 & - & 0.753 & 0.9203 & 0.8991 & 0.8687 & - & 0.8678 & 0.9593 \\
\hline
\end{tabular}

\begin{tabular}{|c|c|c|c|c|c|c|c|c|c|c|c|c|c|c|c|}
\hline in & 394 & 0.4857 & \begin{tabular}{l|l|}
0.2782 \\
\end{tabular} & 0.4958 & 0.6475 & 0.4698 & \begin{tabular}{l|l|}
0.4859 \\
\end{tabular} & 0.2844 & 0.496 & 0.6475 & 0.6854 & 0.6971 & 0.5333 & 0.7043 & \\
\hline$a x$ & & 0. & 69 & 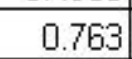 & 02 & 7 & 0 & 86 & 0.763 & 003 & 0.9175 & 91 & & & \\
\hline & & 067 & 0.43339 & 06611 & 082435 & 072422 & 067 & 13713 & $6 E^{-}-2$ & 082627 & ก84682 | & & 6571 & & \\
\hline
\end{tabular}




\begin{tabular}{|c|c|c|c|c|c|c|c|c|c|c|c|c|c|c|c|}
\hline \multirow{4}{*}{ Network } & \multicolumn{15}{|c|}{ Verification } \\
\hline & \multicolumn{5}{|c|}{ R squared } & \multicolumn{5}{|c|}{ r squared } & \multicolumn{5}{|c|}{ Correlation coefficient } \\
\hline & & & & & & & & & & & & & & & \\
\hline & C1 & C2 & $\mathrm{C} 3$ & $\mathrm{CA}$ & $\mathrm{C} 5$ & C1 & $\mathrm{C} 2$ & $\mathrm{C} 3$ & $\mathrm{CH}$ & $\mathrm{C} 5$ & C1 & $\mathrm{C} 2$ & $\mathrm{C} 3$ & $\mathrm{CH}$ & $\mathrm{C5}$ \\
\hline 1 & 0.413 & 0.4649 & 0.2468 & 0.4975 & 0.667 & 0.4407 & 0.4747 & 0.2667 & 0.5098 & 0.6819 & 0.6639 & 0.689 & 0.5164 & 0.714 & 0.8258 \\
\hline 2 & 0.3473 & 0.4169 & 0.2287 & 0.5069 & 0.6841 & 0.3565 & 0.417 & 0.2304 & 0.5075 & 0.6877 & 0.5971 & 0.6458 & 0.48 & 0.7124 & 0.8293 \\
\hline 3 & 0.4117 & 0.5329 & 0.2597 & 0.5841 & 0.7568 & 0.4269 & 0.5329 & 0.2629 & 0.5842 & 0.7586 & 0.6534 & 0.73 & 0.5127 & 0.7644 & 0.871 \\
\hline 4 & 0.5438 & 0.6409 & 0.317 & 0.6155 & 0.7833 & 0.5515 & 0.6415 & \begin{tabular}{|l|}
0.3202 \\
\end{tabular} & 0.6171 & 0.7883 & 0.7426 & 0.8009 & 0.5659 & 0.7856 & 0.8878 \\
\hline 5 & 0.5828 & 0.534 & 0.3108 & 0.5785 & 0.726 & 0.5888 & 0.5366 & 0.3157 & 0.5803 & 0.7308 & 0.7673 & 0.7325 & 0.5619 & 0.7618 & 0.8549 \\
\hline 6 & 0.6471 & 0.6225 & 0.3901 & 0.5897 & 0.7638 & 0.6473 & 0.6236 & 0.392 & 0.592 & 0.7674 & 0.8046 & 0.7897 & 0.6261 & 0.7694 & 0.876 \\
\hline 7 & 0.6954 & 0.5817 & 0.4288 & 0.522 & 0.7086 & 0.6959 & 0.5829 & 0.4294 & 0.5304 & 0.7192 & 0.8342 & 0.7635 & 0.6553 & 0.7283 & 0.8481 \\
\hline 8 & 0.7189 & 0.6705 & 0.4109 & 0.6487 & 0.8218 & 0.7208 & 0.6744 & 0.4126 & 0.6504 & 0.8229 & 0.849 & 0.8212 & 0.6423 & 0.8065 & 0.9071 \\
\hline 9 & 0.8058 & 0.7611 & 0.5002 & 0.6701 & 0.8198 & 0.8084 & 0.7644 & 0.504 & 0.6707 & 0.822 & 0.8991 & 0.8743 & 0.7099 & 0.819 & 0.9067 \\
\hline 10 & 0.8043 & 0.728 & 0.468 & 0.6616 & 0.8562 & 0.8058 & 0.8298 & 0.4696 & 0.6631 & 0.8572 & 0.8977 & 0.8543 & 0.6853 & 0.8143 & 0.9259 \\
\hline 11 & 0.829 & 0.8051 & 0.4977 & 0.7566 & 0.9101 & 0.8364 & 0.8149 & 0.498 & 0.7624 & 0.9133 & 0.9145 & 0.9027 & 0.7057 & 0.8732 & 0.9557 \\
\hline 12 & 0.8113 & 0.7509 & 0.5028 & 0.7424 & 0.8631 & 0.8145 & 0.7539 & 0.5061 & 0.7433 & \begin{tabular}{|l}
0.8632 \\
\end{tabular} & 0.9025 & 0.8683 & 0.7114 & 0.8622 & 0.9291 \\
\hline 13 & 0.7327 & 0.7123 & 0.4478 & 0.7184 & 0.8337 & 0.734 & 0.7193 & 0.4515 & 0.7214 & 0.8342 & 0.8567 & 0.8481 & 0.6719 & 0.8493 & 0.9133 \\
\hline 14 & 0.7694 & 0.738 & 0.4614 & 0.7321 & 0.8278 & 0.7694 & 0.7408 & 0.4263 & 0.7322 & 0.828 & 0.8772 & 0.8607 & 0.6799 & 0.8557 & 0.91 \\
\hline 15 & 0.7651 & 0.7315 & 0.4555 & 0.7389 & 0.8302 & 0.7653 & 0.7344 & 0.4564 & 0.7389 & 0.8302 & 0.8748 & \begin{tabular}{|l|}
0.857 \\
\end{tabular} & 0.6755 & 0.8596 & 0.9111 \\
\hline 16 & 0.7606 & 0.7082 & 0.4674 & 0.7348 & 0.8258 & 0.762 & 0.7122 & 0.4681 & 0.7355 & 0.8265 & 0.873 & 0.8439 & 0.6842 & 0.8576 & 0.9091 \\
\hline 17 & 0.5769 & 0.5798 & 0.3638 & 0.6252 & 0.7821 & 0.5771 & 0.5825 & 0.3646 & 0.6261 & 0.7848 & 0.7596 & 0.7632 & 0.6039 & 0.7912 & 0.8859 \\
\hline 18 & 0.7872 & 0.6733 & 0.5261 & 0.6961 & 0.8262 & 0.7891 & 0.6746 & 0.527 & 0.7006 & 0.8315 & 0.8883 & 0.8214 & 0.7259 & 0.837 & 0.9119 \\
\hline 19 & 0.7977 & 0.746 & - & 0.7651 & 0.877 & 0.7983 & 0.7475 & - & 0.767 & 0.8772 & 0.8935 & 0.8646 & - & 0.8758 & 0.9366 \\
\hline 20 & 0.7872 & 0.741 & - & 0.7197 & 0.8524 & 0.7907 & 0.7449 & - & 0.7219 & 0.8527 & 0.8892 & 0.8631 & - & 0.8497 & 0.9234 \\
\hline
\end{tabular}

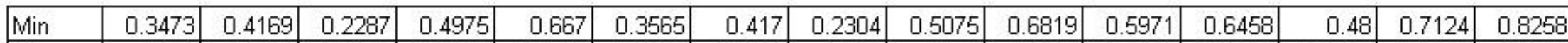
\begin{tabular}{|l|r|r|r|r|r|r|r|r|r|r|r|r|r|r|r|r|r|r|}
\hline Max & 0.829 & 0.8051 & 0.5261 & 0.7651 & 0.9101 & 0.8364 & 0.8298 & 0.527 & 0.767 & 0.9133 & 0.9145 & 0.9027 & 0.7259 & 0.8758 & 0.9557 \\
\hline
\end{tabular} \begin{tabular}{|l|l|l|l|l|l|l|l|l|l|l|l|l|l|l|l|} 
Average & 0.67936 & 0.65698 & 0.40464 & 0.6552 & 0.80079 & 0.68397 & 0.66514 & 0.40564 & 0.65774 & 0.80388 & 0.82191 & 0.80971 & 0.63412 & 0.80935 & 0.89594 \\
\hline
\end{tabular} 


\section{Appendix E The neural network prediction for the crosswell} seismic traces- the verification set
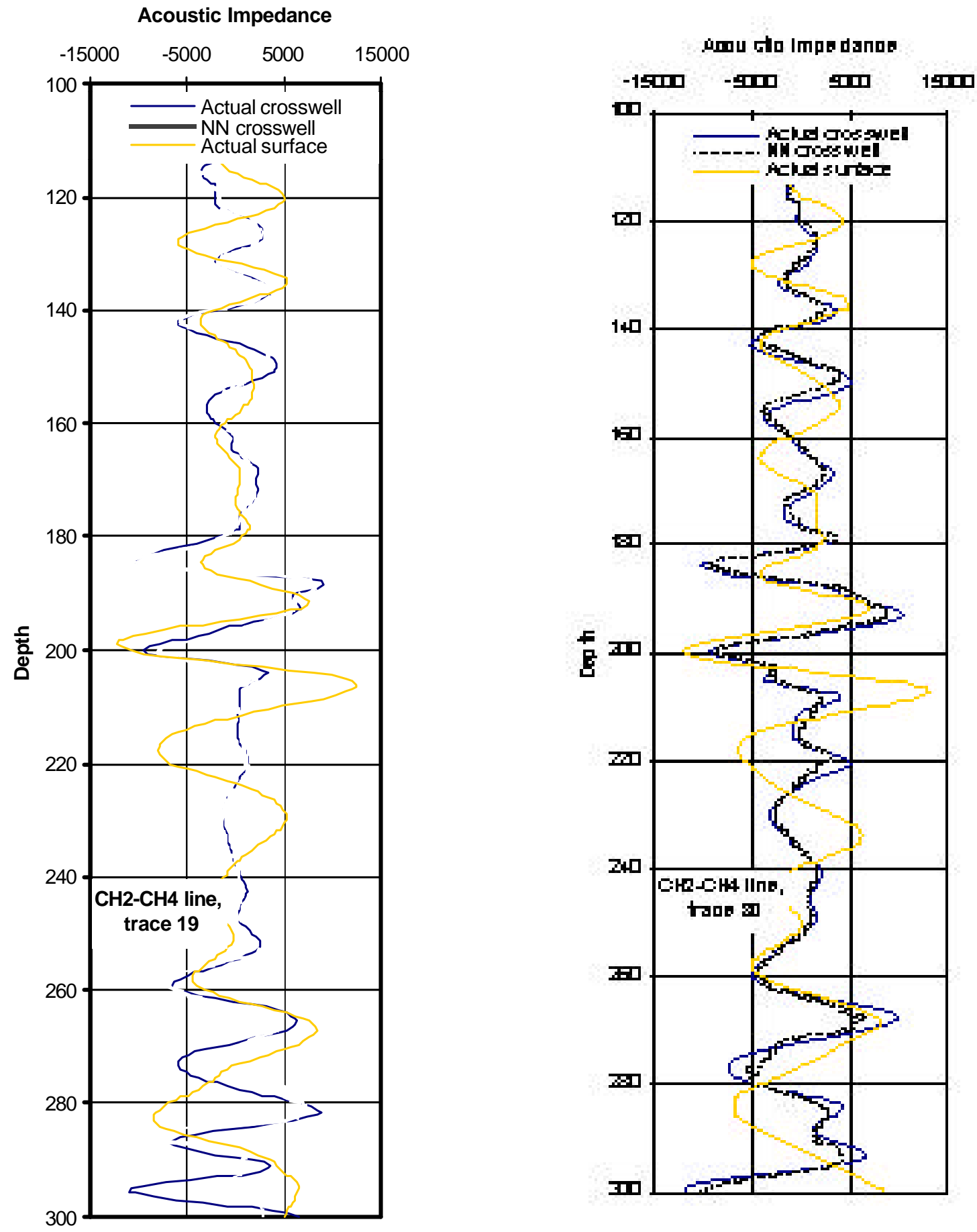

Figure E1. Predicted and actual acoustic impedance for trace 19, $\mathrm{CH} 2-\mathrm{CH} 4$
Figure E2. Predicted and actual acoustic impedance for trace 30, $\mathrm{CH} 2-\mathrm{CH} 4$ 


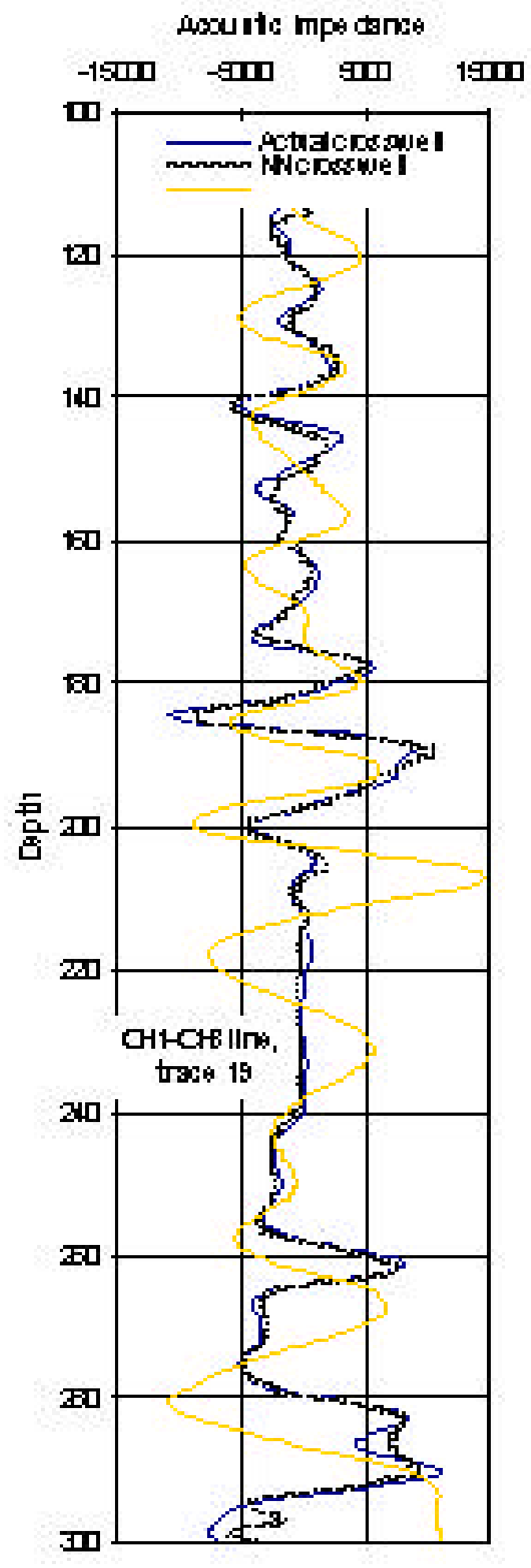

Figure E3. Predicted and actual acoustic impedance for trace 19, $\mathrm{CH1}-\mathrm{CH} 3$

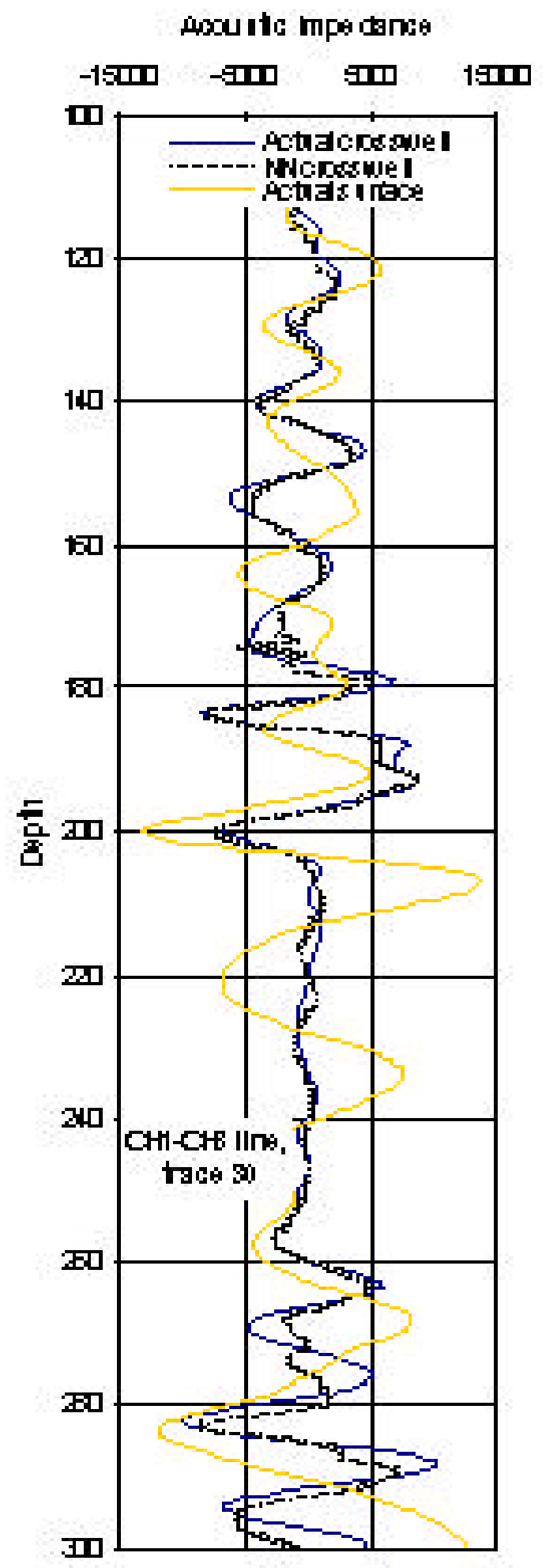



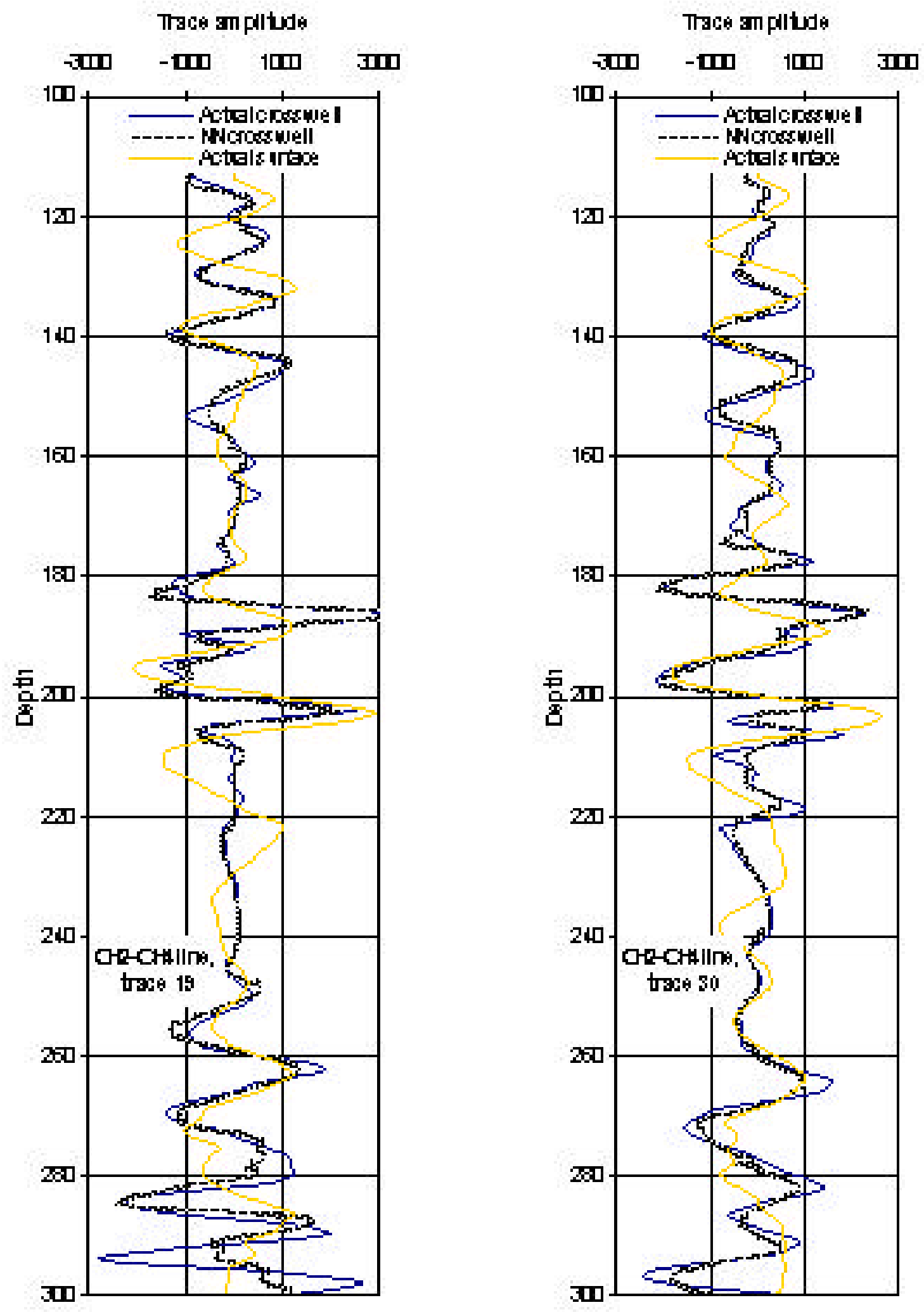

Figure E5. Predicted and actual trace Figure E6. Predicted and actual trace amplitude for trace 19, $\mathrm{CH} 2-\mathrm{CH} 4$ amplitude for trace 30, $\mathrm{CH2}-\mathrm{CH} 4$ 

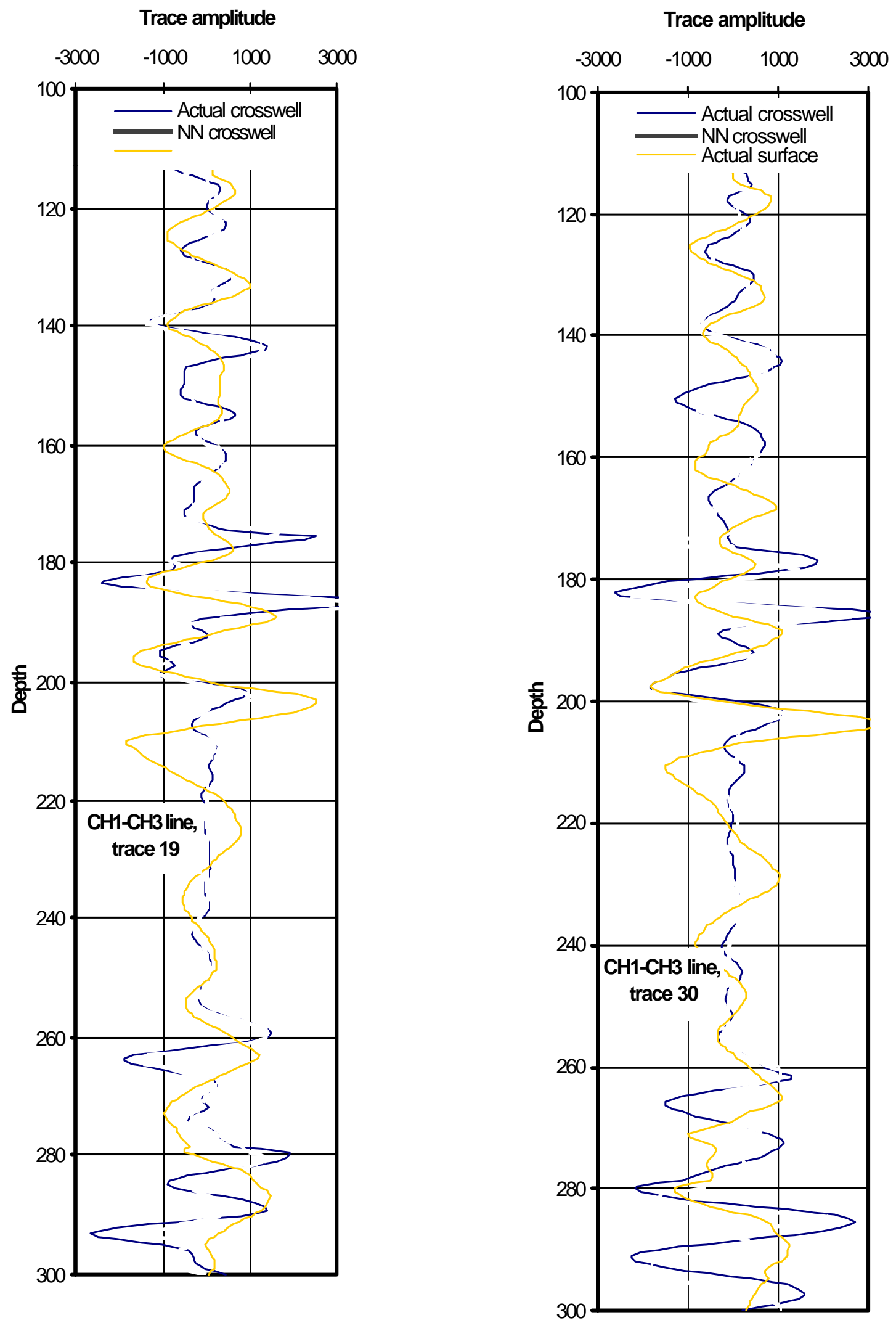

Figure E7. Predicted and actual trace amplitude for trace 19, CH1-CH3

Figure E8. Predicted and actual trace amplitude for trace 30, CH1-CH3 

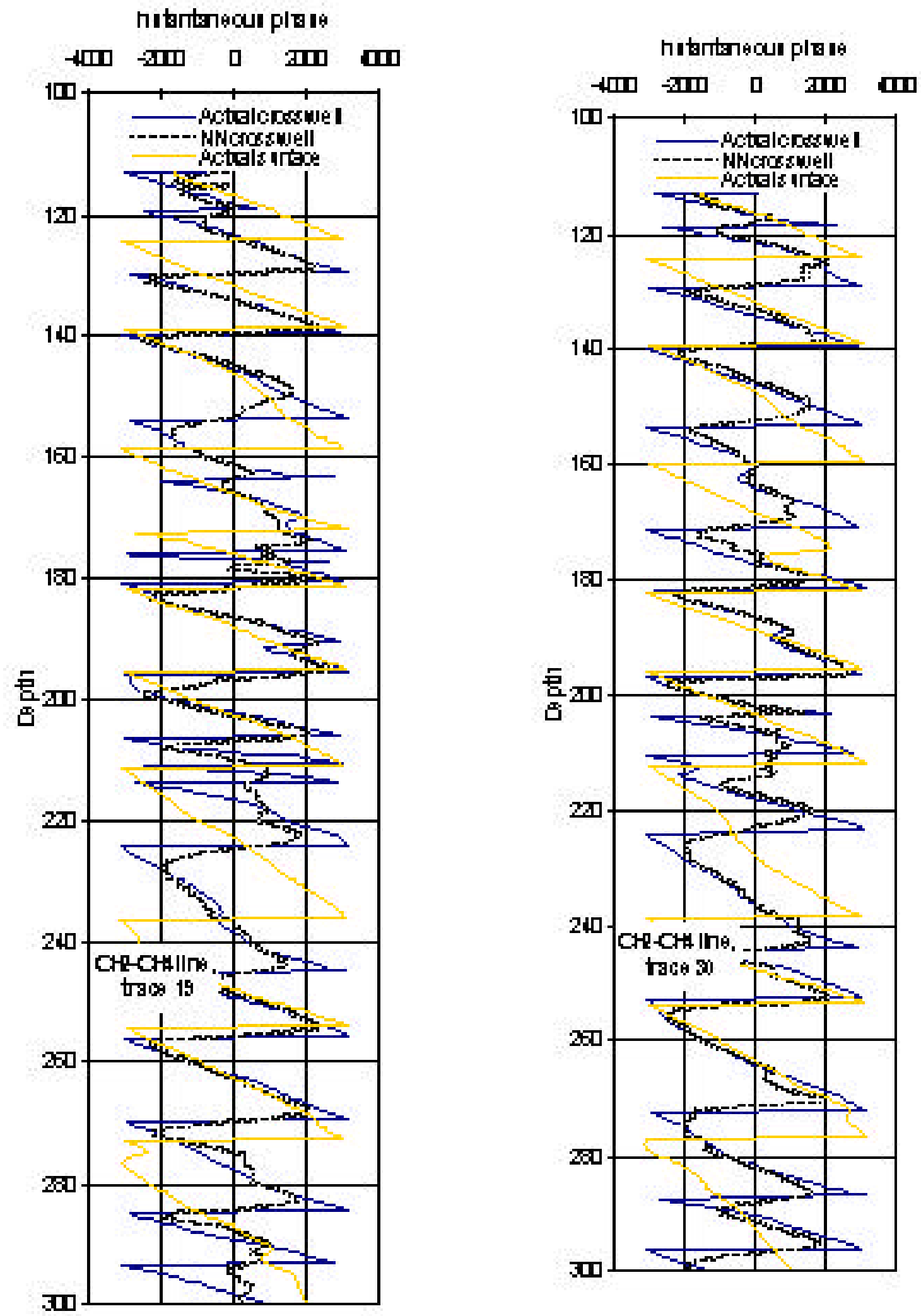

Figure E9. Predicted and actual Figure E10. Predicted and actual instantaneous phase for trace 19, $\mathrm{CH2}-\mathrm{CH} 4$ instantaneous phase for trace 30, $\mathrm{CH} 2-\mathrm{CH} 4$ 

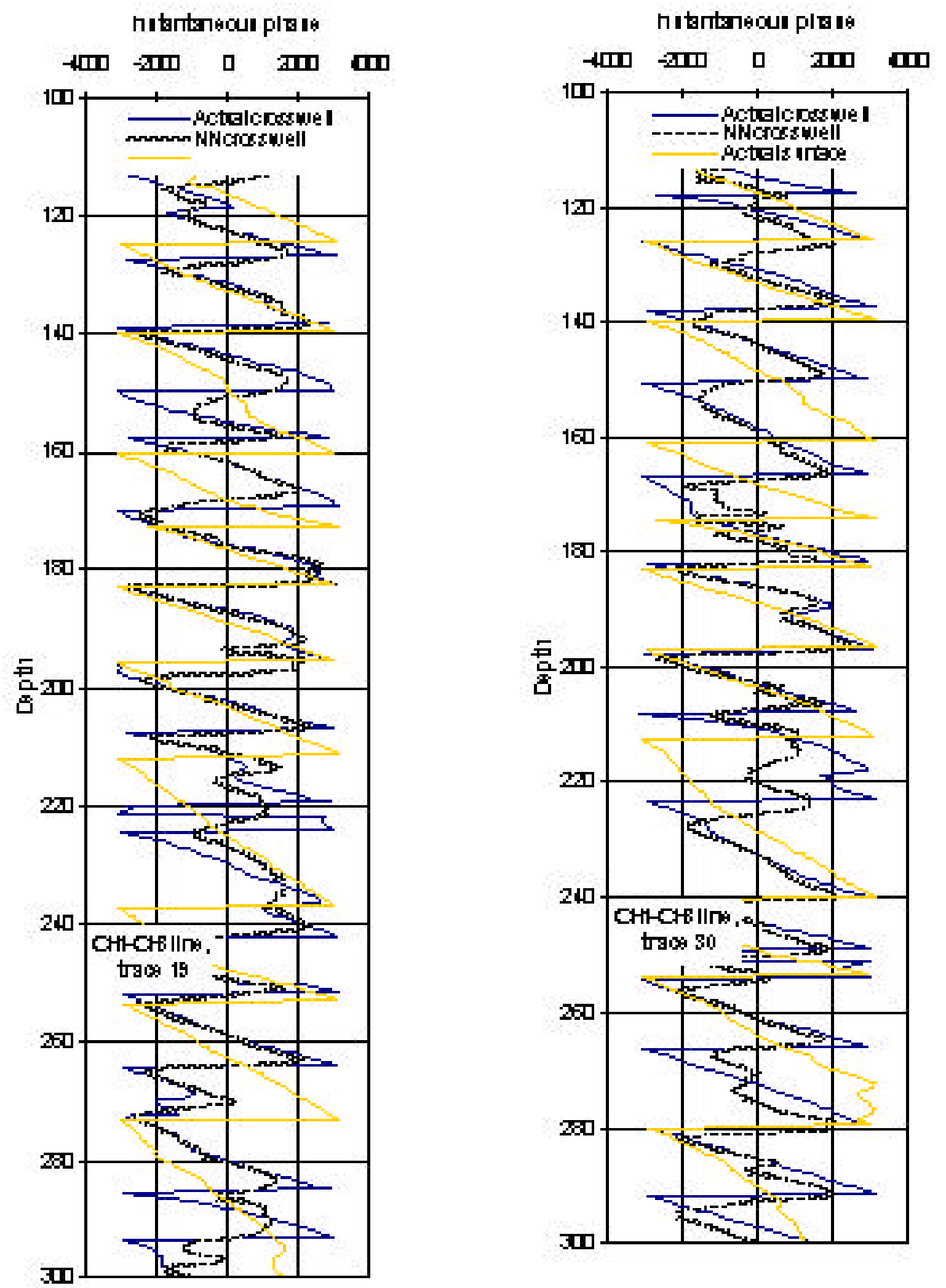

Figure E11. Predicted and actual Figure E12. Predicted and actual instantaneous phase for trace 19, $\mathrm{CH1}-\mathrm{CH}$ instantaneous phase for trace 30, $\mathrm{CH1}-\mathrm{CH}$ 

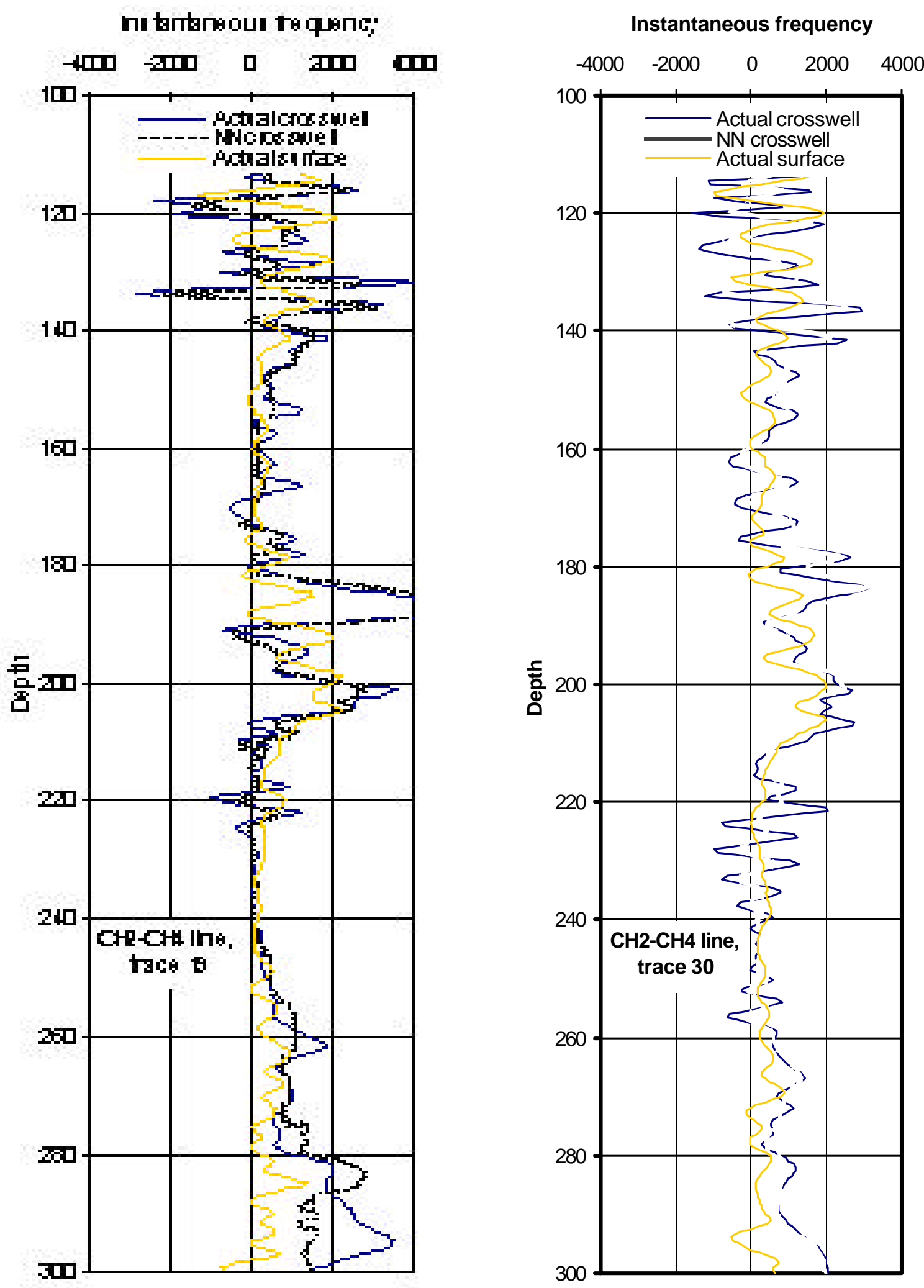

Figure E13. Predicted and actual Figure E14. Predicted and actual instantaneous frequency for trace instantaneous frequency for trace 19, $\mathrm{CH} 2-\mathrm{CH} 4$ 30, $\mathrm{CH} 2-\mathrm{CH} 4$ 

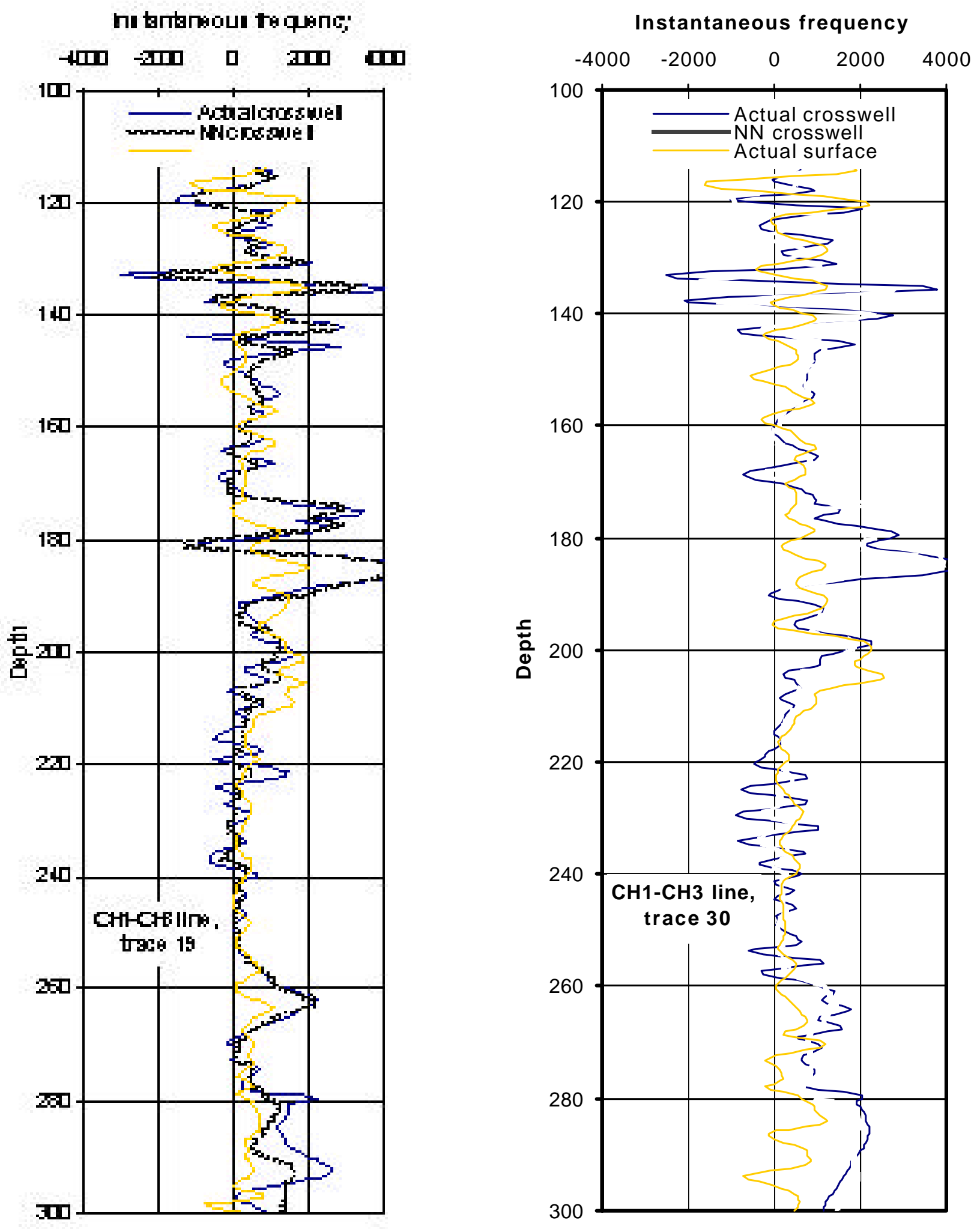

Figure E15. Predicted and actual Figure E16. Predicted and actual instantaneous frequency for trace 19, instantaneous frequency for trace 30, CH1-CH3 CH1-CH3 


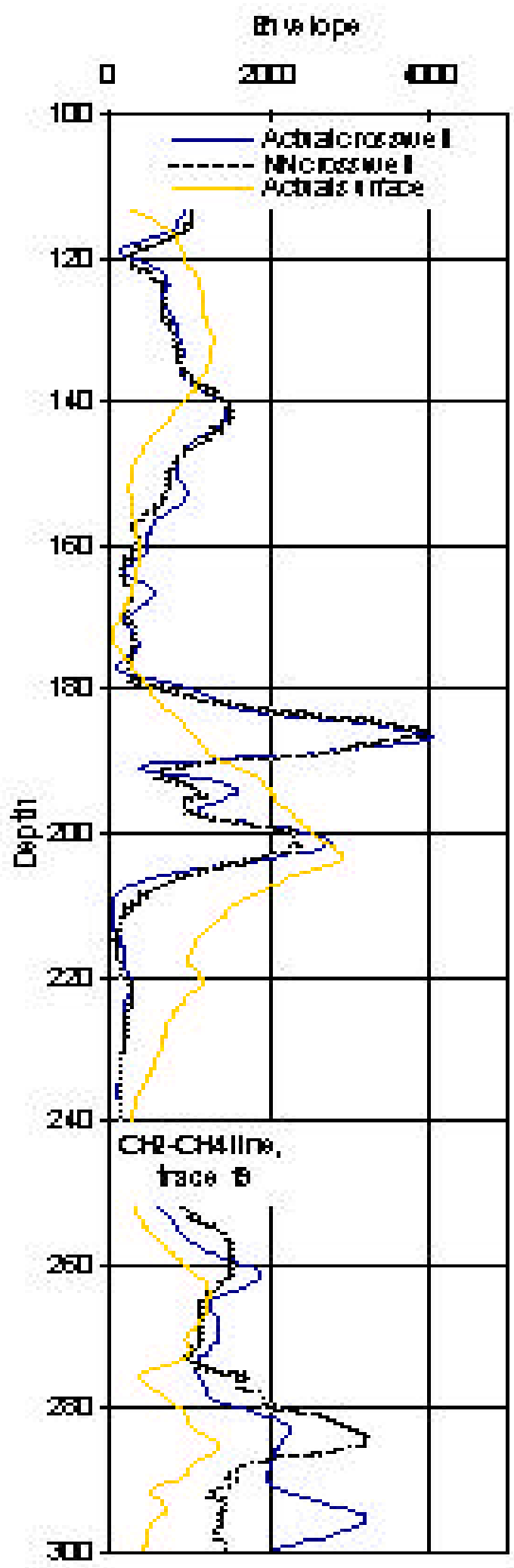

Figure E17. Predicted and actual envelope for trace 19, $\mathrm{CH} 2-\mathrm{CH} 4$

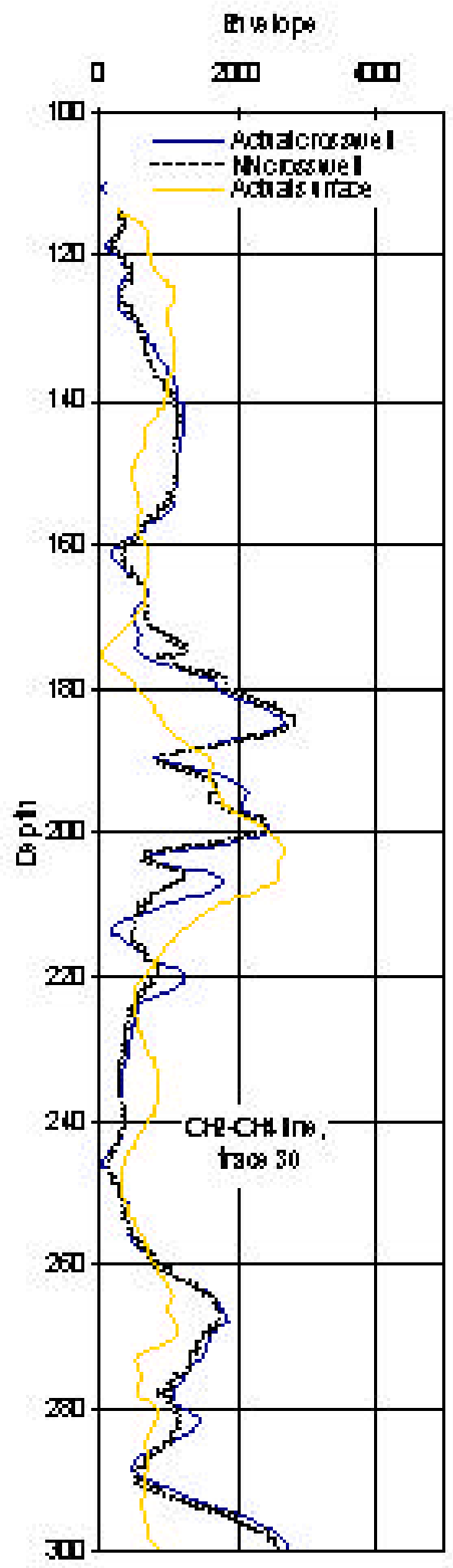

Figure E18. Predicted and actual envelope for trace 30, $\mathrm{CH} 2-\mathrm{CH} 4$ 

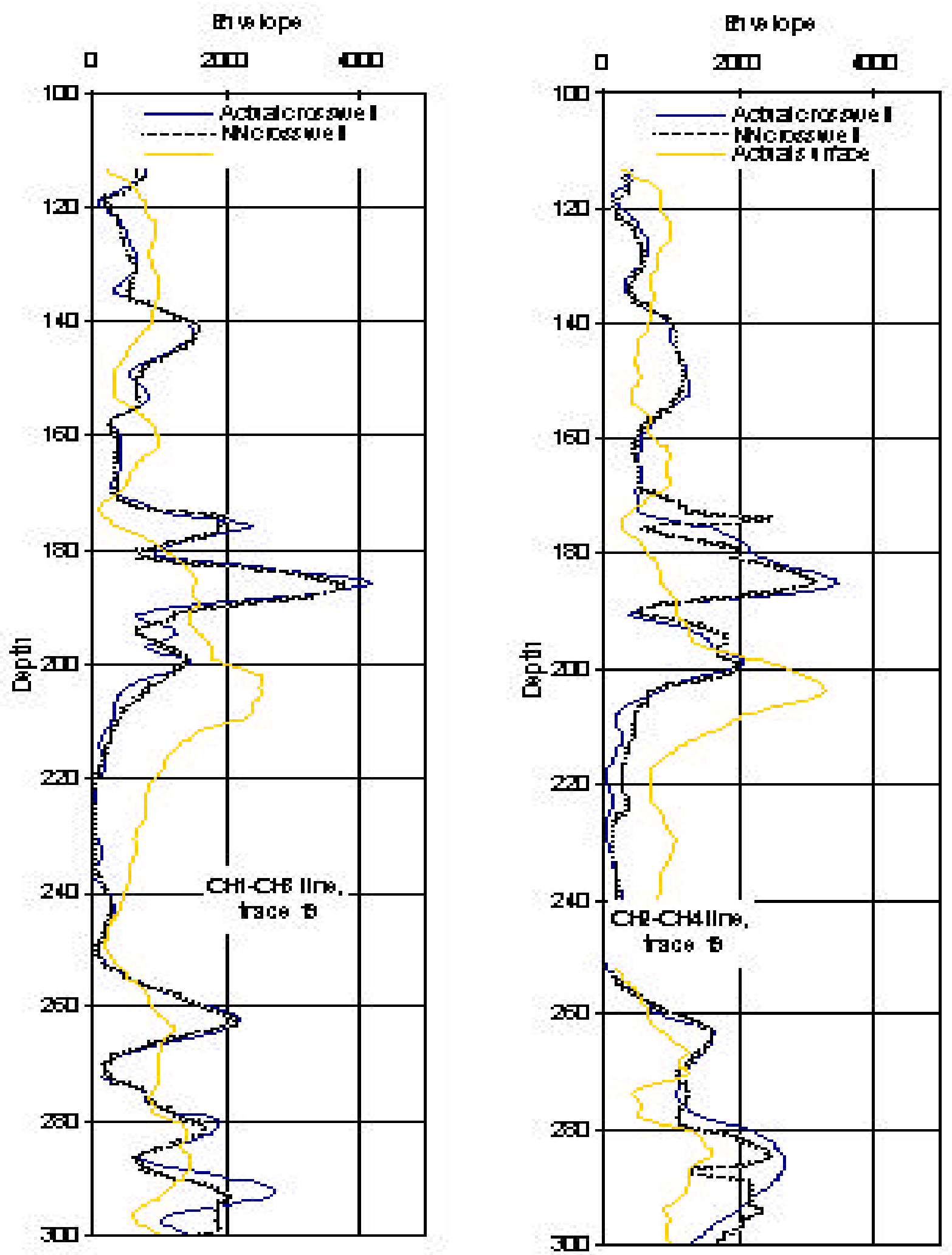

Figure E19. Predicted and actual envelope for trace 19, CH1-CH3

Figure E20. Predicted and actual envelope for trace 30, CH1-CH3 
Appendix F: Crossplots of the seismic attributes: real values vs. predicted values (best network)

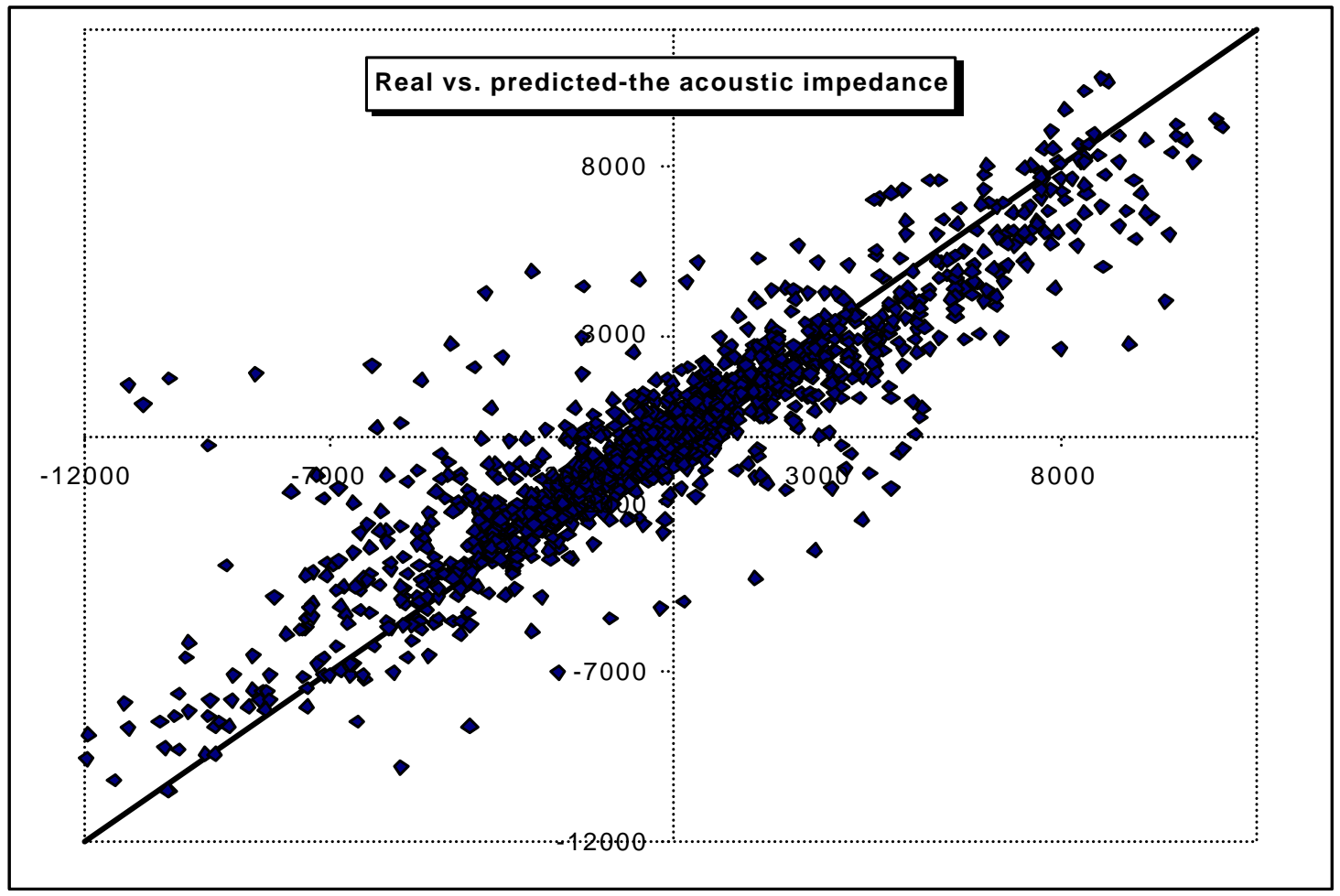

Figure F1. Crossplot between the real and the predicted values for the acoustic impedance

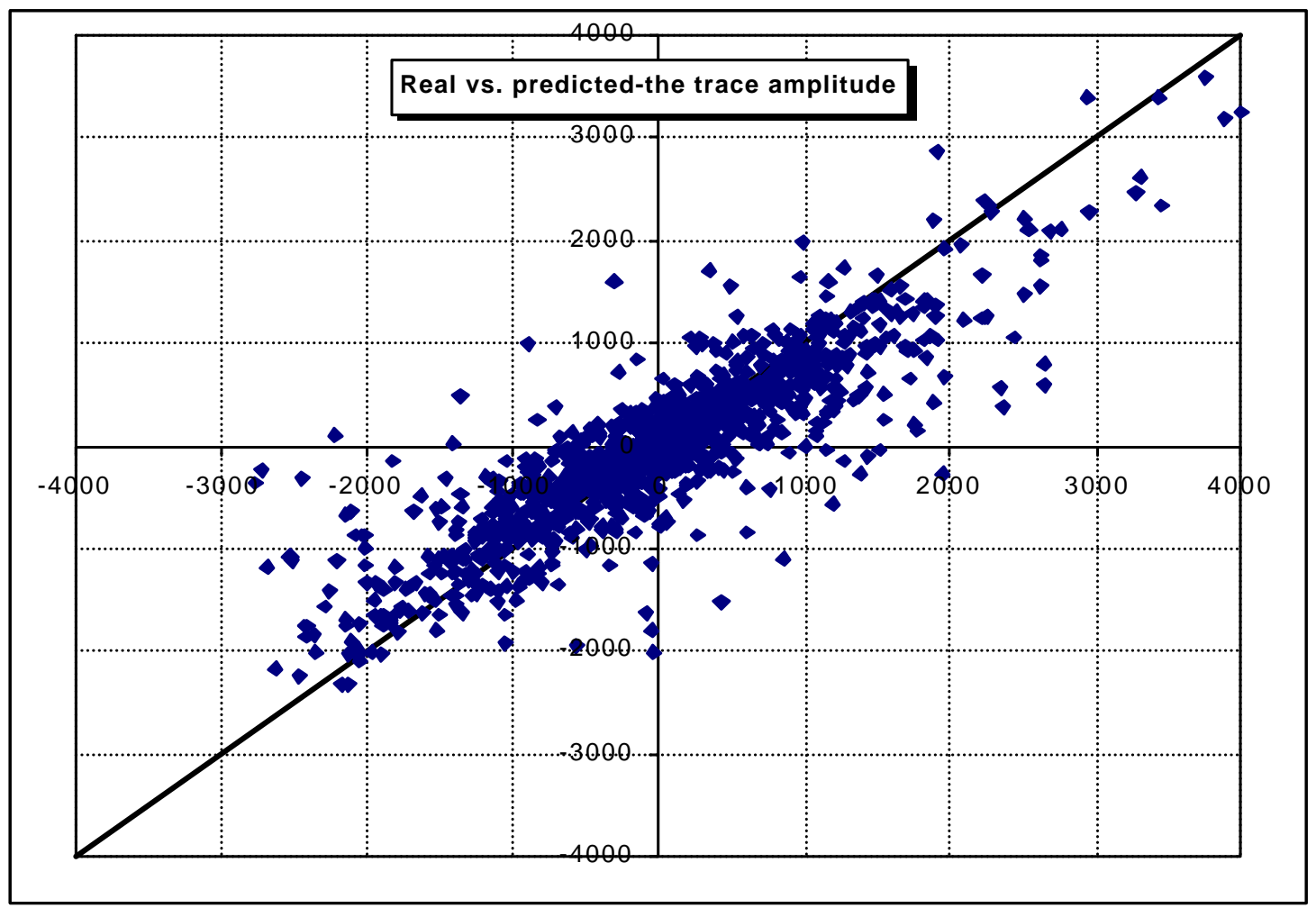

Figure F2. Crossplot between the real and the predicted values for the trace amplitude 


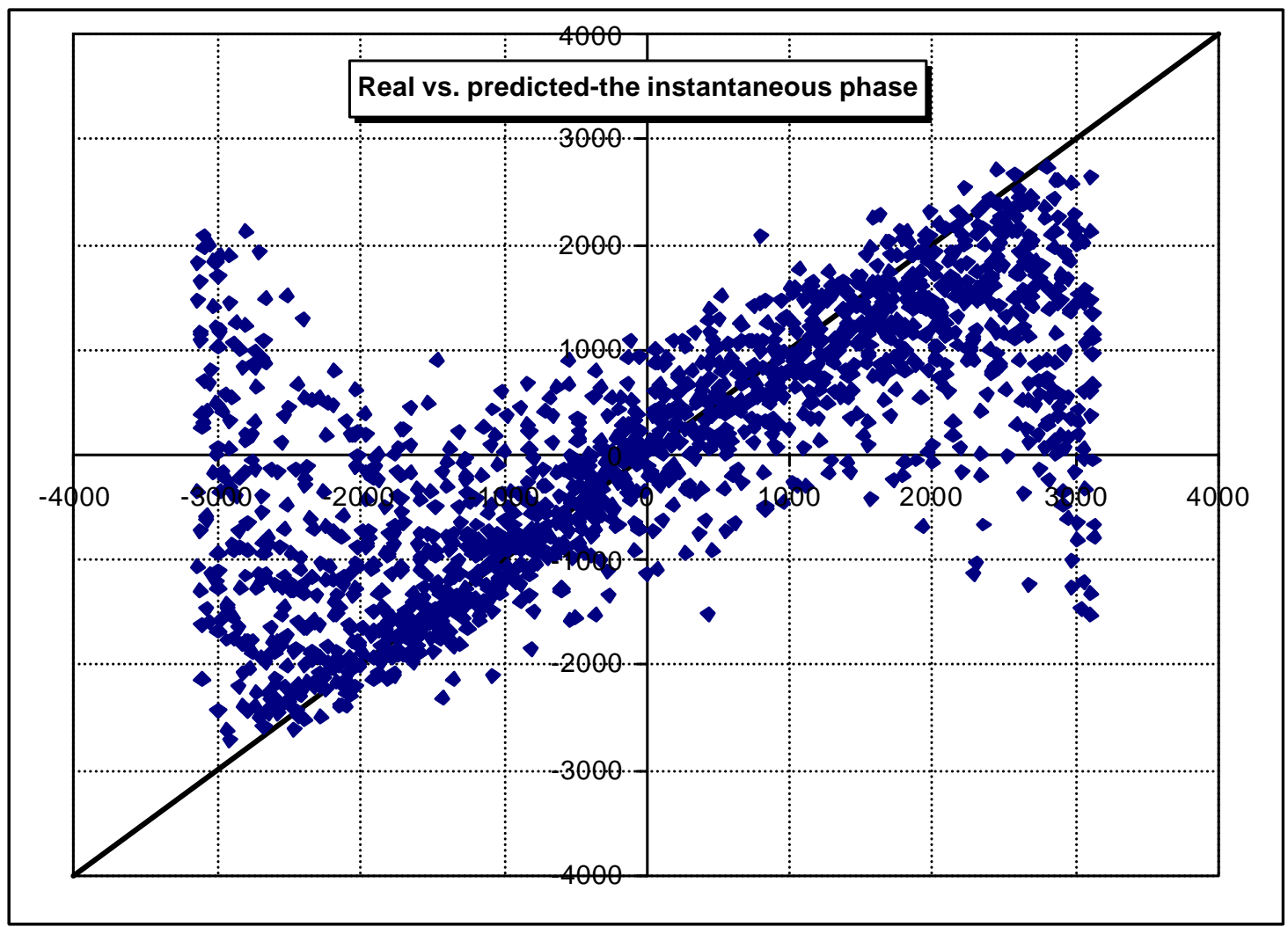

Figure F3. Crossplot between the real and the predicted values for the instantaneous phase

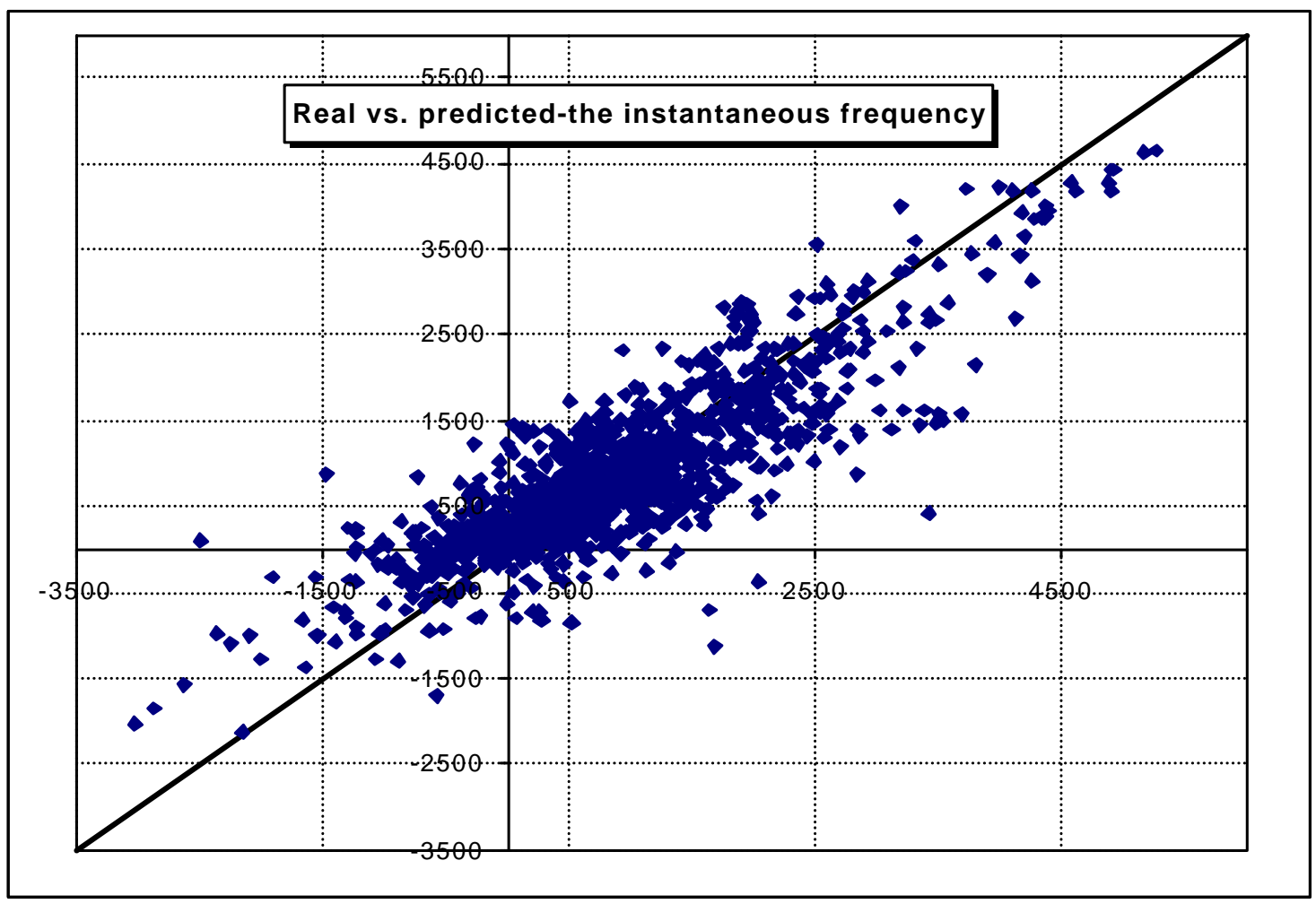

Figure F4. Crossplot between the real and the predicted values for the instantaneous frequency 


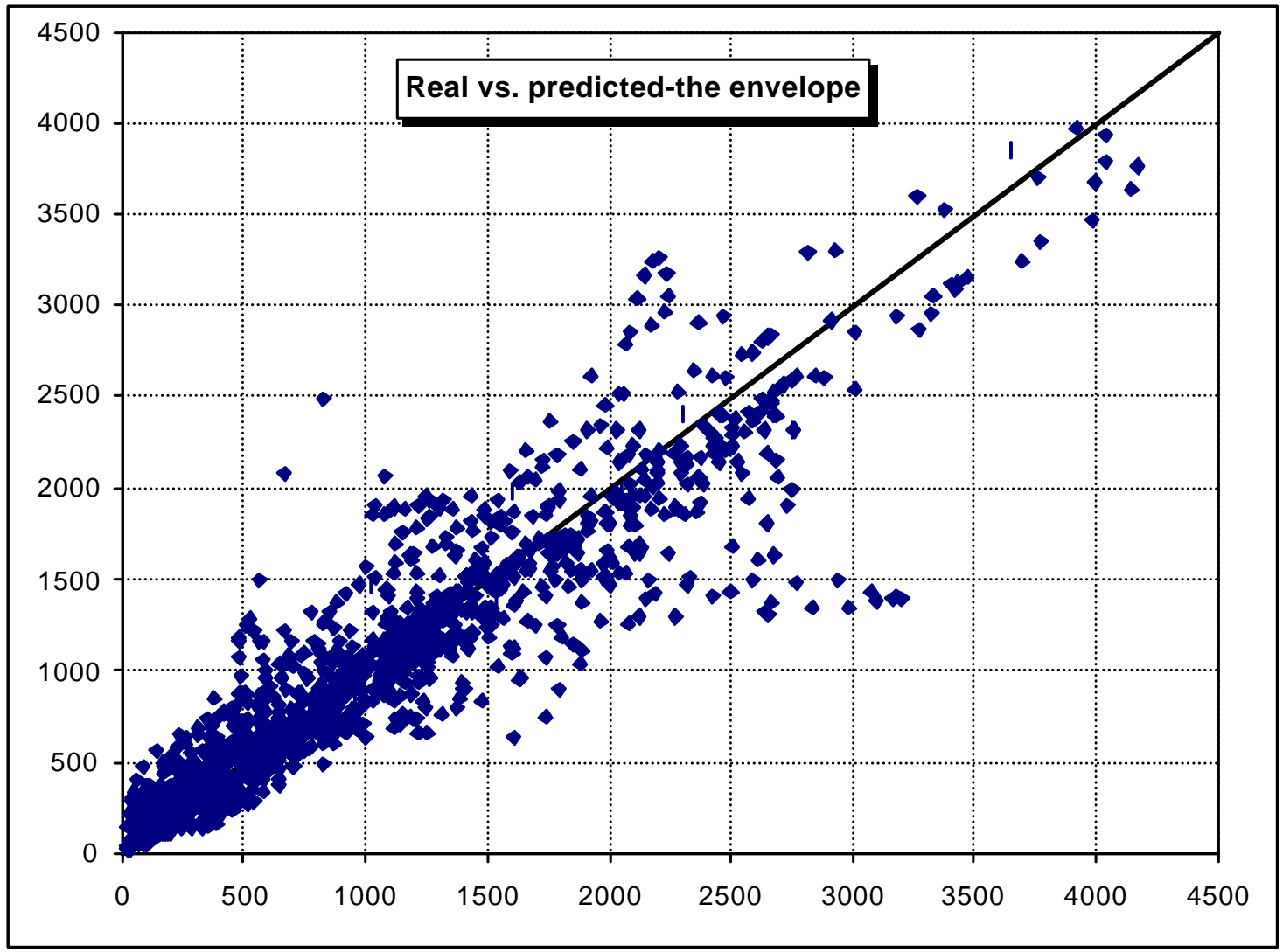

Figure F2. Crossplot between the real and the predicted values for the envelope 